\title{
HYDROLOGIC MONITORING IN THE AREA OF THE TENNESSEE-TOMBIGBEE WATERWAY, MISSISSIPPI-ALABAMA, FISCAL YEAR 1990
}

By Fred Morris III

U.S. GEOLOGICAL SURVEY

Open-File Report 91-521

Prepared in cooperation with the

U.S. ARMY CORPS OF ENGINEERS

MOBILE DISTRICT

Jackson, Mississippi

1991 


\section{U.S. DEPARTMENT OF THE INTERIOR \\ MANUEL LUJAN, JR., Secretary}

\section{U.S. GEOLOGICAL SURVEY}

Dallas L. Peck, Director

For additional information write to:

U.S. Geological Survey

Water Resources Division

$100 \mathrm{~W}$. Capitol Street, Suite 710

Jackson, MS 39269
U.S. Geological Survey

Water Resources Division

520 19th Avenue

Tuscaloosa, AL 35401

Copies of this report can be purchased from:

U.S. Geological Survey

Books and Open-File Reports

Box 25425, Federal Center

Denver, CO 80225 


\section{CONTENTS}

Page

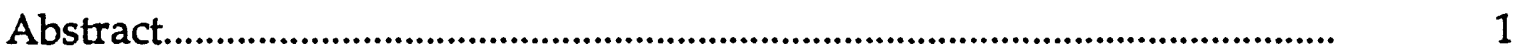

Hydrologic monitoring......................................................................................

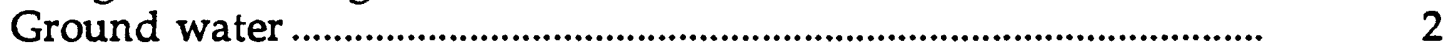

Network ......................................................................................

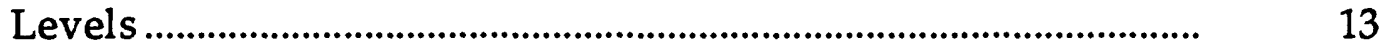

Quality................................................................................................ 13

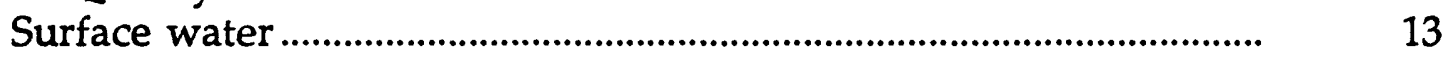

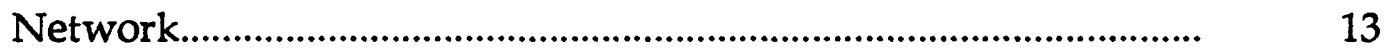

Stage and discharge ............................................................................. 13

Quality.......................................................................................................

Quality assurance............................................................................................. 15

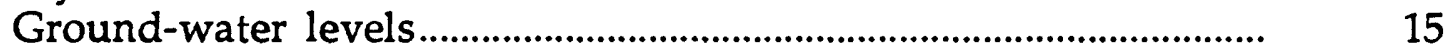

Surface-water stage and discharge............................................................. 15

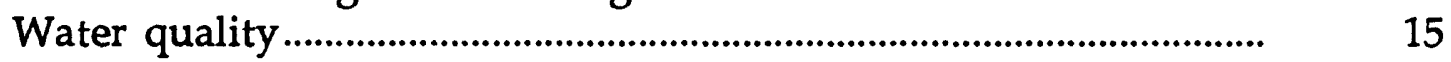

Selected references.....................................................................................

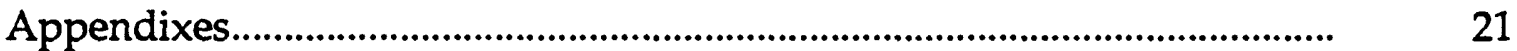

Explanation of codes and abbreviations contained in data tables in the appendixes.

Appendix A--Ground-water data ............................................................. 25

Descriptions of wells.................................................................................... 27

Hydrographs ..........................................................................................

Water-quality analyses ...............................................................................

Appendix B--Surface-water data.................................................................

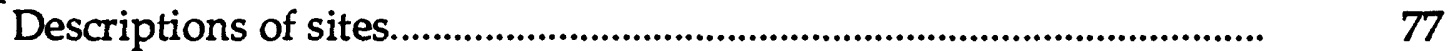

Water-quality analyses .................................................................. 


\section{ILLUSTRATIONS}

Page

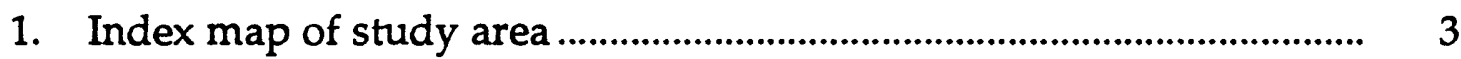

Figures 2-10. Maps showing:

2. Location of hydrologic monitoring sites in the Divide Section........ 4

3. Location of hydrologic monitoring sites in the vicinity of Pools $D$ and $\mathrm{E}$

4. Location of hydrologic monitoring sites in the vicinity of Pool C... 6

5. Location of hydrologic monitoring sites in the vicinity of Pool B... 7

6. Location of hydrologic monitoring sites in the vicinity of Pool A... 8

7. Location of hydrologic monitoring sites in the vicinity of Aberdeen Pool

8. Location of hydrologic monitoring sites in the vicinity of Columbus Pool

9. Location of hydrologic monitoring sites in the vicinity of Aliceville Pool

10. Location of hydrologic monitoring sites in the vicinity of Gainesville Pool. 


\section{CONVERSION FACTORS AND VERTICAL DATUM}

\section{Multiply}

inch (in.)

foot $(\mathrm{ft})$

mile (mi)

square mile $\left(\mathrm{mi}^{2}\right)$

acre-foot (acre-ft)

cubic foot per second $\left(\mathrm{ft}^{3} / \mathrm{s}\right)$

gallon per minute (gal/min)

micromho per centimeter

at $25^{\circ} \mathrm{Celsius}$ (umho/ $\mathrm{cm}$ at $25^{\circ} \mathrm{C}$ )
By $\quad$ To obtain

25.40

0.3048

1.609

2.590

1,233

0.02832

0.06308

1.000 millimeter

meter

kilometer

square kilometer

cubic meter

cubic meter per second

liter per second

microsiemens per centimeter at $25^{\circ}$ Celsius

To convert degrees Celsius $\left({ }^{\circ} \mathrm{C}\right)$ to Fahrenheit $\left({ }^{\circ} \mathrm{F}\right)$, use the following:

$$
{ }^{\circ} \mathrm{F}=9 / 5^{\circ} \mathrm{C}+32
$$

Sea level: In this report "sea level" refers to the National Geodetic Vertical Datum of 1929 (NGVD of 1929)--a geodetic datum derived from a general adjustment of the first-order level nets of both the United States and Canada, formerly called "Sea Level Datum of 1929." 


\title{
HYDROLOGIC MONITORING IN THE AREA OF THE TENNESSEE-TOMBIGBEE WATERWAY, MISSISSIPPI- ALABAMA, FISCAL YEAR 1990
}

\author{
by Fred Morris III
}

\begin{abstract}
This report, the seventeenth and final in a series of annual reports, presents hydrologic data collected in the area of the Tennessee-Tombigbee Waterway during the fiscal year ending September 30, 1990. Included in this report are data on ground-water levels and quality, and surface-water stage, discharge, and quality. These data were obtained at the request of the U.S. Army Corps of Engineers, Mobile District, as part of a comprehensive program to monitor the hydrologic effects of construction and operation of the Waterway.
\end{abstract}




\section{HYDROLOGIC MONITORING}

Surface-water sites and observation wells in the original hydrologic monitoring network, used to define hydrologic conditions in the area of the Tennessee-Tombigbee Waterway prior to construction, are described by Brahana and others (1974) in the U.S. Army Corps of Engineers (COE) report, "First Supplemental Environmental Report, Continuing Environmental Studies, Tennessee-Tombigbee Waterway, Alabama and Mississippi." The present hydrologic monitoring network includes:

- Major aquifers that may have been stressed by the Waterway construction and operation;

- Surface-water sites near locks and dams where the effects of construction may have been greatest, or at sites of inflow or outflow;

- Areas of known or suspected hydrologic problems;

- Selected sites on and near Pickwick Lake and Demopolis Lake.

The purpose of the present hydrologic monitoring network is to document changes in the hydrologic environment that may occur during operation of the Waterway. The locations of all the hydrologic monitoring sites in the vicinity of the Waterway at which data have been collected since the beginning of the project are shown in figures 1 through 10, except for a few sites which are not shown because of the limited coverage of the maps (furnished by the U.S. Army Corps of Engineers, Mobile District).

\section{Ground Water}

\section{Network}

The present ground-water network consists of 134 wells in the regional aquifers and the shallower alluvial and terrace aquifers. The relation between shallow water-bearing units and regional aquifers is described by Brahana and others (1974). The descriptions of wells in the network are tabulated in Appendix A. 


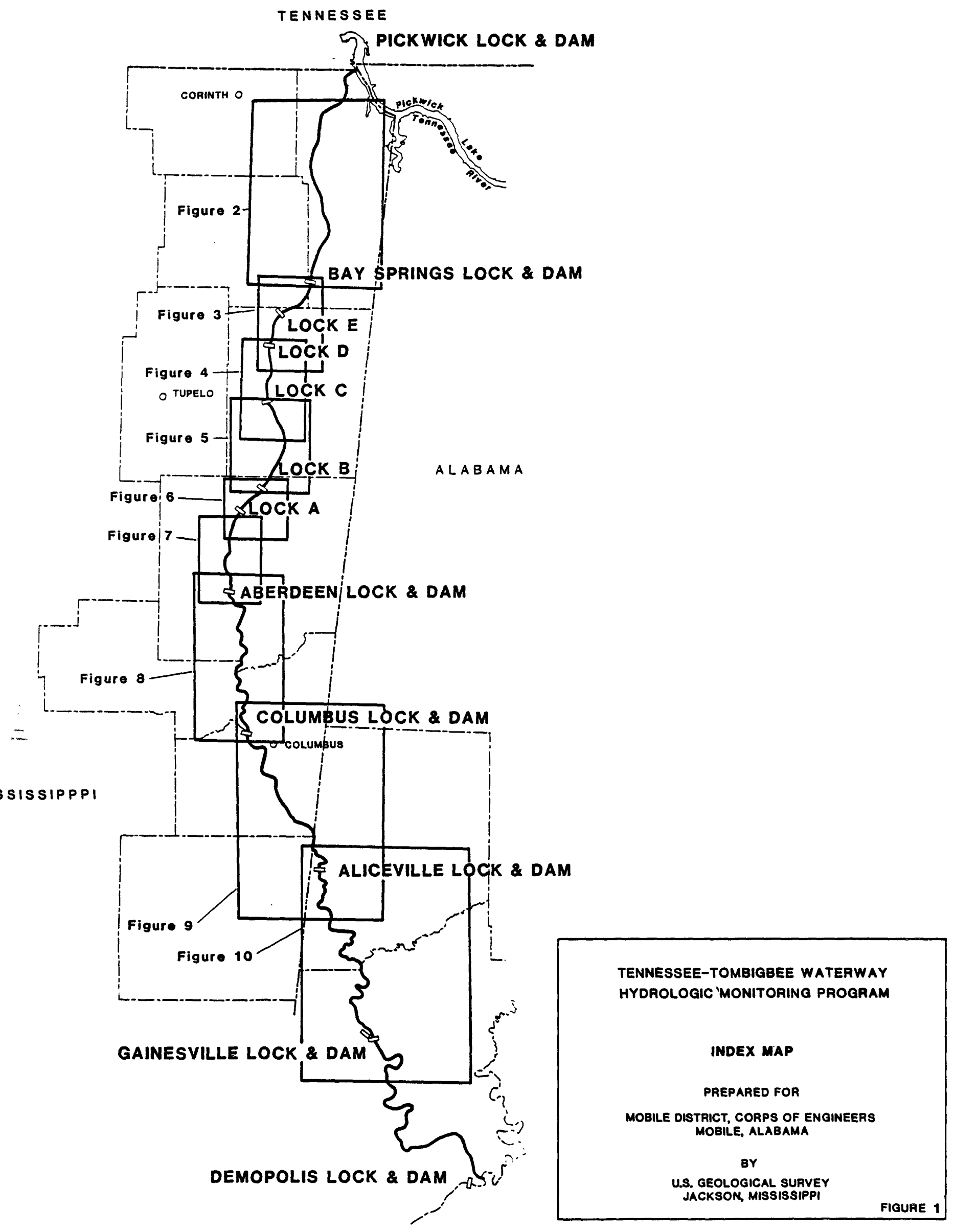




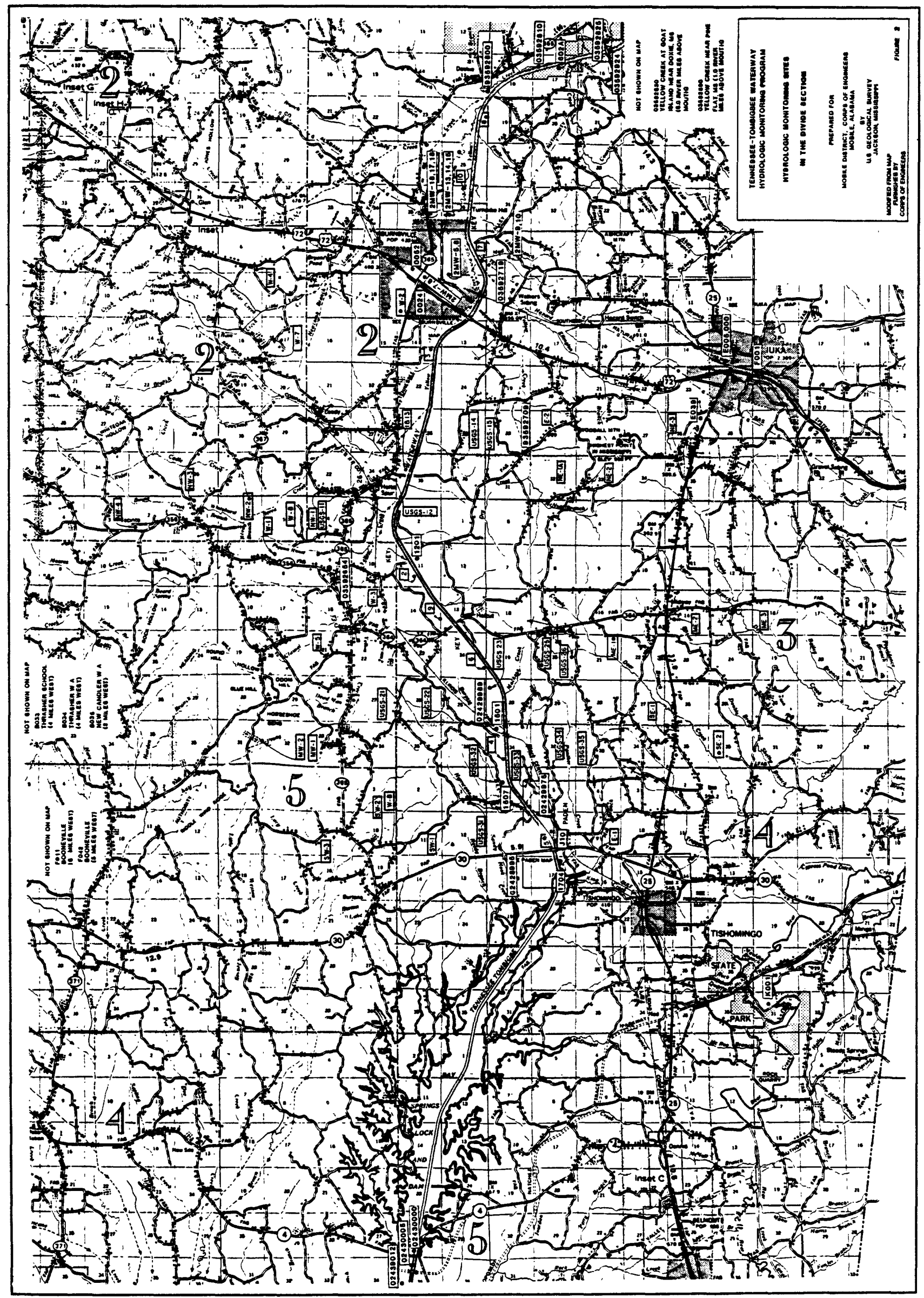




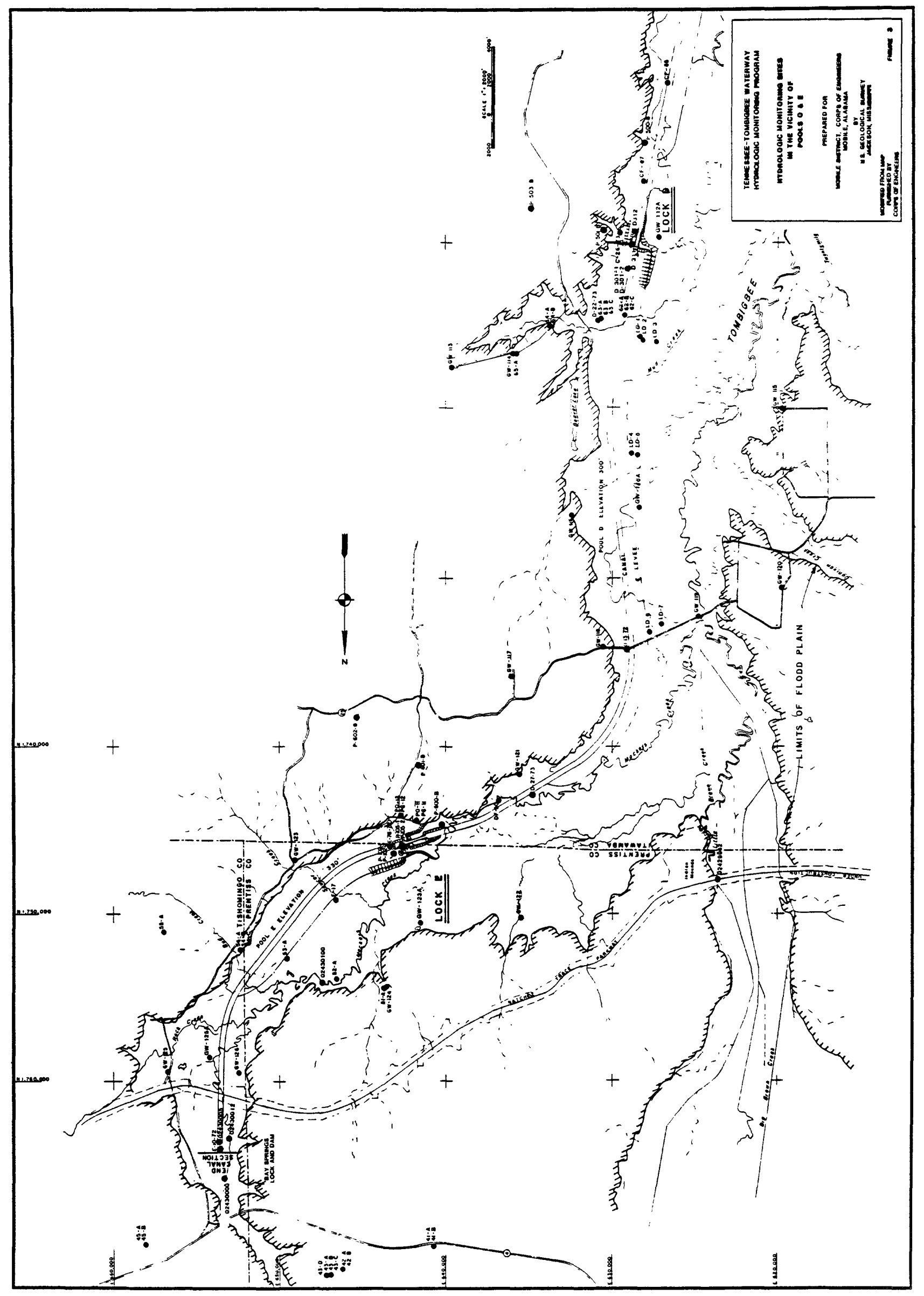




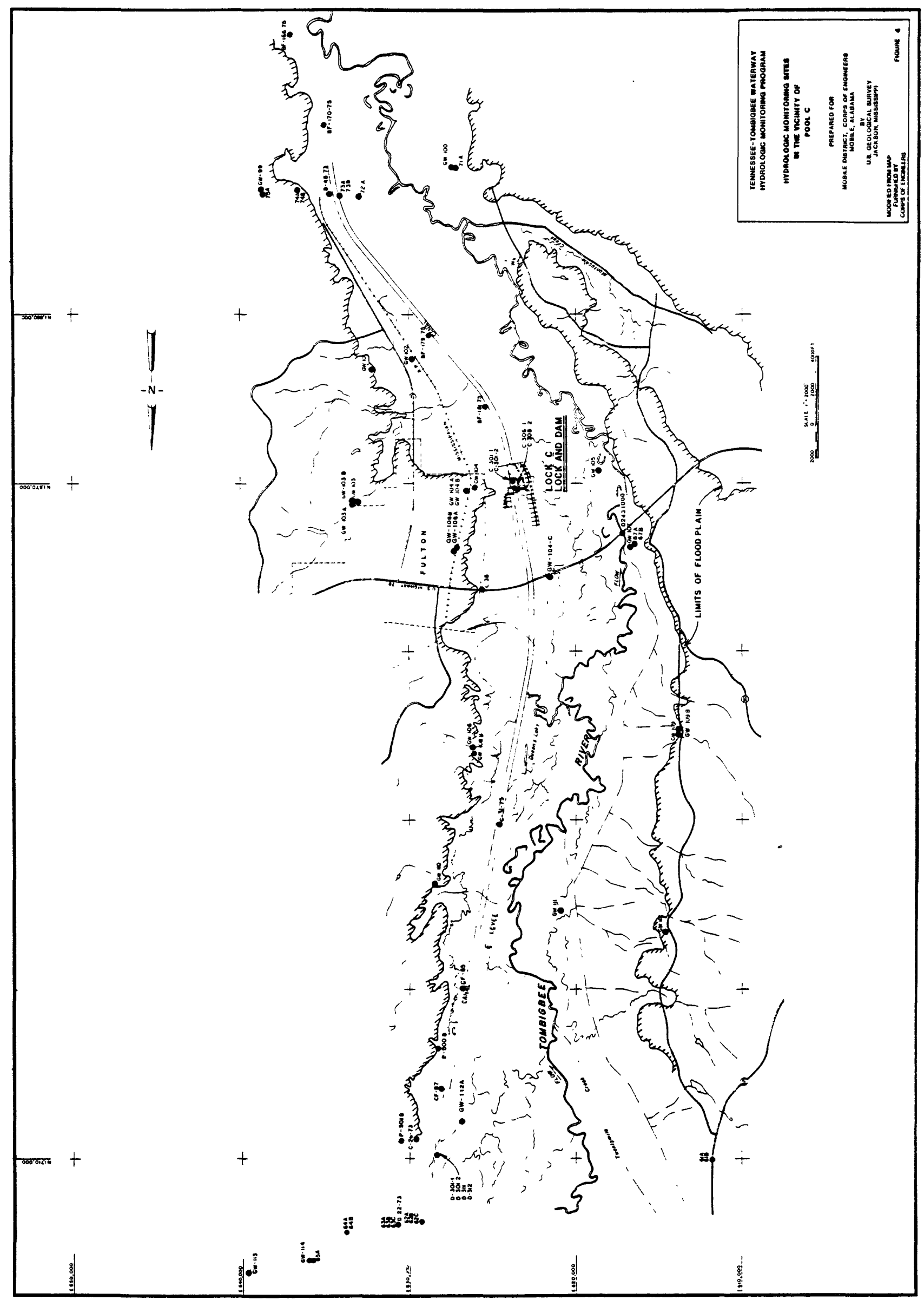




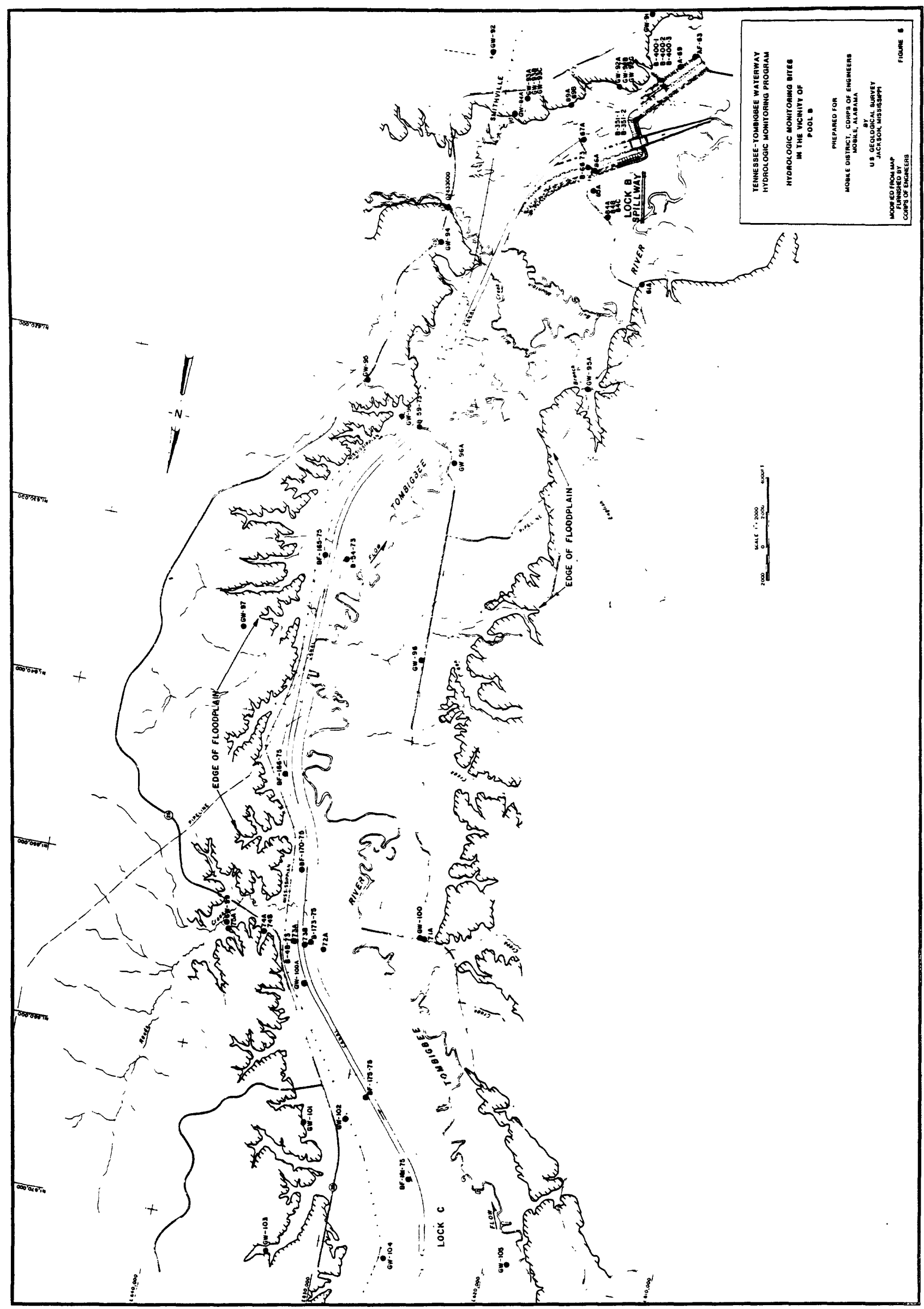




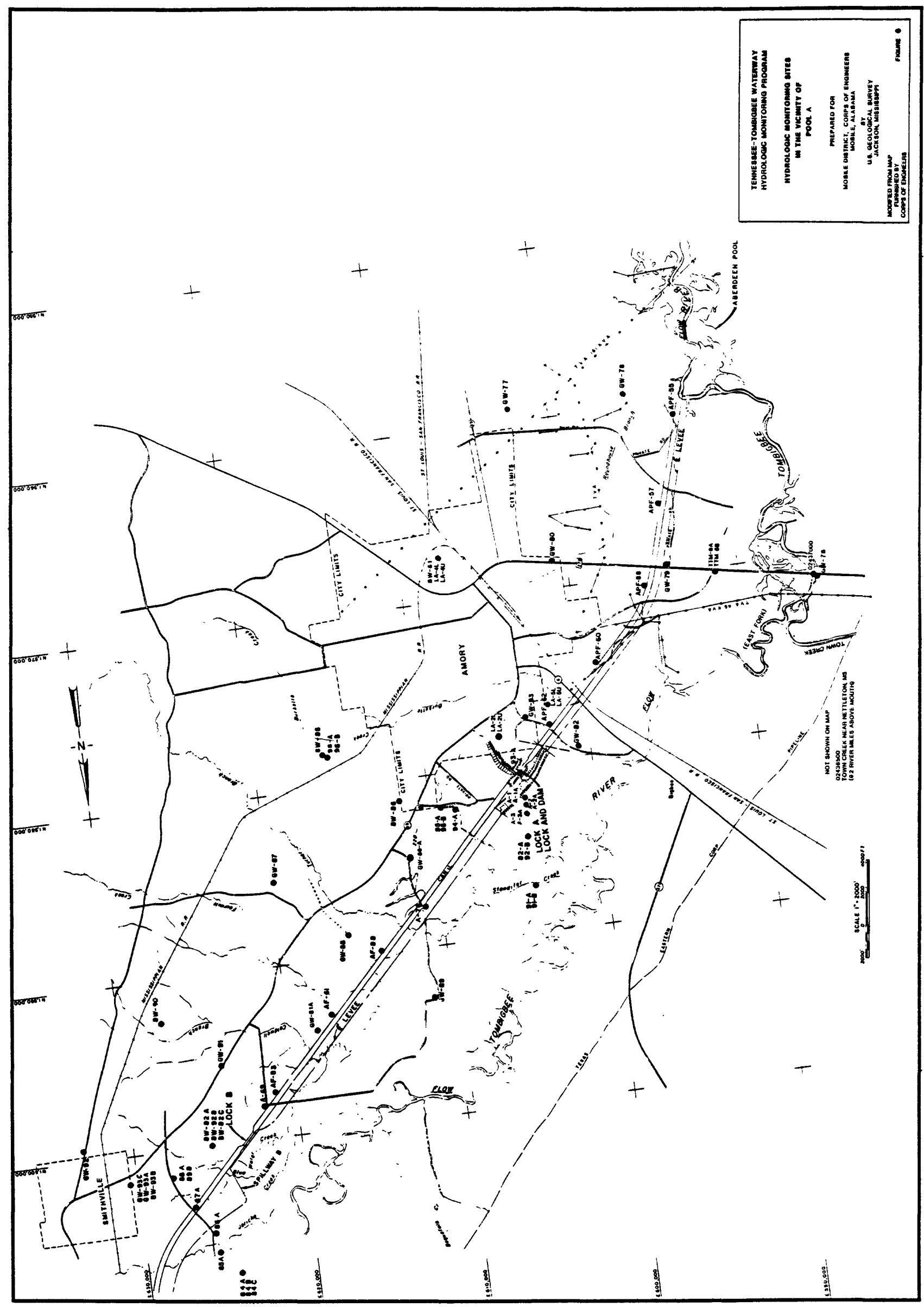




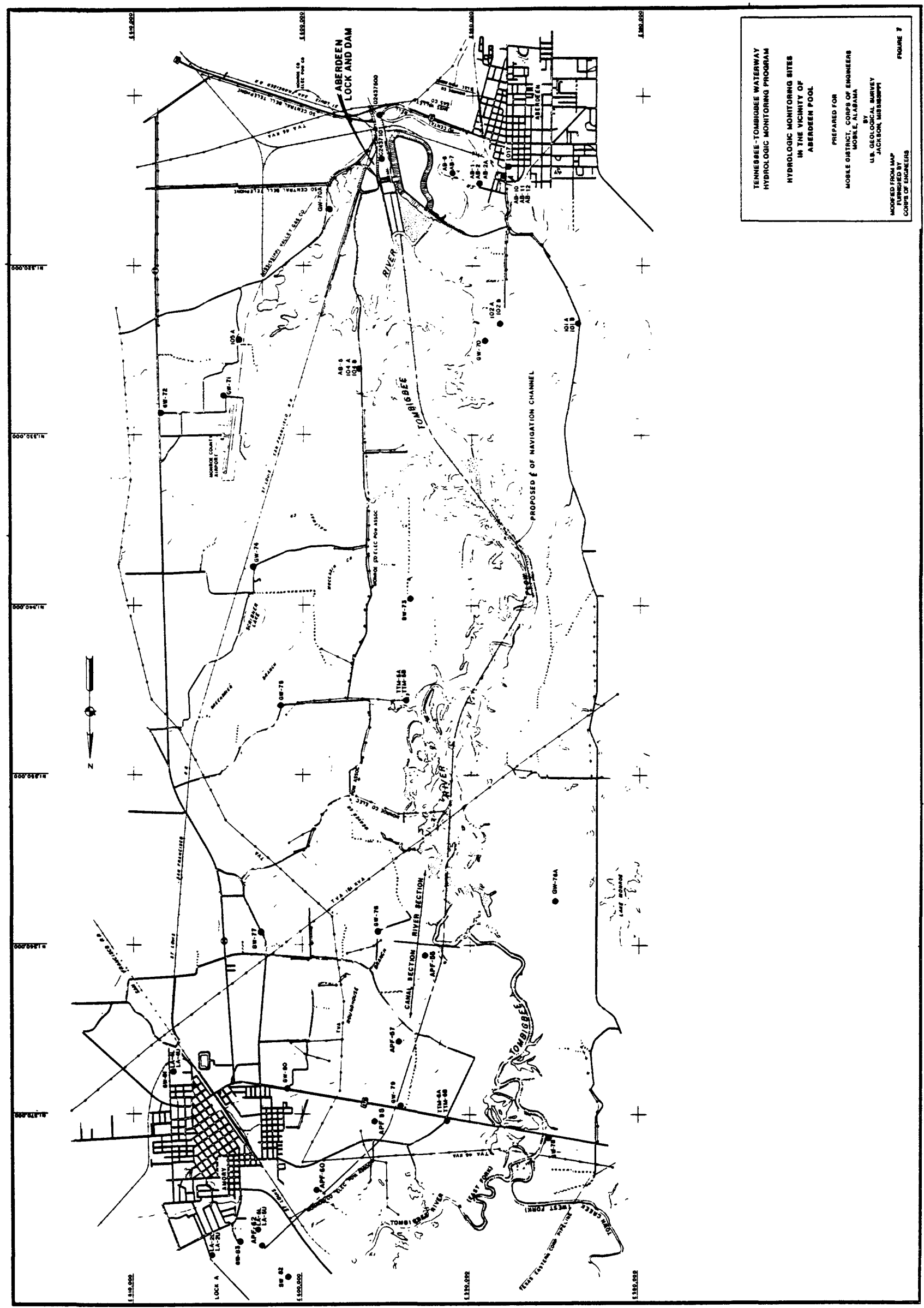




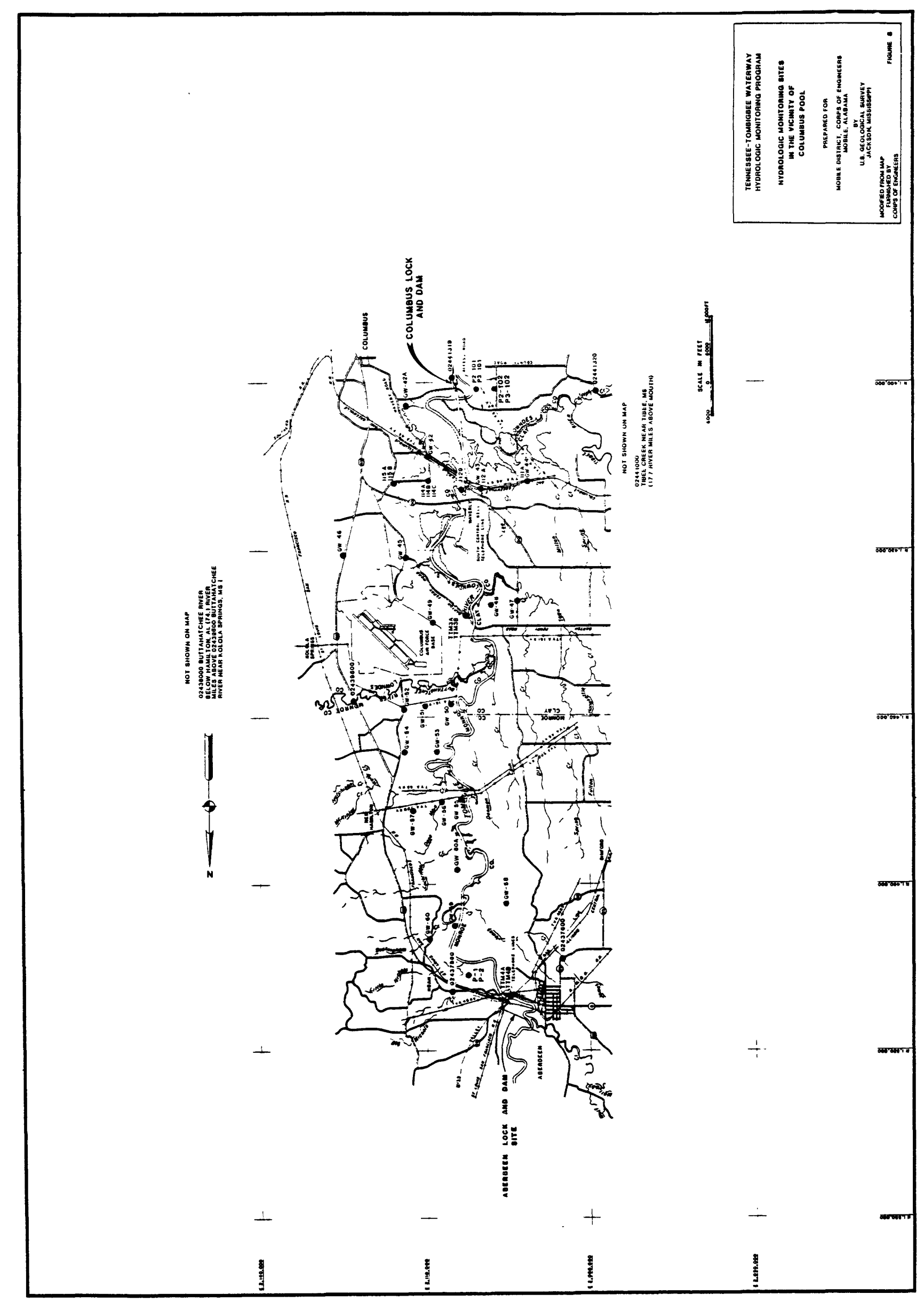




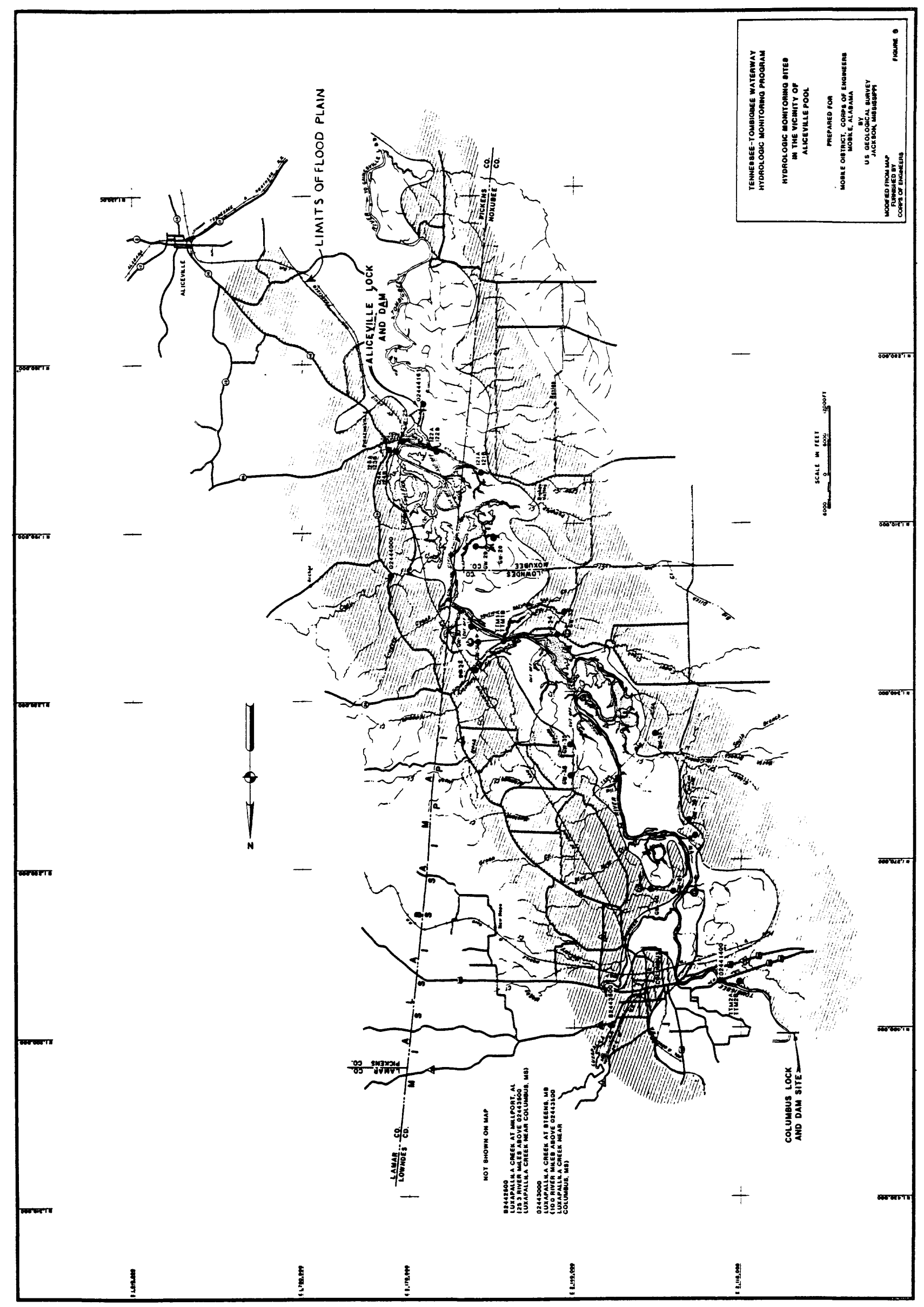




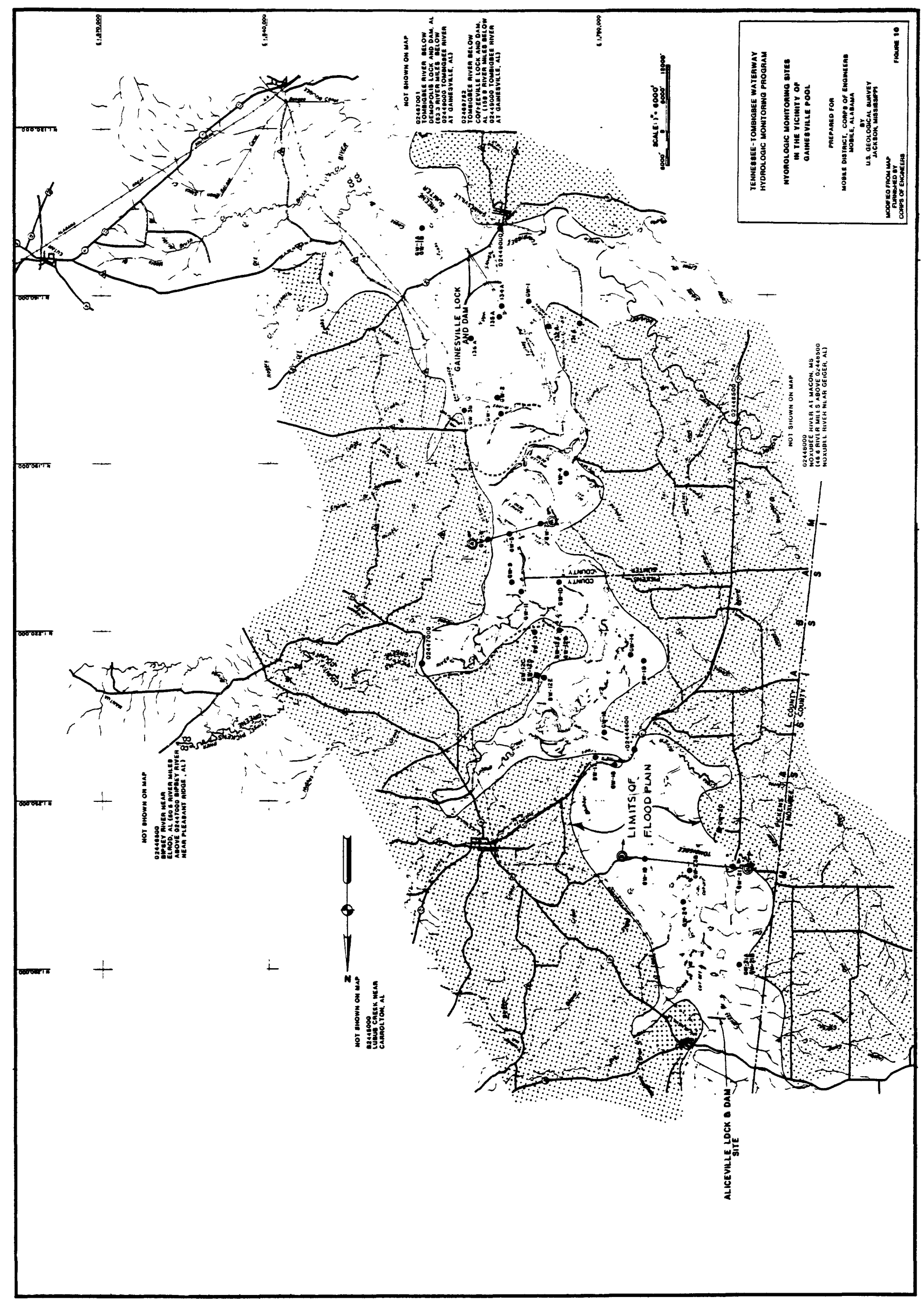




\section{Levels}

Under natural conditions, water levels in wells fluctuate seasonally and reflect recharge to and discharge from aquifers. Water-level fluctuations ranging from less than 1 foot to more than 10 feet per year have been observed in the aquifers in the study area. Water-level fluctuations generally were larger in the alluvial and terrace aquifers than in the regional aquifers.

During Fiscal Year 1990 (FY90), water levels in all 134 observation wells in the network were scheduled to be measured during the first quarter by the U.S. Geological Survey (USGS). However, one well (GW112) was destroyed and four (GW94, GW97, GW-111, and 6DP164) were dry or could not be measured because of obstructions in the wells. Hydrographs showing waterlevel variations in the wells for the period of USGS record are presented in Appendix A.

\section{Quality}

Water-quality data were collected at five wells selected by the COE. Results of the analyses are given in Appendix A.

\section{Surface Water}

\section{Network}

The surface-water network, which is designed to monitor water quality, currently consists of 15 sites in the area of the Tennessee-Tombigbee Waterway. Descriptions of these sites are tabulated in Appendix B.

\section{Stage and Discharge}

Surface-water stage and discharge data were collected at numerous sites (including most sites at which water-quality data were collected) in the area of the Tennessee-Tombigbee Waterway. The collection of stage and discharge data at these sites was funded by cooperative programs with various State and Federal agencies. Data collected at these sites are available either in the Jackson, Miss., or Tuscaloosa, Ala., office of the USGS. 


\section{Quality}

Water-quality data were collected by the USGS at 15 surface-water sites in the network during the 1990 reporting period (Appendix B).

Data for site 02433000, Bull Mountain Creek near Smithville, Miss., and site 02441000 , Tibbee Creek near Tibbee, Miss. may not represent water-quality conditions upstream of the sampling sites. Normal pool elevation of 245.00 feet above sea level for Pool B creates a stage of about 10.2 feet at the Bull Mountain Creek site and a normal pool elevation of 163.00 feet above sea level for Columbus Lake creates a stage of about 8.8 feet at the Tibbee Creek site, resulting in variable backwater conditions. Measurements of stream discharge were not obtained during backwater conditions.

Data for suspended-sediment concentration, particle-size distribution of suspended sediment, and particle-size distribution of stream bed material were collected at site 02436500 , Town Creek near Nettleton, Miss.; site 02437500, Tombigbee River at Aberdeen, Miss.; and site 02448000, Noxubee River at Macon, Miss. Data for particle-size distribution of stream bed material was collected at site 332929088273300, TTW Aliceville Lake above Columbus Bend; site 02441498, Tombigbee River Columbus Bend SR 11B at Columbus, Miss.; and site 332751088261000, TTW Aliceville Lake Columbus Cut. Data for suspended-sediment concentration was collected at the following sites:

$\begin{array}{ll}02430500 & \text { Tombigbee River near Marietta, Miss. } \\ 02430680 & \text { Twentymile Creek near Guntown, Miss. } \\ 02430690 & \text { Twentymile Creek near Mantachie, Miss. } \\ 02431000 & \text { Tombigbee River near Fulton, Miss. } \\ 02431410 & \text { Mantachie Creek below Dorsey, Miss. } \\ 02433500 & \text { Tombigbee River at Bigbee, Miss. } \\ 02437000 & \text { Tombigbee River near Amory, Miss. } \\ 02439400 & \text { Buttahatchee River near Aberdeen, Miss. } \\ 02441400 & \text { Tombigbee River near Columbus, Miss. } \\ 02443500 & \text { Luxapallila Creek near Columbus, Miss. } \\ 02444157 & \text { Tombigbee River at St Hwy 86 near Pickensville, Ala. } \\ 02444500 & \text { Tombigbee River near Cochrane, Ala. } \\ 02469525 & \text { Tombigbee River near Nanafalia, Ala. }\end{array}$

Miscellaneous samples were collected periodically at two sites on the lower Tombigbee River: 02449000 Tombigbee River at Gainesville, Ala. (monthly), and 02469762 Tombigbee River below Coffeeville Lock and Dam, Ala. (quarterly); specific conductance and water temperature were measured daily at site 02469762, Tombigbee River below Coffeeville Lock and Dam, Ala. 
The results of these USGS measurements and analyses are presented in Appendix B. Collection of these data was funded by cooperative agreements with various State and Federal agencies.

\section{QUALITY ASSURANCE}

\section{Ground-Water Levels}

The collection, analysis, and computation of ground-water level records are conducted in accordance with techniques and procedures established by the USGS and are within the guidelines recommended in the "National Handbook of Recommended Methods for Water-Data Acquisition" (Office of Water Data Coordination, 1977).

\section{Surface-Water Stage and Discharge}

The collection, analysis, and computation of surface-water stage and discharge records are conducted in accordance with procedures described in a series entitled "Techniques of Water-Resources Investigations of the U.S. Geological Survey" (TWRI). Field activities are presented in three chapters entitled "General Procedure for Gaging Streams" (Carter and Davidian, 1968); "Stage Measurements at Gaging Stations" (Buchanan and Somers, 1968); and "Discharge Measurements at Gaging Stations" (Buchanan and Somers, 1969); and more recently in Water-Supply Paper 2175, "Measurement and Computation of Streamflow: Volume 1, Measurement of Stage and Discharge" (Rantz and others, 1982). Daily discharge is computed in conformance with procedures described in Water-Supply Paper 2175, "Measurement and Computation of Streamflow: Volume 2, Computation of Discharge" (Rantz and others, 1982). All these procedures are within the guidelines recommended in the "National Handbook of Recommended Methods for Water-Data Acquisition" (Office of Water Data Coordination, 1977).

\section{Water Quality}

Procedures used by the USGS in the collection and analysis of samples of water and bottom materials are in conformance with the methods of laboratory analysis and sample preservation and handling described in TWRI Chapter A1, Book 5, "Methods for Determination of Inorganic Substances in Water and Fluvial Sediments" (Fishman and Friedman, 1989). 
Water samples collected as a part of this investigations were analyzed in one of the National Water-Quality Laboratories of the Water Resources Division, USGS. The National Water Quality Laboratories have an effective quality control program that includes the use of duplicate samples and standard reference water samples. TWRI "Quality Assurance Practices for the Chemical and Biological Analysis of Water and Fluvial Sediments" (Friedman and Erdmann, 1982) describes quality control techniques, quality assurance practices, and statistical techniques used by the National WaterQuality Laboratories.

The methods used in the collection and analyses of bacteriological samples are given in TWRI "Methods for Collection and Analysis of Aquatic Biological and Microbiological Samples" (Britton and Greeson, 1989). All bacteriological analyses were performed in the field within a few hours after the samples were collected.

The methods used in the collection and analyses of suspended-sediment samples are given in TWRI "Field Methods for Measurement of Fluvial Sediment" (Guy and Norman, 1970) and "Laboratory Theory and Methods for Sediment Analysis" (Guy, 1969).

Procedures used for water-quality field data collection are in accordance with techniques established by the USGS and are within the guidelines recommended in the "National Handbook of Recommended Methods for Water-Data Acquisition" (Office of Water Data Coordination, 1977). 


\section{SELECTED REFERENCES}

Brahana, J.V., German, E.R., Davis, M.E., and Wasson, B.E., 1974, Predicted effects of the Tennessee-Tombigbee Waterway on the hydrologic environment, Mississippi-Alabama in First supplemental environmental report, continuing environmental studies, Tennessee-Tombigbee Waterway, Alabama and Mississippi: U.S. Army Corps of Engineers, Mobile District, v. 2, 3, and 4.

Britton, L.J., and Greeson, P.E., editors, 1989, Methods for collection and analysis of aquatic biological and microbiological samples, Chapter A4 of Book 5, Laboratory analyses: U.S. Geological Survey Techniques of Water-Resources Investigations, $363 \mathrm{p}$.

Buchanan, T.J., and Somers, W.P., 1968, Stage measurements at gaging stations, Chapter A7 of Book 3, Applications of hydraulics: U.S. Geological Survey Techniques of Water-Resources Investigations, $28 \mathrm{p}$.

----1969, Discharge measurements at gaging stations, Chapter A8 of Book 3, Applications of hydraulics: U.S. Geological Survey Techniques of WaterResources Investigations, $65 \mathrm{p}$.

Carter, R.W., Davidian, Jacob, 1968, General procedure for gaging streams, Chapter A6 of Book 3, Applications of hydraulics: U.S. Geological Survey Techniques of Water-Resources Investigations, $13 \mathrm{p}$.

Fishman, M.J., and Friedman, L.C., 1989, Methods for determination of inorganic substances in water and fluvial sediments, Chapter A1 of Book 5, Laboratory analysis: U.S. Geological Survey Techniques of Water-Resources Investigations, $545 \mathrm{p}$.

Friedman, L.C., and Erdmann, D.E., 1982, Quality assurance practices for the chemical and biological analyses of water and fluvial sediments, Chapter A6 of Book 5, Laboratory analysis: U.S. Geological Survey Techniques of Water-Resources Investigations, $181 \mathrm{p}$.

Guy, H.P., 1969, Laboratory theory and methods for sediment analysis, Chapter C1 of Book 5, Laboratory analysis: U.S. Geological Survey Techniques of WaterResources Investigations, $58 \mathrm{p}$.

Guy, H.P., and Norman, V.W., 1970, Field methods for measurement of fluvial sediment, Chapter C2 of Book 3, Application of hydraulics: U.S. Geological Survey Techniques of Water-Resources Investigations, $59 \mathrm{p}$.

Leake, S.A., 1977, Simulation of flow to a partially penetrating trench: U.S. Geological Survey Journal of Research, v. 5, no. 5, 15 p. 
Morris, Fred, III, 1983, Hydrologic monitoring in the area of the TennesseeTombigbee Waterway, Mississippi-Alabama, Fiscal Year 1982: U.S. Geological Survey Open-File Report 83-764, 189 p.

---1984, Hydrologic monitoring in the area of the Tennessee-Tombigbee Waterway, Mississippi-Alabama, Fiscal Year 1983: U.S. Geological Survey Open-File Report 84-717, 174 p.

---1985, Hydrologic monitoring in the area of the Tennessee-Tombigbee Waterway, Mississippi-Alabama, Fiscal Year 1984: U.S. Geological Survey Open-File Report 85-692, $152 \mathrm{p}$.

---1986, Hydrologic monitoring in the area of the Tennessee-Tombigbee Waterway, Mississippi-Alabama, Fiscal Year 1985: U.S. Geological Survey Open-File Report 86-493, $311 \mathrm{p}$.

---1988, Hydrologic monitoring in the area of the Tennessee-Tombigbee Waterway, Mississippi-Alabama, Fiscal Year 1986: U.S. Geological Survey Open-File Report 88-321, 500 p.

--1991, Hydrologic monitoring in the area of the Tennessee-Tombigbee Waterway, Mississippi-Alabama, Fiscal Year 1987: U.S. Geological Survey Open-File Report 91-447, 245 p.

---1991, Hydrologic monitoring in the area of the Tennessee-Tombigbee Waterway, Mississippi-Alabama, Fiscal Year 1988: U.S. Geological Survey Open-File Report 91-511, $231 \mathrm{p}$.

---1991, Hydrologic monitoring in the area of the Tennessee-Tombigbee Waterway, Mississippi-Alabama, Fiscal Year 1989: U.S. Geological Survey Open-File Report 91-520, 142 p.

Office of Water Data Coordination, U.S. Geological Survey, 1977: National handbook of recommended methods for water-data acquisition.

Rantz, S.E., and others, 1982, Measurement and computation of streamflow: U.S. Geological Survey Water-Supply Paper 2175, v. 1 and 2, 631 p.

Seaber, P.R., Kapinos, F.P., and Knapp, G.L., 1987, Hydrologic unit maps: U.S. Geological Survey Water-Supply Paper 2294, 63 p. 
Shell, J.D., 1976, Hydrologic monitoring in the area of the Tennessee-Tombigbee Waterway, Mississippi-Alabama, July 1974 through June 1975: U.S. Geological Survey open-file report, $252 \mathrm{p}$.

--1977, Hydrologic monitoring in the area of the Tennessee-Tombigbee Waterway, Mississippi-Alabama, July 1975 through September 1976: U.S. Geological Survey open-file report, $227 \mathrm{p}$.

---1978, Hydrologic monitoring in the area of the Tennessee-Tombigbee Waterway, Mississippi-Alabama, Fiscal Year 1977: U.S. Geological Survey open-file report, $155 \mathrm{p}$.

--1979, Hydrologic monitoring in the area of the Tennessee-Tombigbee Waterway, Mississippi-Alabama, Fiscal Year 1978: U.S. Geological Survey open-file report, $198 \mathrm{p}$.

---1980, Hydrologic monitoring in the area of the Tennessee-Tombigbee Waterway, Mississippi-Alabama, Fiscal Year 1979: U.S. Geological Survey open-file report, $153 \mathrm{p}$.

Tomaszewski, D.J., 1981, Hydrologic monitoring in the area of the TennesseeTombigbee Waterway, Mississippi-Alabama, Fiscal Year 1980: U.S. Geological Survey Open-File Report 81-546, 142 p.

---1982, Hydrologic monitoring in the area of the Tennessee-Tombigbee Waterway, Mississippi-Alabama, Fiscal Year 1981: U.S. Geological Survey Open-File Report 82-503, $140 \mathrm{p}$. 


\section{APPENDIXES}




\section{PRINCIPAL AQUIFER}

Geologic

unit code

Aquifer name and age

11OALVM

11 OTRCS

211TBGB

21 UETW

211EUTW

$211 E U T W R$

211 MCSN

Uaternary alluvium, Quaternary

ndifferentiated terrace deposits, Quaternary

Tombigbee Sand Member of Eutaw Formation, Upper Cretaceous

Eutaw Formation, Upper Cretaceous

Eutaw Formation (Restricted), Upper Cretaceous

McShan Formation, Upper Cretaceous

Gordo Formation, Upper Cretaceous

HYDROLOGIC UNIT

An elght-digit hydrologic unit code refers to a specific drainage basin as delineated by the Office of Water Data Coordination on the State Hydrologic Untt Maps (Seaber and others, 1987).

WATER-QUALITY REMARKS

Remark Code

Remark

\begin{tabular}{ll} 
Remark Code & \multicolumn{2}{c}{ Remark } \\
\hline $\mathrm{E}$ & Estimated value \\
$>$ & Actual value is known to be greater than the value shown \\
$<$ & Actual value is known to be less than the value shown \\
$\mathrm{K}$ & $\begin{array}{l}\text { Results based on colony count outside the acceptance } \\
\text { range (non-ideal colony count) }\end{array}$
\end{tabular}

WATER-QUALITY SAMPLE SOURCE AND CONDITION

Numertcal codes have been assigned to describe the source of the sample and the condttions under which 1 t was collected. A numerical code will not be given in a water-quality table except to describe unusual conditions. The codes that may be given in this report are as follows:

Sample source

1 Well Head

26 Pump
Sampling condition

8 Pumping 


\section{APPENDIX A}

GROUND-WATER DATA

PAGE 27 FOLLOWS 


\section{APPENDIX A}

\section{GROUND-WATER DATA}

\section{DESCRIPTIONS OF WELLS}

PAGE 29 FOLLOW' 
IOCAL

LOCAL $\quad$ PRINCIPAL

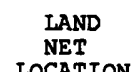

DATE

COMPLETED

$\begin{array}{cccc}\text { ALTITUDE } & & \text { CASING } & \text { DISCHARGE } \\ \text { OF LAND } & \text { DEPTH } & \text { DIAM- } & \text { (GALIONS } \\ \text { SURFACE } & \text { OF WEIL } & \text { ETER } & \text { PER } \\ \text { (FEET) } & \text { (FEET) } & \text { (INCHES) } & \text { MINUTE) }\end{array}$

GROUND-WATER NETWORK

ITANAMBA COUNTY

\begin{tabular}{|c|c|c|c|}
\hline $\begin{array}{l}\mathrm{A} 023 \\
\mathrm{~A} 024 \\
\mathrm{~A} 025 \\
\mathrm{~B} 005 \\
\mathrm{~B} 008\end{array}$ & $\begin{array}{l}\text { USCE } \\
\text { USCE } \\
\text { USCE } \\
\text { USCE } \\
\text { USCE }\end{array}$ & $\begin{array}{l}\text { GW118 } \\
\text { GW119 } \\
\text { GW120 } \\
\text { GW1117 } \\
\text { P601B }\end{array}$ & $\begin{array}{l}211 \mathrm{MCSN} \\
110 \mathrm{ALVM} \\
110 \mathrm{ALV} \\
110 \mathrm{ALV} \\
211 \mathrm{GORD}\end{array}$ \\
\hline $\begin{array}{l}\text { BO09 } \\
\text { D039 } \\
\text { DO } 40 \\
\text { DO } 41 \\
\text { D0 } 42\end{array}$ & $\begin{array}{l}\text { USCE } \\
\text { USCE } \\
\text { USCE } \\
\text { USCE } \\
\text { USCE }\end{array}$ & $\begin{array}{l}\text { P602B } \\
\text { CF87 } \\
\text { GW110 } \\
\text { GW112A } \\
\text { GW115 }\end{array}$ & $\begin{array}{l}\text { 211GORD } \\
211 \mathrm{EUTW} \\
110 \mathrm{ALVM} \\
110 \mathrm{ALVM} \\
110 \mathrm{ALVM}\end{array}$ \\
\hline $\begin{array}{l}\text { DO } 43 \\
\text { DO } 44 \\
\text { DO } 45 \\
\text { DO } 46 \\
\text { EO } 05\end{array}$ & $\begin{array}{l}\text { USCE } \\
\text { USCE } \\
\text { USCE } \\
\text { USCE } \\
\text { USCE }\end{array}$ & $\begin{array}{l}\text { GW116 } \\
\text { GW116A } \\
\text { P500B } \\
\text { P501B } \\
65 A\end{array}$ & $\begin{array}{l}211 \text { EUTW } \\
11 \text { OALVM } \\
211 \text { GORD } \\
211 \text { GORD } \\
211 \text { GORD }\end{array}$ \\
\hline $\begin{array}{l}\text { EOO9 } \\
\text { EO10 } \\
\text { EO11 } \\
\text { G065 } \\
\text { GO66 }\end{array}$ & $\begin{array}{l}\text { USCE } \\
\text { USCE } \\
\text { USCE } \\
\text { USCE } \\
\text { USCE }\end{array}$ & $\begin{array}{l}\text { GW113 } \\
\text { GW114 } \\
\text { P503B } \\
67 \mathrm{~A} \\
67 \mathrm{~B}\end{array}$ & $\begin{array}{l}110 \text { ALVM } \\
110 A L V M \\
211 \text { GORD } \\
211 \text { GORD } \\
211 \text { EUTW }\end{array}$ \\
\hline $\begin{array}{l}\text { G067 } \\
\text { G068 } \\
\text { G070 } \\
\text { G072 } \\
\text { G074 }\end{array}$ & $\begin{array}{l}\text { USCE } \\
\text { USCE } \\
\text { USCE } \\
\text { USCE } \\
\text { USCE }\end{array}$ & $\begin{array}{l}\text { GW106B } \\
\text { GW106A } \\
\text { GW104C } \\
\text { C38 } \\
\text { GW104A }\end{array}$ & $\begin{array}{l}211 \text { GORD } \\
110 \mathrm{ALVM} \\
110 \mathrm{ALVM} \\
110 \mathrm{ALVM} \\
211 \mathrm{GORD}\end{array}$ \\
\hline $\begin{array}{l}\text { G075 } \\
\text { G076 } \\
\text { G077 } \\
\text { G078 } \\
\text { G079 }\end{array}$ & $\begin{array}{l}\text { USCE } \\
\text { USCE } \\
\text { USCE } \\
\text { USCE } \\
\text { USCE }\end{array}$ & $\begin{array}{l}\text { GW104B } \\
\text { GW105 } \\
\text { GW107 } \\
\text { GW108 } \\
\text { GW108B }\end{array}$ & $\begin{array}{l}211 \text { GORD } \\
110 \mathrm{ALVM} \\
110 \mathrm{ALVM} \\
211 \mathrm{EUTW} \\
211 \mathrm{GORD}\end{array}$ \\
\hline $\begin{array}{l}\text { G080 } \\
\text { G081 } \\
\text { G082 } \\
\text { G083 } \\
\text { G084 }\end{array}$ & $\begin{array}{l}\text { USCE } \\
\text { USCE } \\
\text { USCE } \\
\text { USCE } \\
\text { USCE }\end{array}$ & $\begin{array}{l}\text { GW109 } \\
\text { GW109B } \\
\text { GW111 } \\
\text { GW112 } \\
\text { GW104 }\end{array}$ & $\begin{array}{l}110 \mathrm{ALVM} \\
211 \mathrm{GORD} \\
110 \mathrm{ALVM} \\
110 \mathrm{ALVM} \\
110 \mathrm{ALVM}\end{array}$ \\
\hline $\begin{array}{l}\text { KO } 39 \\
\text { KO } 41 \\
\text { KO 42 } \\
\text { LO14 } \\
\text { L016 }\end{array}$ & $\begin{array}{l}\text { USCE } \\
\text { USCE } \\
\text { USCE } \\
\text { USCE } \\
\text { USCE }\end{array}$ & $\begin{array}{l}71 \mathrm{~A} \\
\text { GW100 } \\
\text { BF } 179-75 \\
74 \mathrm{~A} \\
75 \mathrm{~A}\end{array}$ & $\begin{array}{l}211 G O R D \\
11 \text { OAVM } \\
211 \text { GORD } \\
211 \text { GORD } \\
211 \text { GORD }\end{array}$ \\
\hline $\begin{array}{l}\text { L017 } \\
\text { L021 } \\
\text { L022 } \\
\text { LO } 23 \\
\text { L024 }\end{array}$ & $\begin{array}{l}\text { USCE } \\
\text { USCE } \\
\text { USCE } \\
\text { USCE } \\
\text { USCE }\end{array}$ & $\begin{array}{l}72 A \\
\text { BF170-75 } \\
\text { BF173-75 } \\
\text { GW99 } \\
\text { GW1OOA }\end{array}$ & $\begin{array}{l}11 \text { OALVM } \\
211 E U T W \\
211 \text { GORD } \\
11 \text { OALVM } \\
110 A I V M\end{array}$ \\
\hline $\begin{array}{l}\text { LO2 } 25 \\
\text { LO2 } 26 \\
\text { NO2 } 28 \\
\text { NO2 } 29 \\
\text { O010 }\end{array}$ & $\begin{array}{l}\text { USCE } \\
\text { USCE } \\
\text { USCE } \\
\text { USCE } \\
\text { USCE }\end{array}$ & $\begin{array}{l}\text { GW101 } \\
\text { GW102 } \\
\text { 81A } \\
\text { GW95A } \\
\text { GW94 }\end{array}$ & $\begin{array}{l}110 A L V M \\
110 A I V M \\
211 \text { GORD } \\
110 A L V M \\
110 A I V M\end{array}$ \\
\hline $\begin{array}{l}0011 \\
0012 \\
0013 \\
0014 \\
0015\end{array}$ & $\begin{array}{l}\text { USCE } \\
\text { USCE } \\
\text { USCE } \\
\text { USCE } \\
\text { USCE }\end{array}$ & $\begin{array}{l}\text { GW95 } \\
\text { GW96 } \\
\text { GW96A } \\
\text { GW97 } \\
\text { GW98 }\end{array}$ & $\begin{array}{l}110 \mathrm{ALVM} \\
110 \mathrm{ALVM} \\
110 \mathrm{ALVM} \\
110 \mathrm{ALVM} \\
110 \mathrm{ALVM}\end{array}$ \\
\hline
\end{tabular}

SESES 34 TO7SROBE

SWSWS 29TO7SRO9E

SWSWS 21 TO7SRO 9E

NWNES 28TO7SRO 9 E

NWSES 25T08SR0 8

NENES12T09SR08E

NENWS 25T0 8SR08E

SESES10T08SR08E

SESWS06T08SR09E

SWSES01T08SRO 8 E

SWSES 25T08SR08

SESES 24T08SR08

NESES18T08SRO9E

SWNES17TO8SRO 9E

NESES18TO8SRO9E

NWNE S 30 TO 8 SRO 9

SWSES 27T09SR0 $8 E$

SWSES 27TO 9SRO8E

SWSES 25TO 9SRO8E

SWSES 25TO 9SR0 8E

NWSWS 26T0 9SR08E

SWNWS 25TO 9SRO $8 E$

NESWS 36 TO 9SRO 8 E

NESWS 36 TO 9SRO8E

NESES 34 TO $9 S R 08 E$

SWES 27 TO SSRO 8

NESWS13TO 9SRO 8E

NWSWS 15 TO 9SRO $8 E$

NWSWS15TO9SRO8E

SESWSO2TO 9SRO $8 E$

NWSWS 03TO9SR08E

SWNWS 36 TO 9SR08E

NENWS 24 T1 OSRO $8 E$

NENES $24 T 1$ OSRO 8

NENES 12TIOSRO 8

SWSWS $17 T 1 O S R O 9 E$

SWSES17TIOSRO9E

NWSES 18T1 OSRO 9E

NESESI 9T1OSRO9E

NESES18T1OSRO9E

SWSESI 7TIOSRO 9

NWNWS1 8T1OSRO 9E

SESES 01TIOSRO 8E

NESWS 26T11SRO8E

SENES 23T1 1SRO8E

SESES 2OTIISRO 9E

SWSWS17TIISRO 9E

NWSES18T11SRO 9

SWNWS 2OTIISRO $9 E$

SESWS14T1OSRO $9 E$

SWSWS 31 TIOSRO9E
SENES $36 T 07$ SRO $8 E$

SESWSO 6 T1OSRO $9 E$

$\begin{array}{llr}07 / 08 / 1975 & 325.70 & 23 \\ 07 / 10 / 1975 & 295 & 38 \\ 07 / 14 / 1975 & 297.20 & 21 \\ 07 / 16 / 1975 & 359.20 & 21 \\ 05 / 16 / 1978 & 440 & 187 \\ 06 / 23 / 1978 & 450 & 200 \\ 10 / 01 / 1975 & 271.10 & 66 \\ 07 / 02 / 1975 & 282.70 & 23 \\ 06 / 19 / 1975 & 275 & 28 \\ 07 / 15 / 1975 & 299.20 & 29 \\ 07 / 08 / 1975 & 333.50 & 30 \\ 06 / 17 / 1980 & 285 & 25 \\ 08 / 28 / 1978 & 290 & 124 \\ 05 / 01 / 1978 & 308.50 & 152 \\ 05 / 18 / 1972 & 325 & 130 \\ 07 / 07 / 1975 & 329.50 & 26 \\ 07 / 03 / 1975 & 311.70 & 12 \\ 05 / 15 / 1978 & 420 & 221 \\ 08 / 12 / 1975 & 270 & 179 \\ 08 / 13 / 1975 & 270 & 71 \\ 04 / 19 / 1978 & 284 & 175 \\ 04 / 191 / 1978 & 290 & 10 \\ 01 / 01 / 1980 & 260 & 24 \\ 04 / 12 / 1973 & 263.20 & 33 \\ 04 / 20 / 1978 & 290 & 88 \\ 04 / 20 / 1978 & 290 & 138 \\ 07 / 11 / 1975 & 254.60 & 29 \\ 07 / 102 / 1975 & 273.30 & 31 \\ 07 / 02 / 1975 & 284.50 & 14 \\ 04 / 27 / 1978 & 284.50 & 150 \\ 07 / 03 / 1975 & 278.20 & 24 \\ 05 / 105 / 1978 & 278.20 & 198 \\ 07 / 23 / 1975 & 270.90 & 35 \\ 09 / 01 / 1975 & 292.90 & 24 \\ 07 / 01 / 1975 & 240 & 26 \\ 06 / 20 / 1972 & 273 & 170 \\ 06 / 24 / 1975 & 269.20 & 21 \\ 01 / 14 / 1976 & 250 & 46 \\ 06 / 22 / 1972 & 270 & 150 \\ 06 / 22 / 1972 & 300 & 144 \\ 06 / 11 / 1972 & 249 & 21 \\ 08 / 20 / 1975 & 247.50 & 67 \\ 07 / 15 / 1975 & 252.20 & 51 \\ 06 / 26 / 1975 & 282.80 & 30 \\ 07 / 08 / 1980 & 255 & 32 \\ 06 / 26 / 1975 & 304.80 & 21 \\ 06 / 22 / 1975 & 277.10 & 21 \\ 07 / 13 / 1972 & 246 & 180 \\ 06 / 16 / 1975 & 242.40 & 35 \\ 06 / 18 / 1975 & 278 & 23 \\ 06 / 19 / 1975 & 325.40 & 29 \\ 06 / 20 / 1975 & 262.90 & 23 \\ 06 / 22 / 1975 & 238.60 & 24 \\ 06 / 25 / 1975 & 393.90 & 60 \\ 06 / 23 / 1975 & 267.20 & 23 \\ 0 / 10 & \end{array}$

267.20

MONROE COUNTY

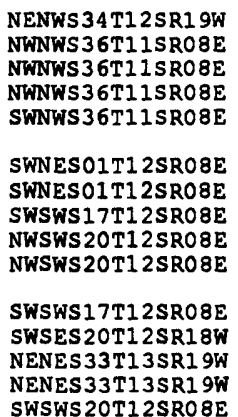

$01 / 15 / 1976$

$06 / 29 / 1972$

$07 / 10 / 1972$

$07 / 10 / 1972$

$07 / 11 / 1972$

$06 / 30 / 1972$

$07 / 06 / 1972$

$07 /--/ 1972$

$07 /--/ 1972$

$08 / 30 / 1972$

$08 /--11972$

$08 /--11972$

$05 / 14 / 1975$

$05 / 16 / 1975$

$06 / 17 / 1982$

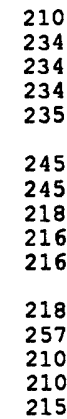

60
170
110
27
21
166
45
88
200
19
20
16
65
38
5

$\begin{array}{ll}1.50 & =- \\ 1.50 & =- \\ 1.50 & =- \\ 1.50 & =- \\ 1.50 & -- \\ 1.50 & =- \\ 1.50 & =- \\ 1.50 & =- \\ 1.50 & =- \\ 1.50 & -- \\ 1.50 & -- \\ 1.50 & =- \\ 1.50 & =- \\ 1.50 & 7.0\end{array}$

1.50 --

1.50
1.50

1.50

1.50

1.50
1.50

1.50

1.50

1.50

1.50

1.50
1.50

1.50

$\frac{1}{2}$

4

1.50

1.50

1.50
1.50

1.50
1.50

4.50

1.50
1.50

1.50

1.50

1.50

1.50

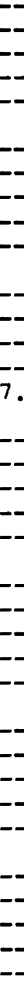

200

195

7.0
---
--
--
--
$\overline{8.0}$
--
--
--
--
--

211TBGB

11OALVM

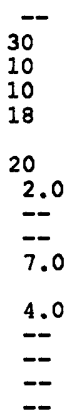


LOCAL

NUMBER
OWNER

PRINCIPAI AQUIFER

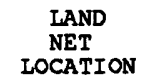

DATE

COMP LETED

ATTTUD OF IAND SURFACE SUREACE DEPTH DIAMOF WELL ETER
(FEET) (INCHES)
DISCHARGE

GALLONS

PER

GROUND-WATER NETWORK--COnt1 nued

MONROE COUNTY--Continued

\begin{tabular}{|c|c|c|c|}
\hline $\begin{array}{l}\text { C086 } \\
C 087 \\
C 088 \\
C 089 \\
\operatorname{cog} 9\end{array}$ & $\begin{array}{l}\text { USCE } \\
\text { USCE } \\
\text { USCE } \\
\text { USCE } \\
\text { USCE }\end{array}$ & $\begin{array}{l}\text { A1 } \\
\text { A2 } \\
\text { A2A } \\
\text { A3 } \\
\text { A3A }\end{array}$ & $\begin{array}{l}\text { 11OALVM } \\
110 A L V M \\
110 A L V M \\
110 A L V M \\
110 A L V M\end{array}$ \\
\hline $\begin{array}{l}C 092 \\
C 093 \\
C 094 \\
C 095 \\
C 096\end{array}$ & $\begin{array}{l}\text { USCE } \\
\text { USCE } \\
\text { USCE } \\
\text { USCE } \\
\text { USCE }\end{array}$ & $\begin{array}{l}\text { GW80 } \\
\text { GW81 } \\
\text { GW83 } \\
\text { GW85 } \\
\text { GWB6 }\end{array}$ & $\begin{array}{l}110 \mathrm{ALVM} \\
110 \mathrm{ALVM} \\
110 \mathrm{ALVM} \\
110 \mathrm{ALVM} \\
110 \mathrm{ALVM}\end{array}$ \\
\hline $\begin{array}{l}\text { CO97 } \\
\text { CO98 } \\
\text { C099 } \\
\text { C100 } \\
\text { C101 }\end{array}$ & $\begin{array}{l}\text { USCE } \\
\text { USCE } \\
\text { USCE } \\
\text { USCE } \\
\text { USCE }\end{array}$ & $\begin{array}{l}\text { GW86A } \\
\text { GW87 } \\
\text { GW88 } \\
\text { GW89 } \\
\text { GW90 }\end{array}$ & $\begin{array}{l}110 \mathrm{ALVM} \\
110 \mathrm{ALVM} \\
110 \mathrm{ALVM} \\
110 \mathrm{ALVM} \\
110 \mathrm{ALVM}\end{array}$ \\
\hline $\begin{array}{l}\text { C102 } \\
\text { C105 } \\
\text { C106 } \\
\text { C107 } \\
\text { C108 }\end{array}$ & $\begin{array}{l}\text { USCE } \\
\text { USCE } \\
\text { USCE } \\
\text { USCE } \\
\text { USCE }\end{array}$ & $\begin{array}{l}\text { GW91 } \\
\text { GW92B } \\
\text { GW92C } \\
\text { LA2L } \\
\text { LA2U }\end{array}$ & $\begin{array}{l}110 \text { ALVM } \\
211 \text { EUTW } \\
211 \text { EUTW } \\
211 \text { GORD } \\
211 \text { EUTW }\end{array}$ \\
\hline $\begin{array}{l}\text { C109 } \\
\text { C1110 } \\
\text { C1111 } \\
\text { C1112 } \\
\text { C1113 }\end{array}$ & $\begin{array}{l}\text { USCE } \\
\text { USCE } \\
\text { USCE } \\
\text { USCE } \\
\text { USCE }\end{array}$ & $\begin{array}{l}\text { LA4L } \\
\text { LA4U } \\
\text { LA5L } \\
\text { LA5U } \\
\text { AF } 63\end{array}$ & $\begin{array}{l}211 \mathrm{GORD} \\
211 \mathrm{EUTW} \\
211 \mathrm{GORD} \\
211 \mathrm{EUTW} \\
211 \mathrm{EUTW}\end{array}$ \\
\hline $\begin{array}{l}\text { D032 } \\
\text { D033 } \\
\text { D034 } \\
\text { D035 } \\
\text { D036 }\end{array}$ & $\begin{array}{l}\text { USCE } \\
\text { USCE } \\
\text { USCE } \\
\text { USCE } \\
\text { USCE }\end{array}$ & $\begin{array}{l}\text { GW92 } \\
\text { GW93A } \\
\text { GW93B } \\
\text { GW93C } \\
\text { GW94A }\end{array}$ & $\begin{array}{l}110 \mathrm{ALVM} \\
211 \mathrm{GORD} \\
211 \mathrm{EUTW} \\
110 \mathrm{ALVM} \\
110 \mathrm{ALVM}\end{array}$ \\
\hline $\begin{array}{l}\text { G052 } \\
\text { G053 } \\
\text { G059 } \\
\text { L095 } \\
\text { L096 }\end{array}$ & $\begin{array}{l}\text { USCE } \\
\text { USCE } \\
\text { USCE } \\
\text { USCE } \\
\text { USCE }\end{array}$ & $\begin{array}{l}\text { APF55 } \\
\text { APF 57 } \\
\text { GW78 } \\
\text { P1 } \\
\text { P2 }\end{array}$ & $\begin{array}{l}211 \text { EUTW } \\
211 E U T W \\
110 A L V M \\
211 E U T W \\
211 E U T W\end{array}$ \\
\hline
\end{tabular}

NWNWS2 9T1 2SRO 8E

NWNWS 29T1 2SRO 8E

NWNWS 29T1 2SRO8E

\section{SWNES35T12SR1 9W}

NESES 36T1 2SR1 9W

SESES $30 T 12$ 2SROBE

SWSES2OT12SR18W

NESWS 21T1 2SRO8E

SENWS16T1 2SR18W

SWSWS15T12SROBE

SESWSO9T1 2SR08E

NENWS13T1 2SRO8E

SENES11T1 2SRO8E

NWSWS01T12SRO8E

ITI

SWNWS24T12SRI 9W

NESES36T12SR19W

NESES36T1 2SR1 9W

SWSES30T12SR08E

SWSES30T1 2SRO8E

SWSESO3T12SR08E

SWNWS06T12SRO9E

SWNWSO 6T1 2SRO 9E

SWNWS06T1 2SRO9E

NWNWSO6T1 2SRO 9E

NESWS03T13SR19W

SESWS 34T12SR1 9W

NENWS03T13SRO7E

NENWS35T1 4SR19W

NENWS 35T1 4SR19W
SWSWS 20T1 2SRO8E

NWNES19T1 2SR18W

NESWSO6T12SRO9E

\section{$06 / 17 / 1982$ \\ $06 / 18 / 198$ \\ $06 / 18 / 198$ \\ $06 / 21 / 1982$}

$05 / 28 / 1975$

$05 / 29 / 1975$

$06 / 02 / 1975$

$06 / 10 / 1975$

$06 / 09 / 1975$

$06 / 23 / 1980$

$06 / 18 / 1975$

$06 / 05 / 1975$

$06 / 04 / 1975$

$06 / 13 / 1975$

$06 / 12 / 1975$

$04 / 14 / 1975$

$04 / 14 / 1975$

$05 / 01 / 1975$

$05 / 02 / 1975$

05/03/1975

$05 / 04 / 1975$

$05 / 05 / 1975$

$05 / 06 / 1975$

$01 / 20 / 1976$

$06 / 11 / 1975$

$05 / 15 / 1975$

$05 / 15 / 1975$

$05 / 15 / 1975$

$08 / 05 / 1975$

$08 / 05 / 1975$

$07 / 22 / 1975$

$10 / 05 / 198$

$10 / 01 / 1982$

PRENTISS COUNTY

$\begin{array}{lll}\text { MO16 } & \text { USCE } 53 A & 211 \text { GORD } \\ \text { MO19 } & \text { USCE 52A } & 211 \text { GORD } \\ \text { MO20 } & \text { USCE 51A } & 211 \text { GORD } \\ \text { M028 } & \text { USCE GW123A } & \text { 11OTRCS } \\ \text { M030 } & \text { USCE GW122 } & 110 \text { ALVM } \\ & & \\ \text { M031 } & \text { USCE GW123 } & 11 \text { OALVM } \\ \text { M032 } & \text { USCE GW124 } & 110 A L V M\end{array}$

NWSES10T07SRO

NESES09T07SRO9E

SENWS09T07SRO9E

NWNWS 16T07SR0 9E

SWSES07TO7SR09E

$05 /--11972$

$05 /--/ 197$

$01 / 01 / 1980$

$02 / 21 / 1975$

$07 / 15 / 1975$

$07 / 18 / 1975$

SENWTISRO9E

TISHMINGO COUNTY

\begin{tabular}{|c|c|c|c|}
\hline $\begin{array}{l}\text { A017 } \\
D 047 \\
D 048 \\
D 055 \\
D 056\end{array}$ & $\begin{array}{l}\text { USCE } \\
\text { USCE } \\
\text { USCE } \\
\text { USCE } \\
\text { USCE }\end{array}$ & $\begin{array}{l}\text { 1ODP } 177 \\
\text { 1DP141 } \\
\text { 1DP142 } \\
\text { 3DP151 } \\
\text { 3DP152 }\end{array}$ & $\begin{array}{l}211 \text { GORD } \\
211 \text { ETWR } \\
211 \text { EUTWR } \\
211 \text { GORD } \\
211 \text { EUTW }\end{array}$ \\
\hline $\begin{array}{l}\text { D059 } \\
\text { D060 } \\
\text { G079 } \\
\text { G080 } \\
\text { G112 }\end{array}$ & $\begin{array}{l}\text { USCE } \\
\text { USCE } \\
\text { USCE } \\
\text { USCE } \\
\text { USCE }\end{array}$ & $\begin{array}{l}\text { 7DP1 } 67 \\
\text { 7DP168 } \\
\text { 6DP1 } 163 \\
\text { 6DP1 } 64 \\
\text { 2DP147 }\end{array}$ & $\begin{array}{l}211 \mathrm{GORD} \\
211 \mathrm{EUTW} \\
211 \mathrm{GORD} \\
211 \mathrm{EUTW} \\
211 \mathrm{GORD}\end{array}$ \\
\hline $\begin{array}{ll}\text { G1 } & 13 \\
\text { G1 } & 16 \\
\text { G1 } 18 \\
\text { G1 } \\
\text { G1 } 122 \\
\end{array}$ & $\begin{array}{l}\text { USCE } \\
\text { USCE } \\
\text { USCE } \\
\text { USCE } \\
\text { USCE }\end{array}$ & $\begin{array}{l}\text { 2DP1 } 48 \\
\text { 4DP156 } \\
\text { 4DP158 } \\
\text { 9DP173 } \\
\text { 9DP174 }\end{array}$ & $\begin{array}{l}211 \text { EUTW } \\
211 \text { GORD } \\
211 \text { EUTW } \\
211 \text { GORD } \\
211 \text { EUTW }\end{array}$ \\
\hline $\begin{array}{l}\text { J076 } \\
\text { J077 } \\
\text { L029 } \\
\text { L030 } \\
\text { L033 }\end{array}$ & $\begin{array}{l}\text { USCE } \\
\text { USCE } \\
\text { USCE } \\
\text { USCE } \\
\text { USCE }\end{array}$ & $\begin{array}{l}\text { 5DP1 } 59 \\
5 D P 160 \\
54 A \\
55 A \\
54 B\end{array}$ & $\begin{array}{l}211 \mathrm{GORD} \\
211 \mathrm{EUTW} \\
211 \mathrm{GORD} \\
211 \mathrm{GORD} \\
110 \mathrm{AIVM}\end{array}$ \\
\hline $\begin{array}{l}\text { L034 } \\
\text { L051 }\end{array}$ & $\begin{array}{l}\text { USCE } \\
\text { USCE }\end{array}$ & $\begin{array}{l}54 C \\
\text { GW125 }\end{array}$ & $\begin{array}{l}110 \mathrm{ALVM} \\
110 \mathrm{ALVM}\end{array}$ \\
\hline
\end{tabular}

NENHS 36T02SRO 9E

SENWS 35TO3SRO 9E

SENWS35TO3SRO9E

NESES14T03SRO9E

NESES14T03SRO9E

NESES01T03SRO9E

NESESO1T03SRO 9E

SESES 24T0 4SRO 9E

SESES24T0 4SRO 9E

SESWS11T04SRO 9E

SESWS11T04SRO9E

SESWS 31TO 4SR1 OE

SESWS 31 T0 4SR1 OE

NESES 14T0 4SRO9E

NESES14T04SRO 9E

NESWS08TO5SR10E

NESWSOBT0 5SR1OE

SWSWS11T07SRO9E

SESES11T07SRO 9E

SWSWS11TO7SRO9E

SWSWS11T07SRO9E

SENESO2T07SRO 9E
$05 / 14 / 1973$

$05 /=-1197$

$05 /--11972$

$08 / 30 / 1972$

$09 / 11 / 1972$

$03 / 12 / 1973$

$02 / 20 / 1973$

$02 / 23 / 1973$

$07 / 20 / 1972$

$07 / 25 / 1972$

$01 / 01 / 1973$

$10 / 26 / 1972$

$04 / 19 / 1973$

$11 / 06 / 1972$

$11 / 08 / 1972$

$04 / 28 / 1972$

$05 / 11 / 1972$

$06 / 06 / 1972$

05/01/1972

$07 / 22 / 1975$
$03 / 13 / 1973$

215
215
215
215
215

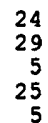

$\begin{array}{ll}1.50 & =- \\ 1.50 & = \\ 1.50 & = \\ 1.50 & = \\ 1.00 & =\end{array}$

236.40

235.70

213.20

257.60

247.10

210
258

244.20

244.20
225.20

225.2
270

261.30

257.30

257.40

220

220

\section{235}

210

210
230

266.70

259.20

259.40

259.40

255

205.70

207.30

205.30

190
190

1.50

1.50

1.50

1.50
1.50

1.50

1.50
1.50

1.50

1.50
1.50

1.50

1.50

1.50

1.50
1.50

1.50

1.50
1.50

1.50

1.50

1.50

1.50
1.50

1.50

1.50

1. 50

1.50
1.50

1.50

1.50

332

324

356
316

330

354

350

3.

6.0

$--$

$-$

433.40

464.50

462

453.30

453.30
451.40

447

446.60

573

572.60

572.60
504.50

504.70

504.70
490.10

487.70

552.20

552.40

437.20

436

332

380
332

333

367.10

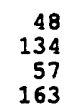

$\frac{3}{1} .50$

2.0

163

100

3

$=$

88
43
222

222
125

165

130

153

56
220

163

148

60
27
50

3
4
4
4
3

3.

3.5
4
4

13
17

$=$


DESCRIPTIONS OF GROUND-WATER WELLS--Cont1nUed

\begin{tabular}{|c|c|c|c|c|c|c|c|c|c|c|}
\hline \multirow[t]{2}{*}{$\begin{array}{l}\text { IOCAL } \\
\text { NUMBER }\end{array}$} & \multirow{2}{*}{\multicolumn{2}{|c|}{ OWNER }} & \multirow[t]{2}{*}{$\begin{array}{l}\text { PRINCIPAL } \\
\text { AQUIFER }\end{array}$} & & $\begin{array}{c}\text { LAND } \\
\text { NET } \\
\text { LOCATION }\end{array}$ & $\begin{array}{c}\text { DATE } \\
\text { COMPLETED }\end{array}$ & \multirow[t]{2}{*}{$\begin{array}{l}\text { ALTITUDE } \\
\text { OF LAND } \\
\text { SURFACE } \\
\text { (FEET) }\end{array}$} & \multirow[t]{2}{*}{$\begin{array}{l}\text { DEPTH } \\
\text { OF WELL } \\
\text { (FEET) }\end{array}$} & \multirow[t]{2}{*}{$\begin{array}{l}\text { CASING } \\
\text { DIAM- } \\
\text { ETER } \\
\text { (INCHES) }\end{array}$} & \multirow[t]{2}{*}{$\begin{array}{r}\text { DISCHA } \\
\text { (GALI } \\
\text { PER } \\
\text { MINUT }\end{array}$} \\
\hline & & & & \multicolumn{3}{|c|}{ PRENTISS COUNTY } & & & & \\
\hline $\begin{array}{l}\text { M017 } \\
\text { M018 } \\
\text { MO21 } \\
\text { MO22 } \\
\text { MO25 }\end{array}$ & $\begin{array}{l}\text { USCE } \\
\text { USCE } \\
\text { USCE } \\
\text { USCE } \\
\text { USCE }\end{array}$ & $\begin{array}{l}43 C \\
43 B \\
41 A \\
41 B \\
43 D\end{array}$ & $\begin{array}{l}\text { 211EUTWR } \\
\text { 211MCSN } \\
\text { 211GORD } \\
\text { 211EUTW } \\
\text { 211EUTWR }\end{array}$ & $\begin{array}{l}\text { NWNWS 27 } \\
\text { NWNWS 27 } \\
\text { SENWS } 28 \\
\text { SENWS } 28 \\
\text { NWNWS } 27\end{array}$ & $\begin{array}{l}\text { 7TO6SR09E } \\
\text { 7T06SR09E } \\
\text { 8T06SR09E } \\
\text { 8T06SR09E } \\
\text { 7T06SR09E }\end{array}$ & $\begin{array}{l}05 /--/ 1972 \\
05 /--/ 1972 \\
05 /--/ 1972 \\
05 /--/ 1972 \\
05 /--/ 1975\end{array}$ & $\begin{array}{l}445 \\
460 \\
480 \\
480 \\
460\end{array}$ & $\begin{array}{r}90 \\
120 \\
226 \\
176 \\
118\end{array}$ & $\begin{array}{l}4 \\
4 \\
4 \\
4 \\
4\end{array}$ & $\overline{--}$ \\
\hline
\end{tabular}




\section{APPENDIX A}

GROUND-WATER DATA

HYDROGRAPHS

PAGE 35 FOLLOWS 


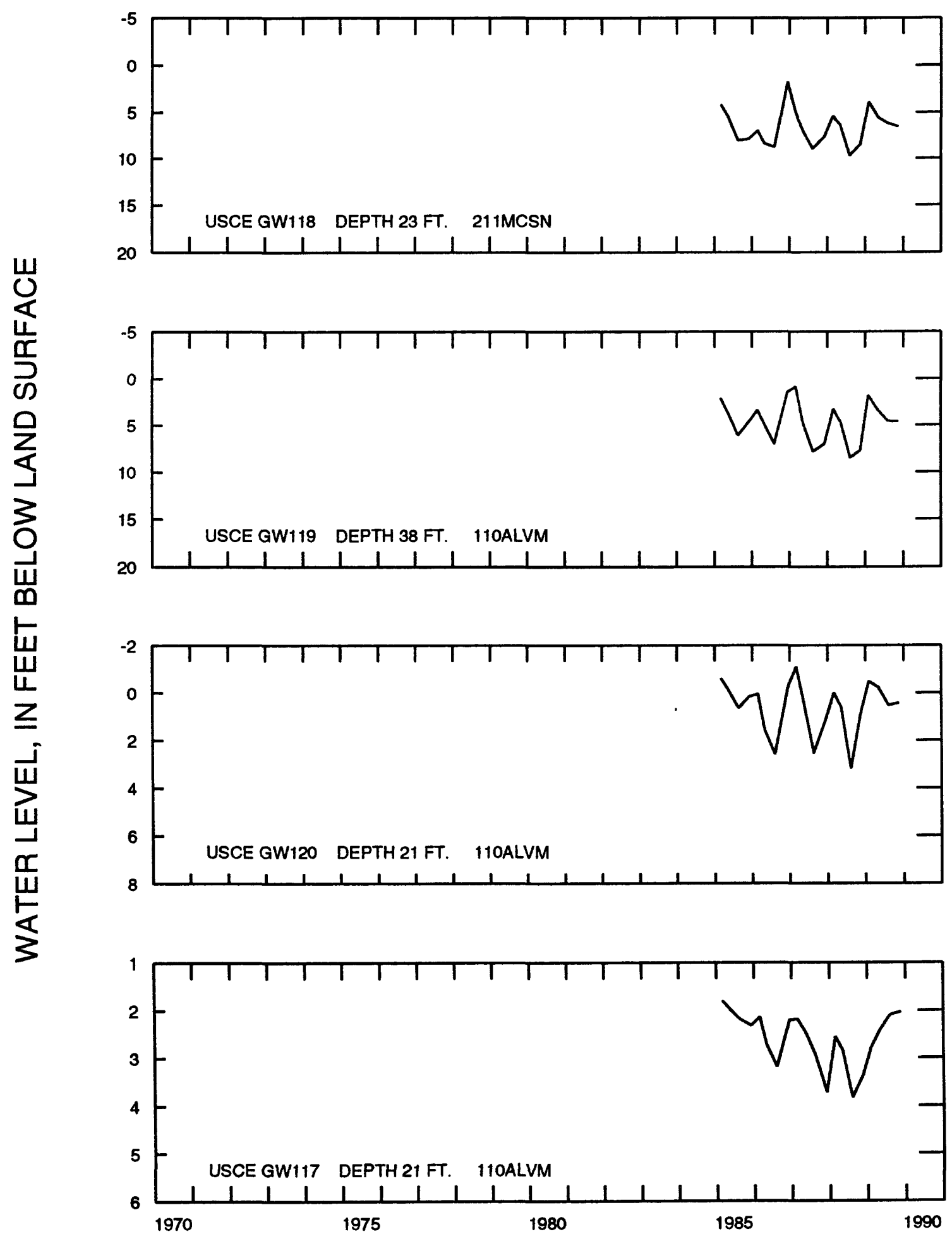

HYDROGRAPHS OF TENNESSEE-TOMBIGBEE OBSERVATION WELLS 


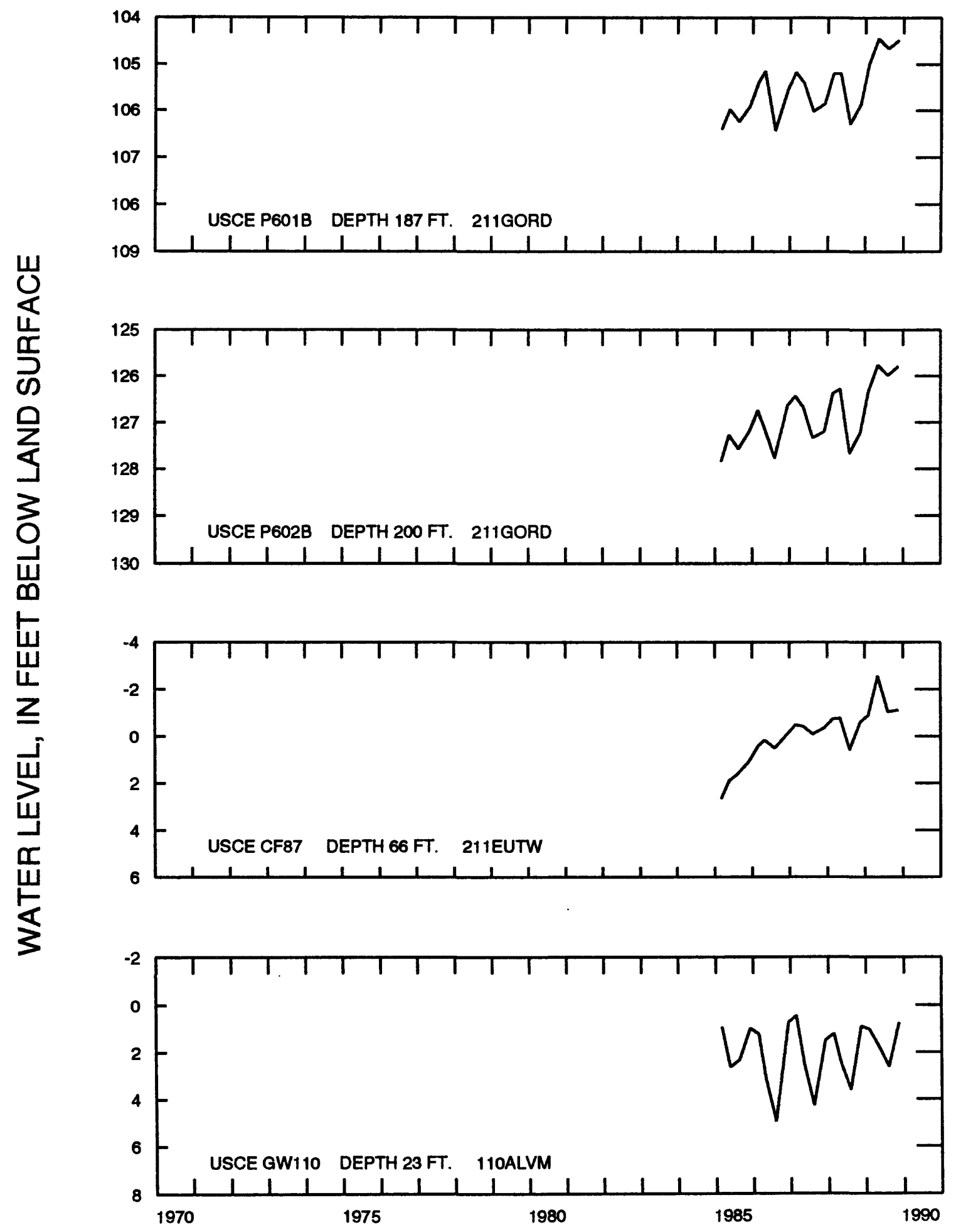

HYDROGRAPHS OF TENNESSEE-TOMBIGBEE OBSERVATION WELLS 


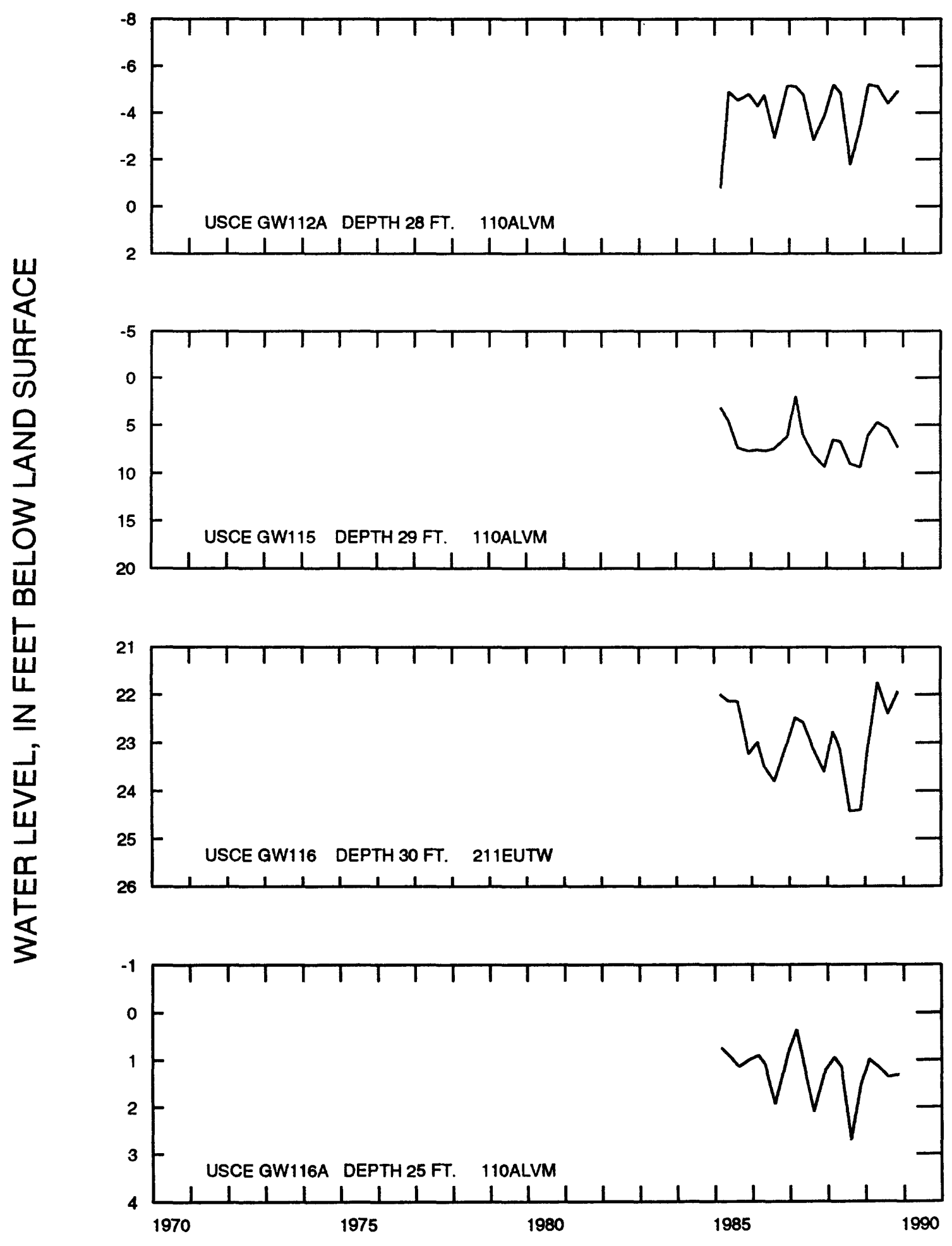

HYDROGRAPHS OF TENNESSEE-TOMBIGBEE OBSERVATION WELLS 


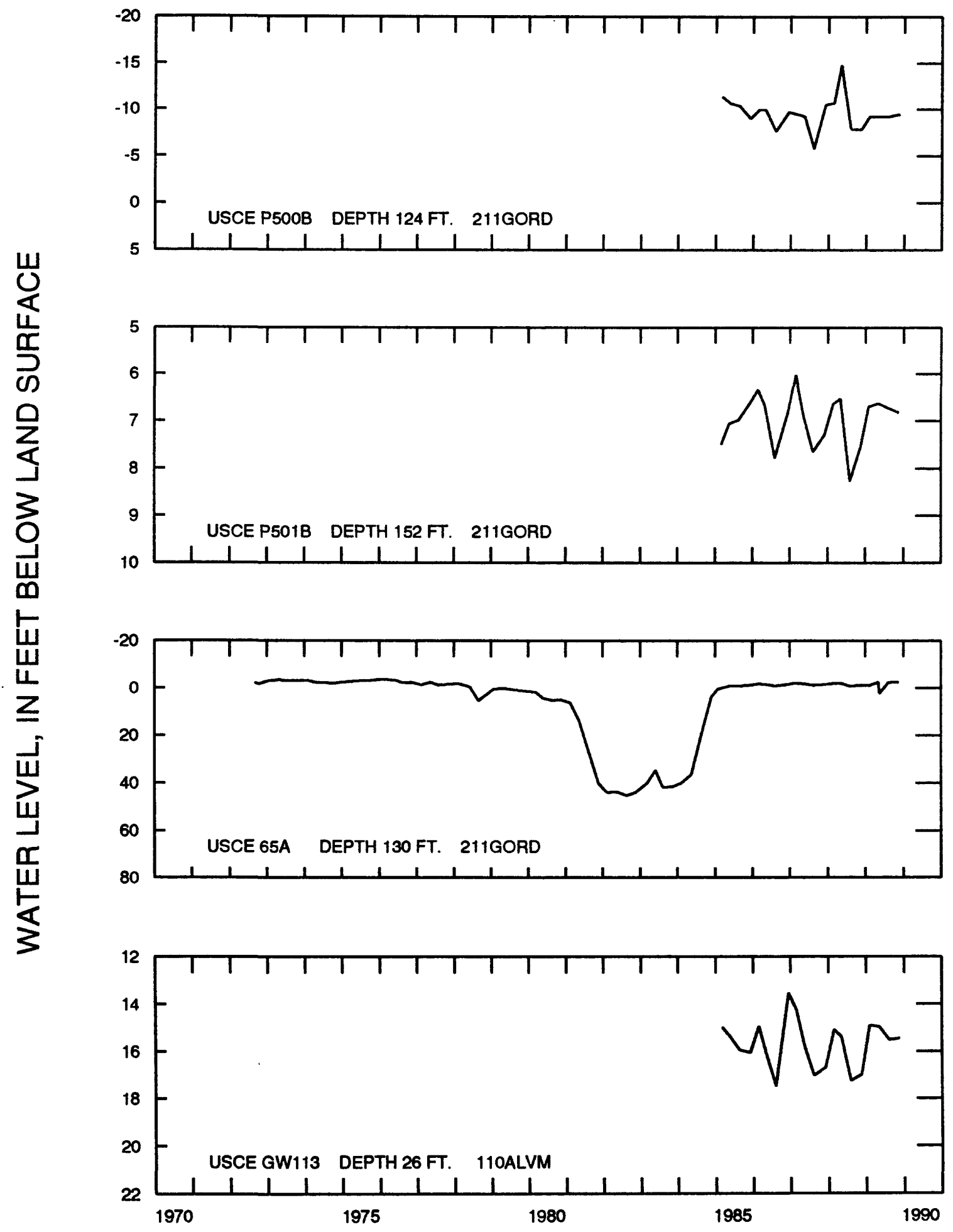

HYDROGRAPHS OF TENNESSEE-TOMBIGBEE OBSERVATION WELLS 


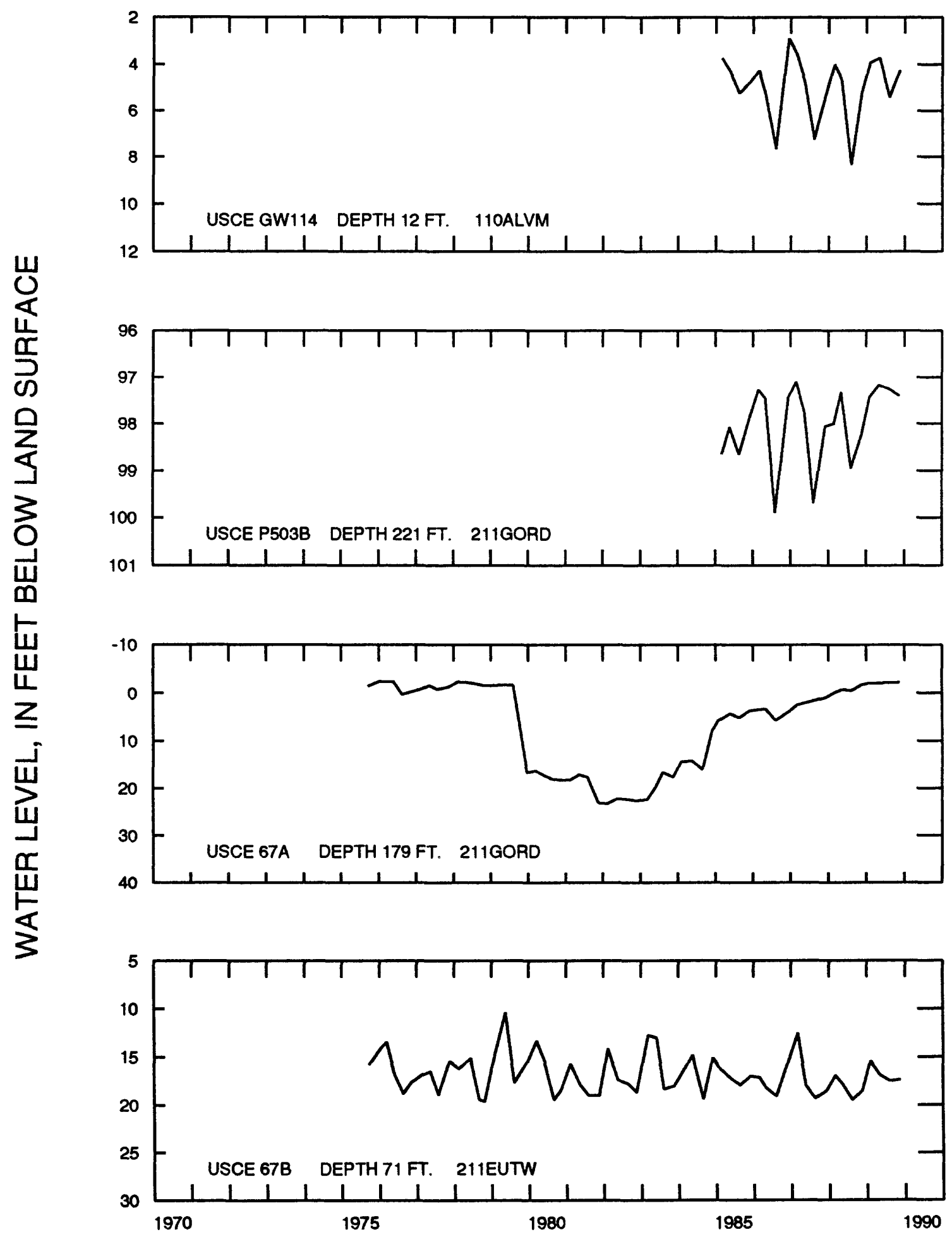

HYDROGRAPHS OF TENNESSEE-TOMBIGBEE OBSERVATION WELLS 


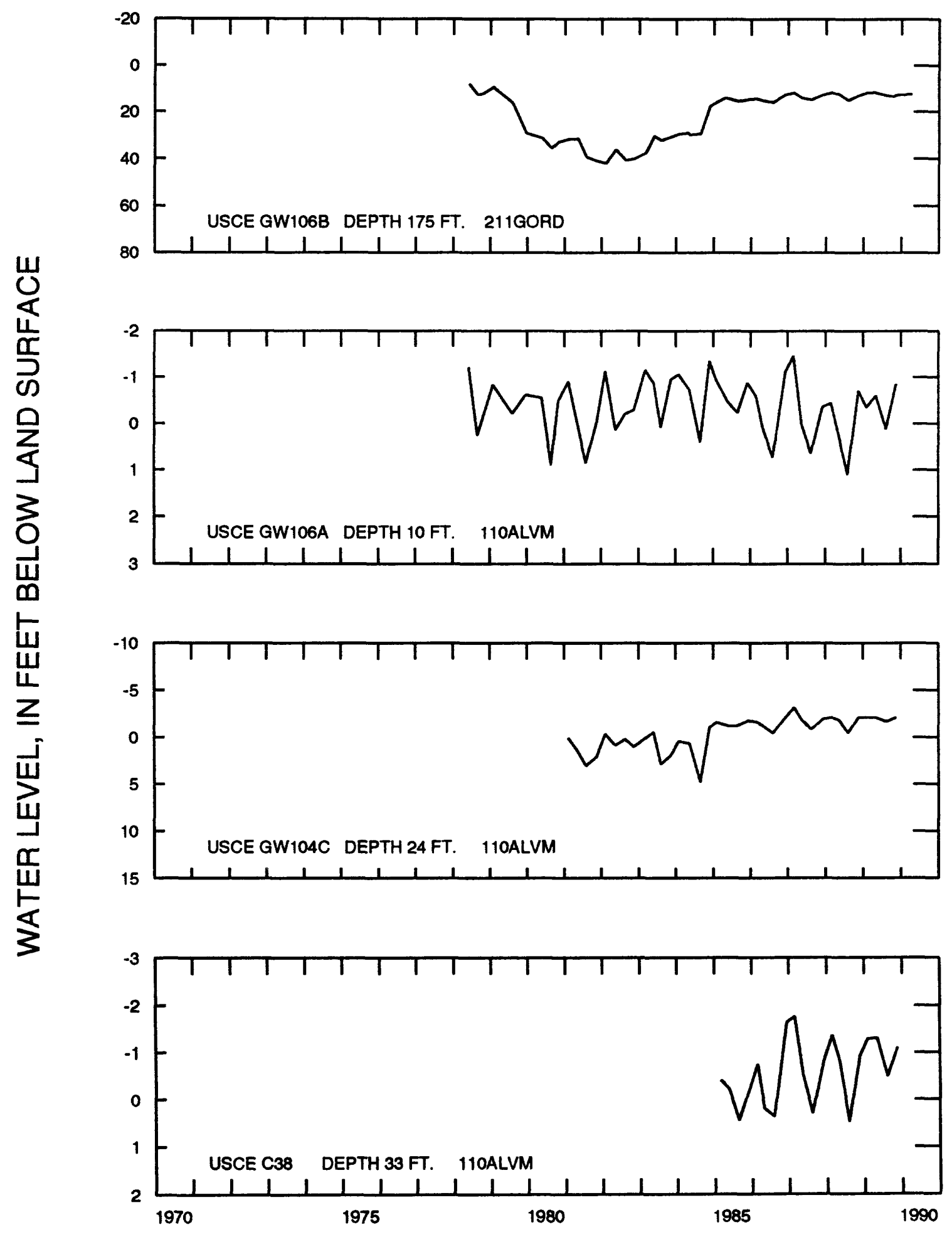

HYDROGRAPHS OF TENNESSEE-TOMBIGBEE OBSERVATION WELLS 


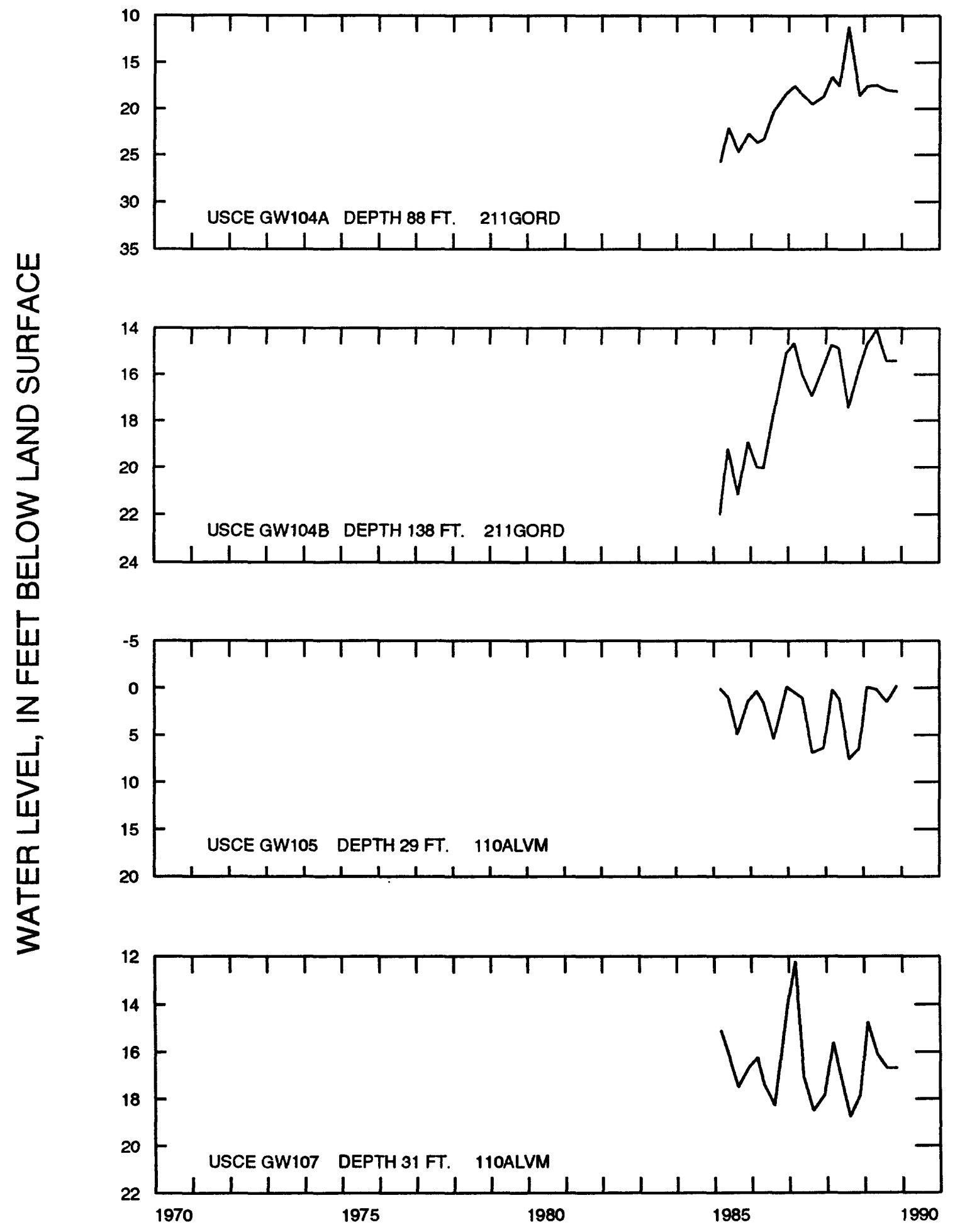

HYDROGRAPHS OF TENNESSEE-TOMBIGBEE OBSERVATION WELLS 


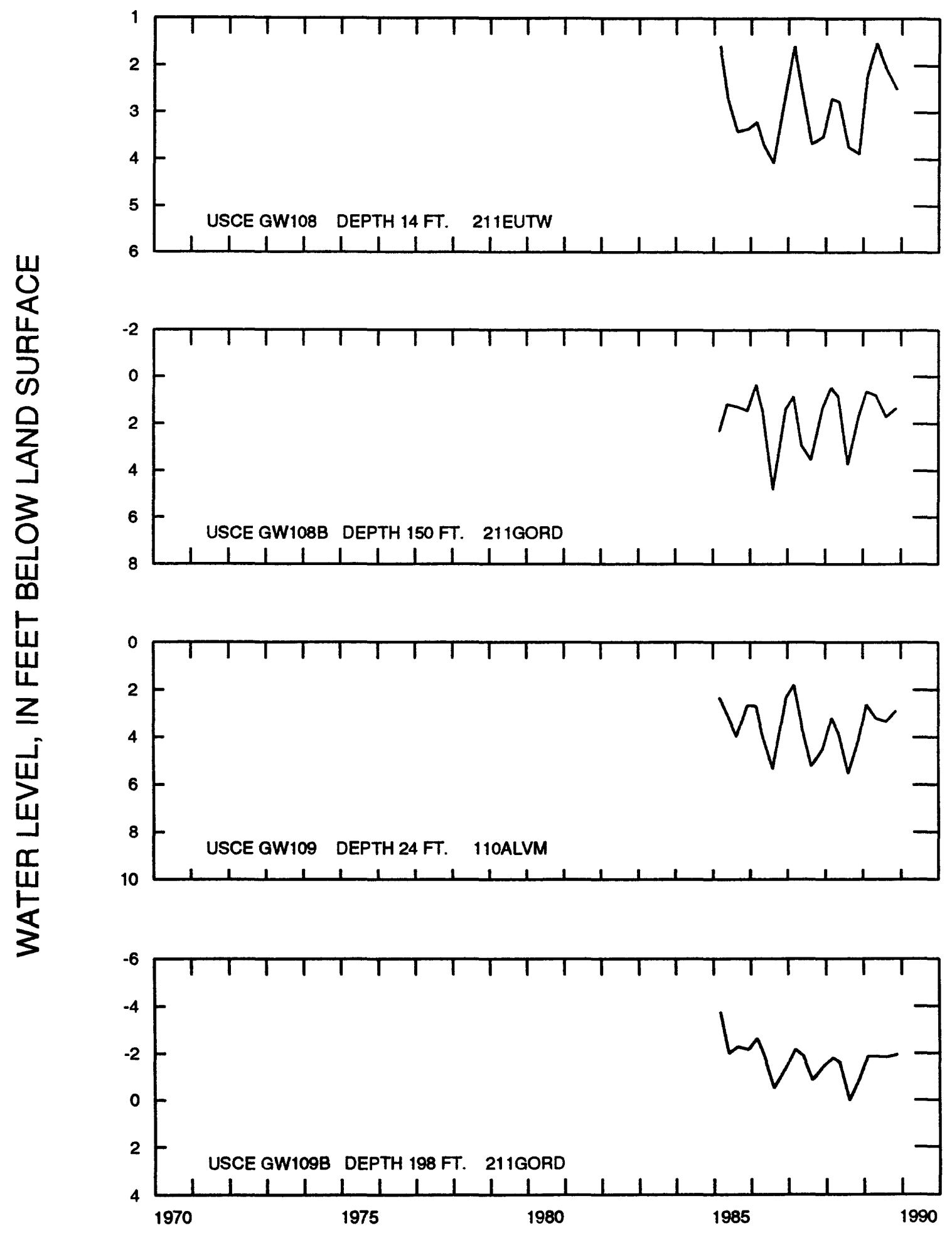

HYDROGRAPHS OF TENNESSEE-TOMBIGBEE OBSERVATION WELLS 


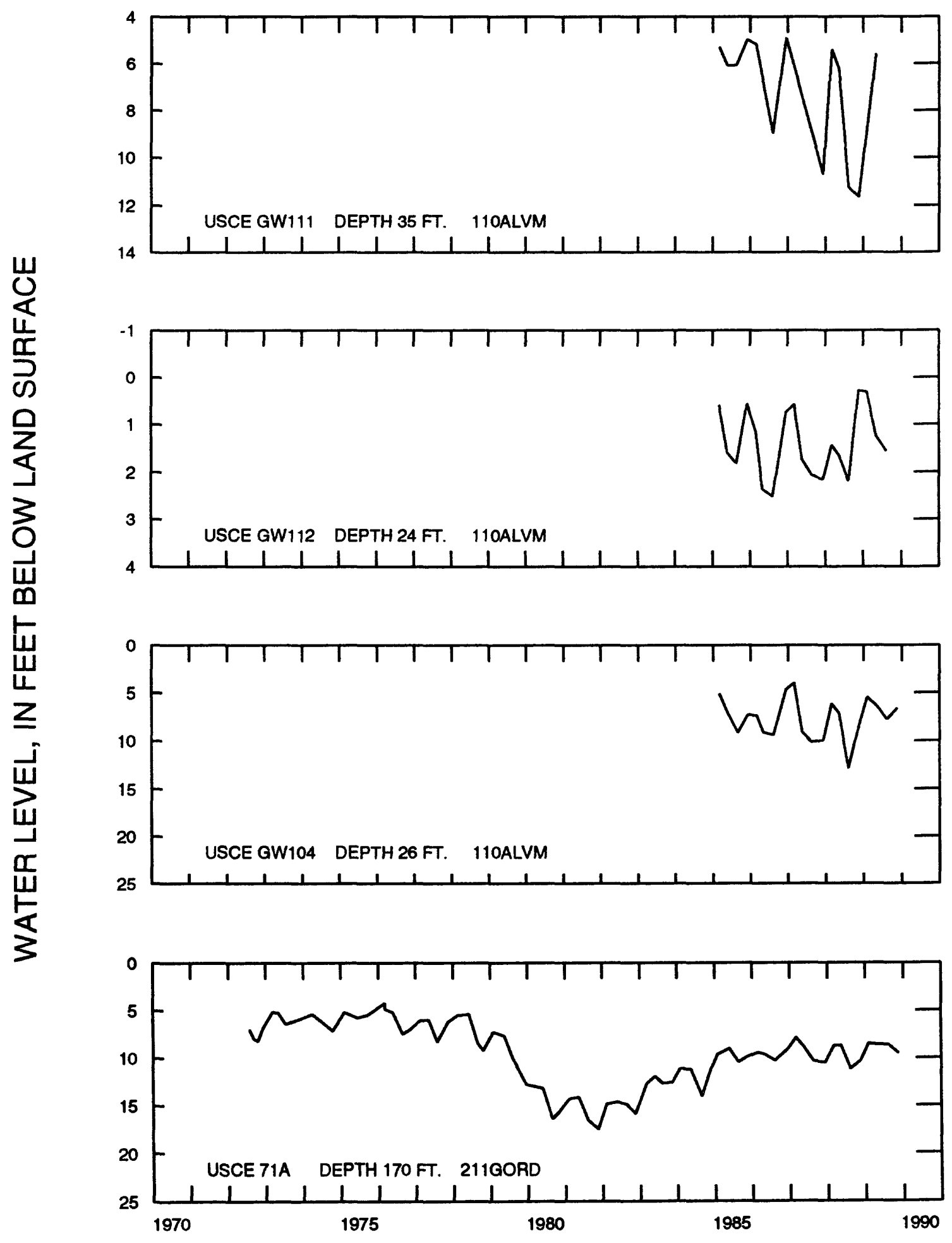

HYDROGRAPHS OF TENNESSEE-TOMBIGBEE OBSERVATION WELLS 


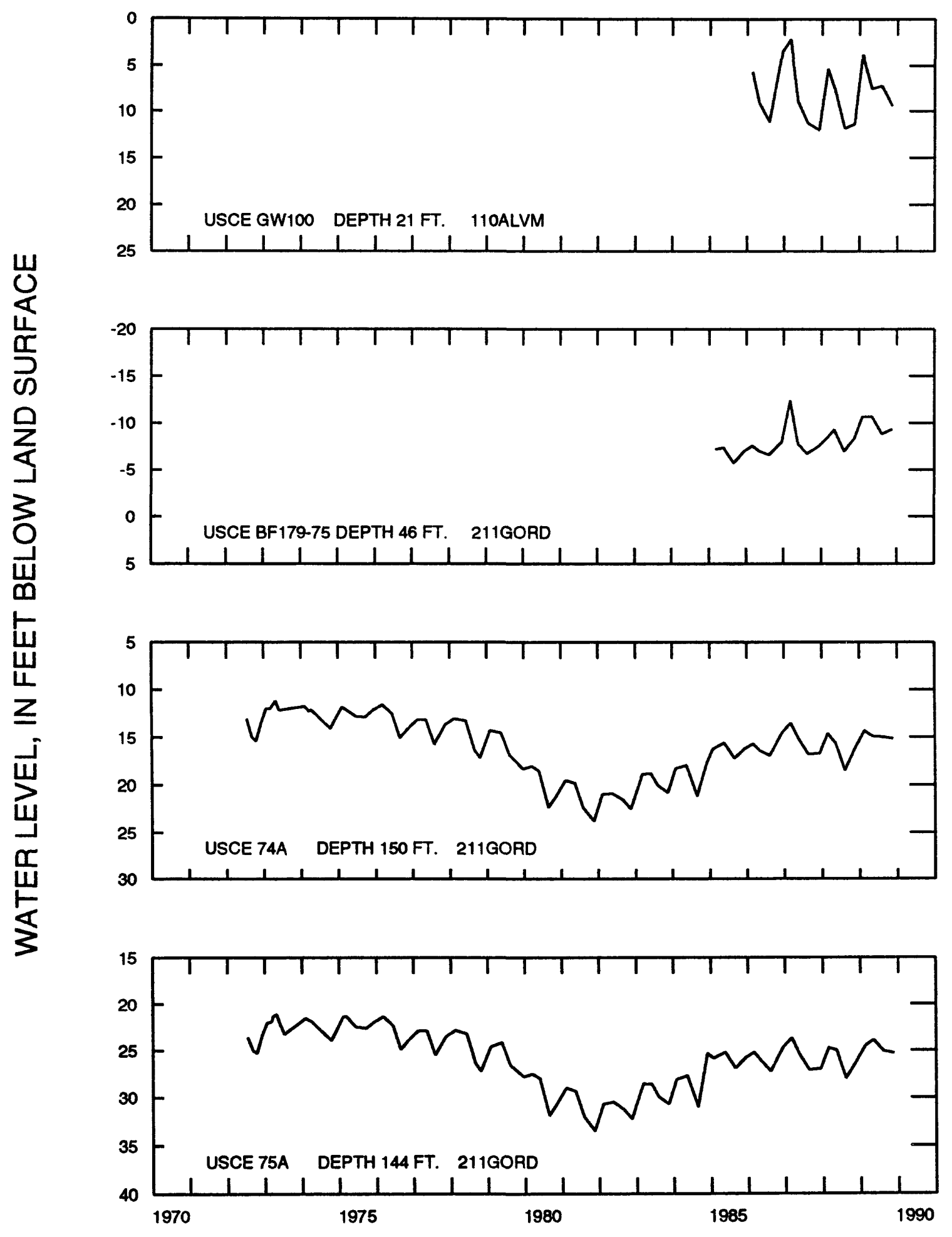

HYDROGRAPHS OF TENNESSEE-TOMBIGBEE OBSERVATION WELLS 


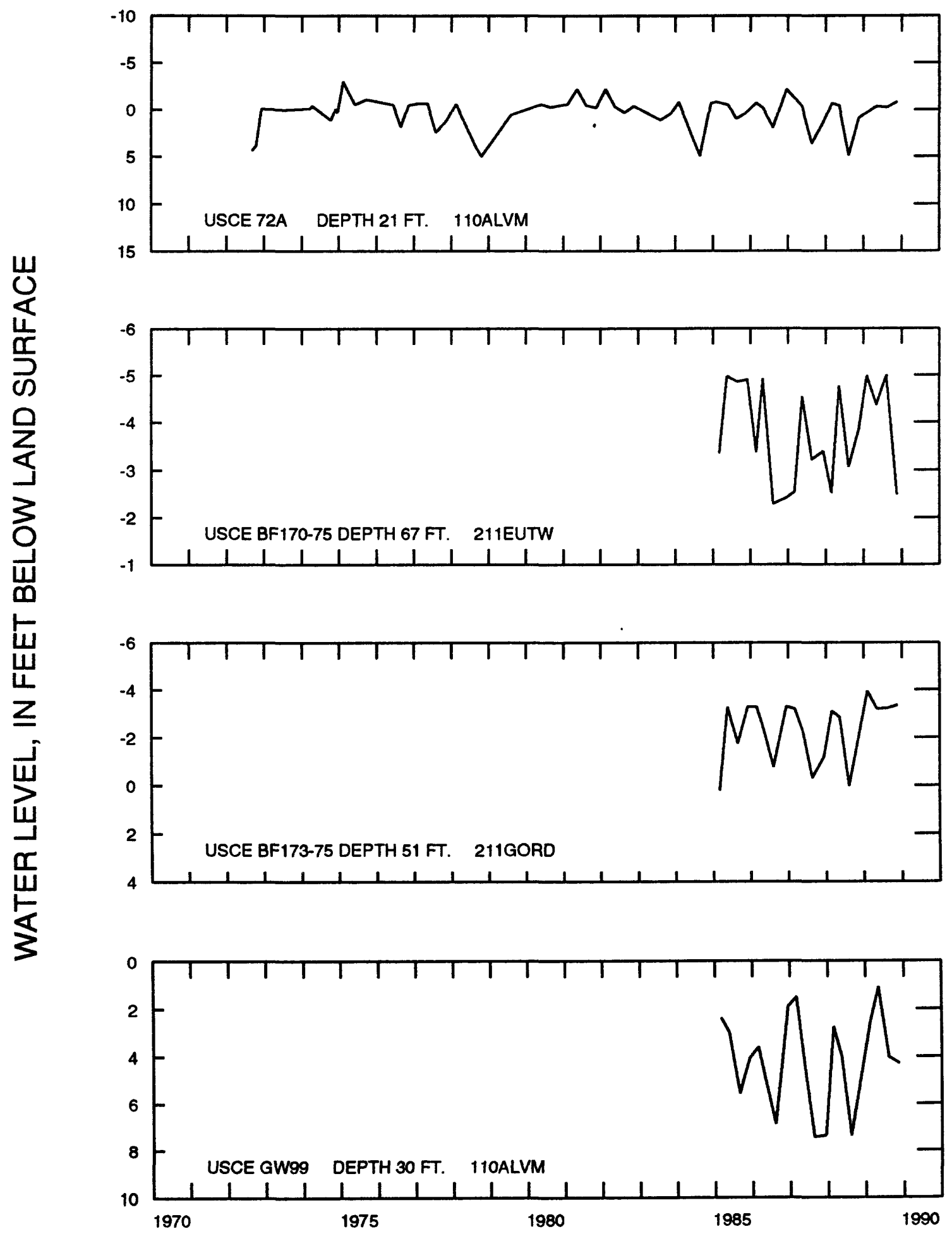

HYDROGRAPHS OF TENNESSEE-TOMBIGBEE OBSERVATION WELLS 


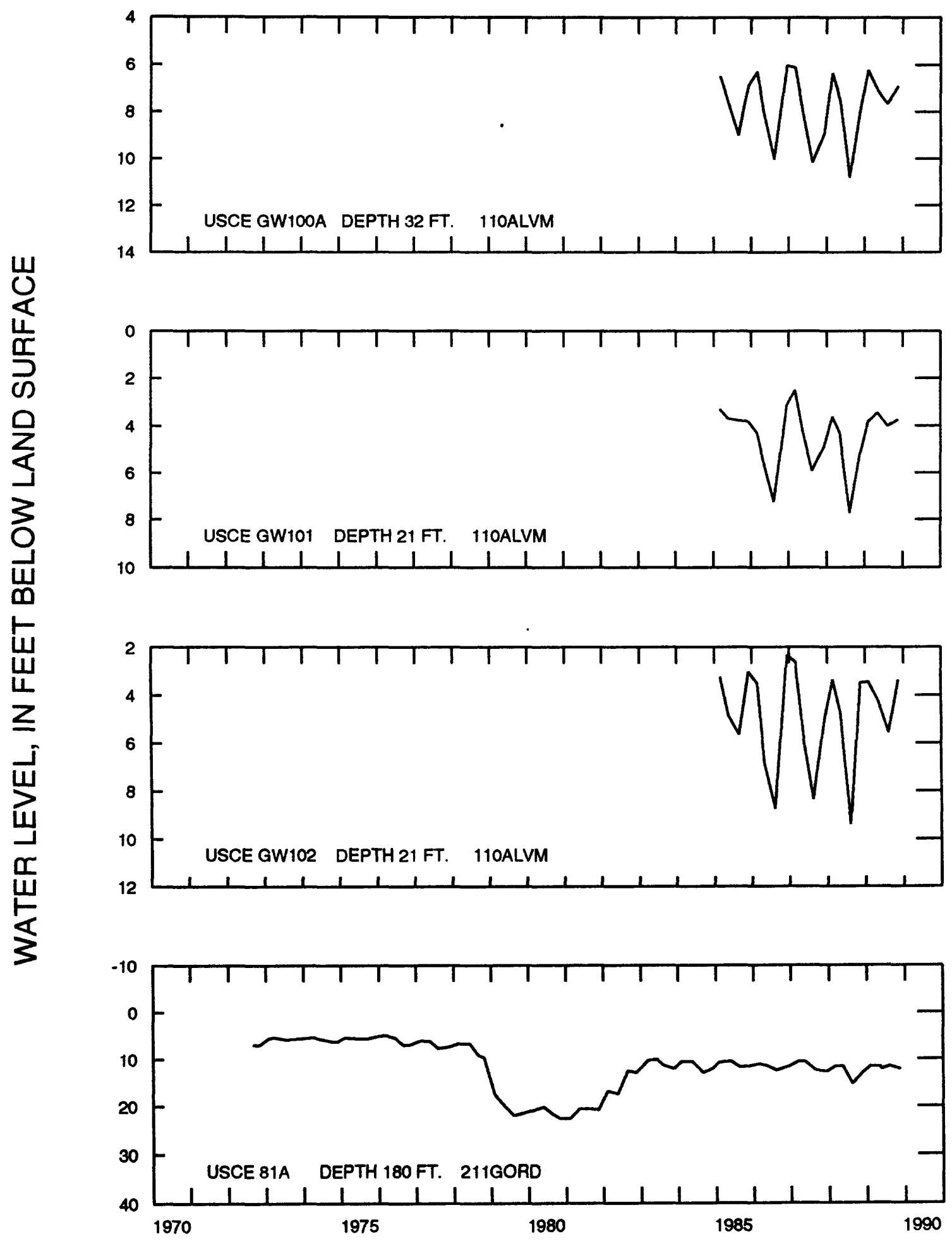

HYDROGRAPHS OF TENNESSEE-TOMBIGBEE OBSERVATION WELLS 


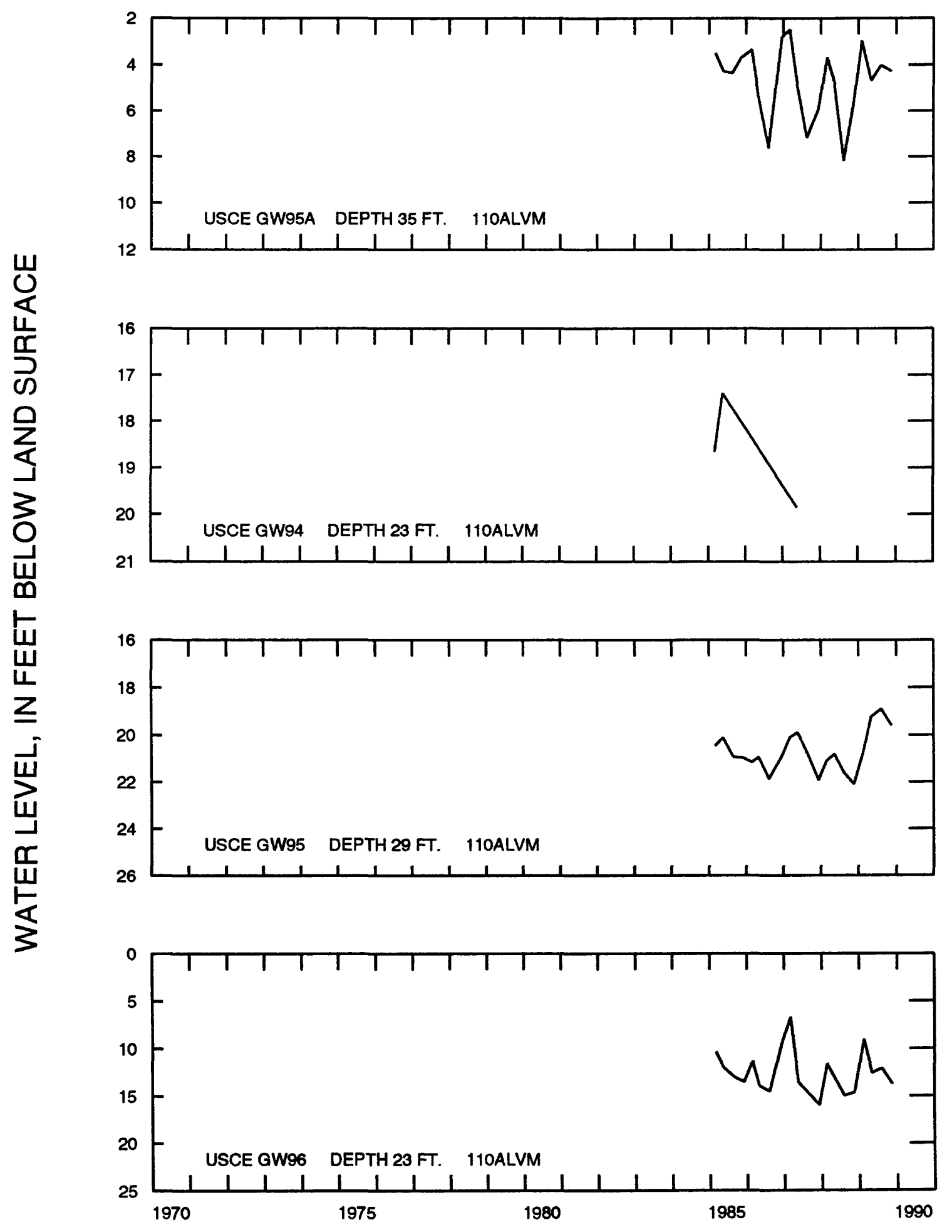

HYDROGRAPHS OF TENNESSEE-TOMBIGBEE OBSERVATION WELLS 


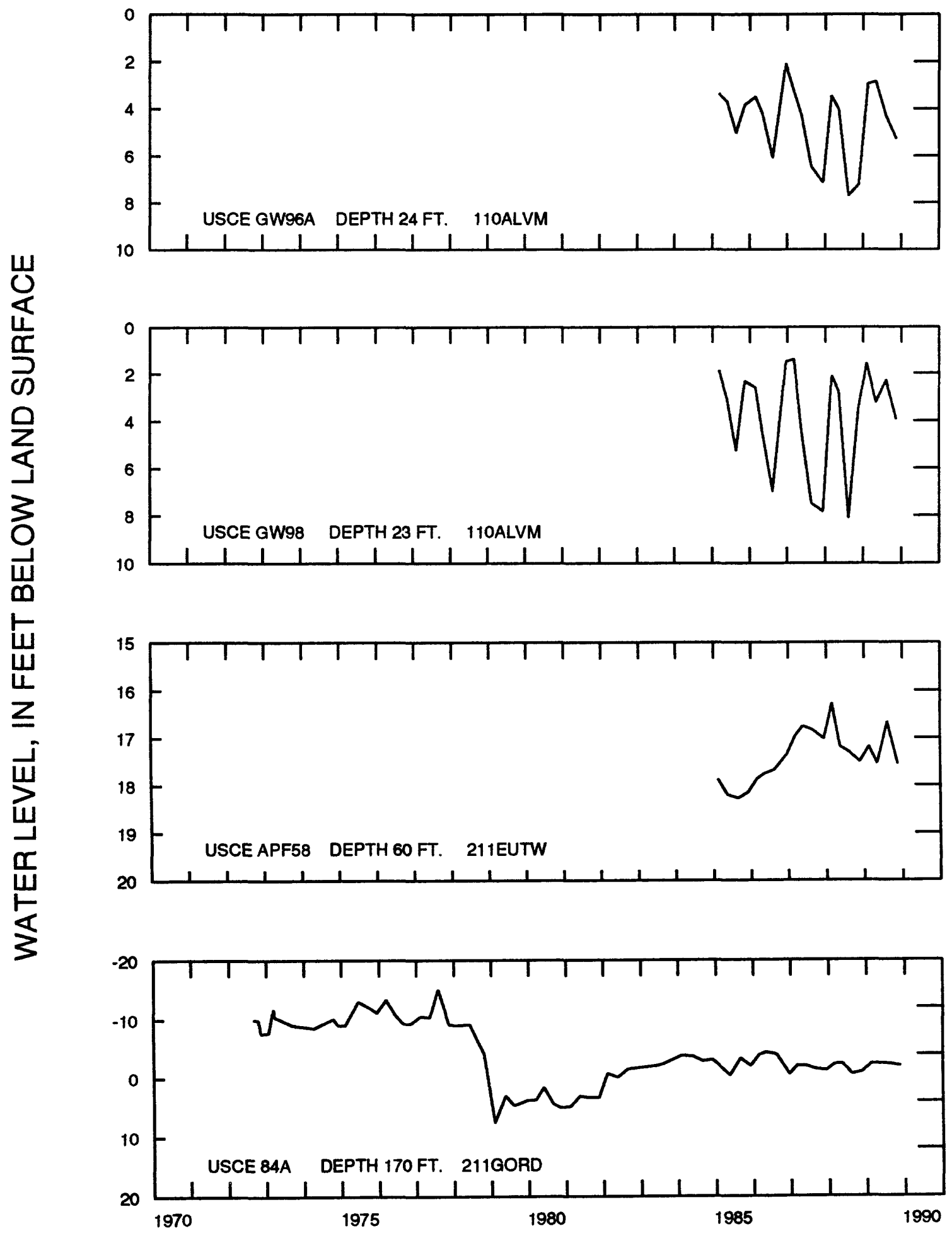

HYDROGRAPHS OF TENNESSEE-TOMBIGBEE OBSERVATION WELLS 


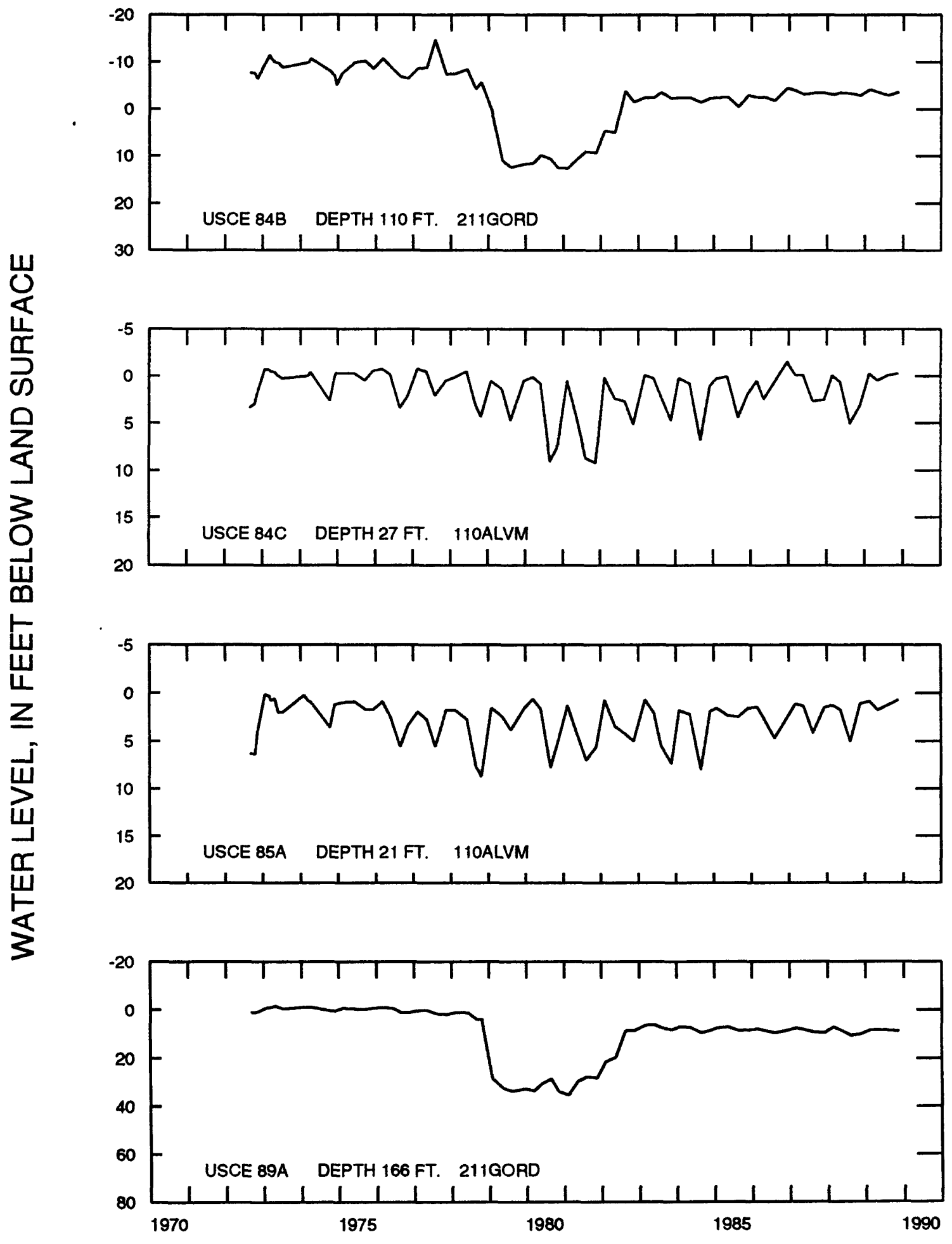

HYDROGRAPHS OF TENNESSEE-TOMBIGBEE OBSERVATION WELLS 


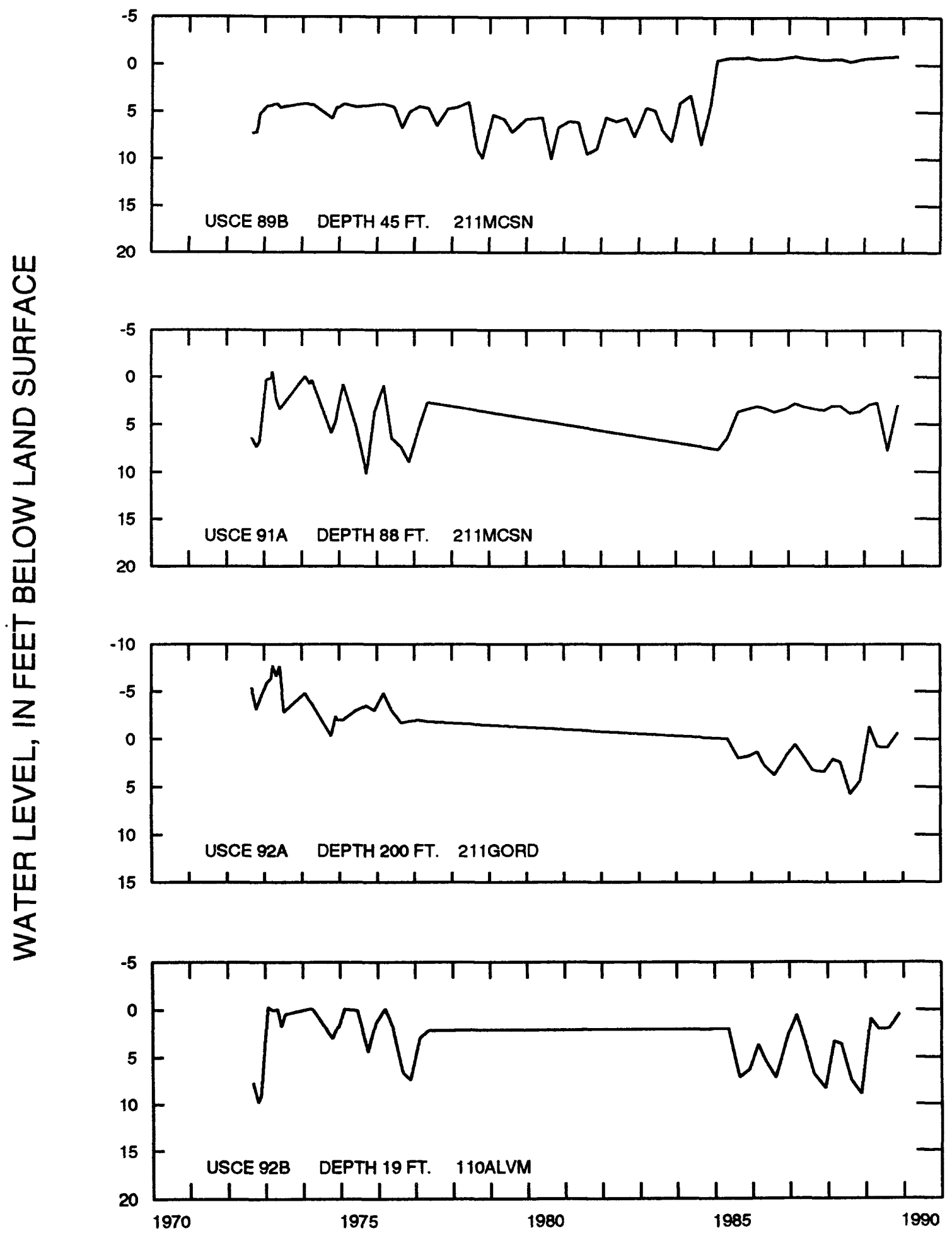

HYDROGRAPHS OF TENNESSEE-TOMBIGBEE OBSERVATION WELLS 


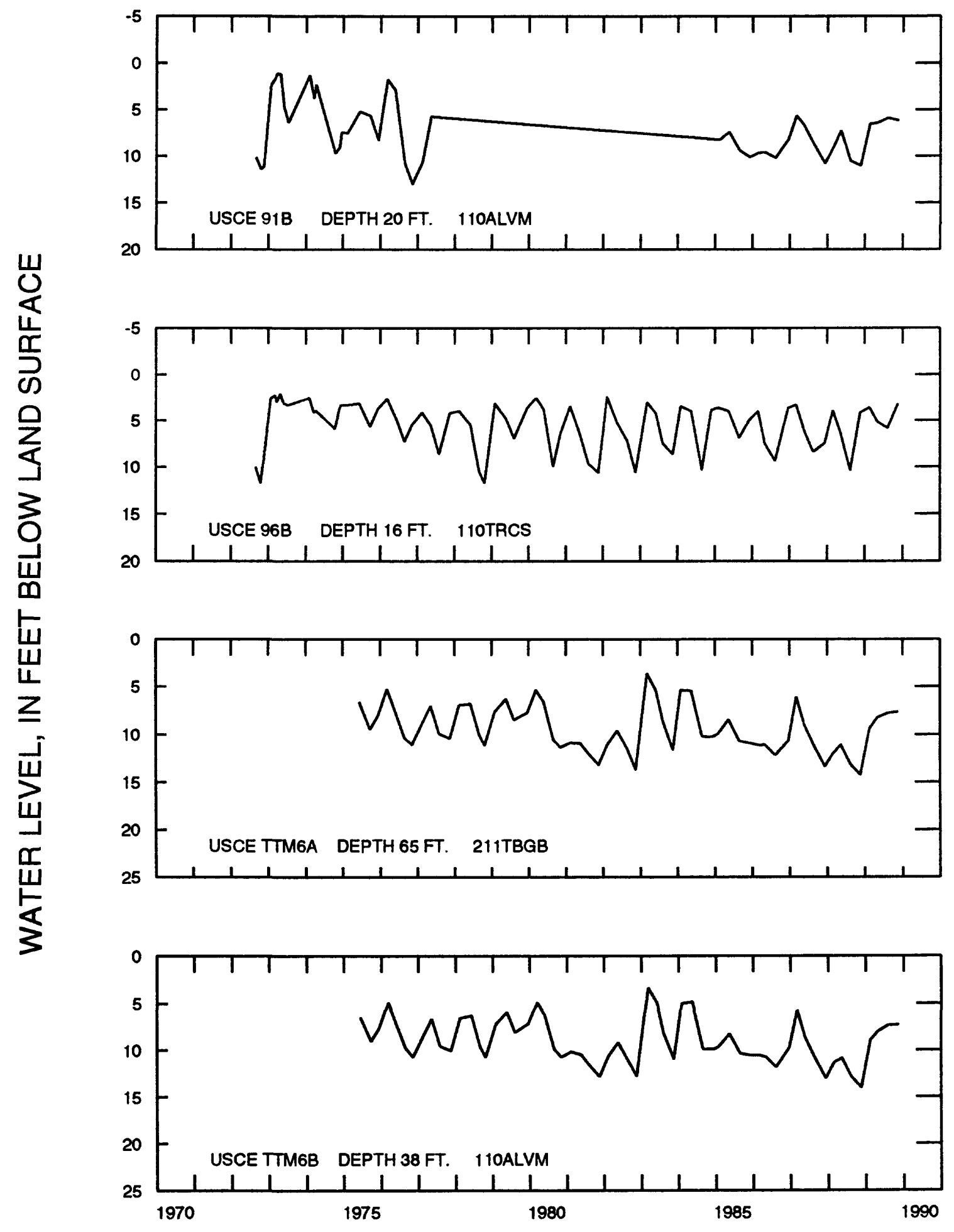

HYDROGRAPHS OF TENNESSEE-TOMBIGBEE OBSERVATION WELLS 


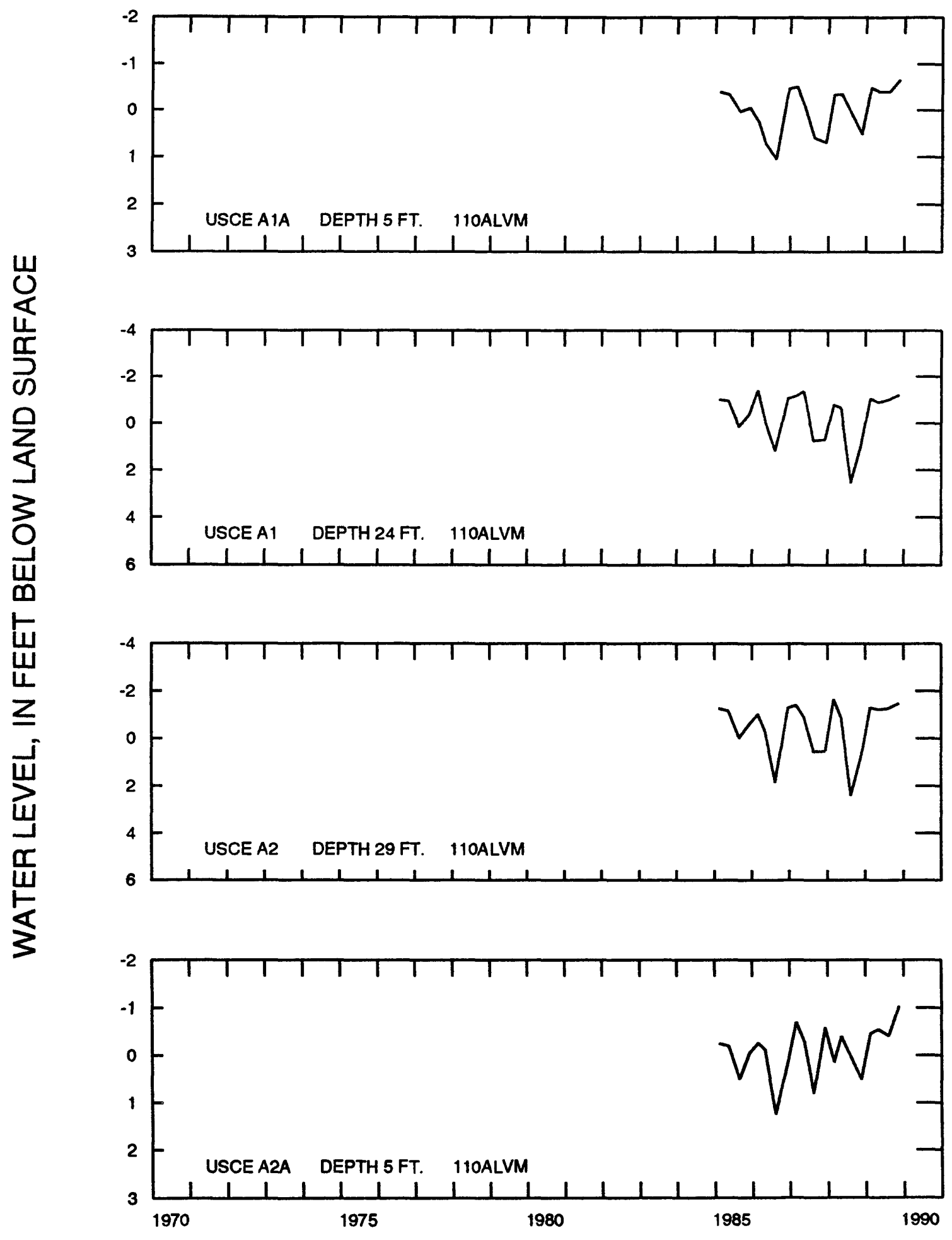

HYDROGRAPHS OF TENNESSEE-TOMBIGBEE OBSERVATION WELLS 


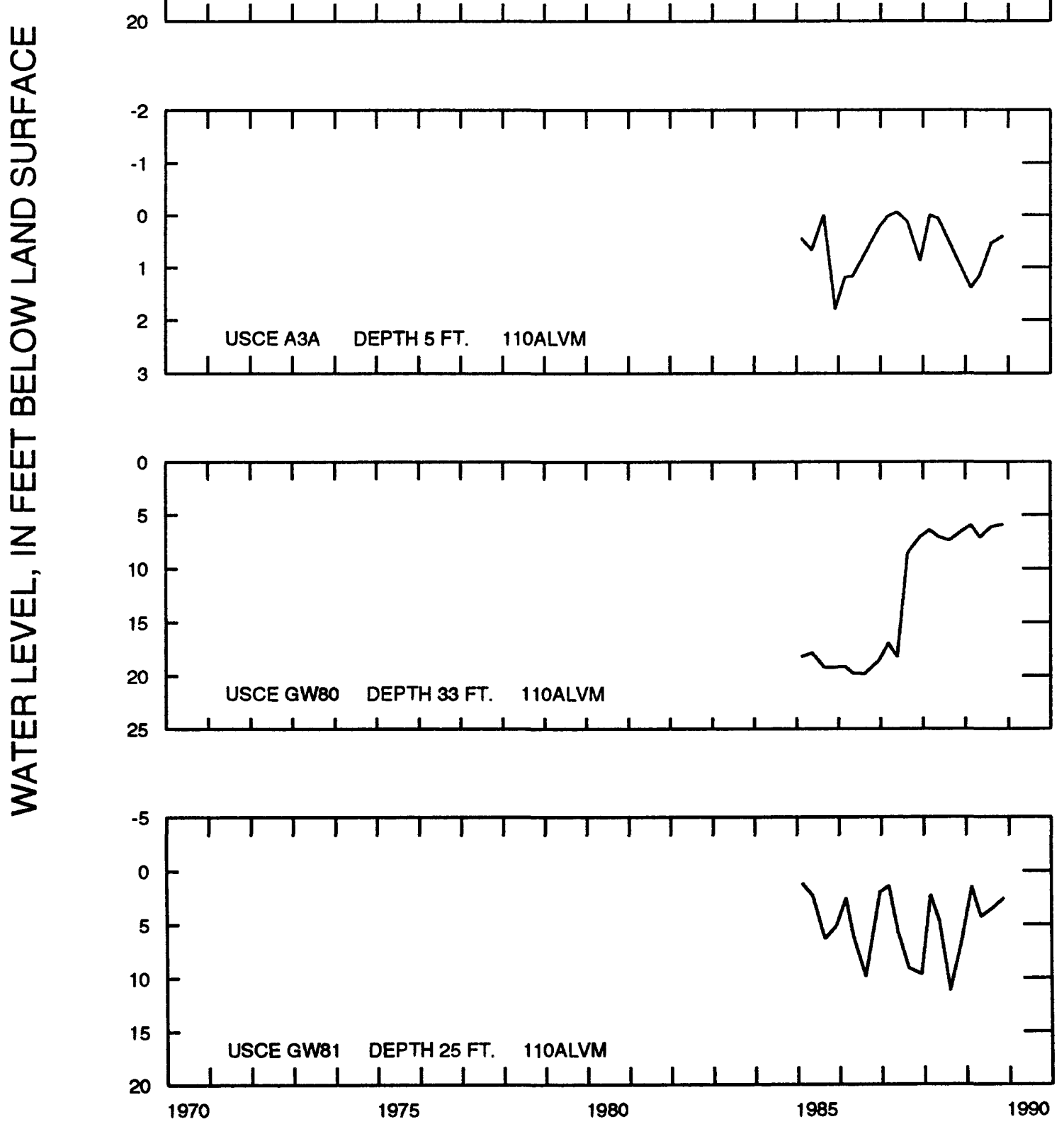

HYDROGRAPHS OF TENNESSEE-TOMBIGBEE OBSERVATION WELLS 


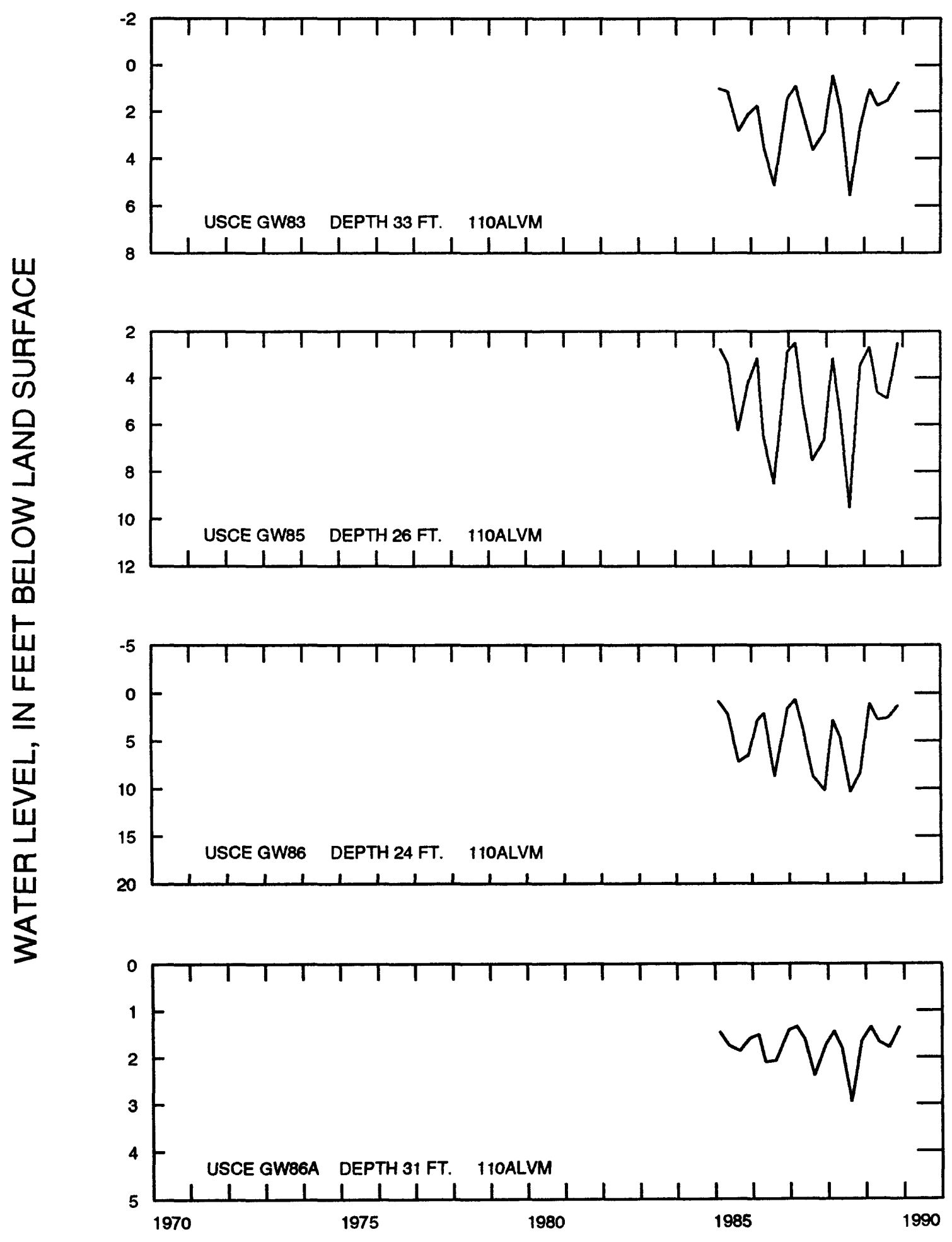

HYDROGRAPHS OF TENNESSEE-TOMBIGBEE OBSERVATION WELLS 


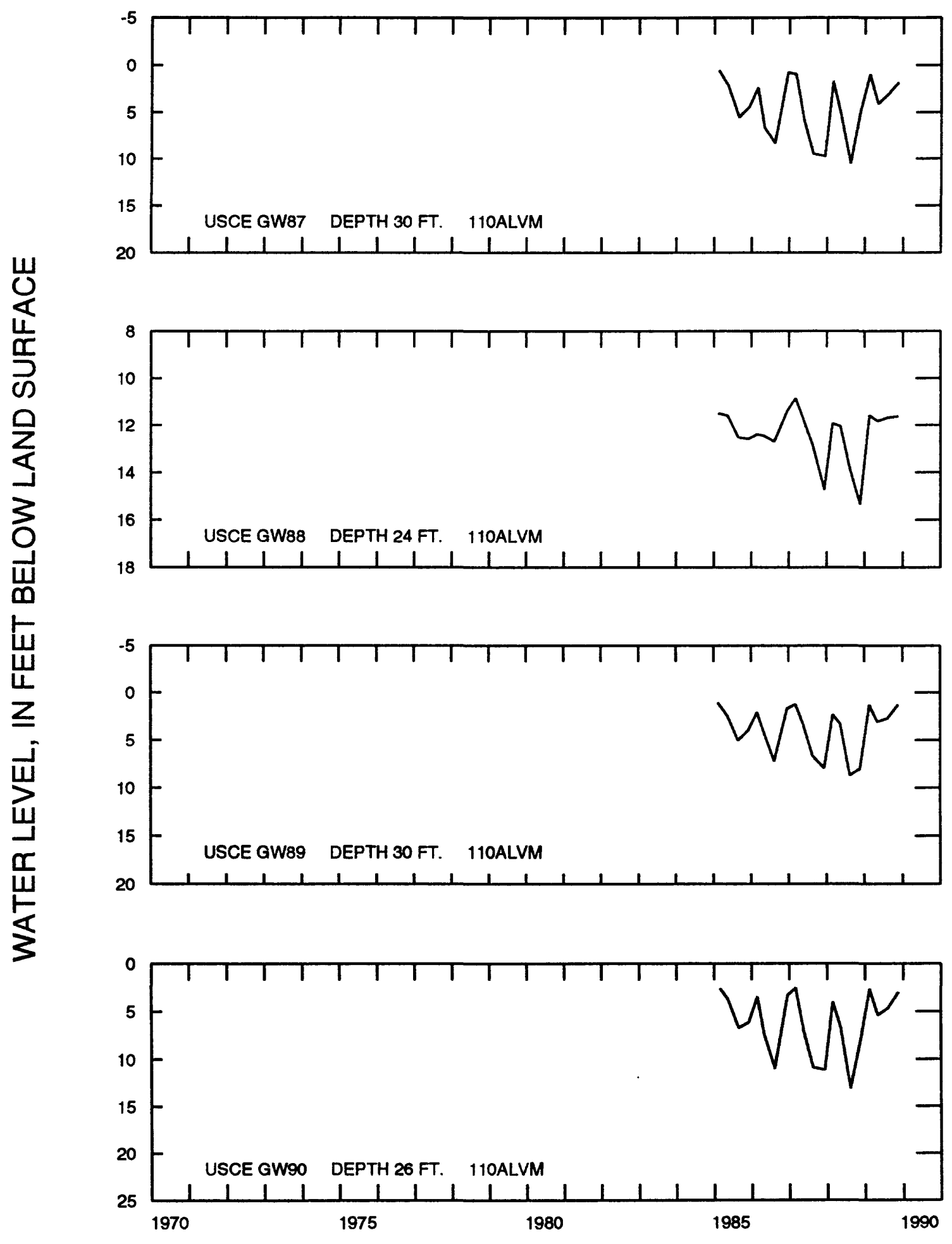

HYDROGRAPHS OF TENNESSEE-TOMBIGBEE OBSERVATION WELLS 


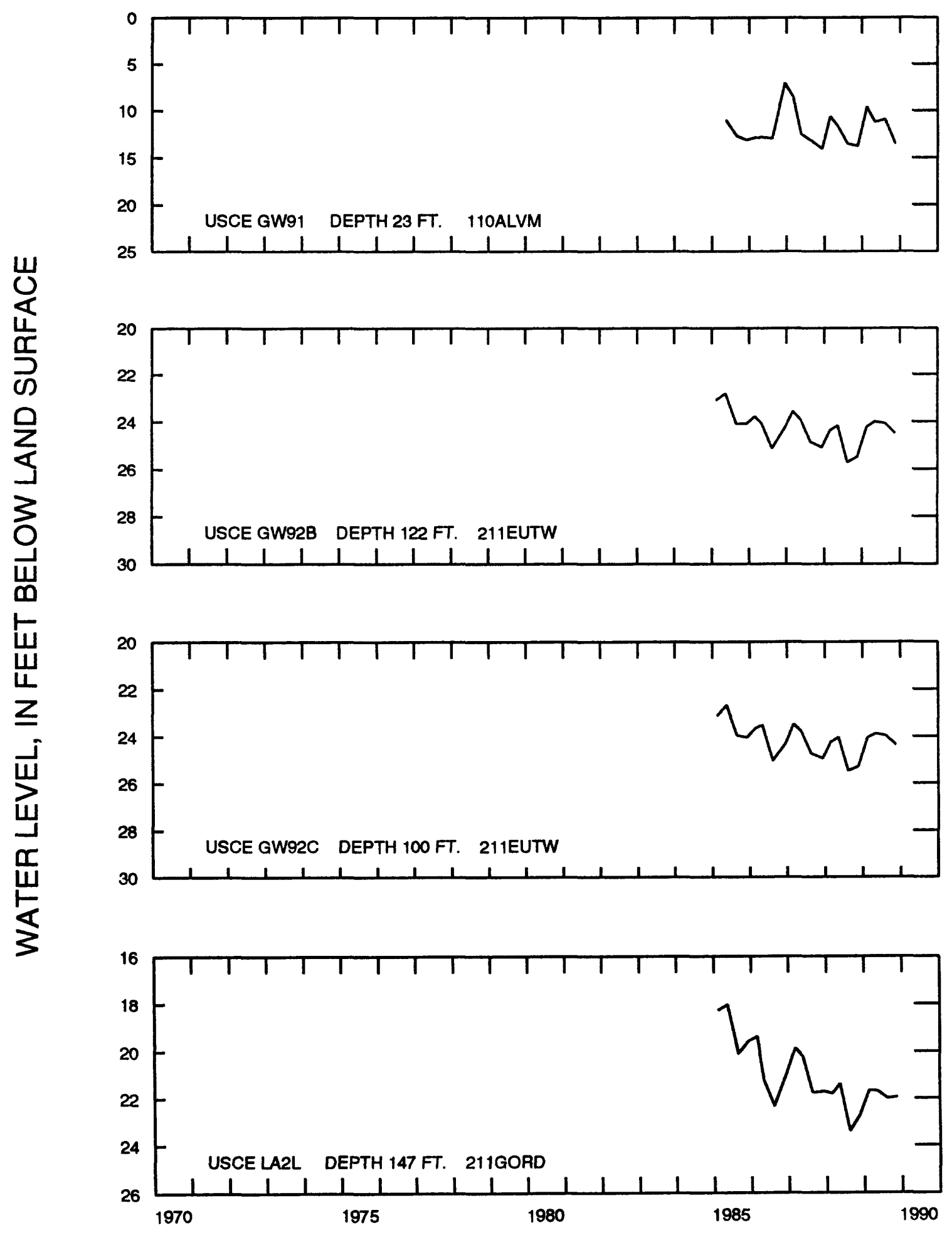

HYDROGRAPHS OF TENNESSEE-TOMBIGBEE OBSERVATION WELLS 


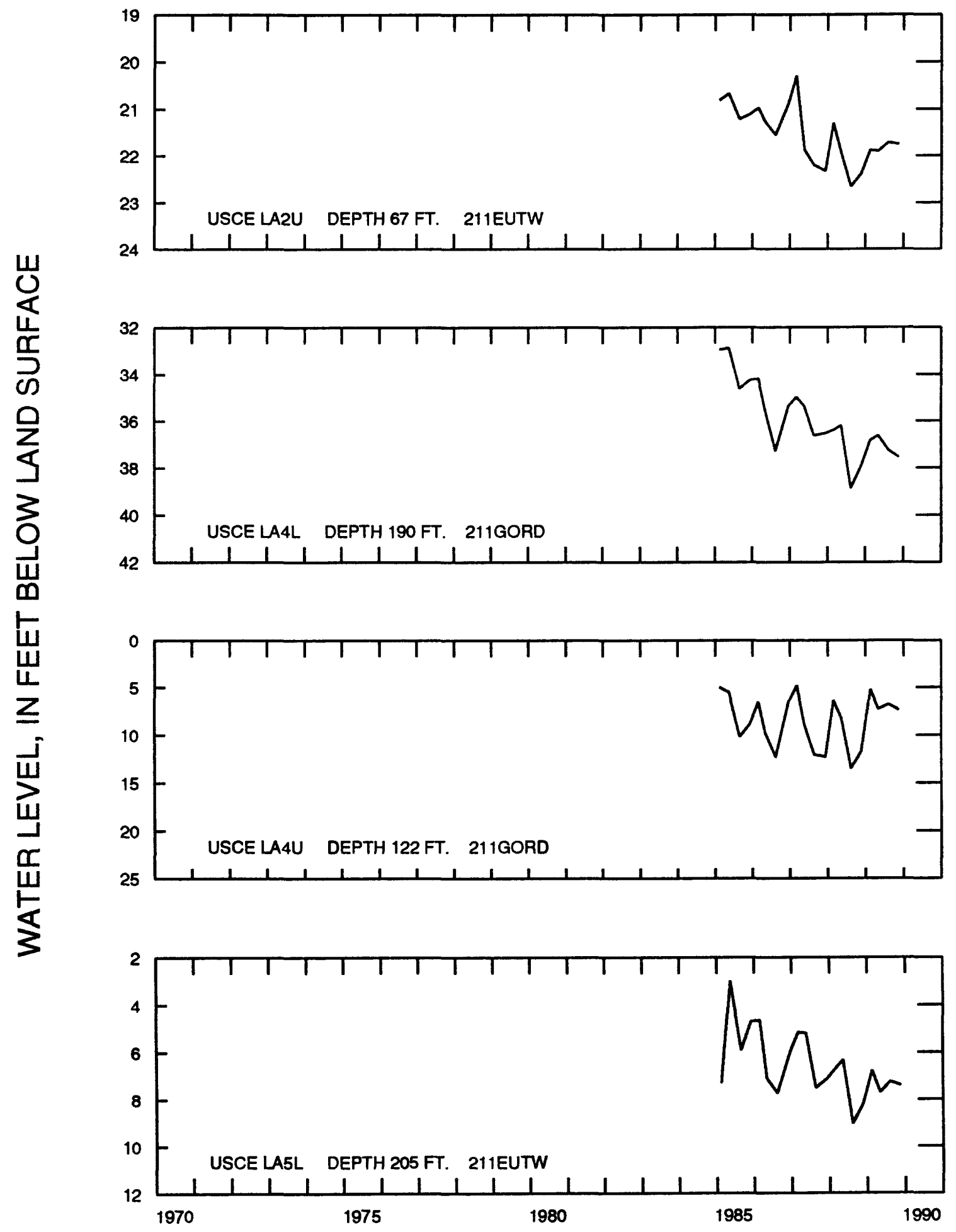

HYDROGRAPHS OF TENNESSEE-TOMBIGBEE OBSERVATION WELLS 


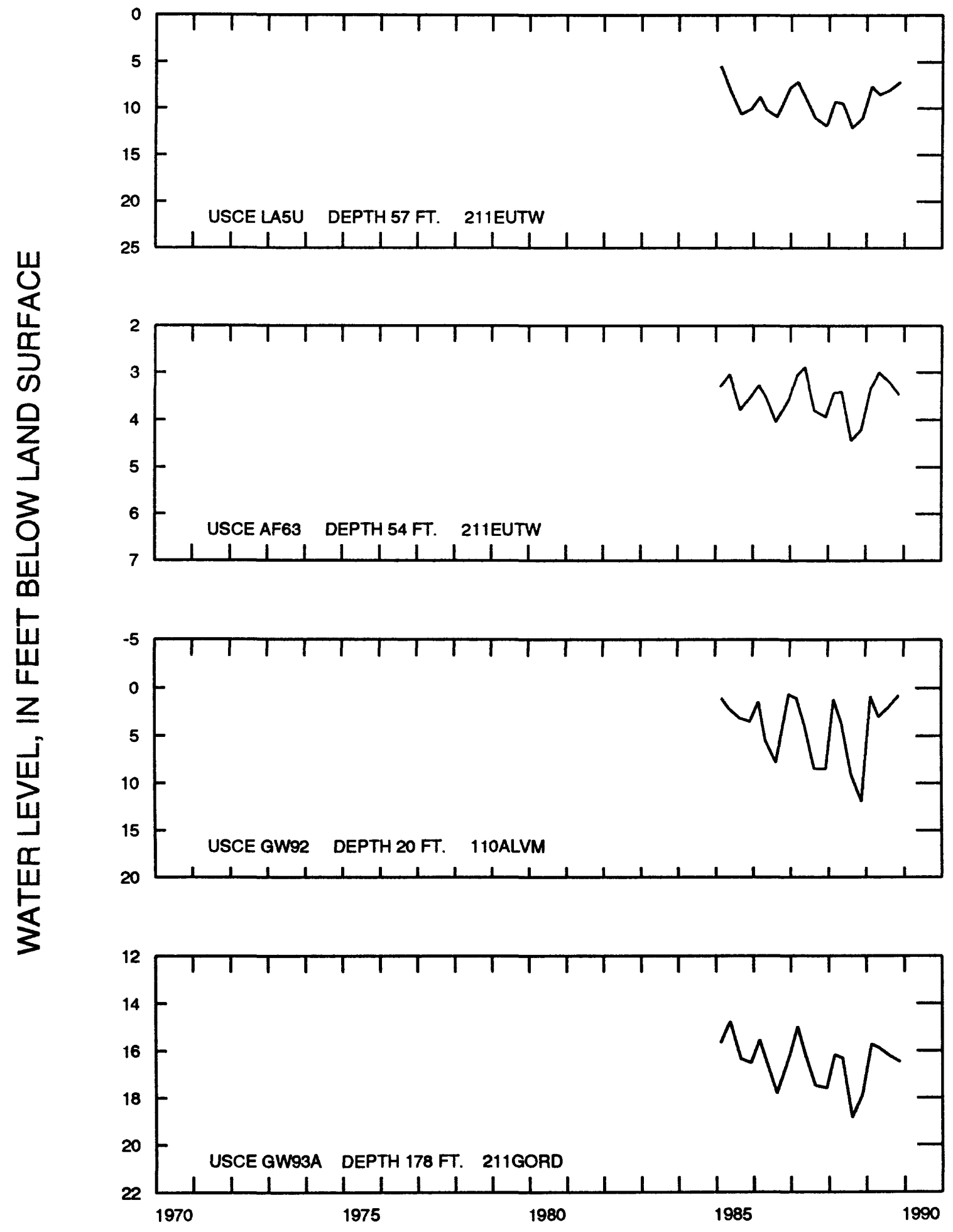

HYDROGRAPHS OF TENNESSEE-TOMBIGBEE OBSERVATION WELLS 


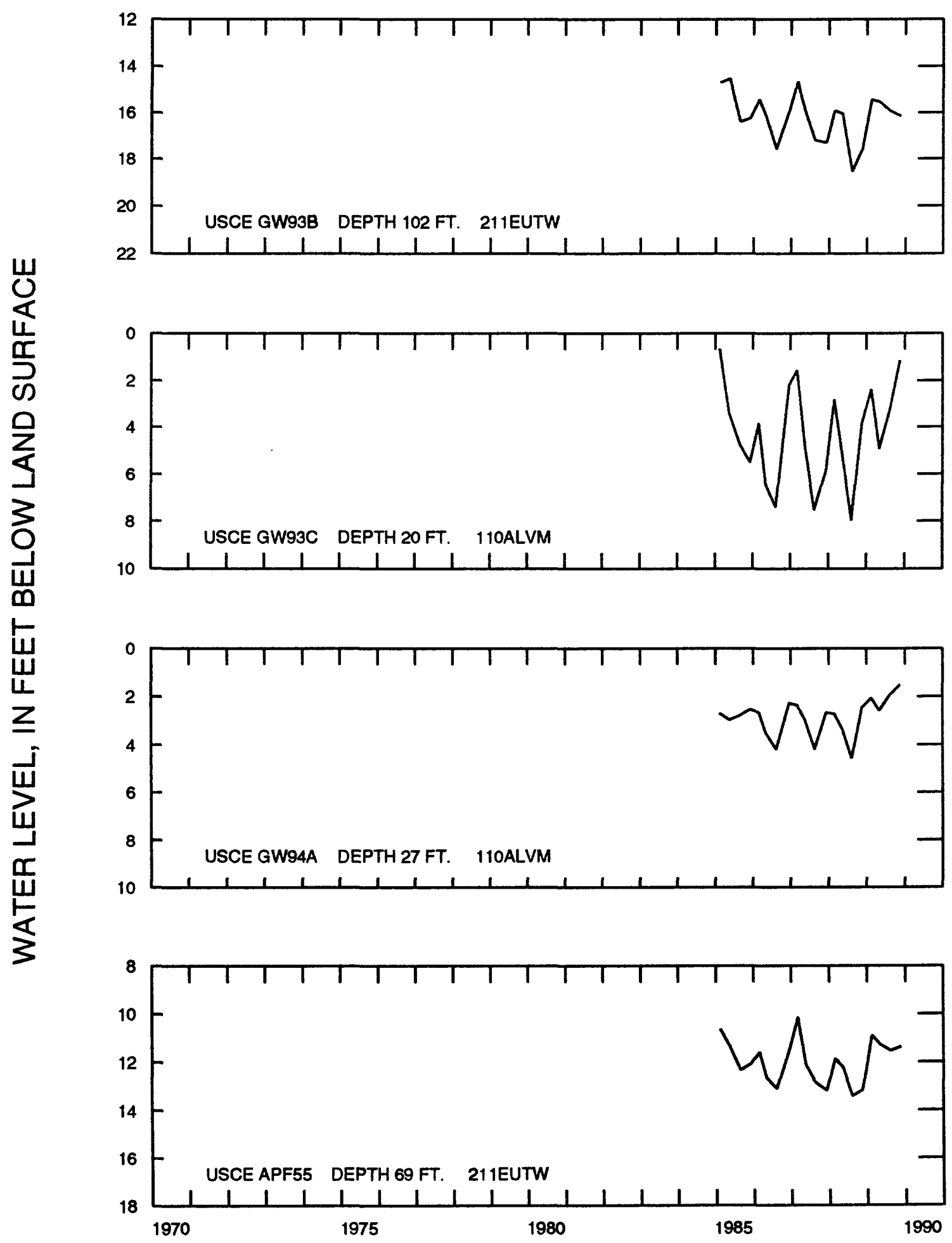

HYDROGRAPHS OF TENNESSEE-TOMBIGBEE OBSERVATION WELLS 


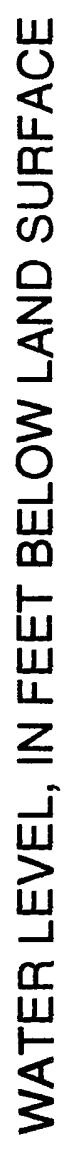
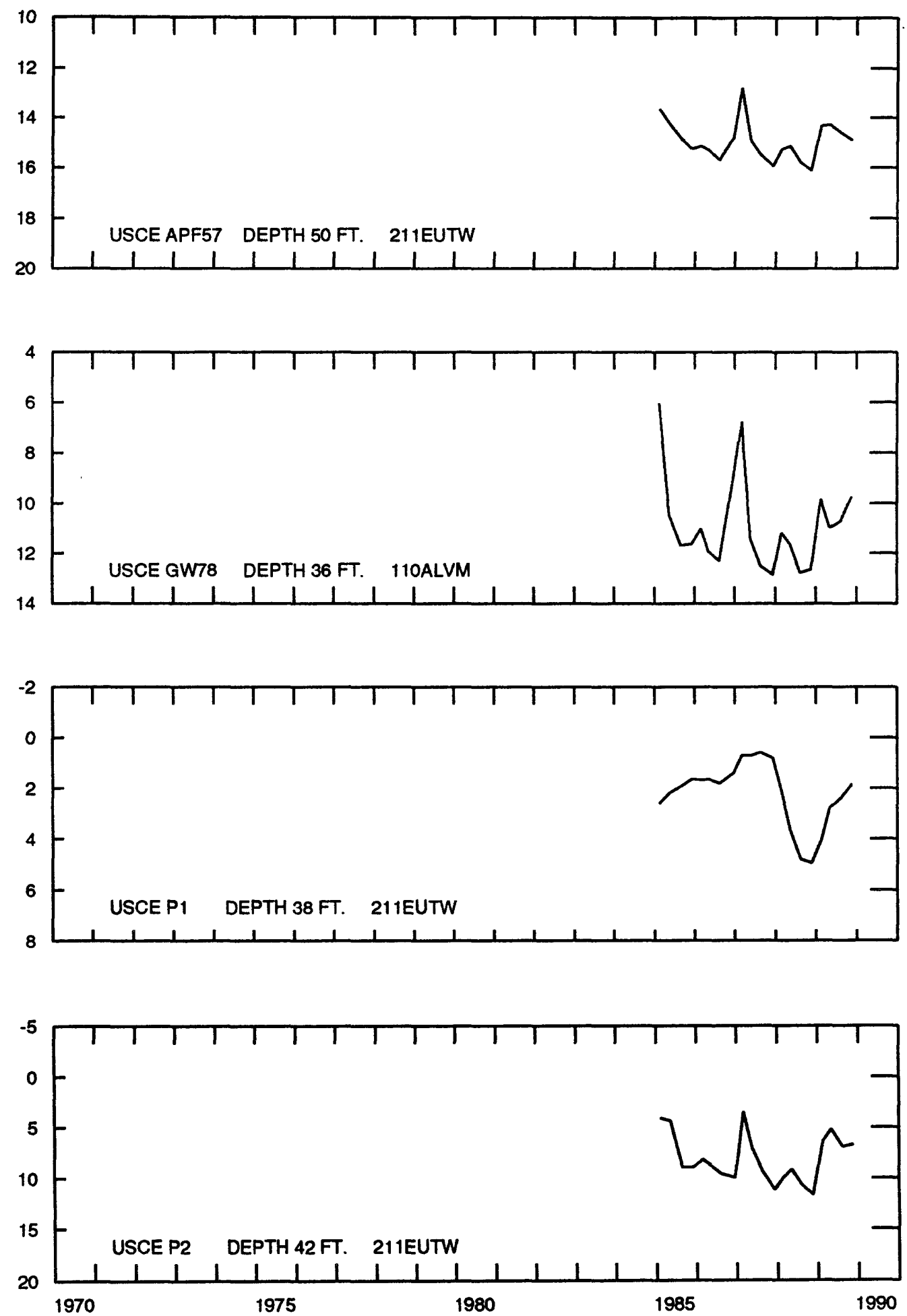

HYDROGRAPHS OF TENNESSEE-TOMBIGBEE OBSERVATION WELLS 


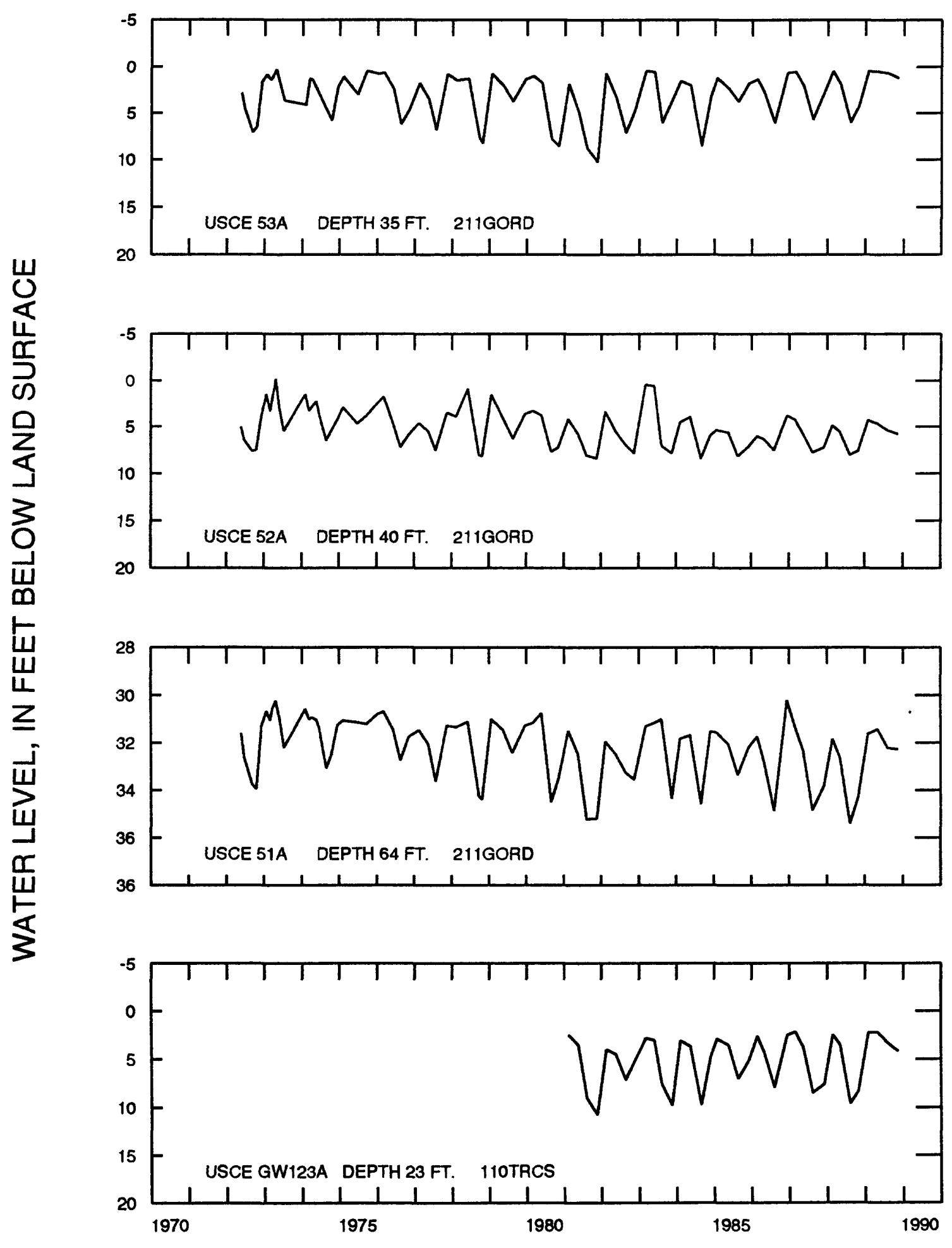

HYDROGRAPHS OF TENNESSEE-TOMBIGBEE OBSERVATION WELLS 


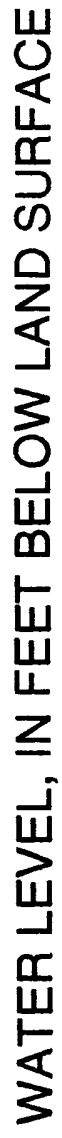
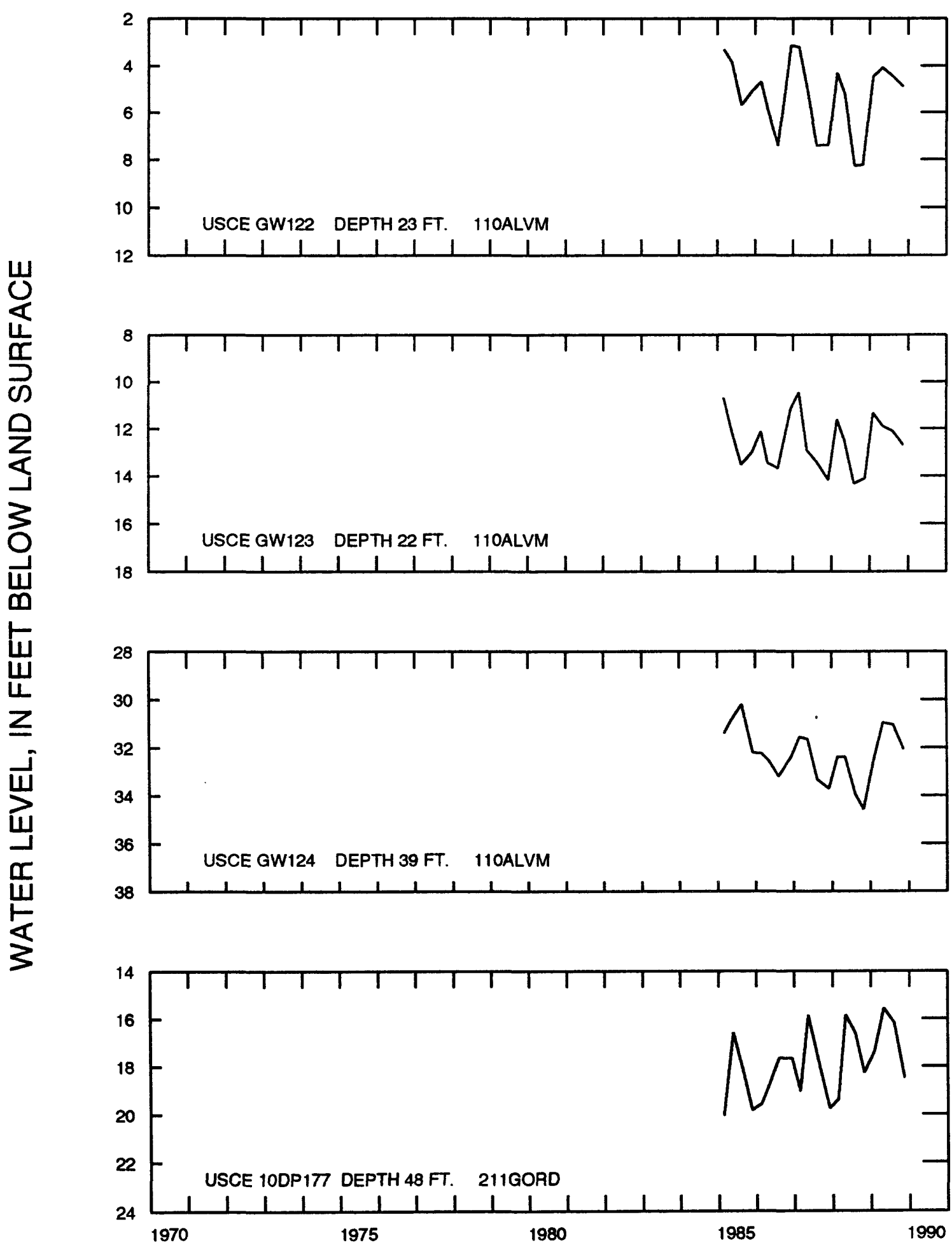

HYDROGRAPHS OF TENNESSEE-TOMBIGBEE OBSERVATION WELLS 


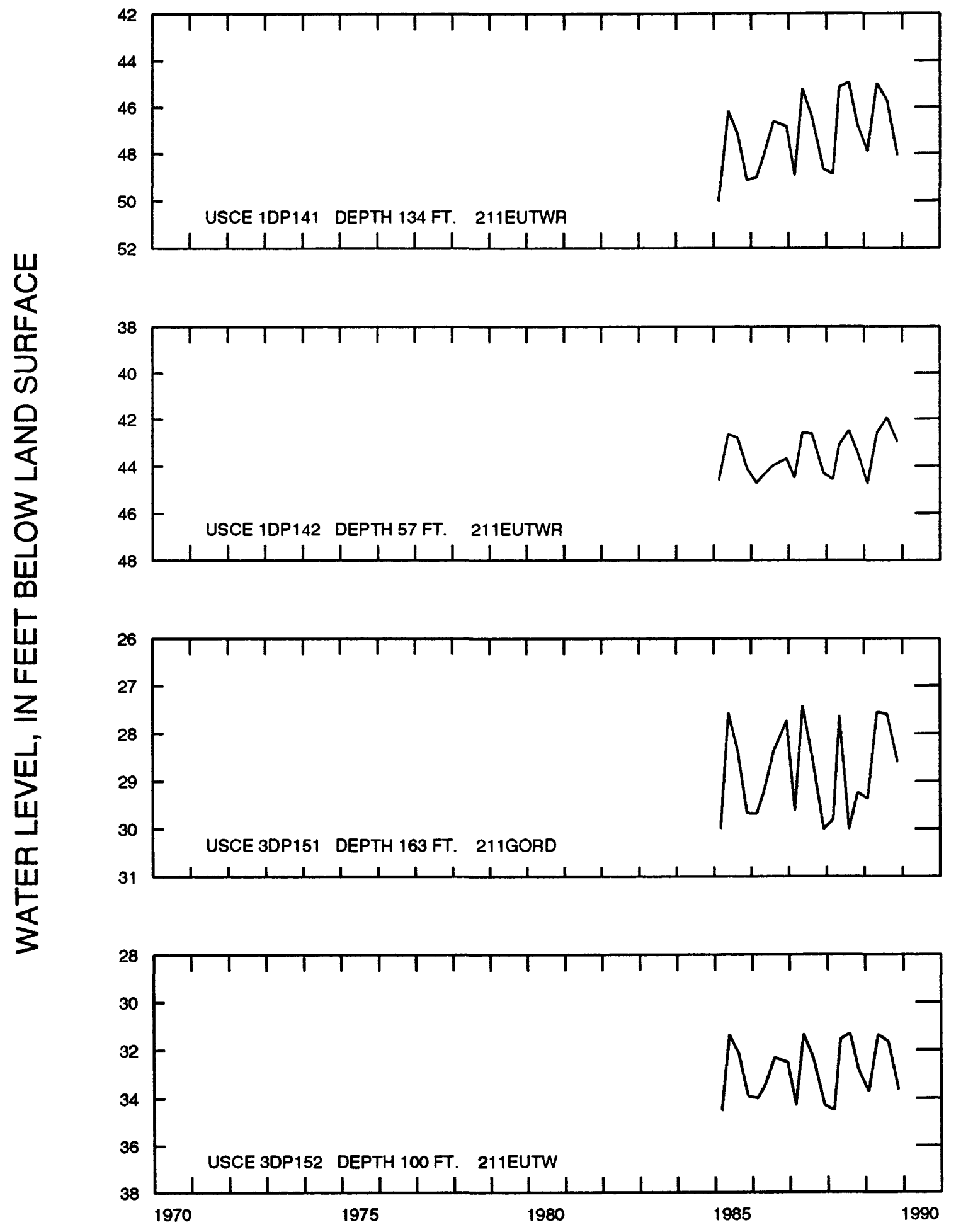

HYDROGRAPHS OF TENNESSEE-TOMBIGBEE OBSERVATION WELLS 


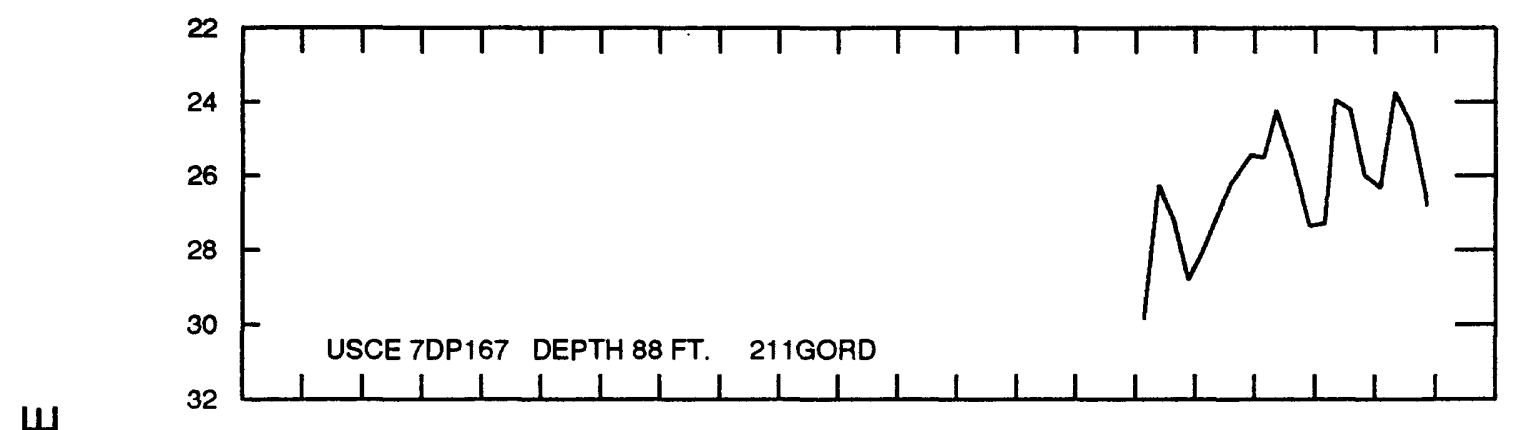

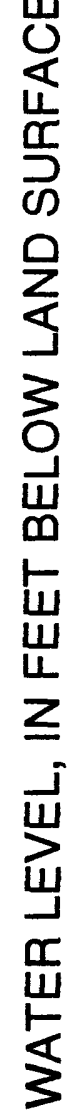

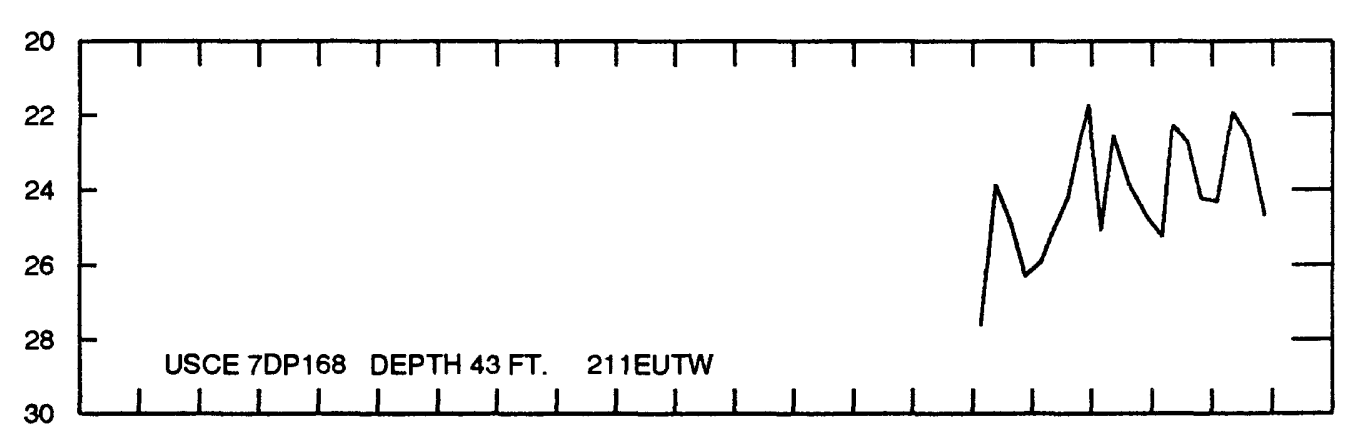

HYDROGRAPHS OF TENNESSEE-TOMBIGBEE OBSERVATION WELLS 


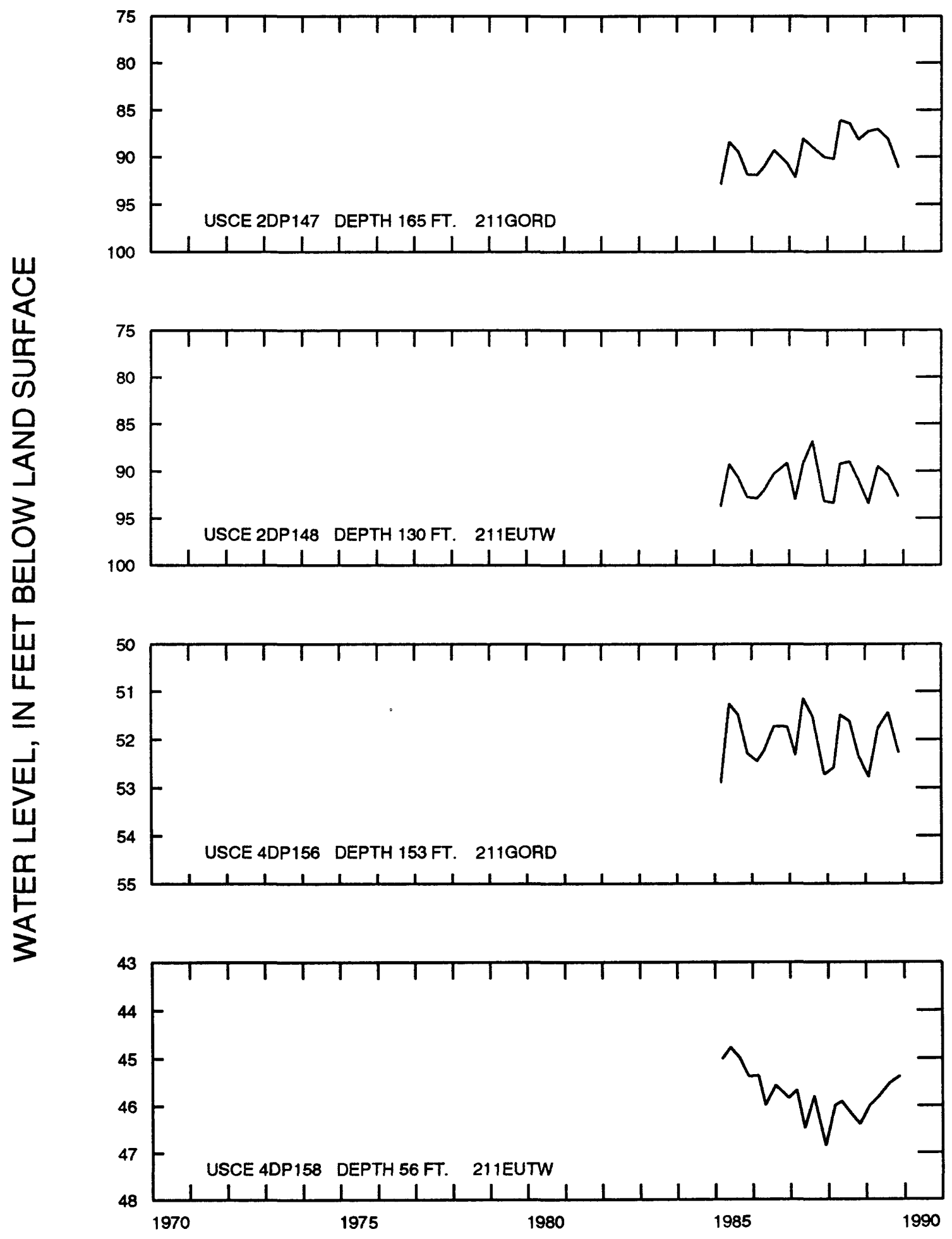

HYDROGRAPHS OF TENNESSEE-TOMBIGBEE OBSERVATION WELLS 


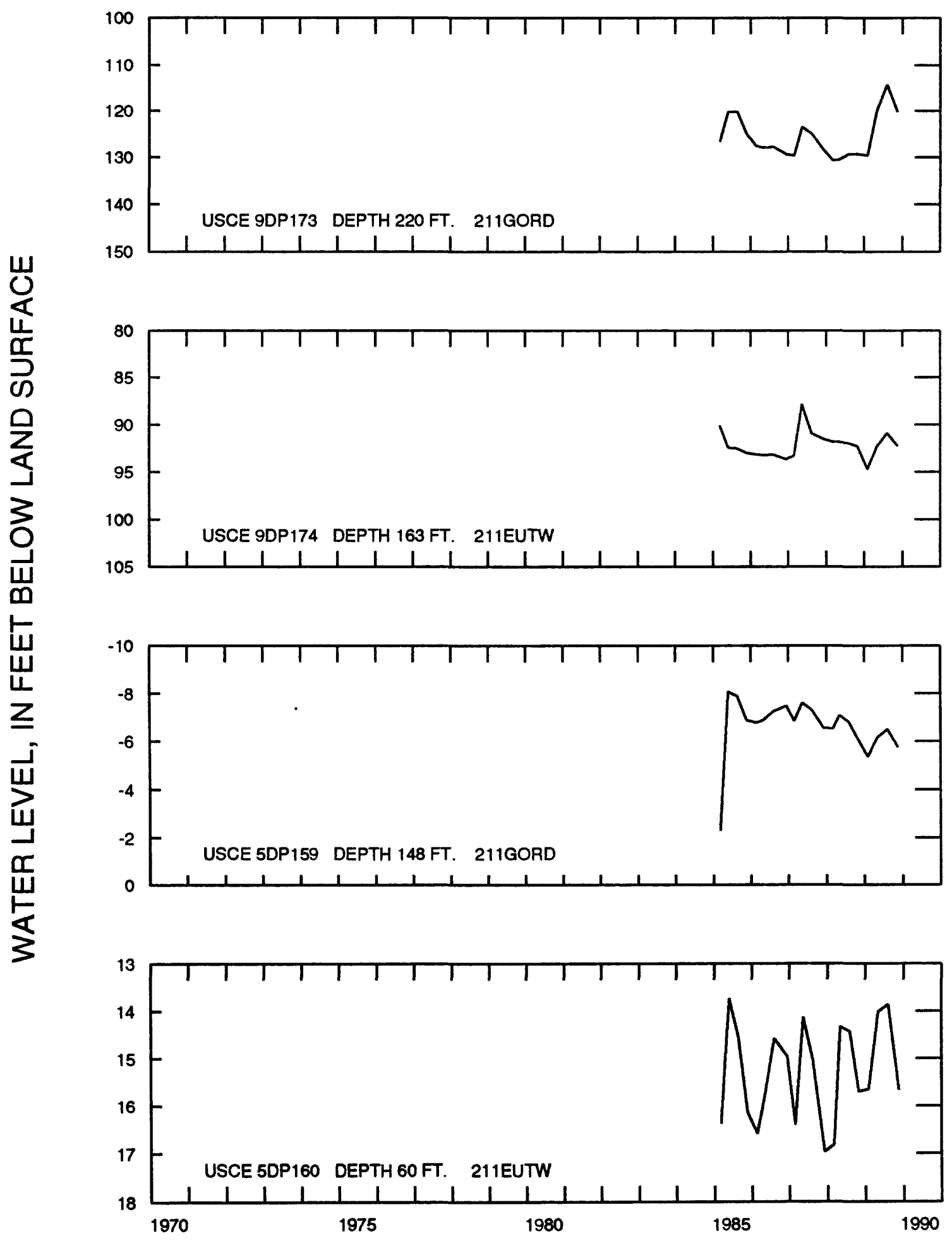

HYDROGRAPHS OF TENNESSEE-TOMBIGBEE OBSERVATION WELLS 


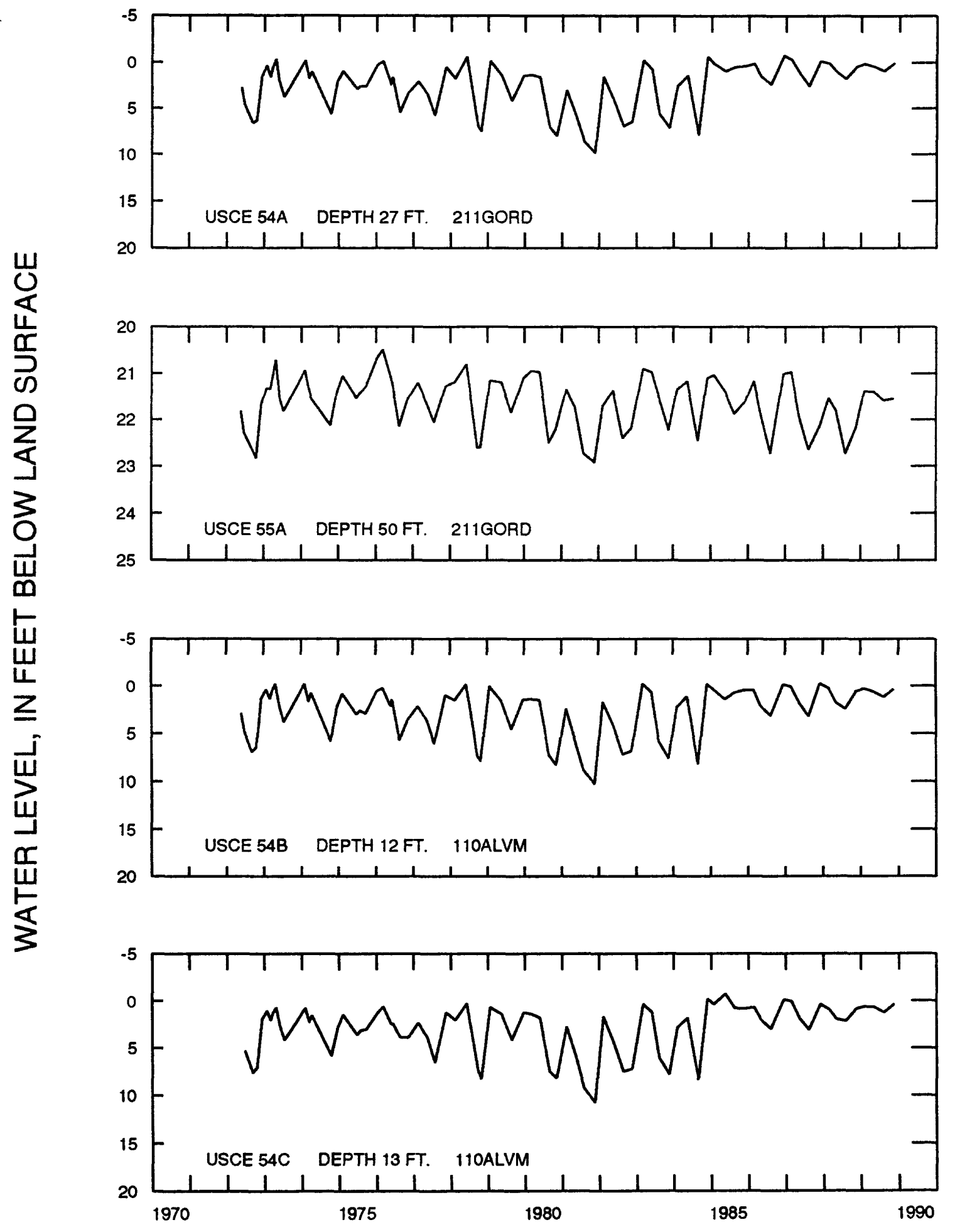

HYDROGRAPHS OF TENNESSEE-TOMBIGBEE OBSERVATION WELLS 


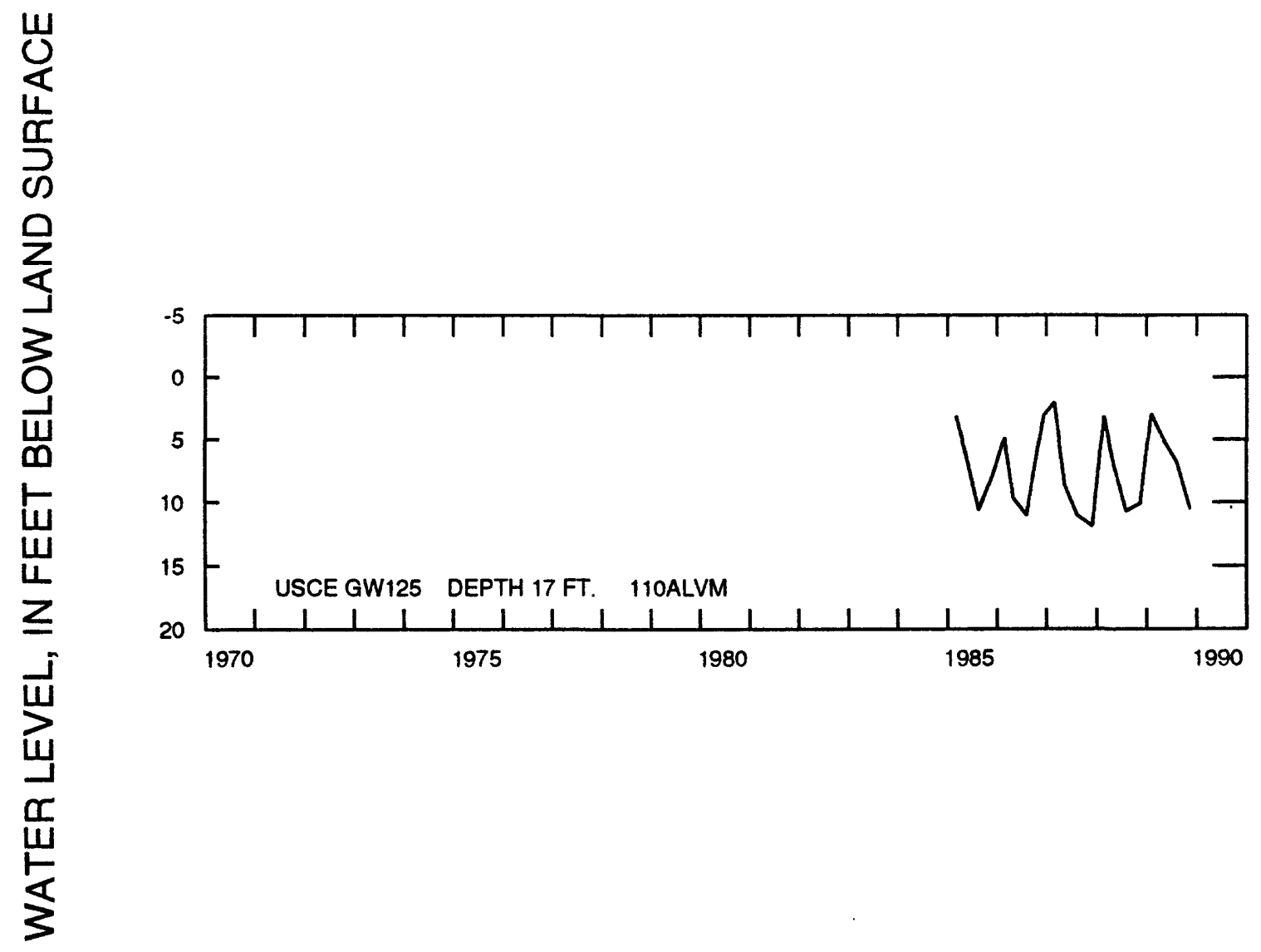

HYDROGRAPHS OF TENNESSEE-TOMBIGBEE OBSERVATION WELLS 


\title{
APPENDIX A
}

\section{GROUND-WATER DATA}

\author{
WATER-QUALITY ANALYSES
}

69

PAGE 71 FOLLOWS 
GROUND-WATER WELLS

343201088204703 M017 USCE $43 C$

\begin{tabular}{|c|c|c|c|c|c|c|c|c|c|c|}
\hline DATE & TIME & $\begin{array}{l}\text { GEO- } \\
\text { LOGIC } \\
\text { UNIT }\end{array}$ & $\begin{array}{l}\text { SAMPLE } \\
\text { SOURCE }\end{array}$ & $\begin{array}{l}\text { SAM- } \\
\text { PLING } \\
\text { CONDI- } \\
\text { TION }\end{array}$ & $\begin{array}{l}\text { DEPTH } \\
\text { BELOW } \\
\text { LAND } \\
\text { SURFACE } \\
\text { (WATER } \\
\text { LEVEL) } \\
\text { (FEET) }\end{array}$ & $\begin{array}{l}\text { DEPTH } \\
\text { OF } \\
\text { WELL, } \\
\text { TOTAL } \\
\text { (FEET) }\end{array}$ & $\begin{array}{c}\text { ELEV. } \\
\text { OF IAAND } \\
\text { SURFACE } \\
\text { DATUM } \\
\text { (FT. } \\
\text { ABOVE } \\
\text { NGVD) }\end{array}$ & $\begin{array}{c}\text { PUMP } \\
\text { OR FIOW } \\
\text { PERIOD } \\
\text { PRIOR } \\
\text { TO SAM- } \\
\text { PLING } \\
\text { (MIN) }\end{array}$ & $\begin{array}{l}\text { F } \\
\text { FLOW } \\
\text { RATE, } \\
\text { INSTAN- } \\
\text { TANEOUS } \\
(G / M)\end{array}$ & $\begin{array}{l}\text { SPE- } \\
\text { CIFIC } \\
\text { CON- } \\
\text { DUCT- } \\
\text { ANCE } \\
\text { (US/CM) }\end{array}$ \\
\hline \multicolumn{11}{|l|}{ APR 1990} \\
\hline DATE & $\begin{array}{c}\text { PH } \\
\text { (STAND- } \\
\text { ARD } \\
\text { UNITS) }\end{array}$ & $\begin{array}{c}\text { TEMPER- } \\
\text { ATURE } \\
\text { WATER } \\
\text { (DEG C) }\end{array}$ & $\begin{array}{l}\text { COLOR } \\
\text { (PLAT- } \\
\text { INUM- } \\
\text { COBALT } \\
\text { UNITS) }\end{array}$ & $\begin{array}{l}\text { HARD- } \\
\text { NESS } \\
\text { TOTAL } \\
\text { (MG/L } \\
\text { AS } \\
\text { CACO3) }\end{array}$ & $\begin{array}{l}\text { CALCIUM } \\
\text { DIS- } \\
\text { SOLVED } \\
\text { (MG/L } \\
\text { AS CA) }\end{array}$ & $\begin{array}{l}\text { MAGNE- } \\
\text { SIUM, } \\
\text { DIS- } \\
\text { SOLVED } \\
\text { (MG/L } \\
\text { AS MG) }\end{array}$ & $\begin{array}{l}\text { SODIUM, } \\
\text { DIS- } \\
\text { SOLVED } \\
\text { (MG/L } \\
\text { AS NA) }\end{array}$ & $\begin{array}{l}\text { SODIUM } \\
\text { PERCENT }\end{array}$ & $\begin{array}{l}\text { SODIUM } \\
\text { AD- } \\
\text { SORP- } \\
\text { TION } \\
\text { RATIO }\end{array}$ & $\begin{array}{l}\text { POTAS- } \\
\text { SIUM, } \\
\text { DIS- } \\
\text { SOLVED } \\
\text { (MG/L } \\
\text { AS K) }\end{array}$ \\
\hline \multirow[t]{2}{*}{$\begin{array}{l}\text { APR } \\
\quad 11 \ldots\end{array}$} & 5.7 & 16.5 & 10 & 4 & 1.3 & 0.30 & 1.0 & 29 & 0.2 & 0.60 \\
\hline & $\begin{array}{c}\text { ALKA- } \\
\text { LINITY } \\
\text { LAB } \\
\text { (MG/I } \\
\text { AS } \\
\text { CACO3) }\end{array}$ & $\begin{array}{l}\text { SULFATE } \\
\text { DIS- } \\
\text { SOLVED } \\
\text { (MG/I } \\
\text { AS SO4) }\end{array}$ & $\begin{array}{l}\text { CHLO- } \\
\text { RIDE, } \\
\text { DIS- } \\
\text { SOLVED } \\
\text { (MG/I } \\
\text { AS CL) }\end{array}$ & $\begin{array}{l}\text { FLUO- } \\
\text { RIDE, } \\
\text { DIS- } \\
\text { SOLVED } \\
\text { (MG/I } \\
\text { AS E) }\end{array}$ & $\begin{array}{l}\text { SILICA, } \\
\text { DIS- } \\
\text { SOLVED } \\
\text { (MG/L } \\
\text { AS } \\
\text { SIO2) }\end{array}$ & $\begin{array}{l}\text { SOLIDS, } \\
\text { RESIDUE } \\
\text { AT } 180 \\
\text { DEG. C } \\
\text { DIS- } \\
\text { SOLVED } \\
\text { (MG/L) }\end{array}$ & $\begin{array}{l}\text { SOLIDS, } \\
\text { SUM OF } \\
\text { CONSTI- } \\
\text { TUENTS, } \\
\text { DIS- } \\
\text { SOLVED } \\
\text { (MG /L) }\end{array}$ & $\begin{array}{c}\text { NITRO- } \\
\text { GEN, } \\
\text { NO2+NO3 } \\
\text { DIS- } \\
\text { SOLVED } \\
\text { (MG/I } \\
\text { AS N) }\end{array}$ & $\begin{array}{l}\text { IRON, } \\
\text { DIS- } \\
\text { SOLVED } \\
\text { (UG/L } \\
\text { AS FE) }\end{array}$ & $\begin{array}{l}\text { MANGA- } \\
\text { NESE, } \\
\text { DIS- } \\
\text { SOLVED } \\
\text { (UG/L } \\
\text { AS MN) }\end{array}$ \\
\hline \multicolumn{11}{|l|}{ APR } \\
\hline & & & 3432 & 1088204 & M018 & USCE 43B & & & & \\
\hline DATE & TIME & $\begin{array}{l}\text { GEO- } \\
\text { LOGIC } \\
\text { UNIT }\end{array}$ & $\begin{array}{l}\text { SAMP LE } \\
\text { SOURCE }\end{array}$ & $\begin{array}{l}\text { SAM- } \\
\text { PLING } \\
\text { CONDI- } \\
\text { TION }\end{array}$ & $\begin{array}{l}\text { DEPTH } \\
\text { BELOW } \\
\text { LAND } \\
\text { SURFACE } \\
\text { (WATER } \\
\text { LEVEL) } \\
\text { (FEET) }\end{array}$ & $\begin{array}{l}\text { DEPTH } \\
\text { OF } \\
\text { WELL, } \\
\text { TOTAL } \\
\text { (FEET) }\end{array}$ & $\begin{array}{c}\text { ELEV. } \\
\text { OF LAND } \\
\text { SURFACE } \\
\text { DATUM } \\
\text { (FT. } \\
\text { ABOVE } \\
\text { NGVD) }\end{array}$ & $\begin{array}{c}\text { PUMP } \\
\text { OR FLOW } \\
\text { PERIOD } \\
\text { PRIOR } \\
\text { TO SAM- } \\
\text { PIING } \\
\text { (MIN) }\end{array}$ & $\begin{array}{l}\text { SPE- } \\
\text { CIFIC } \\
\text { CON- } \\
\text { DUCT- } \\
\text { ANCE } \\
\text { (US/CM) }\end{array}$ & $\begin{array}{c}\text { PH } \\
\text { (STAND- } \\
\text { ARD } \\
\text { UNITS) }\end{array}$ \\
\hline $\begin{array}{c}\text { APR } 1990 \\
11 \ldots\end{array}$ & 1106 & $211 \mathrm{MCSN}$ & 1 & 8.00 & 41.20 & 120.00 & 460 & 26 & 42 & 5.7 \\
\hline DATE & $\begin{array}{l}\text { TEMPER- } \\
\text { ATURE } \\
\text { WATER } \\
\text { (DEG C) }\end{array}$ & $\begin{array}{l}\text { COLOR } \\
\text { (PLAT- } \\
\text { INUM- } \\
\text { COBALT } \\
\text { UNITS) }\end{array}$ & $\begin{array}{l}\text { HARD- } \\
\text { NESS } \\
\text { TOTAL } \\
\text { (MG/L } \\
\text { AS } \\
\text { CACO3) }\end{array}$ & $\begin{array}{l}\text { CALCIUM } \\
\text { DIS- } \\
\text { SOLVED } \\
\text { (MG/I } \\
\text { AS CA) }\end{array}$ & $\begin{array}{l}\text { MAGNE- } \\
\text { SIUM, } \\
\text { DIS- } \\
\text { SOLVED } \\
\text { (MG/L } \\
\text { AS MG) }\end{array}$ & $\begin{array}{l}\text { SODIUM, } \\
\text { DIS- } \\
\text { SOIVED } \\
\text { (MG/L } \\
\text { AS NA) }\end{array}$ & $\begin{array}{l}\text { SODIUM } \\
\text { PERCENT }\end{array}$ & $\begin{array}{l}\text { SODIUM } \\
\text { AD- } \\
\text { SORP- } \\
\text { TION } \\
\text { RATIO }\end{array}$ & $\begin{array}{l}\text { POTAS- } \\
\text { SIUM, } \\
\text { DIS- } \\
\text { SOLVED } \\
\text { (MG/I } \\
\text { AS K) }\end{array}$ & $\begin{array}{c}\text { ALKA- } \\
\text { LINITY } \\
\text { LAB } \\
\text { (MG/L } \\
\text { AS } \\
\text { CACO3) }\end{array}$ \\
\hline
\end{tabular}

\begin{tabular}{|c|c|c|c|c|c|c|c|c|c|}
\hline $\begin{array}{l}\text { APR } \\
\quad 11 \ldots\end{array}$ & 16.5 & 10 & 15 & 4.2 & 1.0 & 1.0 & 12 & 0.1 & 1.0 \\
\hline DATE & $\begin{array}{l}\text { SULFATE } \\
\text { DIS- } \\
\text { SOLVED } \\
\text { (MG/L } \\
\text { AS SO4) }\end{array}$ & $\begin{array}{l}\text { CHLO- } \\
\text { RIDE, } \\
\text { DIS- } \\
\text { SOIVED } \\
\text { (MG/L } \\
\text { AS CI) }\end{array}$ & $\begin{array}{l}\text { FLUO- } \\
\text { RIDE, } \\
\text { DIS- } \\
\text { SOLVED } \\
\text { (MG/L } \\
\text { AS F) }\end{array}$ & $\begin{array}{c}\text { SIIICA, } \\
\text { DIS- } \\
\text { SOIVED } \\
\text { (MG/I } \\
\text { AS } \\
\text { SIO2) }\end{array}$ & $\begin{array}{l}\text { SOLIDS, } \\
\text { RESIDUE } \\
\text { AT } 180 \\
\text { DEG. C } \\
\text { DIS- } \\
\text { SOLVED } \\
\text { (MG/L) }\end{array}$ & $\begin{array}{c}\text { SOLIDS, } \\
\text { SUM OF } \\
\text { CONSTI- } \\
\text { TUENTS, } \\
\text { DIS- } \\
\text { SOLVED } \\
\text { (MG/L) }\end{array}$ & $\begin{array}{c}\text { NITRO- } \\
\text { GEN, } \\
\text { NO2+NO3 } \\
\text { DIS- } \\
\text { SOIVED } \\
\text { (MG/L } \\
\text { AS N) }\end{array}$ & $\begin{array}{l}\text { IRON, } \\
\text { DIS- } \\
\text { SOLVED } \\
\text { (UG/I } \\
\text { AS FE) }\end{array}$ & $\begin{array}{l}\text { MANGA- } \\
\text { NESE, } \\
\text { DIS- } \\
\text { SOLVED } \\
\text { (UG/L } \\
\text { AS MN) }\end{array}$ \\
\hline
\end{tabular}

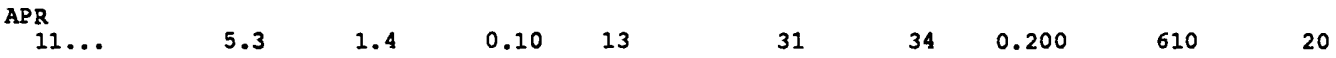




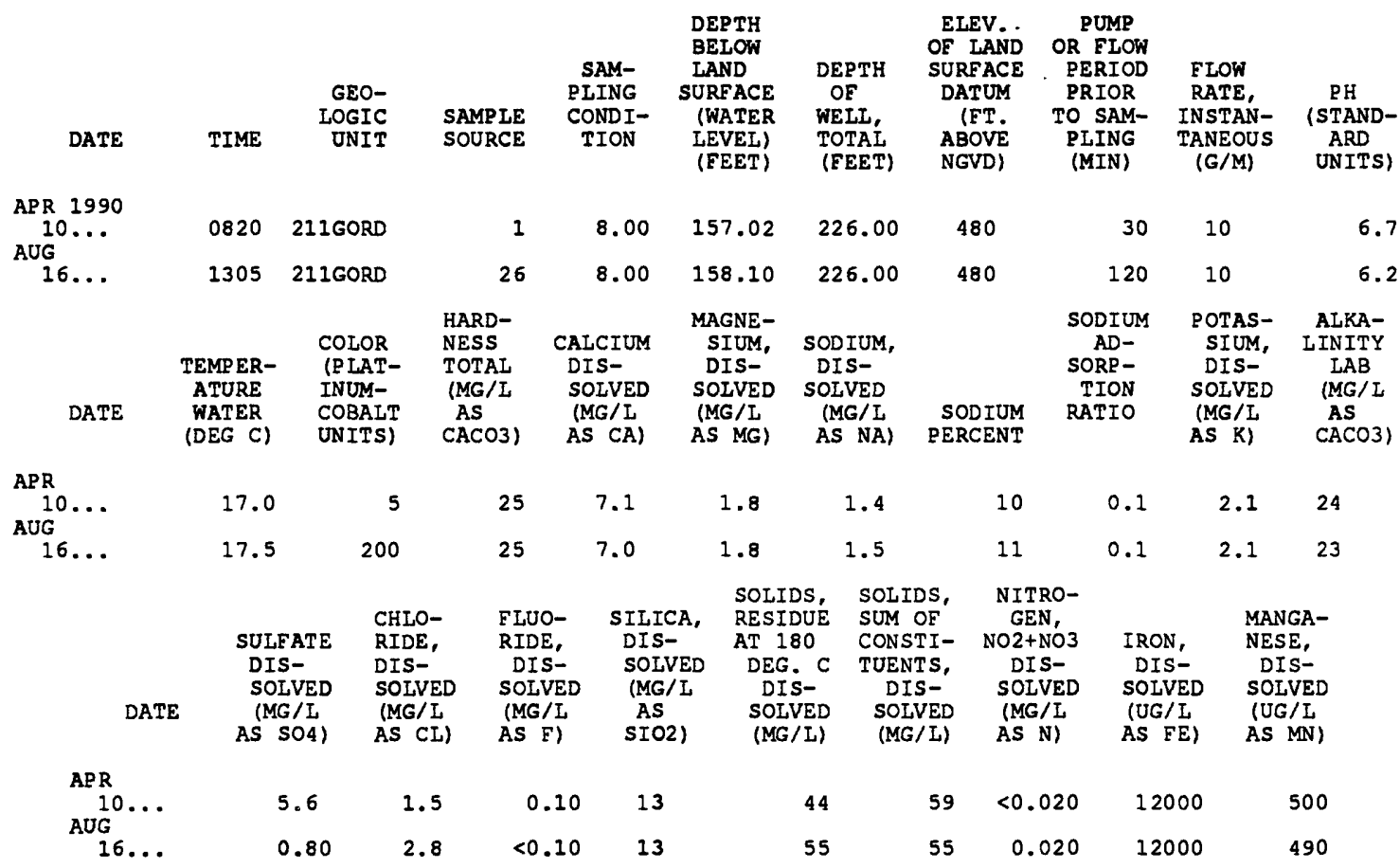

343152088213003 M022 USCE 41B

\begin{tabular}{|c|c|c|c|c|c|c|c|c|c|c|}
\hline DATE & TIME & $\begin{array}{r}\text { GEO- } \\
\text { LOGIC } \\
\text { UNIT }\end{array}$ & $\begin{array}{l}\text { SAMPIE } \\
\text { SOURCE }\end{array}$ & $\begin{array}{l}\text { SAM- } \\
\text { PIING } \\
\text { CONDI- } \\
\text { TION }\end{array}$ & $\begin{array}{l}\text { DEPTH } \\
\text { BELOW } \\
\text { LAND } \\
\text { SURFACE } \\
\text { (WATER } \\
\text { LEVEL) } \\
\text { (FEET) }\end{array}$ & $\begin{array}{l}\text { DEPTH } \\
\text { OF } \\
\text { WELI, } \\
\text { TOTAL } \\
\text { (FEET) }\end{array}$ & $\begin{array}{l}\text { ELEV. } \\
\text { OE LAND } \\
\text { SURFACE } \\
\text { DATUM } \\
\text { (ET. } \\
\text { ABOVE } \\
\text { NGVD) }\end{array}$ & $\begin{array}{c}\text { PUMP } \\
\text { OR ELOW } \\
\text { PERIOD } \\
\text { PRIOR } \\
\text { TO SAM- } \\
\text { PLING } \\
\text { (MIN) }\end{array}$ & $\begin{array}{l}\text { ELOW } \\
\text { RATE, } \\
\text { INSTAN- } \\
\text { TANEOUS } \\
\text { (G/M) }\end{array}$ & $\begin{array}{l}\text { SPE- } \\
\text { CIFIC } \\
\text { CON- } \\
\text { DUCT- } \\
\text { ANCE } \\
\text { (US/CM) }\end{array}$ \\
\hline $\begin{array}{c}\text { APR } 1990 \\
10 \ldots\end{array}$ & 0915 & $211 \mathrm{MCSN}$ & 1 & 8.00 & 72.22 & 176.00 & 480 & 30 & 10 & 42 \\
\hline DATE & $\begin{array}{c}\text { PH } \\
\text { (STAND- } \\
\text { ARD } \\
\text { UNITS) }\end{array}$ & $\begin{array}{c}\text { TEMPER- } \\
\text { ATURE } \\
\text { WATER } \\
\text { (DEG C) }\end{array}$ & $\begin{array}{l}\text { COLOR } \\
\text { (PLAT- } \\
\text { INUM- } \\
\text { COBALT } \\
\text { UNITS) }\end{array}$ & $\begin{array}{l}\text { HARD- } \\
\text { NESS } \\
\text { TOTAL } \\
\text { (MG/L } \\
\text { AS } \\
\text { CACO3) }\end{array}$ & $\begin{array}{l}\text { CALCIUM } \\
\text { DIS- } \\
\text { SOLVED } \\
\text { (MG/L } \\
\text { AS CA) }\end{array}$ & $\begin{array}{l}\text { MAGNE- } \\
\text { SIUM, } \\
\text { DIS- } \\
\text { SOLVED } \\
\text { (MG/I } \\
\text { AS MG) }\end{array}$ & $\begin{array}{l}\text { SODIUM, } \\
\text { DIS- } \\
\text { SOLVED } \\
\text { (MG/L } \\
\text { AS NA) }\end{array}$ & $\begin{array}{r}\text { SODIUM } \\
\text { PERCENT }\end{array}$ & $\begin{array}{c}\text { SODIUM } \\
\text { AD- } \\
\text { SORP- } \\
\text { TION } \\
\text { RATIO }\end{array}$ & $\begin{array}{l}\text { POTAS- } \\
\text { SIUM, } \\
\text { DIS- } \\
\text { SOLVED } \\
\text { (MG/L } \\
\text { AS K) }\end{array}$ \\
\hline $\begin{array}{l}\text { APR } \\
10 \ldots\end{array}$ & 6.2 & 17.0 & $<5$ & 16 & 4.7 & 1.0 & 1.1 & 12 & 0.1 & 1.6 \\
\hline DATE & $\begin{array}{c}\text { ALKA- } \\
\text { LINITY } \\
\text { IAB } \\
\text { (MG/L } \\
\text { AS } \\
\text { CACO3) }\end{array}$ & $\begin{array}{l}\text { SULEATE } \\
\text { DIS- } \\
\text { SOLVED } \\
\text { (MG/L } \\
\text { AS SO4) }\end{array}$ & $\begin{array}{l}\text { CHLO- } \\
\text { RIDE, } \\
\text { DIS- } \\
\text { SOLVED } \\
\text { (MG/I } \\
\text { AS CL) }\end{array}$ & $\begin{array}{l}\text { ELUO- } \\
\text { RIDE, } \\
\text { DIS- } \\
\text { SOLVED } \\
\text { (MG/L } \\
\text { AS F) }\end{array}$ & $\begin{array}{l}\text { SILICA, } \\
\text { DIS- } \\
\text { SOLVED } \\
\text { (MG/L } \\
\text { AS } \\
\text { SIO2) }\end{array}$ & $\begin{array}{c}\text { SOLIDS, } \\
\text { RESIDUE } \\
\text { AT } 180 \\
\text { DEG. C } \\
\text { DIS- } \\
\text { SOLVED } \\
\text { (MG/L) }\end{array}$ & $\begin{array}{l}\text { SOLIDS, } \\
\text { SUM OF } \\
\text { CONSTI- } \\
\text { TUENTS, } \\
\text { DIS- } \\
\text { SOLVED } \\
\text { (MG/L) }\end{array}$ & $\begin{array}{c}\text { NITRO- } \\
\text { GEN, } \\
\text { NO2+NO3 } \\
\text { DIS- } \\
\text { SOLVED } \\
\text { (MG/L } \\
\text { AS N) }\end{array}$ & $\begin{array}{l}\text { IRON, } \\
\text { DIS- } \\
\text { SOLVED } \\
\text { (UG/L } \\
\text { AS FE) }\end{array}$ & $\begin{array}{l}\text { MANGA- } \\
\text { NESE, } \\
\text { DIS- } \\
\text { SOIVED } \\
\text { (UG/L } \\
\text { AS MN) }\end{array}$ \\
\hline
\end{tabular}
APR

10.

15

$\begin{array}{llll}3.9 & 1.0 & 0.10 & 21\end{array}$

38

$44<0.020$

390

20 
GROUND-WATER WELLS--Cont Inued

343201088194701 M025 USCE 43D

\begin{tabular}{|c|c|c|c|c|c|c|c|c|c|c|}
\hline DATE & TIME & $\begin{array}{l}\text { GEO- } \\
\text { LOGIC } \\
\text { UNIT }\end{array}$ & $\begin{array}{l}\text { SAMPLE } \\
\text { SOURCE }\end{array}$ & $\begin{array}{l}\text { SAM- } \\
\text { PLING } \\
\text { CONDI- } \\
\text { TION }\end{array}$ & $\begin{array}{c}\text { DEPTH } \\
\text { BELOW } \\
\text { IAND } \\
\text { SURFACE } \\
\text { (WATER } \\
\text { IEVEL) } \\
\text { (EEET) }\end{array}$ & $\begin{array}{l}\text { DEPTH } \\
\text { OF } \\
\text { WELL, } \\
\text { TOTAL } \\
\text { (EEET) }\end{array}$ & $\begin{array}{c}\text { ELEV. } \\
\text { OF LAND } \\
\text { SURFACE } \\
\text { DATUM } \\
\text { (FT. } \\
\text { ABOVE } \\
\text { NGVD) }\end{array}$ & $\begin{array}{c}\text { PUMP } \\
\text { OR FLOW } \\
\text { PERIOD } \\
\text { PRIOR } \\
\text { TO SAM- } \\
\text { PLING } \\
\text { (MIN) }\end{array}$ & $\begin{array}{c}\text { FLOW } \\
\text { RATE, } \\
\text { INSTAN- } \\
\text { TANEOUS } \\
(G / M)\end{array}$ & $\begin{array}{l}\text { SPE- } \\
\text { CIFIC } \\
\text { CON- } \\
\text { DUCT- } \\
\text { ANCE } \\
\text { (US/CM) }\end{array}$ \\
\hline $\begin{array}{c}\text { APR } 1990 \\
11 \ldots\end{array}$ & 1230 & 211EUTWR & 1 & 8.00 & 43.28 & 118.00 & 460 & 30 & 5.0 & 20 \\
\hline DATE & $\begin{array}{c}\text { PH } \\
\text { (STAND- } \\
\text { ARD } \\
\text { UNITS) }\end{array}$ & $\begin{array}{c}\text { TEMPER- } \\
\text { ATURE } \\
\text { WATER } \\
\text { (DEG C) }\end{array}$ & $\begin{array}{l}\text { COLOR } \\
\text { (PLAT- } \\
\text { INUM- } \\
\text { COBALT } \\
\text { UNITS) }\end{array}$ & $\begin{array}{l}\text { HARD- } \\
\text { NESS } \\
\text { TOTAL } \\
\text { (MG/L } \\
\text { AS } \\
\text { CACO3) }\end{array}$ & $\begin{array}{l}\text { CALCIUM } \\
\text { DIS- } \\
\text { SOLVED } \\
\text { (MG/L } \\
\text { AS CA) }\end{array}$ & $\begin{array}{c}\text { MAGNE- } \\
\text { SIUM, } \\
\text { DIS- } \\
\text { SOLVED } \\
\text { (MG/L } \\
\text { AS MG) }\end{array}$ & $\begin{array}{l}\text { SODIUM, } \\
\text { DIS- } \\
\text { SOLVED } \\
\text { (MG/L } \\
\text { AS NA) }\end{array}$ & $\begin{array}{l}\text { SODIUM } \\
\text { PERCENT }\end{array}$ & $\begin{array}{c}\text { SODIUM } \\
\text { AD- } \\
\text { SORP- } \\
\text { TION } \\
\text { RATIO }\end{array}$ & $\begin{array}{l}\text { POTAS- } \\
\text { SIUM, } \\
\text { DIS- } \\
\text { SOLVED } \\
\text { (MG/L } \\
\text { AS K) }\end{array}$ \\
\hline $\begin{array}{l}\text { PR } \\
11 \ldots\end{array}$ & 6.0 & 18.0 & $<5$ & 7 & 2.0 & 0.40 & 0.90 & 21 & 0.2 & 0.50 \\
\hline DATE & $\begin{array}{c}\text { ALKA- } \\
\text { LINITY } \\
\text { IAB } \\
\text { (MG/L } \\
\text { AS } \\
\text { CACO3) }\end{array}$ & $\begin{array}{l}\text { SULFATE } \\
\text { DIS- } \\
\text { SOLVED } \\
\text { (MG/L } \\
\text { AS SO4) }\end{array}$ & $\begin{array}{l}\text { CHLO- } \\
\text { RIDE, } \\
\text { DIS- } \\
\text { SOLVED } \\
\text { (MG/L } \\
\text { AS CL) }\end{array}$ & $\begin{array}{l}\text { FLUO- } \\
\text { RIDE, } \\
\text { DIS- } \\
\text { SOLVED } \\
\text { (MG/L } \\
\text { AS F) }\end{array}$ & $\begin{array}{l}\text { SILICA, } \\
\text { DIS- } \\
\text { SOLVED } \\
\text { (MG/L } \\
\text { AS } \\
\text { SIO2) }\end{array}$ & $\begin{array}{l}\text { SOLIDS, } \\
\text { RESIDUE } \\
\text { AT } 180 \\
\text { DEG. C } \\
\text { DIS- } \\
\text { SOLVED } \\
\text { (MG/L) }\end{array}$ & $\begin{array}{c}\text { SOLIDS, } \\
\text { SUM OF } \\
\text { CONSTI- } \\
\text { TUENTS, } \\
\text { DIS- } \\
\text { SOLVED } \\
(M G / L)\end{array}$ & $\begin{array}{c}\text { NITRO- } \\
\text { GEN, } \\
\text { NO2+NO3 } \\
\text { DIS- } \\
\text { SOLVED } \\
\text { (MG/L } \\
\text { AS N) }\end{array}$ & $\begin{array}{l}\text { IRON, } \\
\text { DIS- } \\
\text { SOLVED } \\
\text { (UG/I } \\
\text { AS FE) }\end{array}$ & $\begin{array}{l}\text { MANGA- } \\
\text { NESE, } \\
\text { DIS- } \\
\text { SOLVED } \\
\text { (UG/L } \\
\text { AS MN) }\end{array}$ \\
\hline
\end{tabular}

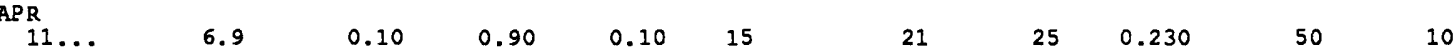




\section{APPENDIX B}

\section{SURFACE-WATER DATA}

PAGE 77 FOLLOWS 


\section{APPENDIX B}

\section{SURFACE-WATER DATA}

DESCRIPTIONS OF SITES

PAGE 79 FOLLOWS 
SURFACE-WATER NETWORK

03592824 02433000 02436500 333927088304935

02439600

02441000

02441400

02441498

02443500

02443610

02444210

02446500

02447010

02448500

02467001

02430500

02430680

02430690

02431000

02431410

02433500

02437000

02437500

02439400

332929088273300

332751088261000

02.444157

02444500

02448000

02449000

02469525

02469762
TENNESSEE-TOMBIGBEE WATERWAY AT CROSS ROADS, MS BULL MOUNTAIN CREEK NEAR SMITHVILLE, MS

TOWN CREEK NEAR NETTLETON, MS

TTW COLUMBUS LAKE BUTTAHATCHEE RIVER BEND SR $26 \mathrm{~A}$

BUTTAHATCHEE RIVER NEAR KOLOLA SPRINGS, MS

\section{1}

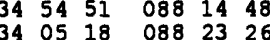

$\begin{array}{llllll}34 & 03 & 32 & 088 & 37 & 40\end{array}$

$\begin{array}{llllll}33 & 39 & 27 & 088 & 30 & 49\end{array}$

33 40 24

$\begin{array}{lll}33 & 32 & 17\end{array}$

$\begin{array}{lll}33 & 32 & 17 \\ 33 & 29 & 40\end{array}$

TIBBEE CREEK NEAR TIBBEE, MS

TOMBIGBEE RIVER NEAR COLUMBUS, MS

$\begin{array}{llll}088 & 38 & 00\end{array}$

COLUBS, MS $\begin{array}{llllll}33 & 29 & 40 & 088 & 27 & 40\end{array}$

LUXAPALLI RIVER COLUMBUS BEND SR 11B AT COLUMBUS, MS 332606

TOMBIGBEE RIVER PRATT CAMP SR 5HB BELOW COLUMBUS, MS 332030

TOMBIGBEE RIVER BIG CREEK BEND NEAR PICKENSVILLE, AL 331111

SIPSEY RIVER NEAR ELROD, AI

$\begin{array}{lll}\text { TOMBIGBEE RIVER COOKS BEND NEAR WARSAW, AL } & 325738 \\ \text { NOXUBEE RIVER NEAR GEIGER, AL } & 325506\end{array}$

TOMBIGBEE RIVER BELOW DEMOPOLIS LOCK AND DAM, AL

325506 SITES NOT IN THE SUREACE-WATER NETWORK

TOMBIGBEE RIVER NEAR MARIETTA, MS

TWENTYMILE CREEK NEAR GUNTOWN, MS

TWENTYMILE CREEK NEAR MANTACHIE, MS

TOMBIGBEE RIVER NEAR FULTON, MS

MANTACHIE CREEK BELOW DORSEY, MS

TOMBIGBEE RIVER AT BIGBEE, MS

TOMBIGBEE RIVER NEAR AMORY, MS

TOMBIGBEE RIVER AT ABERDEEN, MS

BUTTAHATCHEE RIVER NEAR ABERDEEN, MS

TTW ALIVEVILLE LAKE ABOVE COLUMBUS BEND

\section{$\begin{array}{lll}34 & 25 & 35\end{array}$}

$\begin{array}{llllll}34 & 23 & 10 & 088 & 27 & 40\end{array}$

$\begin{array}{llllll}34 & 15 & 53 & 088 & 26 & 42\end{array}$

$\begin{array}{llllll}34 & 13 & 41 & 088 & 27 & 08\end{array}$

$\begin{array}{llllll}34 & 00 & 40 & 088 & 30 & 50\end{array}$

$\begin{array}{llllll}33 & 59 & 07 & 088 & 33 & 03\end{array}$

$\begin{array}{llllll}33 & 49 & 14 & 088 & 31 & 07 \\ 33 & 47 & 24 & 088 & 18 & 55\end{array}$

$\begin{array}{llllll}33 & 29 & 29 & 088 & 27 & 33\end{array}$

TTW ALICEVIIIE LAKE COLUMBUS CUT

$\begin{array}{lllllll}\text { TTW ALICEVILIE LAKE COLUMBUS CUT } & 3327 & 51 & 088 & 26 & 10 \\ \text { TOMBIGBEE RIVER AT ST HWY } 86 \text { NEAR PICKENSVILLE, AL } & 33 & 13 & 35 & 088 & 17 & 29\end{array}$

TOMBIGBEE RIVER NEAR COCHRANE, AL

NOXUBEE RIVER AT MACON, MS

TOMBIGBEE RIVER AT GAINESVILLE, AL

331304

330608

324930

TOMBIGBEE RIVER NEAR NANAFALIA, AL

$\begin{array}{lll}32 & 07 & 48 \\ 31 & 45 & 30\end{array}$
0881416

0883340

$\begin{array}{lll}088 & 09 & 24\end{array}$

$08802 \quad 28$

0880735
06030005

$03160101 \quad 336$

$03160102 \quad 620$

03160101

$03160103 \quad 855$

$03160104 \quad 926$

$03160101 \quad 4450$

03160101

$03160105 \quad 715$

03160106

03160106

$03160107 \quad 528$

03160106

$03160108 \quad 1100$

0316020115400

03160101308

$03160101 \quad 131$

$03160101 \quad 150$

$\begin{array}{lr}03160101 & 612 \\ 03160101 & 66.9\end{array}$

$03160101 \quad 1230$

$03160101 \quad 1930$

031601012170

$03160103 \quad 798$

03160101

03160101

03160106

$03160106 \quad 5940$

$03160108 \quad 768$

$\begin{array}{rr}03160108 & 768 \\ 03160106 & 8630\end{array}$

TOMBIGBEE RIVER BELOW COFEEEVILLE LOCK AND DAM, AL
00

0316020117500

0316020318400 


\section{APPENDIX B}

\section{SURFACE-WATER DATA}

WATER-QUALITY ANALYSES

PAGE 83 FOLLOWS 
03592824 TENNESSEE-TOMBIGBEE NATERWAY AT CROSS ROADS, MS

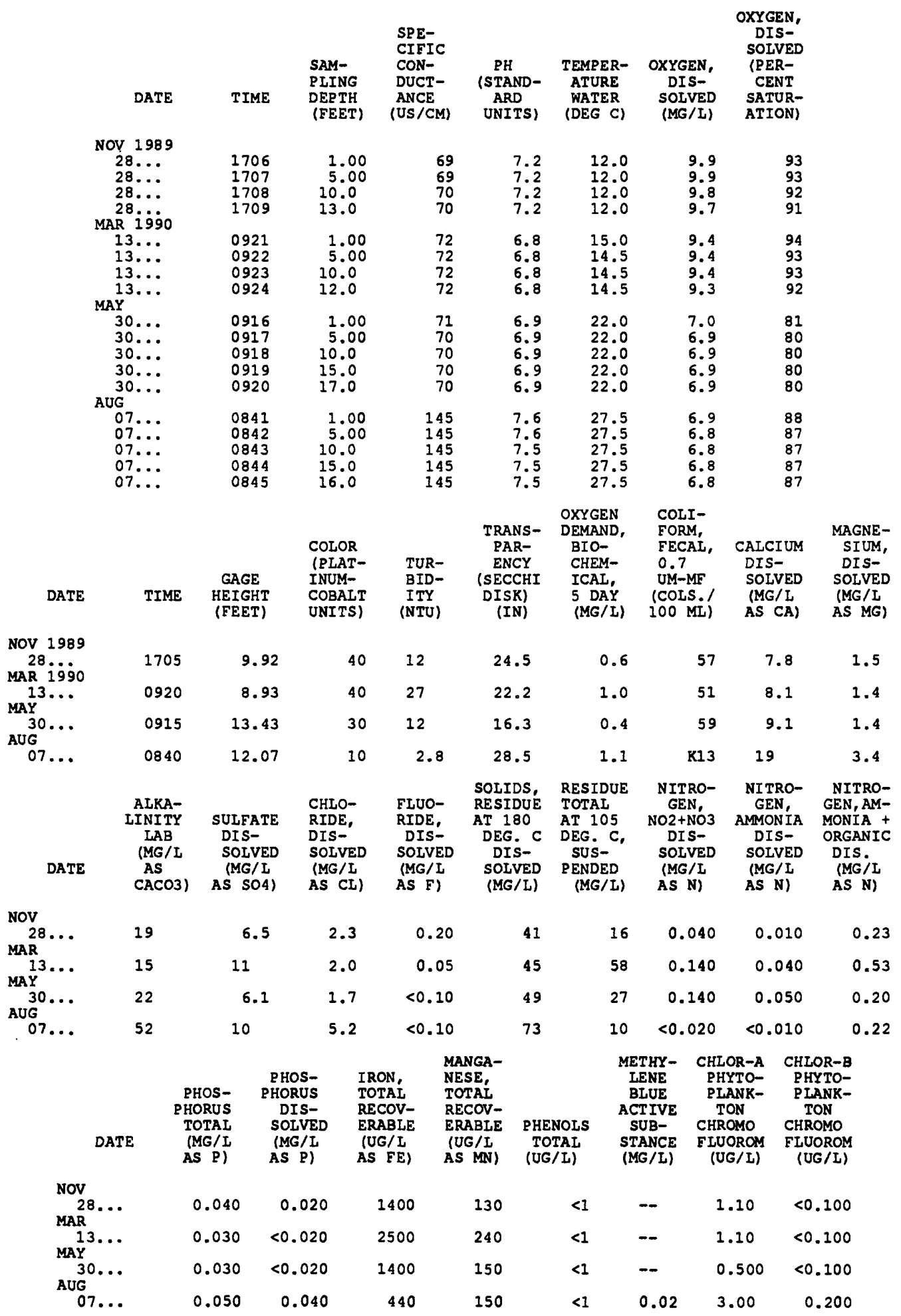


SURFACE-WATER SITES--Cont 1 nued

02430500 TOMBIGBEE RIVER NEAR MARIETTA, MS

\begin{tabular}{|c|c|c|c|c|c|c|c|c|c|}
\hline DATE & TIME & $\begin{array}{c}\text { DIS- } \\
\text { CHARGE, } \\
\text { INST. } \\
\text { CUBIC } \\
\text { FEET } \\
\text { PER } \\
\text { SECOND }\end{array}$ & $\begin{array}{l}\text { SEDI- } \\
\text { MENT, } \\
\text { SUS- } \\
\text { PENDED } \\
\text { (MG/L) }\end{array}$ & $\begin{array}{c}\text { SEDI- } \\
\text { MENT, } \\
\text { DIS- } \\
\text { CHARGE, } \\
\text { SUS- } \\
\text { PENDED } \\
\text { (T/DAY) }\end{array}$ & DATE & TIME & $\begin{array}{c}\text { DIS- } \\
\text { CHARGE, } \\
\text { INST. } \\
\text { CUBIC } \\
\text { FEET } \\
\text { PER } \\
\text { SECOND }\end{array}$ & $\begin{array}{l}\text { SEDI- } \\
\text { MENT, } \\
\text { SUS- } \\
\text { PENDED } \\
\text { (MG/L) }\end{array}$ & $\begin{array}{r}\text { SED } \\
\text { MEN } \\
\text { DI } \\
\text { CHAR } \\
\text { SU } \\
\text { PEN } \\
\text { (T/D }\end{array}$ \\
\hline $\begin{array}{c}\text { MAR } 1990 \\
\text { O6... } \\
\text { APR }\end{array}$ & 1715 & 470 & 61 & 77 & $\begin{array}{l}\text { JUN } \\
28 \ldots \\
\text { AUG }\end{array}$ & 1300 & 128 & 85 & 2 \\
\hline${ }_{M A Y}^{11 \ldots}$ & 1425 & 296 & 57 & 46 & $14 \ldots$ & 1550 & 156 & 113 & \\
\hline $24 \ldots$ & 1530 & 384 & 78 & 81 & & & & & \\
\hline
\end{tabular}

02430680 TWENTYMIIE CREEK NEAR GUNTOWN, MS

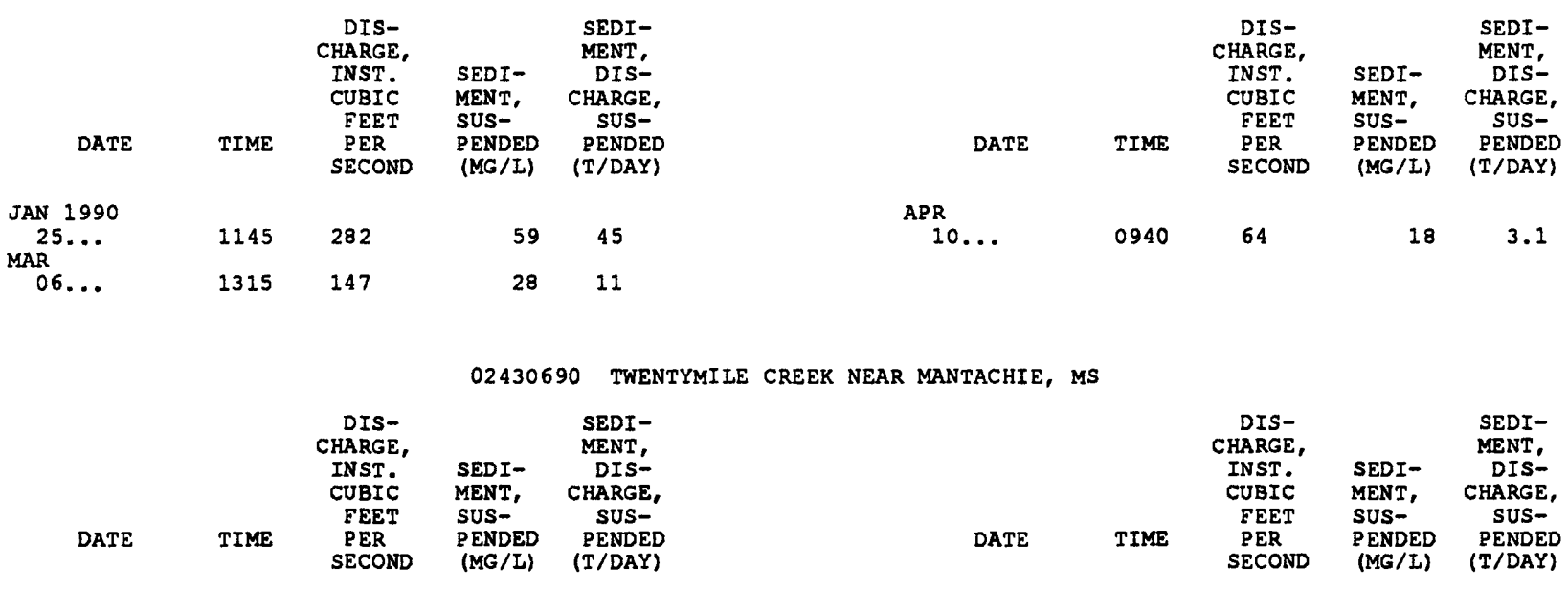

MAY 1990

22 ...

$1525 \quad 415$

$116 \quad 130$

JUL $11 \ldots$

1520

2.4

44

0.29

02431000 TOMBIGBEE RIVER NEAR FULTON, MS

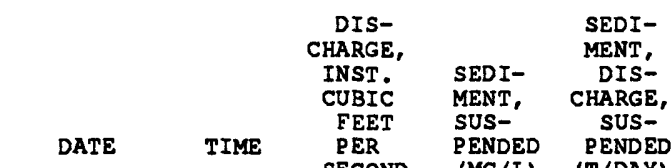

$\begin{array}{crrrr}\begin{array}{crrr}\text { JAN 1990 } \\ 24 \ldots\end{array} & 1645 & 1600 & 147 & 635 \\ \text { FEB } & & & & \\ 02 \ldots & 1530 & 1580 & 822 & 3510 \\ 04 \ldots . . & 0050 & 8960 & 1040 & 25200 \\ 04 \ldots . . & 0635 & 15400 & 1530 & 63600 \\ 04 \ldots . . & 2140 & 24800 & 711 & 47600 \\ 05 \ldots . & 0810 & 17600 & 492 & 23400 \\ 05 \ldots & 1500 & 14100 & 376 & 14300 \\ 16 \ldots & 0220 & 1970 & 154 & 819\end{array}$

\begin{tabular}{|c|c|c|c|c|}
\hline DATE & TIME & $\begin{array}{c}\text { DIS- } \\
\text { CHARGE, } \\
\text { INST. } \\
\text { CUBIC } \\
\text { FEET } \\
\text { PER } \\
\text { SECOND }\end{array}$ & $\begin{array}{l}\text { SEDI- } \\
\text { MENT, } \\
\text { SUS- } \\
\text { PENDED } \\
\text { (MG/L) }\end{array}$ & $\begin{array}{r}\text { SEDI- } \\
\text { MENT, } \\
\text { DIS } \\
\text { CHARGE } \\
\text { SUS } \\
\text { PENDE } \\
\text { (T/DAY }\end{array}$ \\
\hline \multicolumn{5}{|l|}{ MAR } \\
\hline & 1320 & 1560 & 79 & 333 \\
\hline $\operatorname{MAY} 11 \ldots$ & 0725 & 478 & 70 & 90 \\
\hline JUL $21 \ldots$ & 1110 & 1910 & 615 & 3170 \\
\hline AUG & 1600 & 116 & 33 & 10 \\
\hline $14 \ldots$ & 1810 & 157 & 51 & 22 \\
\hline
\end{tabular}


02431410 MANTACHIE CREEK BEIOW DORSEY, MS

\begin{tabular}{|c|c|c|c|c|c|c|c|c|}
\hline DATE & TIME & $\begin{array}{l}\text { DIS- } \\
\text { CHARGE, } \\
\text { INST. } \\
\text { CUBIC } \\
\text { EEET } \\
\text { PER } \\
\text { SECOND }\end{array}$ & $\begin{array}{c}\text { SEDI- } \\
\text { MENT, } \\
\text { DIS- } \\
\text { CHARGE, } \\
\text { SUS- } \\
\text { PENDED } \\
\text { (T/DAY) }\end{array}$ & DATE & TIME & $\begin{array}{l}\text { DIS- } \\
\text { CHARGE, } \\
\text { INST. } \\
\text { CUBIC } \\
\text { EEET } \\
\text { PER } \\
\text { SECOND }\end{array}$ & $\begin{array}{l}\text { SEDI- } \\
\text { MENT, } \\
\text { SUS- } \\
\text { PENDED } \\
\text { (MG/L) }\end{array}$ & $\begin{array}{c}\text { SEDI- } \\
\text { MENT, } \\
\text { DIS- } \\
\text { CHARGE, } \\
\text { SUS- } \\
\text { PENDED } \\
\text { (T/DAY) }\end{array}$ \\
\hline $\begin{array}{l}N 1990 \\
25 \ldots\end{array}$ & 1035 & 231 & 136 & $\begin{array}{l}\text { MAR } \\
07 \ldots \\
\text { APR }\end{array}$ & 1635 & 87 & 37 & 8.7 \\
\hline $\begin{array}{l}02 \ldots \\
02 \ldots \\
03 \ldots \\
04 \ldots \\
04 \ldots \\
04 \ldots \\
05 \ldots \\
15 \ldots \\
16 \ldots \\
16 \ldots \\
16 \ldots \\
16 \ldots\end{array}$ & $\begin{array}{l}1400 \\
1730 \\
2340 \\
0505 \\
0820 \\
1320 \\
0910 \\
2320 \\
0120 \\
0300 \\
0640 \\
0920\end{array}$ & $\begin{array}{r}2100 \\
1290 \\
6170 \\
4680 \\
3500 \\
2170 \\
2210 \\
648 \\
1160 \\
1290 \\
1090 \\
944\end{array}$ & $\begin{array}{rr}1510 & 8560 \\
1050 & 3660 \\
4620 & 77000 \\
2650 & 33500 \\
2160 & 20400 \\
1100 & 6440 \\
172 & 1030 \\
492 & 861 \\
2100 & 6580 \\
1690 & 5890 \\
956 & 2810 \\
662 & 1690\end{array}$ & $\begin{array}{c}09 \ldots \\
\text { MAY } \\
22 \ldots \\
\pi U \\
11 \ldots \\
\text { AUG } \\
13 \ldots\end{array}$ & $\begin{array}{l}1910 \\
1800 \\
1425 \\
1355\end{array}$ & $\begin{array}{r}33 \\
187 \\
9.6 \\
4.3\end{array}$ & $\begin{array}{r}27 \\
110 \\
201 \\
30\end{array}$ & $\begin{array}{c}2.4 \\
56 \\
5.2 \\
0.35\end{array}$ \\
\hline
\end{tabular}

02433000 BULL MOUNTAIN CREEK NEAR SMITHVILLE, MS

\begin{tabular}{|c|c|c|c|c|c|c|c|c|c|c|}
\hline DATE & TIME & $\begin{array}{l}\text { SPE- } \\
\text { CIFIC } \\
\text { CON- } \\
\text { DUCT- } \\
\text { ANCE } \\
\text { (US/CM) }\end{array}$ & $\begin{array}{c}\text { PH } \\
\text { (STAND- } \\
\text { ARD } \\
\text { UNITS) }\end{array}$ & $\begin{array}{c}\text { TEMPER- } \\
\text { ATURE } \\
\text { WATER } \\
\text { (DEG C) }\end{array}$ & $\begin{array}{l}\text { COLOR } \\
\text { (PLAT- } \\
\text { INUM- } \\
\text { COBALT } \\
\text { UNITS) }\end{array}$ & $\begin{array}{l}\text { TUR- } \\
\text { BID- } \\
\text { ITY } \\
\text { (NTU) }\end{array}$ & $\begin{array}{c}\text { TRANS- } \\
\text { PAR- } \\
\text { ENCY } \\
\text { (SECCHI } \\
\text { DISK) } \\
\text { (IN) }\end{array}$ & $\begin{array}{c}\text { OXYGEN, } \\
\text { DIS- } \\
\text { SOLVED } \\
\text { (MG/L) }\end{array}$ & $\begin{array}{l}\text { OXYGEN, } \\
\text { DIS- } \\
\text { SOLVED } \\
\text { (PER- } \\
\text { CENT } \\
\text { SATUR- } \\
\text { ATION) }\end{array}$ & $\begin{array}{l}\text { OXYGEN } \\
\text { DEMAND, } \\
\text { BIO- } \\
\text { CHEM- } \\
\text { ICAL, } \\
5 \text { DAY } \\
\text { (MG/I) }\end{array}$ \\
\hline $\begin{array}{l}\text { NOV } 1989 \\
29.1990 \\
\text { MAR } 1990\end{array}$ & 1305 & 29 & 6.6 & 12.0 & 40 & 9.2 & 28.5 & 9.3 & -- & 0.5 \\
\hline $\operatorname{MAY}^{13} \ldots$ & 1320 & 28 & 6.2 & 17.5 & 30 & 14 & 24.5 & 8.8 & 93 & 0.8 \\
\hline${ }_{A U G}^{30} \cdots$ & 1300 & 26 & 6.1 & 19.5 & 50 & 35 & 11.0 & 7.8 & 86 & 0.8 \\
\hline $07 \ldots$ & 1300 & 39 & 6.6 & 28.0 & 10 & 4.8 & 30.5 & 7.0 & 90 & 0.7 \\
\hline DATE & $\begin{array}{l}\text { COLI- } \\
\text { FORM, } \\
\text { FECAL, } \\
0.7 \\
\text { UM-ME } \\
\text { (COLS.' } \\
100 \mathrm{ML} \text { ) }\end{array}$ & $\begin{array}{l}\text { CALCIUM } \\
\text { DIS- } \\
\text { SOLVED } \\
\text { (MG/L } \\
\text { AS CA) }\end{array}$ & $\begin{array}{l}\text { MAGNE- } \\
\text { SIUM, } \\
\text { DIS- } \\
\text { SOLVED } \\
\text { (MG/L } \\
\text { AS MG) }\end{array}$ & $\begin{array}{c}\text { ALKA- } \\
\text { LINITY } \\
\text { LAB } \\
\text { (MG/I } \\
\text { AS } \\
\text { CACO3) }\end{array}$ & $\begin{array}{l}\text { SULFATE } \\
\text { DIS- } \\
\text { SOLVED } \\
\text { (MG/I } \\
\text { AS SO4) }\end{array}$ & $\begin{array}{l}\text { CHLO- } \\
\text { RIDE, } \\
\text { DIS- } \\
\text { SOLVED } \\
\text { (MG/L } \\
\text { AS CL) }\end{array}$ & $\begin{array}{l}\text { FLUO- } \\
\text { RIDE, } \\
\text { DIS- } \\
\text { SOLVED } \\
\text { (MG/L } \\
\text { AS F) }\end{array}$ & $\begin{array}{l}\text { SOLIDS, } \\
\text { RESIDUE } \\
\text { AT } 180 \\
\text { DEG. C } \\
\text { DIS- } \\
\text { SOLVED } \\
\text { (MG/L) }\end{array}$ & $\begin{array}{l}\text { RESIDUE } \\
\text { TOTAL } \\
\text { AT } 105 \\
\text { DEG. C, } \\
\text { SUS- } \\
\text { PENDED } \\
\text { (MG/L) }\end{array}$ & $\begin{array}{c}\text { NITRO- } \\
\text { GEN, } \\
\text { NO2+NO3 } \\
\text { DIS- } \\
\text { SOLVED } \\
\text { (MG/I } \\
\text { AS N) }\end{array}$ \\
\hline NOV & & & & & & & & & & \\
\hline MAR $29 .$. & 65 & 2.1 & 0.80 & 4.8 & 2.9 & 1.9 & 0.10 & 18 & 11 & 0.130 \\
\hline${ }_{M A Y}^{13} \ldots$ & K82 & 1.7 & 0.80 & -- & 3.1 & 1.6 & $<0.05$ & 27 & 22 & 0.170 \\
\hline AUG $30 \ldots$ & 210 & 1.6 & 0.80 & 4.1 & 2.5 & 1.5 & $<0.10$ & 23 & 45 & 0.180 \\
\hline $07 \ldots$ & 35 & 3.0 & 1.0 & 8.8 & 2.5 & 2.2 & $<0.10$ & 25 & 9 & 0.080 \\
\hline DATE & $\begin{array}{l}\text { NITRO- } \\
\text { GEN, } \\
\text { AMMONIA } \\
\text { DIS- } \\
\text { SOLVED } \\
\text { (MG/L } \\
\text { AS N) }\end{array}$ & $\begin{array}{l}\text { NITRO- } \\
\text { GEN, AM- } \\
\text { MONIA + } \\
\text { ORGANIC } \\
\text { DIS } \\
\text { (MG/L } \\
\text { AS N) }\end{array}$ & $\begin{array}{l}\text { PHOS- } \\
\text { PHORUS } \\
\text { TOTAL } \\
\text { (MG/I } \\
\text { AS P) }\end{array}$ & $\begin{array}{c}\text { PHOS- } \\
\text { PHORUS } \\
\text { DIS- } \\
\text { SOLVED } \\
\text { (MG/L } \\
\text { AS P) }\end{array}$ & $\begin{array}{l}\text { IRON, } \\
\text { TOTAL } \\
\text { RECOV- } \\
\text { ERABLE } \\
\text { (UG/L } \\
\text { AS FE) }\end{array}$ & $\begin{array}{l}\text { MANGA- } \\
\text { NESE, } \\
\text { TOTAL } \\
\text { RECOV- } \\
\text { ERABLE } \\
\text { (UG/L } \\
\text { AS MN) }\end{array}$ & $\begin{array}{l}\text { PHENOLS } \\
\text { TOTAL } \\
\text { (UG/L) }\end{array}$ & $\begin{array}{l}\text { METHY- } \\
\text { LENE } \\
\text { BLUE } \\
\text { ACTIVE } \\
\text { SUB- } \\
\text { STANCE } \\
\text { (MG/L) }\end{array}$ & $\begin{array}{c}\text { CHLOR-A } \\
\text { PHYTO- } \\
\text { PLANK- } \\
\text { TON } \\
\text { CHROMO } \\
\text { FLUOROM } \\
\text { (UG/L) }\end{array}$ & $\begin{array}{c}\text { CHLOR-B } \\
\text { PHYTO- } \\
\text { PLANK- } \\
\text { TON } \\
\text { CHROMO } \\
\text { FLUOROM } \\
\text { (UG/I) }\end{array}$ \\
\hline Nov & & & & & & & & & & \\
\hline MAR $29 . \cdots$ & 0.010 & $<0.20$ & 0.020 & 0.020 & 1100 & 120 & $<1$ & - & -- & -- \\
\hline$\frac{13}{M A Y} \cdot \ldots$ & 0.010 & $<0.20$ & $<0.020$ & $<0.020$ & 1100 & 120 & $<1$ & -- & 0.400 & $<0.100$ \\
\hline $\begin{array}{l}30 \\
\text { AUG }\end{array}$ & 0.050 & $<0.20$ & 0.030 & $<0.020$ & 2500 & 230 & $<1$ & - & 0.200 & $<0.100$ \\
\hline $07 \ldots$ & 0.010 & 0.43 & 0.040 & 0.050 & 950 & 160 & $<1$ & 0.02 & 0.400 & $<0.100$ \\
\hline
\end{tabular}


SURFACE-WATER SITES--ContInued

02433500 TOMBIGBEE RIVER AT BIGBEE, MS

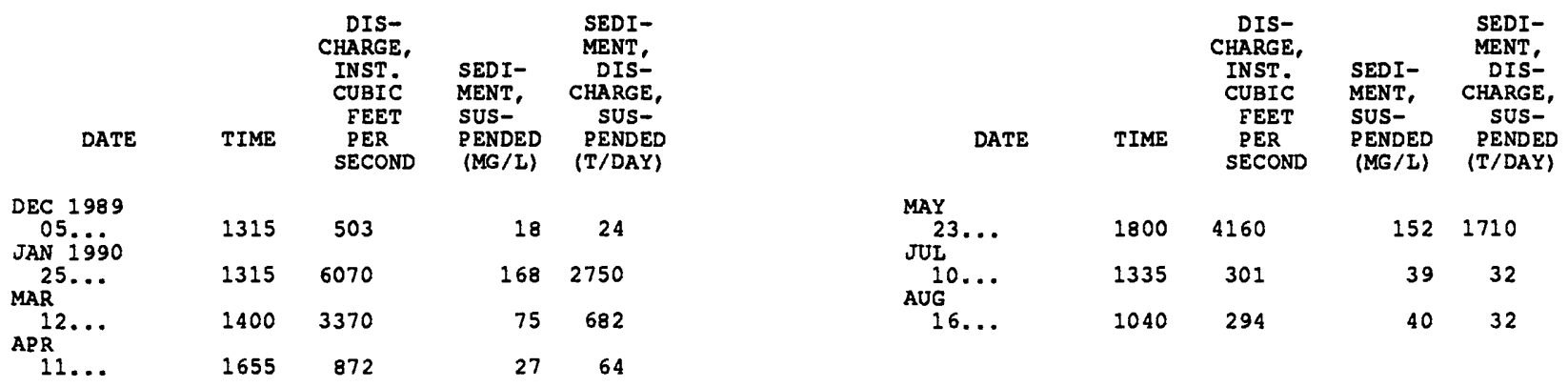

02436500 TOWN CREEK NEAR NETTLETON, MS

\begin{tabular}{|c|c|c|c|c|c|c|c|c|c|c|}
\hline DATE & TIME & $\begin{array}{l}\text { SPE- } \\
\text { CIEIC } \\
\text { CON- } \\
\text { DUCT- } \\
\text { ANCE } \\
\text { (US/CM) }\end{array}$ & $\begin{array}{c}\text { PH } \\
\text { (STAND- } \\
\text { ARD } \\
\text { UNITS) }\end{array}$ & $\begin{array}{c}\text { TEMPER- } \\
\text { ATURE } \\
\text { WATER } \\
\text { (DEG C) }\end{array}$ & $\begin{array}{l}\text { COLOR } \\
\text { (PLAT- } \\
\text { INUM- } \\
\text { COBALT } \\
\text { UNITS) }\end{array}$ & $\begin{array}{l}\text { TUR- } \\
\text { BID- } \\
\text { ITY } \\
\text { (NTU) }\end{array}$ & $\begin{array}{c}\text { TRANS- } \\
\text { PAR- } \\
\text { ENCY } \\
\text { (SECCHI } \\
\text { DISK) } \\
\text { (IN) }\end{array}$ & $\begin{array}{c}\text { OXYGEN, } \\
\text { DIS- } \\
\text { SOLVED } \\
\text { (MG/L) }\end{array}$ & $\begin{array}{l}\text { OXYGEN, } \\
\text { DIS- } \\
\text { SOLVED } \\
\text { (PER- } \\
\text { CENT } \\
\text { SATUR- } \\
\text { ATION) }\end{array}$ & $\begin{array}{c}\text { OXYGEN } \\
\text { DEMAND, } \\
\text { BIO- } \\
\text { CHEM- } \\
\text { ICAL, } \\
5 \text { DAY } \\
\text { (MG/L) }\end{array}$ \\
\hline \multirow{4}{*}{$\begin{array}{l}\text { NOV } 1989 \\
29 . . \\
\text { MAR } 1990 \\
13 \ldots \\
\text { MAY } \ldots \\
30 \ldots \\
\text { AUG } \\
\quad 08 \ldots\end{array}$} & 1010 & 280 & 7.9 & 9.5 & 20 & 21 & 19.5 & 10.6 & 92 & 2.5 \\
\hline & 1540 & 227 & 7.6 & 20.5 & 20 & 25 & 12.5 & 8.7 & 98 & 1.5 \\
\hline & 1505 & 260 & 7.8 & 25.0 & 10 & 26 & 14.5 & 8.4 & 103 & 2.3 \\
\hline & 0740 & 339 & 7.8 & 22.0 & 10 & 2.0 & 9.50 & 7.8 & 90 & 0.6 \\
\hline DATE & $\begin{array}{l}\text { COLI- } \\
\text { FORM, } \\
\text { FECAL, } \\
0.7 \\
\text { UM-MF } \\
\text { (COLS.' } \\
100 \mathrm{ML} \text { ) }\end{array}$ & $\begin{array}{l}\text { CALCIUM } \\
\text { DIS- } \\
\text { SOLVED } \\
\text { (MG/L } \\
\text { AS CA) }\end{array}$ & $\begin{array}{l}\text { MAGNE- } \\
\text { SIUM, } \\
\text { DIS- } \\
\text { SOIVED } \\
\text { (MG/L } \\
\text { AS MG) }\end{array}$ & $\begin{array}{c}\text { ALKA- } \\
\text { LINITY } \\
\text { LAB } \\
\text { (MG/I } \\
\text { AS } \\
\text { CACO3) }\end{array}$ & $\begin{array}{l}\text { SULFATE } \\
\text { DIS- } \\
\text { SOLVED } \\
\text { (MG/I } \\
\text { AS SO4) }\end{array}$ & $\begin{array}{l}\text { CHLO- } \\
\text { RIDE, } \\
\text { DIS- } \\
\text { SOLVED } \\
\text { (MG/L } \\
\text { AS CL) }\end{array}$ & $\begin{array}{l}\text { FLUO- } \\
\text { RIDE, } \\
\text { DIS- } \\
\text { SOLVED } \\
\text { (MG/I } \\
\text { AS F) }\end{array}$ & $\begin{array}{l}\text { SOLIDS, } \\
\text { RESIDUE } \\
\text { AT } 180 \\
\text { DEG. C } \\
\text { DIS- } \\
\text { SOLVED } \\
\text { (MG/L) }\end{array}$ & $\begin{array}{l}\text { RESIDUE } \\
\text { TOTAL } \\
\text { AT } 105 \\
\text { DEG. C, } \\
\text { SUS- } \\
\text { PENDED } \\
\text { (MG/L) }\end{array}$ & $\begin{array}{c}\text { NITRO- } \\
\text { GEN, } \\
\text { NO2+NO3 } \\
\text { DIS- } \\
\text { SOLVED } \\
\text { (MG/L } \\
\text { AS N) }\end{array}$ \\
\hline NOV & & & & & & & & & & \\
\hline $\begin{array}{l}29 \ldots \\
\text { MAR }\end{array}$ & K1900 & 41 & 2.2 & 96 & 21 & 12 & 0.20 & 156 & 36 & 0.490 \\
\hline$\stackrel{13}{M A Y}$ & 1100 & 37 & 1.7 & 85 & 14 & 5.9 & 0.10 & 134 & 102 & 0.280 \\
\hline${ }_{A \cup G}^{30} \cdots$ & K5000 & 43 & 2.0 & 99 & 16 & 10 & 0.20 & 158 & 69 & 0.710 \\
\hline $08 \ldots$ & 240 & 45 & 3.1 & 109 & 20 & 24 & 0.20 & 197 & 10 & 0.760 \\
\hline DATE & $\begin{array}{l}\text { NITRO- } \\
\text { GEN, } \\
\text { AMMONIA } \\
\text { DIS- } \\
\text { SOLVED } \\
\text { (MG/L } \\
\text { AS N) }\end{array}$ & $\begin{array}{l}\text { NITRO- } \\
\text { GEN, AM- } \\
\text { MONIA + } \\
\text { ORGANIC } \\
\text { DIS . } \\
\text { (MG/L } \\
\text { AS N) }\end{array}$ & $\begin{array}{c}\text { PHOS- } \\
\text { PHORUS } \\
\text { TOTAL } \\
\text { (MG/L } \\
\text { AS P) }\end{array}$ & $\begin{array}{l}\text { PHOS- } \\
\text { PHORUS } \\
\text { DIS- } \\
\text { SOLVED } \\
\text { (MG/I } \\
\text { AS P) }\end{array}$ & $\begin{array}{l}\text { IRON, } \\
\text { TOTAL } \\
\text { RECOV- } \\
\text { ERABLE } \\
\text { (UG/L } \\
\text { AS EE) }\end{array}$ & $\begin{array}{l}\text { MANGA- } \\
\text { NESE, } \\
\text { TOTAL } \\
\text { RECOV- } \\
\text { ERABLE } \\
\text { (UG/L } \\
\text { AS MN) }\end{array}$ & $\begin{array}{c}\text { PHENOLS } \\
\text { TOTAL } \\
\text { (UG/L) }\end{array}$ & $\begin{array}{l}\text { METHY- } \\
\text { LENE } \\
\text { BLUE } \\
\text { ACTIVE } \\
\text { SUB- } \\
\text { STANCE } \\
\text { (MG/L) }\end{array}$ & $\begin{array}{c}\text { CHLOR-A } \\
\text { PHYTO- } \\
\text { PLANK- } \\
\text { TON } \\
\text { CHROMO } \\
\text { FLUOROM } \\
\text { (UG /L) }\end{array}$ & $\begin{array}{c}\text { CHLOR-B } \\
\text { PHYTO- } \\
\text { PIANK- } \\
\text { TON } \\
\text { CHROMO } \\
\text { FLUOROM } \\
\text { (UG/L) }\end{array}$ \\
\hline NoV & & & & & & & & & & \\
\hline $\operatorname{MAR}^{29 \ldots}$ & 0.080 & 0.46 & 0.200 & 0.130 & 1400 & 80 & $<1$ & - & 0.600 & $<0: 100$ \\
\hline $\operatorname{MAY}^{13} \ldots$ & 0.060 & 0.35 & 0.130 & 0.030 & 4300 & 80 & $<1$ & - & 1.90 & 0.200 \\
\hline AUG $30 \ldots$ & $<0.010$ & $<0.20$ & 0.190 & 0.090 & 2100 & 110 & $<1$ & - & 1.80 & 0.400 \\
\hline $08 \ldots$ & 0.030 & 0.44 & 0.240 & 0.180 & 420 & 70 & $<1$ & 0.07 & 7.60 & 1.70 \\
\hline
\end{tabular}


SURFACE-WATER SITES--Continued

02436500 TOWN CREEK NEAR NETTLETON, MS--ContInued

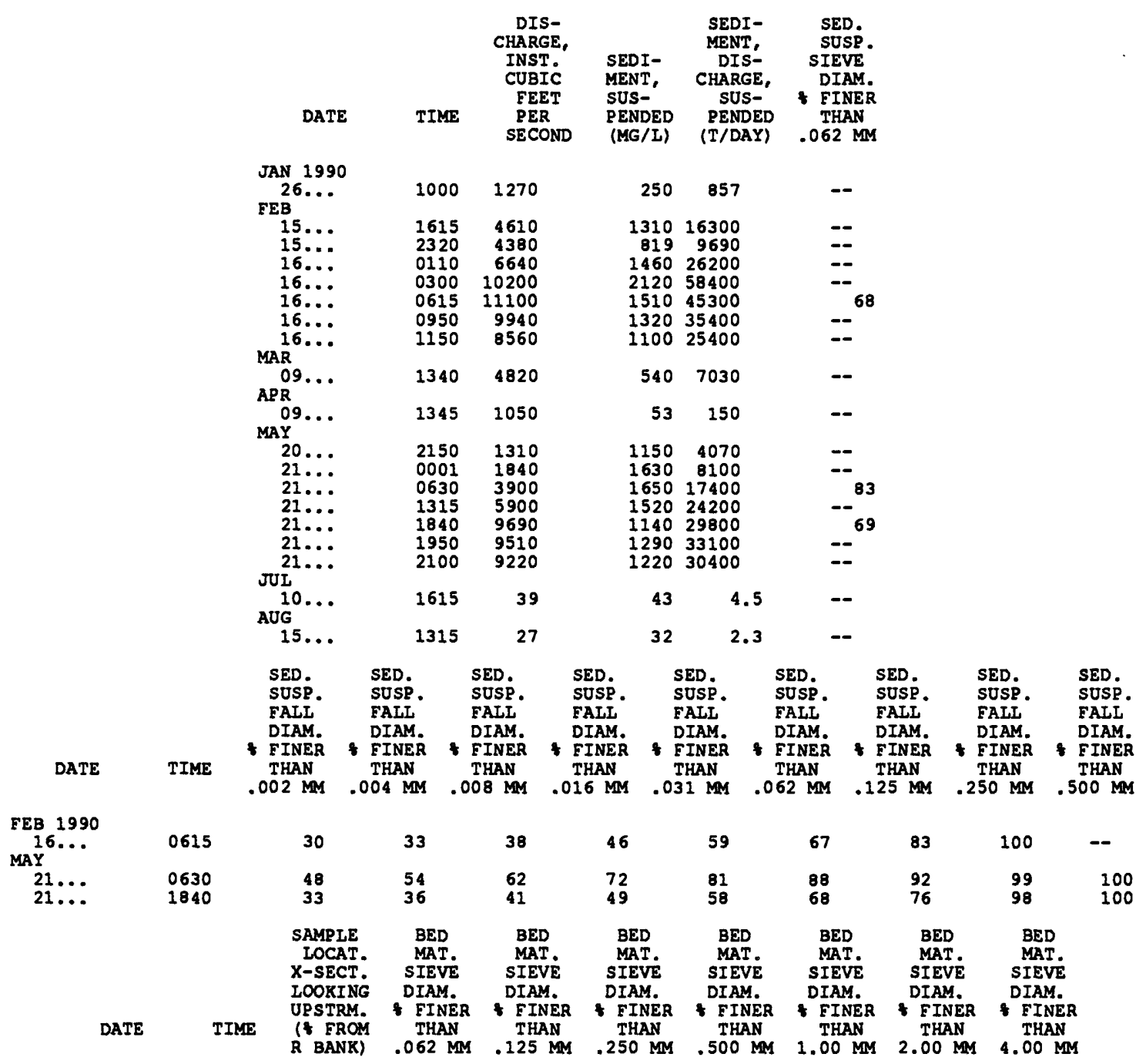

$\begin{array}{crrrrrrrrr}\text { FEB } 1990 & & & & & & \\ 16 \ldots & 0617 & 25.0 & 1 & 2 & 34 & 99 & 100 & -- & -- \\ 16 \ldots & 0619 & 50.0 & 1 & 2 & 25 & 89 & 100 & -- & -- \\ 16 \ldots & 0621 & 75.0 & 1 & 1 & 7 & 36 & 98 & -- & -- \\ \text { MAY } & 1842 & 25.0 & 1 & 2 & 32 & 98 & 100 & -- & -- \\ 21 \ldots & 1844 & 50.0 & 1 & 2 & 16 & 77 & 98 & 100 & -- \\ 21 \ldots & 1846 & 75.0 & <1 & 2 & 14 & 57 & 92 & 96\end{array}$

02437000 TOMBIGBEE RIVER NEAR AMORY, MS

\begin{tabular}{|c|c|c|c|c|c|c|c|c|c|}
\hline DATE & TIME & $\begin{array}{c}\text { DIS- } \\
\text { CHARGE, } \\
\text { INST. } \\
\text { CUBIC } \\
\text { EEET } \\
\text { PER } \\
\text { SECOND }\end{array}$ & $\begin{array}{l}\text { SEDI- } \\
\text { MENT, } \\
\text { SUS- } \\
\text { PENDED } \\
\text { (MG/L) }\end{array}$ & $\begin{array}{c}\text { SEDI- } \\
\text { MENT, } \\
\text { DIS- } \\
\text { CHARGE, } \\
\text { SUS- } \\
\text { PENDED } \\
\text { (T/DAY) }\end{array}$ & DATE & TIME & $\begin{array}{l}\text { DIS- } \\
\text { CHARGE, } \\
\text { INST. } \\
\text { CUBIC } \\
\text { FEET } \\
\text { PER } \\
\text { SECOND }\end{array}$ & $\begin{array}{l}\text { SEDI- } \\
\text { MENT, } \\
\text { SUS- } \\
\text { QENDED } \\
\text { (MG/L) }\end{array}$ & $\begin{array}{c}\text { SEDI- } \\
\text { MENT, } \\
\text { DIS- } \\
\text { CHARGE, } \\
\text { SUS- } \\
\text { PENDED } \\
\text { (T/DAY) }\end{array}$ \\
\hline $\begin{array}{c}\text { JAN } 1990 \\
25 \ldots \\
\text { MAR } \\
13 \ldots\end{array}$ & 1700 & 8440 & 264 & 6020 & $\begin{array}{l}\text { MAY } \\
\quad 23 \ldots\end{array}$ & 1340 & 5870 & 213 & 3380 \\
\hline
\end{tabular}


SURFACE-WATER SITES--Cont 1 nued

02437500 TOMBIGBEE RIVER AT ABERDEEN, MS

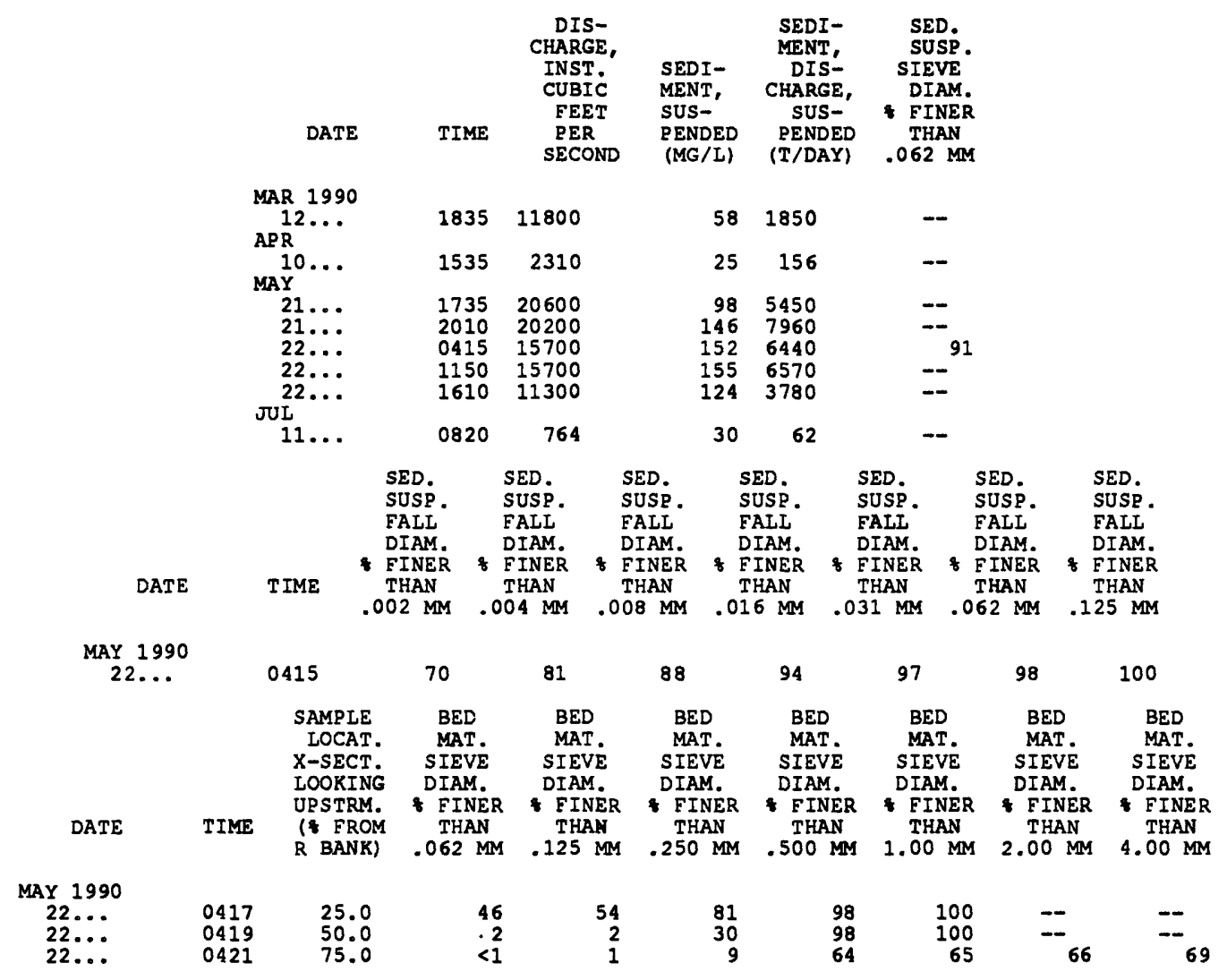

333927088304935 TTW COLUMBUS LAKE BUTTAHATCHEE RIVER BEND SR 26A

\begin{tabular}{|c|c|c|c|c|c|c|c|c|c|}
\hline & DATE & TIME & $\begin{array}{l}\text { SAM- } \\
\text { PLING } \\
\text { DEPTH } \\
\text { (FEET) }\end{array}$ & $\begin{array}{l}\text { SRE- } \\
\text { CIFIC } \\
\text { CON- } \\
\text { DUCT- } \\
\text { ANCE } \\
\text { (US/CM) }\end{array}$ & $\begin{array}{c}\text { PH } \\
\text { (STAND- } \\
\text { ARD } \\
\text { UNITS) }\end{array}$ & $\begin{array}{c}\text { TEMPER- } \\
\text { ATURE } \\
\text { WATER } \\
\text { (DEG C) }\end{array}$ & $\begin{array}{c}\text { OXYGEN, } \\
\text { DIS- } \\
\text { SOLVED } \\
\text { (MG/L) }\end{array}$ & $\begin{array}{c}\text { OXYGEN, } \\
\text { DIS- } \\
\text { SOLVED } \\
\text { (EER- } \\
\text { CENT } \\
\text { SATUR- } \\
\text { ATION) }\end{array}$ & \\
\hline \multirow{3}{*}{\multicolumn{2}{|c|}{$\begin{array}{c}\text { NOV } 1989 \\
30 \ldots \\
30 \ldots \\
30 \ldots \\
30 \ldots \\
\text { MAR } 1990 \\
14 \ldots \ldots \\
14 \ldots \ldots \\
14 \ldots \\
\text { AUG } \\
08 \ldots \\
08 \ldots \\
08 \ldots \\
08 \ldots\end{array}$}} & $\begin{array}{l}1126 \\
1127 \\
1128 \\
1129\end{array}$ & $\begin{array}{c}1.00 \\
5.00 \\
10.0 \\
12.0\end{array}$ & $\begin{array}{l}138 \\
139 \\
137 \\
139\end{array}$ & $\begin{array}{l}7.3 \\
7.3 \\
7.3 \\
7.3\end{array}$ & $\begin{array}{l}11.0 \\
11.0 \\
11.0 \\
11.0\end{array}$ & $\begin{array}{l}9.8 \\
9.8 \\
9.7 \\
9.7\end{array}$ & $\begin{array}{l}88 \\
88 \\
87 \\
87\end{array}$ & \\
\hline & & $\begin{array}{l}1431 \\
1432 \\
1433\end{array}$ & $\begin{array}{l}1.00 \\
5.00 \\
8.00\end{array}$ & $\begin{array}{l}125 \\
125 \\
125\end{array}$ & $\begin{array}{l}7.2 \\
7.2 \\
7.2\end{array}$ & $\begin{array}{l}18.0 \\
18.0 \\
18.0\end{array}$ & $\begin{array}{l}9.4 \\
9.2 \\
9.2\end{array}$ & $\begin{array}{r}101 \\
98 \\
98\end{array}$ & \\
\hline & & $\begin{array}{l}1501 \\
1502 \\
1503 \\
1504\end{array}$ & $\begin{array}{r}1.00 \\
5.00 \\
10.0 \\
12.0\end{array}$ & $\begin{array}{l}210 \\
210 \\
210 \\
212\end{array}$ & $\begin{array}{l}7.3 \\
7.0 \\
6.9 \\
6.8\end{array}$ & $\begin{array}{l}30.0 \\
28.5 \\
28.5 \\
28.0\end{array}$ & $\begin{array}{l}7.3 \\
6.0 \\
5.0 \\
4.4\end{array}$ & $\begin{array}{l}97 \\
78 \\
65 \\
57\end{array}$ & \\
\hline DATE & TIME & $\begin{array}{l}\text { COLOR } \\
\text { (PLAT- } \\
\text { INUM- } \\
\text { COBALT } \\
\text { UNITS) }\end{array}$ & $\begin{array}{l}\text { TUR- } \\
\text { BID- } \\
\text { ITY } \\
\text { (NTU) }\end{array}$ & $\begin{array}{c}\text { TRANS- } \\
\text { PAR- } \\
\text { ENCY } \\
\text { (SECCHI } \\
\text { DISK) } \\
\text { (IN) }\end{array}$ & $\begin{array}{l}\text { OXYGEN } \\
\text { DEMAND, } \\
\text { BIO- } \\
\text { CHEM- } \\
\text { ICAL, } \\
5 \text { DAY } \\
\text { (MG/L) }\end{array}$ & $\begin{array}{l}\text { COLI- } \\
\text { FORM, } \\
\text { FECAL, } \\
0.7 \\
\text { UM-MF } \\
\text { (COLS. } \\
100 \mathrm{ML} \text { ) }\end{array}$ & $\begin{array}{l}\text { CALCIUM } \\
\text { DIS- } \\
\text { SOLVED } \\
\text { (MG/L } \\
\text { AS CA) }\end{array}$ & $\begin{array}{c}\text { MAGNE- } \\
\text { SIUM, } \\
\text { DIS- } \\
\text { SOLVED } \\
\text { (MG/L } \\
\text { AS MG) }\end{array}$ & $\begin{array}{c}\text { ALKA- } \\
\text { LINITY } \\
\text { IAB } \\
\text { (MG/L } \\
\text { AS } \\
\text { CACO3) }\end{array}$ \\
\hline \multirow{4}{*}{$\begin{array}{l}\text { NOV } 1989 \\
30 \ldots \\
\text { MAR } 1990 \\
14 \ldots \\
\text { MAY } \\
31 \ldots \\
\text { AUG } \\
\text { O8.... }\end{array}$} & 1125 & 60 & 33 & 12.0 & 1.7 & 150 & 17 & 1.4 & 31 \\
\hline & 1430 & 60 & 35 & 14.0 & 0.9 & 130 & 18 & 1.2 & 35 \\
\hline & 1210 & 120 & 42 & 8.25 & 1.7 & 150 & 15 & 1.0 & 25 \\
\hline & 1500 & 10 & 7.5 & 20.2 & 0.6 & 26 & 25 & 1.8 & 42 \\
\hline
\end{tabular}


SURFACE-WATER SITES--COnt1nued

333927088304935 TTW COLUMBUS LAKE BUTTAHATCHEE RIVER BEND SR 26A--Cont1nued $\begin{array}{cllcllcr} & \text { CHLO- } & \text { FLUO- } & \text { RESIDUE } & \text { TOTAL } & \text { GEN, } & \text { GEN, } & \text { GEN, AM- } \\ \text { SULFATE } & \text { RIDE, } & \text { RIDE, } & \text { AT } 180 & \text { AT 105 } & \text { NO2+NO3 } & \text { AMMONIA } & \text { MONIA } \\ \text { DIS- } & \text { DIS- } & \text { DIS- } & \text { DEG.C } & \text { DEG. C, } & \text { DIS- } & \text { DIS- } & \text { ORGANIC } \\ \text { SOLVED } & \text { SOLVED } & \text { SOLVED } & \text { DIS- } & \text { SUS- } & \text { SOLVED } & \text { SOLVED } & \text { DIS. } \\ \text { (MG/L } & \text { (MG/L } & \text { (MG/L } & \text { SOLVED } & \text { PENDED } & \text { (MG/L } & \text { (MG/L } & \text { (MG/L } \\ \text { AS SO4) } & \text { AS CL) } & \text { AS F) } & \text { (MG/L) } & \text { (MG/L) } & \text { AS N) } & \text { AS N) } & \text { AS N) }\end{array}$

\begin{tabular}{|c|c|c|c|c|c|c|c|c|}
\hline $\begin{array}{l}\text { NOV } \\
30 \ldots \\
\text { MAR }\end{array}$ & 12 & 11 & 0.20 & 85 & 37 & 0.140 & 0.070 & 0.38 \\
\hline $\operatorname{MAY}^{14} \ldots$ & 9.5 & 5.5 & 0.06 & 76 & 46 & 0.190 & 0.050 & 0.59 \\
\hline $\begin{array}{l}31 \ldots \\
\text { AUG }\end{array}$ & 7.6 & 11 & 0.10 & 79 & 43 & 0.290 & 0.050 & 0.22 \\
\hline $08 \ldots$ & 16 & 26 & 0.10 & 132 & 16 & $<0.020$ & 0.010 & 0.33 \\
\hline DATE & $\begin{array}{l}\text { PHOS- } \\
\text { PHORUS } \\
\text { TOTAL } \\
\text { (MG/I } \\
\text { AS P) }\end{array}$ & $\begin{array}{c}\text { PHOS- } \\
\text { PHORUS } \\
\text { DIS- } \\
\text { SOLVED } \\
\text { (MG / } \\
\text { AS P) }\end{array}$ & $\begin{array}{l}\text { IRON, } \\
\text { TOTAL } \\
\text { RECOV- } \\
\text { ERABLE } \\
\text { (UG/L } \\
\text { AS FE) }\end{array}$ & $\begin{array}{l}\text { MANGA- } \\
\text { NESE, } \\
\text { TOTAL } \\
\text { RECOV- } \\
\text { ERABLE } \\
\text { (UG/L } \\
\text { AS MN) }\end{array}$ & $\begin{array}{l}\text { PHENOIS } \\
\text { TOTAL } \\
\text { (UG/I) }\end{array}$ & $\begin{array}{l}\text { METHY- } \\
\text { LENE } \\
\text { BLUE } \\
\text { ACTIVE } \\
\text { SUB- } \\
\text { STANCE } \\
\text { (MG/L) }\end{array}$ & $\begin{array}{l}\text { CHLOR-A } \\
\text { PHYTO- } \\
\text { PLANK- } \\
\text { TON } \\
\text { CHROMO } \\
\text { FLUOROM } \\
\text { (UG /L) }\end{array}$ & $\begin{array}{l}\text { CHLOR-B } \\
\text { P HYTO- } \\
\text { P LANK- } \\
\text { TON } \\
\text { CHROMO } \\
\text { FLUOROM } \\
\text { (UG/L) }\end{array}$ \\
\hline
\end{tabular}

\begin{tabular}{|c|c|c|c|c|c|c|c|c|}
\hline $\begin{array}{l}\text { NOV } \\
30 \ldots \\
M A R\end{array}$ & 0.080 & 0.020 & 1900 & 150 & $<1$ & -- & 1.40 & 0.100 \\
\hline${ }_{\mathrm{MAY}}^{14} \ldots$ & 0.060 & 0.020 & 2500 & 110 & $<1$ & -- & 2.50 & 0.200 \\
\hline AUG $31 \ldots$ & 0.080 & $<0.020$ & 2200 & 110 & $<1$ & -- & 1.30 & $<0.100$ \\
\hline $08 \ldots$ & 0.060 & 0.030 & 590 & 230 & $<1$ & 0.05 & 2.20 & 0.500 \\
\hline
\end{tabular}

02439400 BUTTAHATCHEE RIVER NEAR ABERDEEN, MS

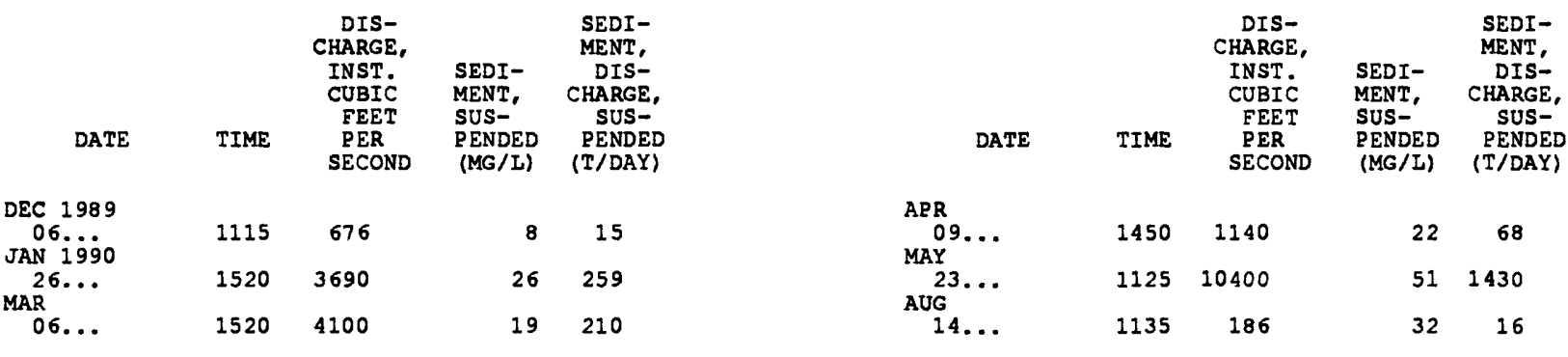

02439600 BUTTAHATCHEE RIVER NEAR KOLOLA SPRINGS, MS

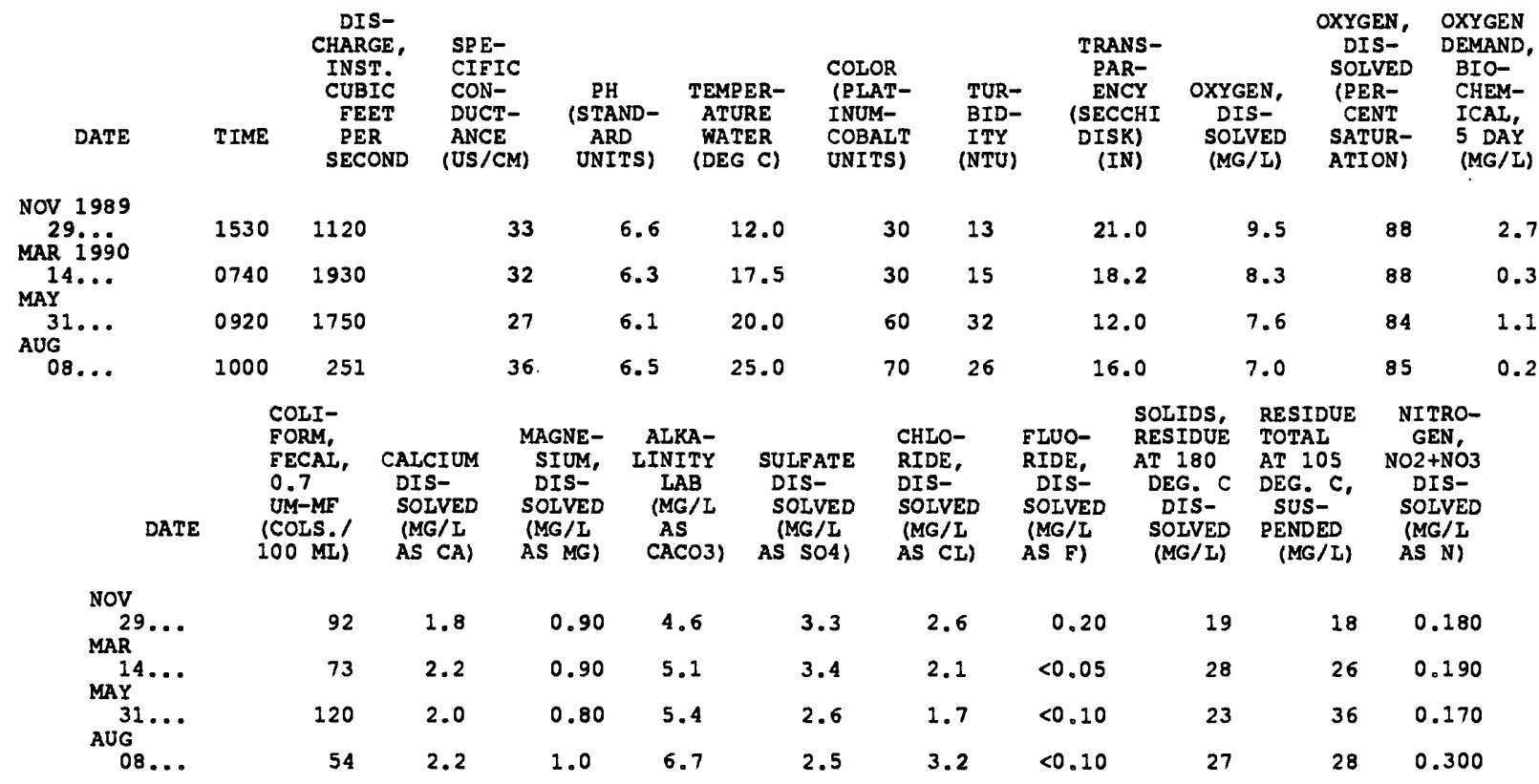


SURFACE-WATER SITES--Cont1 nued

02439600 BUTTAHATCHEE RIVER NEAR KOLOLA SPRINGS, MS--Continued

\begin{tabular}{|c|c|c|c|c|c|c|c|c|c|c|}
\hline DATE & $\begin{array}{l}\text { NITRO- } \\
\text { GEN, } \\
\text { AMMONIA } \\
\text { DIS- } \\
\text { SOLVED } \\
\text { (MG/L } \\
\text { AS N) }\end{array}$ & $\begin{array}{l}\text { NITRO- } \\
\text { GEN,AM- } \\
\text { MONIA + } \\
\text { ORGANIC } \\
\text { DIS. } \\
\text { (MG/L } \\
\text { AS N) }\end{array}$ & $\begin{array}{l}\text { PHOS- } \\
\text { PHORUS } \\
\text { TOTAL } \\
\text { (MG/L } \\
\text { AS P) }\end{array}$ & $\begin{array}{c}\text { PHOS- } \\
\text { PHORUS } \\
\text { DIS- } \\
\text { SOLVED } \\
\text { (MG/I } \\
\text { AS P) }\end{array}$ & $\begin{array}{l}\text { IRON, } \\
\text { TOTAL } \\
\text { RECOV- } \\
\text { ERABLE } \\
\text { (UG/I } \\
\text { AS FE) }\end{array}$ & $\begin{array}{l}\text { MANGA- } \\
\text { NESE, } \\
\text { TOTAL } \\
\text { RECOV- } \\
\text { ERABLE } \\
\text { (UG/L } \\
\text { AS MN) }\end{array}$ & $\begin{array}{l}\text { PHENOLS } \\
\text { TOTAL } \\
\text { (UG/L) }\end{array}$ & $\begin{array}{l}\text { METHY- } \\
\text { LENE } \\
\text { BLUE } \\
\text { ACTIVE } \\
\text { SUB- } \\
\text { STANCE } \\
\text { (MG/L) }\end{array}$ & $\begin{array}{c}\text { CHLOR-A } \\
\text { P HYTO- } \\
\text { PIANK- } \\
\text { TON } \\
\text { CHROMO } \\
\text { FLUOROM } \\
\text { (UG/L) }\end{array}$ & $\begin{array}{c}\text { CHLOR-B } \\
\text { PHYTO- } \\
\text { PLANK- } \\
\text { TON } \\
\text { CHROMO } \\
\text { FLUOROM } \\
\text { (UG/L) }\end{array}$ \\
\hline$\cdots$ & 0.010 & $<0.20$ & 0.040 & 0.020 & 1200 & 140 & $<1$ & -- & $<0.100$ & $<0.100$ \\
\hline$\cdots$ & 0.020 & $<0.20$ & 0.020 & 0.020 & 1300 & 130 & $<1$ & - & 0.300 & $<0.100$ \\
\hline$\ldots$ & 0.060 & $<0.20$ & 0.040 & $<0.020$ & 2200 & 150 & $<1$ & -- & 0.400 & $<0.100$ \\
\hline 3... & 0.010 & 0.75 & 0.050 & 0.030 & 1700 & 200 & $<1$ & 0.06 & 0.600 & $<0.100$ \\
\hline
\end{tabular}

02441000 TIBBEE CREEK NEAR TIBBEE, MS

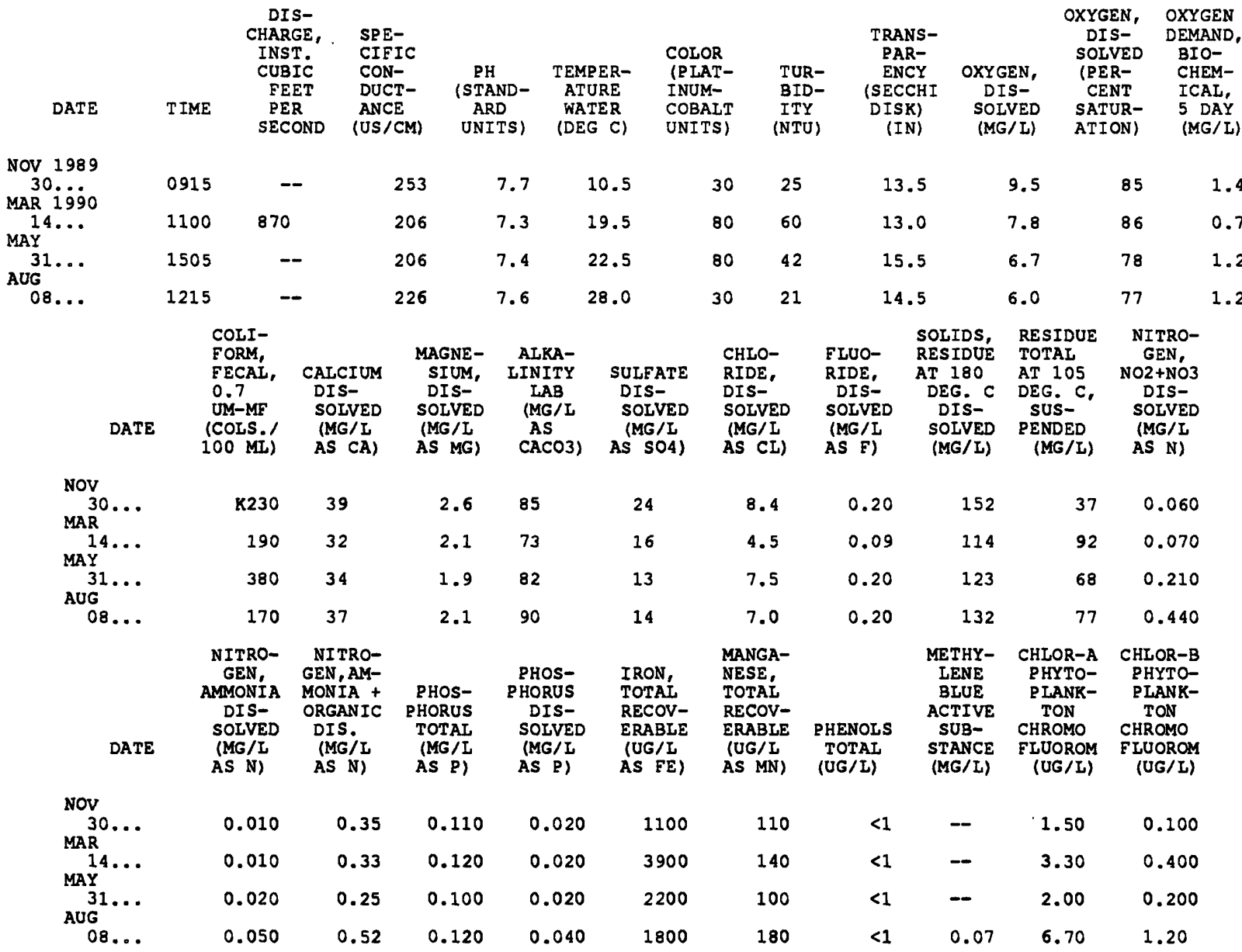


02441400 TOMBIGBEE RIVER NEAR COLUMBUS, MS

\begin{tabular}{|c|c|c|c|c|c|c|c|c|c|}
\hline & DATE & TIME & $\begin{array}{l}\text { SAM- } \\
\text { PLING } \\
\text { DEPTH } \\
\text { (FEET) }\end{array}$ & $\begin{array}{l}\text { SPE- } \\
\text { CIFIC } \\
\text { CON- } \\
\text { DUCT- } \\
\text { ANCE } \\
\text { (US/CM) }\end{array}$ & $\begin{array}{c}\text { PH } \\
\text { (STAND- } \\
\text { ARD } \\
\text { UNITS) }\end{array}$ & $\begin{array}{c}\text { TEMPER- } \\
\text { ATURE } \\
\text { WATER } \\
\text { (DEG C) }\end{array}$ & $\begin{array}{c}\text { OXYGEN, } \\
\text { DIS- } \\
\text { SOLVED } \\
\text { (MG/L) }\end{array}$ & $\begin{array}{c}\text { OXYGEN, } \\
\text { DIS- } \\
\text { SOLVED } \\
\text { (PER- } \\
\text { CENT } \\
\text { SATUR- } \\
\text { ATION) }\end{array}$ & \\
\hline & $\begin{array}{c}\text { NOV } 1989 \\
30 \ldots \\
30 \ldots \\
30 \ldots \\
30 \ldots\end{array}$ & $\begin{array}{l}1446 \\
1447 \\
1448 \\
1449\end{array}$ & $\begin{array}{l}1.00 \\
5.00 \\
10.0 \\
13.0\end{array}$ & $\begin{array}{l}119 \\
119 \\
117 \\
117\end{array}$ & $\begin{array}{l}7.5 \\
7.4 \\
7.4 \\
7.4\end{array}$ & $\begin{array}{l}11.0 \\
10.5 \\
10.5 \\
10.5\end{array}$ & $\begin{array}{l}11.0 \\
10.9 \\
10.9 \\
10.9\end{array}$ & $\begin{array}{l}99 \\
97 \\
97 \\
97\end{array}$ & \\
\hline & $\begin{array}{c}\text { MAR } 1990 \\
15 \ldots \\
15 \ldots \\
15 \ldots \\
15 \ldots \\
\text { JuN }\end{array}$ & $\begin{array}{l}0911 \\
0912 \\
0913 \\
0914\end{array}$ & $\begin{array}{l}1.00 \\
5.00 \\
10.0 \\
15.0\end{array}$ & $\begin{array}{l}103 \\
103 \\
102 \\
102\end{array}$ & $\begin{array}{l}7.2 \\
7.2 \\
7.2 \\
7.2\end{array}$ & $\begin{array}{l}18.0 \\
18.0 \\
18.0 \\
18.0\end{array}$ & $\begin{array}{l}10.8 \\
10.9 \\
11.0 \\
11.0\end{array}$ & $\begin{array}{l}115 \\
116 \\
117 \\
117\end{array}$ & \\
\hline & $\begin{array}{r}01 \ldots \\
01 \ldots \\
01 \ldots \\
01 \ldots \\
01 \ldots\end{array}$ & $\begin{array}{l}0916 \\
0917 \\
0918 \\
0919 \\
0920\end{array}$ & $\begin{array}{l}1.00 \\
5.00 \\
10.0 \\
15.0 \\
18.0\end{array}$ & $\begin{array}{l}73 \\
73 \\
72 \\
72 \\
71\end{array}$ & $\begin{array}{l}6.8 \\
6.8 \\
6.8 \\
6.8 \\
6.8\end{array}$ & $\begin{array}{l}22.5 \\
22.5 \\
22.5 \\
22.5 \\
22.5\end{array}$ & $\begin{array}{l}9.2 \\
9.2 \\
9.2 \\
9.2 \\
9.3\end{array}$ & $\begin{array}{l}107 \\
107 \\
107 \\
107 \\
108\end{array}$ & \\
\hline & $\begin{array}{l}09 . . . \\
09 . \ldots \\
09 . \ldots \\
09 . .\end{array}$ & $\begin{array}{l}0901 \\
0902 \\
0903 \\
0904\end{array}$ & $\begin{array}{c}1.00 \\
5.00 \\
10.0 \\
14.0\end{array}$ & $\begin{array}{l}210 \\
201 \\
201 \\
201\end{array}$ & $\begin{array}{l}7.5 \\
7.5 \\
7.5 \\
7.5\end{array}$ & $\begin{array}{l}27.5 \\
27.5 \\
27.5 \\
27.5\end{array}$ & $\begin{array}{l}7.6 \\
7.3 \\
7.2 \\
7.3\end{array}$ & $\begin{array}{l}97 \\
93 \\
92 \\
93\end{array}$ & \\
\hline DATE & TIME & $\begin{array}{c}\text { DIS- } \\
\text { CHARGE, } \\
\text { INST. } \\
\text { CUBIC } \\
\text { FEET } \\
\text { PER } \\
\text { SECOND }\end{array}$ & $\begin{array}{l}\text { COLOR } \\
\text { (PIAT- } \\
\text { INUM- } \\
\text { COBALT } \\
\text { UNITS) }\end{array}$ & $\begin{array}{l}\text { TUR- } \\
\text { BID- } \\
\text { ITY } \\
\text { (NTU) }\end{array}$ & $\begin{array}{c}\text { TRANS- } \\
\text { PAR- } \\
\text { ENCY } \\
\text { (SECCHI } \\
\text { DISK) } \\
\text { (IN) }\end{array}$ & $\begin{array}{l}\text { OXYGEN } \\
\text { DEMAND, } \\
\text { BIO- } \\
\text { CHEM- } \\
\text { ICAL, } \\
5 \text { DAY } \\
\text { (MG/L) }\end{array}$ & $\begin{array}{l}\text { COLI- } \\
\text { FORM, } \\
\text { FECAL, } \\
\text { O.7 } \\
\text { UM-MF } \\
\text { (COLS.' } \\
100 \mathrm{ML} \text { ) }\end{array}$ & $\begin{array}{l}\text { CALCIUM } \\
\text { DIS- } \\
\text { SOLVED } \\
\text { (MG/I } \\
\text { AS CA) }\end{array}$ & $\begin{array}{l}\text { MAGNE- } \\
\text { SIUM, } \\
\text { DIS- } \\
\text { SOLVED } \\
\text { (MG/L } \\
\text { AS MG) }\end{array}$ \\
\hline $\begin{array}{l}V \\
V \\
30 \\
R\end{array}$ & 1445 & 3310 & 60 & 28 & 13.5 & 2.0 & 120 & 14 & 1.3 \\
\hline $15 \ldots$ & 0910 & 15800 & 60 & 33 & 10.5 & 0.9 & 110 & 14 & 1.1 \\
\hline${ }_{j G}^{01} \cdots$ & 0915 & 4800 & 80 & 50 & 10.3 & 1.5 & 140 & 9.3 & 0.90 \\
\hline $09 . .$. & 0900 & 702 & 10 & 5.4 & 24.5 & 1.2 & K5 & 23 & 1.7 \\
\hline DATE & $\begin{array}{c}\text { ALKA- } \\
\text { LINITY } \\
\text { IAB } \\
\text { (MG/L } \\
\text { AS } \\
\text { CACO3) }\end{array}$ & $\begin{array}{l}\text { SULEATE } \\
\text { DIS- } \\
\text { SOLVED } \\
\text { (MG/L } \\
\text { AS SO4) }\end{array}$ & $\begin{array}{l}\text { CHLO- } \\
\text { RIDE, } \\
\text { DIS- } \\
\text { SOIVED } \\
\text { (MG/L } \\
\text { AS CL) }\end{array}$ & $\begin{array}{l}\text { FLUO- } \\
\text { RIDE, } \\
\text { DIS- } \\
\text { SOLVED } \\
\text { (MG/L } \\
\text { AS F) }\end{array}$ & $\begin{array}{l}\text { SOLIDS, } \\
\text { RESIDUE } \\
\text { AT } 180 \\
\text { DEG. C } \\
\text { DIS- } \\
\text { SOLVED } \\
\text { (MG/L) }\end{array}$ & $\begin{array}{l}\text { RESIDUE } \\
\text { TOTAL } \\
\text { AT } 105 \\
\text { DEG. C, } \\
\text { SUS- } \\
\text { PENDED } \\
\text { (MG/L) }\end{array}$ & $\begin{array}{c}\text { NITRO- } \\
\text { GEN, } \\
\text { NO2+NO3 } \\
\text { DIS- } \\
\text { SOLVED } \\
\text { (MG/L } \\
\text { AS N) }\end{array}$ & $\begin{array}{l}\text { NITRO- } \\
\text { GEN, } \\
\text { AMMONIA } \\
\text { DIS- } \\
\text { SOLVED } \\
\text { (MG/L } \\
\text { AS N) }\end{array}$ & $\begin{array}{l}\text { NITRO- } \\
\text { GEN, AM- } \\
\text { MONIA + } \\
\text { ORGANIC } \\
\text { DIS. } \\
\text { (MG/L } \\
\text { AS N) }\end{array}$ \\
\hline
\end{tabular}

\begin{tabular}{|c|c|c|c|c|c|c|c|c|c|}
\hline $\begin{array}{l}\text { NOV } \\
30 \ldots \\
\text { MAR } \ldots\end{array}$ & 28 & 9.6 & 8.4 & 0.50 & 71 & 30 & 1.60 & 0.050 & 0.49 \\
\hline $15 \ldots$ & 29 & 7.5 & 4.7 & 0.06 & 61 & 45 & 0.200 & 0.050 & 0.32 \\
\hline $01 \ldots$ & 18 & 5.2 & 6.5 & $<0.10$ & 50 & 54 & 0.250 & 0.040 & 0.27 \\
\hline $09 . .$. & 43 & 14 & 24 & $<0.10$ & 121 & 14 & 0.020 & 0.010 & 0.73 \\
\hline
\end{tabular}


SURFACE-WATER SITES--Cont1nued

332929088273300 TTW ALICEVILLE LAKE ABOVE COLUMBUS BEND

\begin{tabular}{|c|c|c|c|c|c|c|c|c|c|c|c|}
\hline DATE & TIME & $\begin{array}{l}\text { SAMPIE } \\
\text { IOCAT. } \\
\text { X-SECT: } \\
\text { LOOKING } \\
\text { UP STRM. } \\
\text { (\& FROM } \\
\text { R BANK) }\end{array}$ & $\begin{array}{c}\text { BED } \\
\text { MAT. } \\
\text { SIEVE } \\
\text { DIAM. } \\
\text { FINER } \\
\text { THAN } \\
.062 \mathrm{MM}\end{array}$ & $\begin{array}{c}\text { BED } \\
\text { MAT. } \\
\text { SIEVE } \\
\text { DIAM. } \\
\text { \& FINER } \\
\text { THAN } \\
.125 \mathrm{MM}\end{array}$ & $\begin{array}{c}\text { BED } \\
\text { MAT. } \\
\text { SIEVE } \\
\text { DIAM. } \\
\text { FINER } \\
\text { FIHAN } \\
.250 \mathrm{MM}\end{array}$ & $\begin{array}{c}\text { BED } \\
\text { MAT. } \\
\text { SIEVE } \\
\text { DIAM. } \\
\text { \& FINER } \\
\text { THAN } \\
500 \mathrm{MM}\end{array}$ & $\begin{array}{c}\text { BED } \\
\text { MAT. } \\
\text { SIEVE } \\
\text { DIAM. } \\
\text { \& FINER } \\
\text { THAN } \\
1.00 \mathrm{MM}\end{array}$ & $\begin{array}{c}\text { BED } \\
\text { MAT. } \\
\text { SIEVE } \\
\text { DIAM. } \\
\text { \& FINER } \\
\text { THAN } \\
2.00 \mathrm{MM}\end{array}$ & $\begin{array}{c}\text { BED } \\
\text { MAT. } \\
\text { SIEVE } \\
\text { DIAM. } \\
\text { FINER } \\
\text { THAN } \\
\text { T.00 MM }\end{array}$ & $\begin{array}{c}\text { BED } \\
\text { MAT. } \\
\text { SIEVE } \\
\text { DIAM. } \\
\text { \&INER } \\
\text { THAN } \\
8.00 \mathrm{MM}\end{array}$ & $\begin{array}{c}\text { BED } \\
\text { MAT. } \\
\text { SIEVE } \\
\text { DIAM. } \\
\text { \& FINER } \\
\text { THAN } \\
16.0 \mathrm{MM}\end{array}$ \\
\hline AN & & & & & & & & & & & \\
\hline $\begin{array}{l}24 \ldots \\
24 \ldots \\
24 \ldots\end{array}$ & $\begin{array}{l}1250 \\
1300 \\
1310\end{array}$ & $\begin{array}{l}25.0 \\
50.0 \\
75.0\end{array}$ & $\begin{array}{l}0 \\
0 \\
0\end{array}$ & $\begin{array}{l}1 \\
0 \\
1\end{array}$ & $\begin{array}{r}2 \\
4 \\
13\end{array}$ & $\begin{array}{r}10 \\
8 \\
79\end{array}$ & $\begin{array}{r}14 \\
9 \\
84\end{array}$ & $\begin{array}{l}17 \\
10 \\
87\end{array}$ & $\begin{array}{l}22 \\
15 \\
90\end{array}$ & $\begin{array}{l}36 \\
31 \\
95\end{array}$ & $\begin{array}{r}69 \\
72 \\
100\end{array}$ \\
\hline
\end{tabular}

02441498 TOMBIGBEE RIVER COLUMBUS BEND SR 11B AT COLUMBUS, MS

\begin{tabular}{|c|c|c|c|c|c|c|c|c|}
\hline DATE & TIME & $\begin{array}{l}\text { SAM- } \\
\text { PIING } \\
\text { DEPTH } \\
\text { (EEET) }\end{array}$ & $\begin{array}{l}\text { SPE- } \\
\text { CIFIC } \\
\text { CON- } \\
\text { DUCT- } \\
\text { ANCE } \\
\text { (US/CM) }\end{array}$ & $\begin{array}{c}\text { PH } \\
\text { (STAND- } \\
\text { ARD } \\
\text { UNITS) }\end{array}$ & $\begin{array}{c}\text { TEMPER- } \\
\text { ATURE } \\
\text { WATER } \\
\text { (DEG C) }\end{array}$ & $\begin{array}{c}\text { OXYGEN, } \\
\text { DIS- } \\
\text { SOIVED } \\
\text { (MG/L) }\end{array}$ & $\begin{array}{c}\text { OXYGEN, } \\
\text { DIS- } \\
\text { SOLVED } \\
\text { (PER- } \\
\text { CENT } \\
\text { SATUR- } \\
\text { ATION) }\end{array}$ & \\
\hline $\begin{array}{r}\text { NOV } \\
30 \ldots \\
30 \ldots \\
30 \ldots \\
30 \ldots \\
30 \ldots \\
30 \ldots\end{array}$ & $\begin{array}{l}1530 \\
1531 \\
1532 \\
1534 \\
1535 \\
1536\end{array}$ & $\begin{array}{l}-- \\
5.00 \\
10.0 \\
15.0 \\
20.0 \\
23.0\end{array}$ & $\begin{array}{l}107 \\
107 \\
107 \\
107 \\
107 \\
107\end{array}$ & $\begin{array}{l}7.4 \\
7.3 \\
7.3 \\
7.3 \\
7.3 \\
7.3\end{array}$ & $\begin{array}{l}10.5 \\
10.5 \\
10.5 \\
10.5 \\
10.5 \\
10.5\end{array}$ & $\begin{array}{l}11.0 \\
10.9 \\
10.9 \\
10.9 \\
10.8 \\
10.8\end{array}$ & $\begin{array}{l}-- \\
-- \\
-- \\
-- \\
--\end{array}$ & \\
\hline $\begin{array}{r}15 \ldots \\
15 \ldots \\
15 \ldots \\
15 \ldots \\
15 \ldots\end{array}$ & $\begin{array}{l}1001 \\
1002 \\
1003 \\
1004 \\
1005\end{array}$ & $\begin{array}{c}-- \\
5.00 \\
10.0 \\
15.0 \\
17.0\end{array}$ & $\begin{array}{l}111 \\
111 \\
111 \\
112 \\
112\end{array}$ & $\begin{array}{l}7.3 \\
7.3 \\
7.2 \\
7.2 \\
7.2\end{array}$ & $\begin{array}{l}18.0 \\
18.0 \\
18.0 \\
18.0 \\
18.0\end{array}$ & $\begin{array}{r}10.1 \\
9.9 \\
9.9 \\
9.8 \\
9.9\end{array}$ & $\begin{array}{l}108 \\
106 \\
106 \\
104 \\
106\end{array}$ & \\
\hline $\begin{array}{l}01 \ldots \\
01 \ldots \\
01 \ldots \\
01 \ldots \\
01 \ldots \\
01 \ldots \\
\text { AUG }\end{array}$ & $\begin{array}{l}0940 \\
0941 \\
0942 \\
0943 \\
0944 \\
0945\end{array}$ & $\begin{array}{l}-- \\
5.00 \\
10.0 \\
15.0 \\
20.0 \\
25.0\end{array}$ & $\begin{array}{l}73 \\
75 \\
73 \\
73 \\
73 \\
73\end{array}$ & $\begin{array}{l}6.9 \\
6.8 \\
6.8 \\
6.8 \\
6.8 \\
6.8\end{array}$ & $\begin{array}{l}22.5 \\
22.5 \\
22.5 \\
22.5 \\
22.5 \\
22.5\end{array}$ & $\begin{array}{l}8.6 \\
8.6 \\
8.6 \\
8.4 \\
8.5 \\
8.6\end{array}$ & $\begin{array}{l}99 \\
-- \\
-- \\
-- \\
--\end{array}$ & \\
\hline $\begin{array}{l}09 . \ldots \\
09 \ldots . . \\
09 \ldots \\
09 \ldots \\
09 \ldots\end{array}$ & $\begin{array}{l}0940 \\
0941 \\
0942 \\
0943 \\
0944\end{array}$ & $\begin{array}{l}5.00 \\
10.0 \\
15.0 \\
20.0\end{array}$ & $\begin{array}{l}191 \\
192 \\
192 \\
192 \\
192\end{array}$ & $\begin{array}{l}7.0 \\
7.1 \\
7.0 \\
7.0 \\
7.0\end{array}$ & $\begin{array}{l}28.5 \\
28.0 \\
28.0 \\
28.0 \\
28.0\end{array}$ & $\begin{array}{l}6.0 \\
5.8 \\
5.7 \\
5.7 \\
5.6\end{array}$ & $\begin{array}{l}77 \\
-- \\
-- \\
--\end{array}$ & \\
\hline TIME & $\begin{array}{l}\text { COLOR } \\
\text { (PIAT- } \\
\text { INUM- } \\
\text { COBALT } \\
\text { UNITS) }\end{array}$ & $\begin{array}{l}\text { TUR- } \\
\text { BID- } \\
\text { ITY } \\
\text { (NTU) }\end{array}$ & $\begin{array}{c}\text { TRANS- } \\
\text { PAR- } \\
\text { ENCY } \\
\text { (SECCHI } \\
\text { DISK) } \\
\text { (IN) }\end{array}$ & $\begin{array}{l}\text { OXYGEN } \\
\text { DEMAND, } \\
\text { BIO- } \\
\text { CHEM- } \\
\text { ICAL, } \\
5 \text { DAY } \\
\text { (MG/L) }\end{array}$ & $\begin{array}{l}\text { COLI- } \\
\text { FORM, } \\
\text { FECAL, } \\
0.7 \\
\text { UM-MF } \\
\text { (COLS, } \\
100 \mathrm{ML} \text { ) }\end{array}$ & $\begin{array}{l}\text { CAICIUM } \\
\text { DIS- } \\
\text { SOLVED } \\
\text { (MG/I } \\
\text { AS CA) }\end{array}$ & $\begin{array}{l}\text { MAGNE- } \\
\text { SIUM, } \\
\text { DIS- } \\
\text { SOIVED } \\
\text { (MG/L } \\
\text { AS MG) }\end{array}$ & $\begin{array}{c}\text { ALKA- } \\
\text { IINITY } \\
\text { LAB } \\
\text { (MG/I } \\
\text { AS } \\
\text { CACO3) }\end{array}$ \\
\hline
\end{tabular}

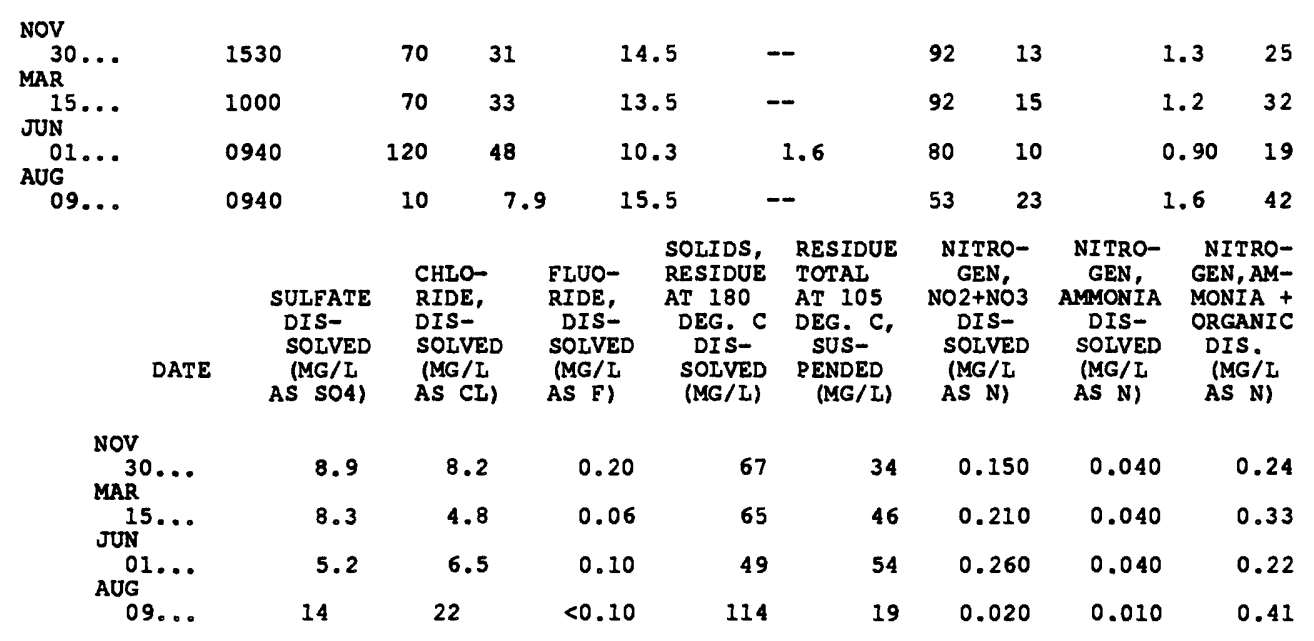


SURFACE-WATER SITES--Cont1 nued

02441498 TOMBIGBEE RIVER COLUMBUS BEND SR $11 B$ AT COLUMBUS, MS--Cont Inued

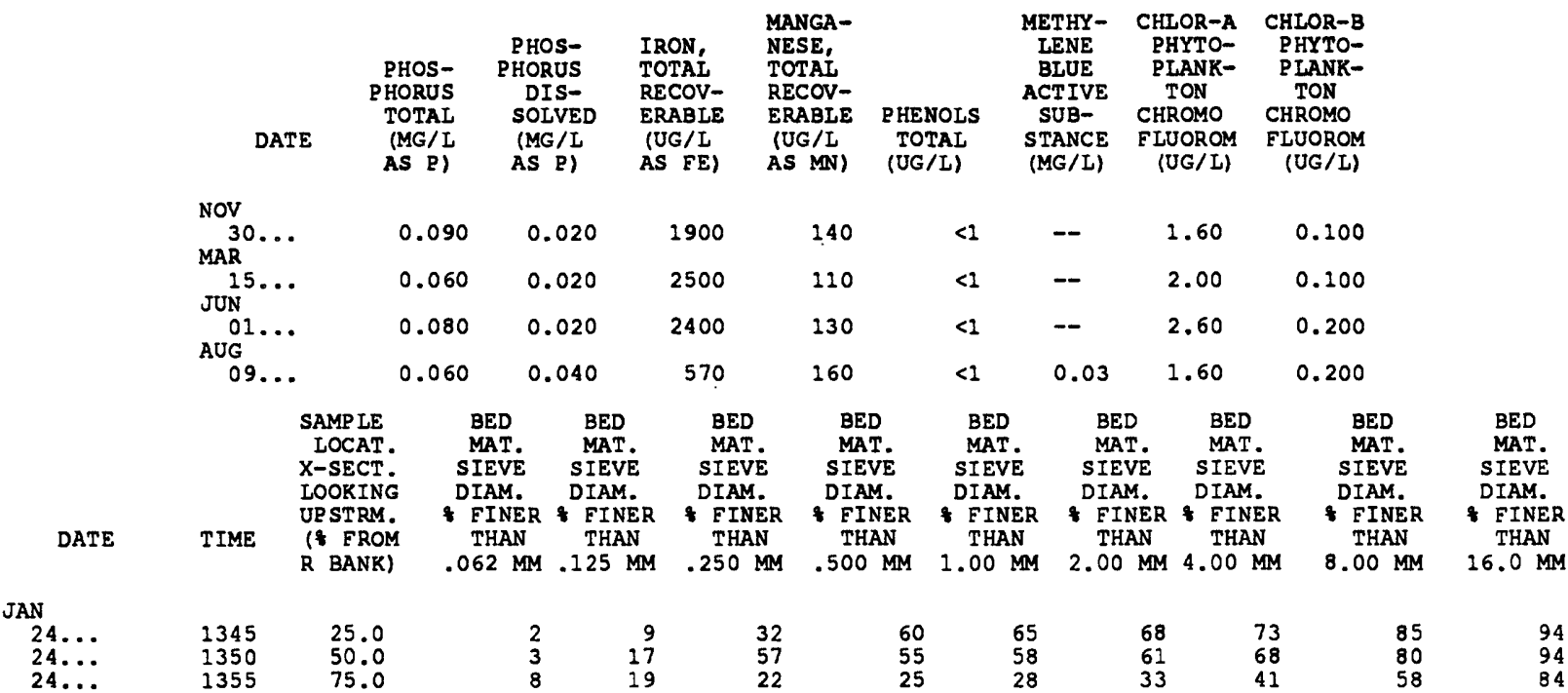

332751088261000 TTW ALICEVILLE LAKE COLUMBUS CUT

\begin{tabular}{|c|c|c|c|c|c|c|c|c|c|c|c|}
\hline DATE & TIME & $\begin{array}{l}\text { SAMPLE } \\
\text { LOCAT. } \\
\text { X-SECT. } \\
\text { LOOKING } \\
\text { UP STRM. } \\
\text { (\$ FROM } \\
\text { R BANK) }\end{array}$ & $\begin{array}{c}\text { BED } \\
\text { MAT. } \\
\text { SIEVE } \\
\text { DIAM. } \\
\text { EINER } \\
\text { THAN } \\
.062 \mathrm{MM}\end{array}$ & $\begin{array}{l}\text { BED } \\
\text { MAT. } \\
\text { SIEVE } \\
\text { DIAM. } \\
\text { FINER } \\
\text { THAN } \\
.125 \mathrm{MM}\end{array}$ & $\begin{array}{c}\text { BED } \\
\text { MAT. } \\
\text { SIEVE } \\
\text { DIAM. } \\
\text { FINER } \\
\text { THAN } \\
.250 \mathrm{MM}\end{array}$ & $\begin{array}{c}\text { BED } \\
\text { MAT. } \\
\text { SIEVE } \\
\text { DIAM. } \\
\text { FINER } \\
\text { THAN } \\
.500 \mathrm{MM}\end{array}$ & $\begin{array}{c}\text { BED } \\
\text { MAT. } \\
\text { SIEVE } \\
\text { DIAM. } \\
\text { FINER } \\
\text { THAN } \\
1.00 \mathrm{MM}\end{array}$ & $\begin{array}{c}\text { BED } \\
\text { MAT. } \\
\text { SIEVE } \\
\text { DIAM. } \\
\text { FINER } \\
\text { THAN } \\
2.00 \mathrm{MM}\end{array}$ & $\begin{array}{c}\text { BED } \\
\text { MAT. } \\
\text { SIEVE } \\
\text { DIAM. } \\
\text { FINER } \\
\text { THAN } \\
4.00 \mathrm{MM}\end{array}$ & $\begin{array}{c}\text { BED } \\
\text { MAT. } \\
\text { SIEVE } \\
\text { DIAM. } \\
\text { FINER } \\
\text { THAN } \\
8.00 \mathrm{MM}\end{array}$ & $\begin{array}{c}\text { BED } \\
\text { MAT. } \\
\text { SIEVE } \\
\text { DIAM. } \\
\text { FINER } \\
\text { THAN } \\
16.0 \mathrm{MMM}\end{array}$ \\
\hline $\begin{array}{l}W \\
24 \ldots \\
24 \ldots \\
24 \ldots\end{array}$ & $\begin{array}{l}1315 \\
1320 \\
1325\end{array}$ & $\begin{array}{l}25.0 \\
50.0 \\
75.0\end{array}$ & $\begin{array}{l}0 \\
0 \\
2\end{array}$ & $\begin{array}{l}0 \\
0 \\
5\end{array}$ & $\begin{array}{r}7 \\
16 \\
21\end{array}$ & $\begin{array}{l}87 \\
98 \\
70\end{array}$ & $\begin{array}{r}98 \\
100 \\
73\end{array}$ & $\begin{array}{r}98 \\
100 \\
75\end{array}$ & $\begin{array}{r}99 \\
100 \\
79\end{array}$ & $\begin{array}{r}100 \\
100 \\
86\end{array}$ & $\begin{array}{r}100 \\
100 \\
97\end{array}$ \\
\hline
\end{tabular}

02443500 LUXAPALLILA CREEK NEAR COLUMBUS, MS

\begin{tabular}{|c|c|c|c|c|c|c|c|c|c|c|c|}
\hline DATE & TIME & $\begin{array}{c}\text { DIS- } \\
\text { CHARGE, } \\
\text { INST. } \\
\text { CUBIC } \\
\text { FEET } \\
\text { PER } \\
\text { SECOND }\end{array}$ & $\begin{array}{l}\text { SPE- } \\
\text { CIFIC } \\
\text { CON- } \\
\text { DUCT- } \\
\text { ANCE } \\
\text { (US/CM) }\end{array}$ & $\begin{array}{c}\text { PH } \\
\text { (STAND- } \\
\text { ARD } \\
\text { UNITS) }\end{array}$ & $\begin{array}{c}\text { TEMPER- } \\
\text { ATURE } \\
\text { WATER } \\
\text { (DEG C) }\end{array}$ & $\begin{array}{l}\text { COLOR } \\
\text { (PLAT- } \\
\text { INUM- } \\
\text { COBALT } \\
\text { UNITS) }\end{array}$ & $\begin{array}{l}\text { TUR- } \\
\text { BID- } \\
\text { ITY } \\
\text { (NTU) }\end{array}$ & $\begin{array}{c}\text { TRANS- } \\
\text { PAR- } \\
\text { ENCY } \\
\text { (SECCHI } \\
\text { DISK) } \\
\text { (IN) }\end{array}$ & $\begin{array}{c}\text { OXYGEN, } \\
\text { DIS- } \\
\text { SOLVED } \\
\text { (MG/L) }\end{array}$ & $\begin{array}{c}\text { OXYGEN, } \\
\text { DIS- } \\
\text { SOLVED } \\
\text { (PER- } \\
\text { CENT } \\
\text { SATUR- } \\
\text { ATION) }\end{array}$ & $\begin{array}{c}\text { OXYGEN } \\
\text { DEMAND, } \\
\text { BIO- } \\
\text { CHEM- } \\
\text { ICAL, } \\
5 \text { DAY } \\
\text { (MG/L) }\end{array}$ \\
\hline
\end{tabular}

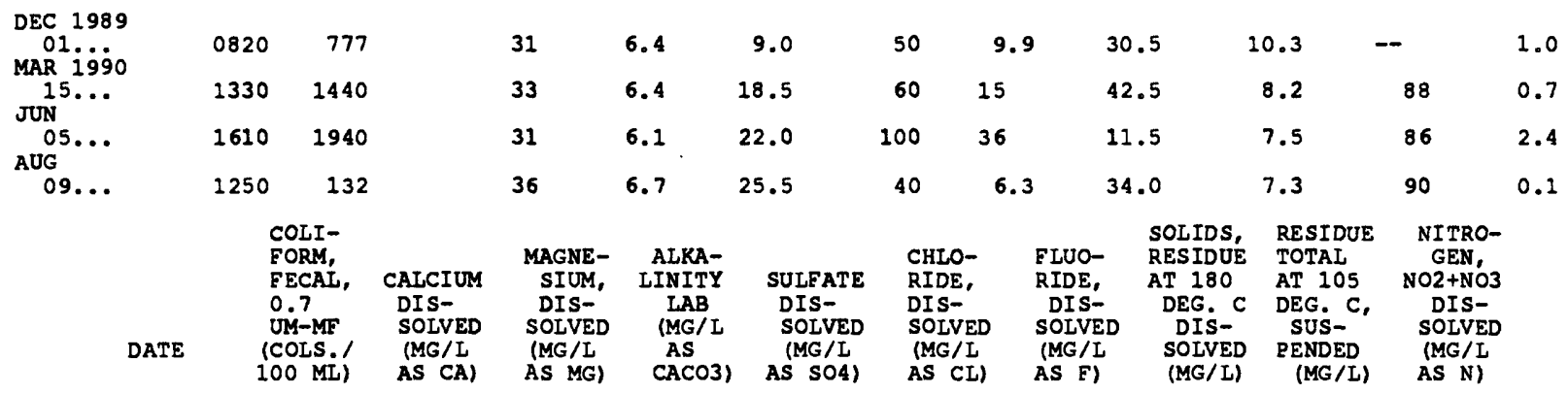

\begin{tabular}{|c|c|c|c|c|c|c|c|c|c|c|}
\hline $\begin{array}{c}\mathrm{DEC} \\
01 \ldots \\
M A R\end{array}$ & K240 & 1.9 & 0.80 & 3.3 & 3.4 & 2.9 & 0.10 & 22 & 8 & 0.070 \\
\hline JUN $^{15 \ldots}$ & 660 & 2.4 & 0.90 & 6.2 & 2.9 & 2.1 & $<0.05$ & 31 & 24 & 0.080 \\
\hline AUG $05 .$. & K960 & 2.0 & 0.90 & 5.1 & 2.3 & 1.9 & $<0.10$ & 31 & 55 & 0.170 \\
\hline $09 \ldots$ & 90 & 2.7 & 1.0 & 8.2 & 2.3 & 2.4 & $<0.10$ & 28 & 8 & 0.140 \\
\hline
\end{tabular}


02443500 LUXAPALIILA CREEK NEAR COLUMBUS, MS--Continued

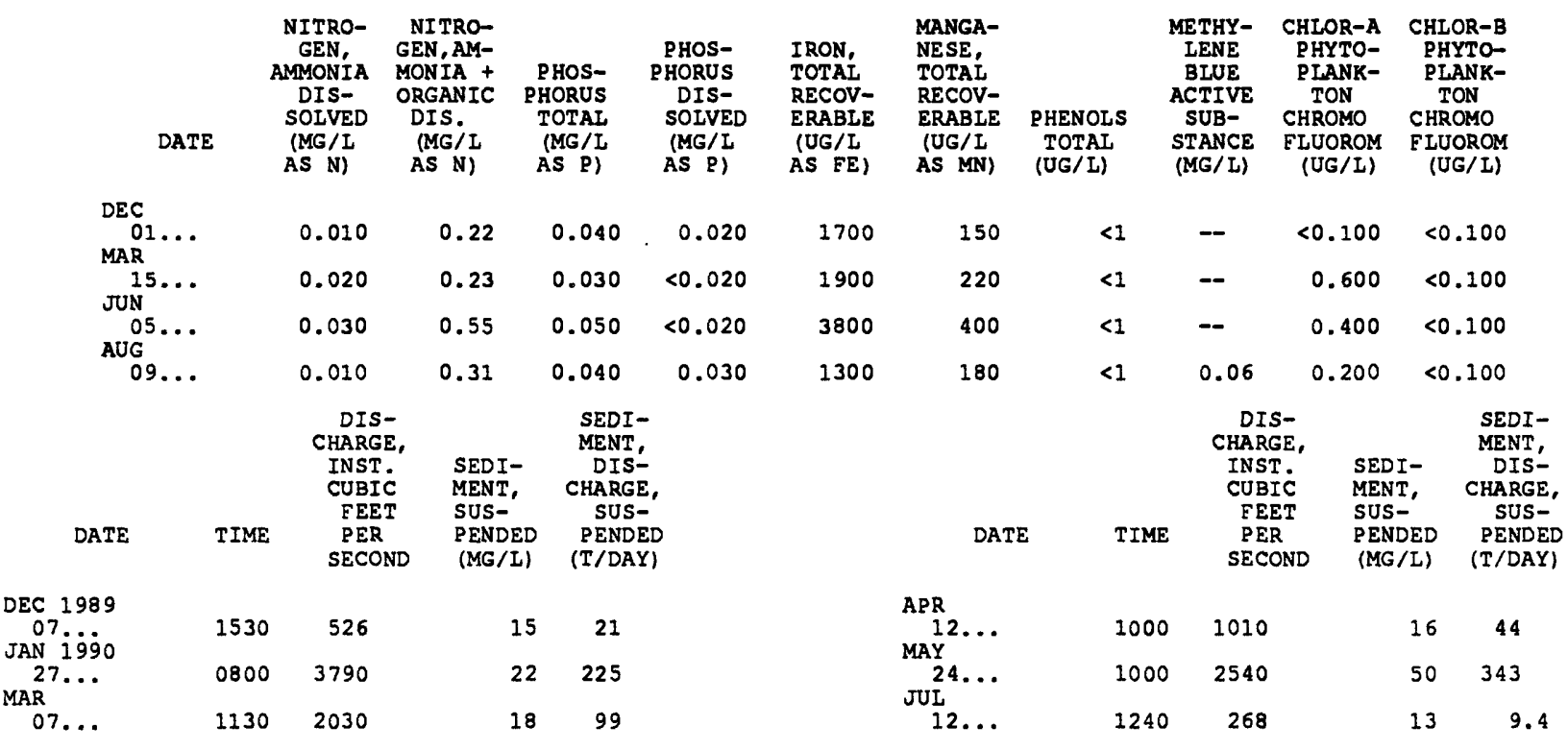

02443610 TOMBIGBEE RIVER PRATT CAMP SR 5HB BELOW COLUMBUS, MS

\begin{tabular}{|c|c|c|c|c|c|c|c|c|}
\hline DATE & TIME & $\begin{array}{l}\text { SAM- } \\
\text { PLING } \\
\text { DEPTH } \\
\text { (FEET) }\end{array}$ & $\begin{array}{l}\text { SPE- } \\
\text { CIEIC } \\
\text { CON- } \\
\text { DUCT- } \\
\text { ANCE } \\
\text { (US/CM) }\end{array}$ & $\begin{array}{c}\text { PH } \\
\text { (STAND- } \\
\text { ARD } \\
\text { UNITS) }\end{array}$ & $\begin{array}{c}\text { TEMPER- } \\
\text { ATURE } \\
\text { WATER } \\
\text { (DEG C) }\end{array}$ & $\begin{array}{c}\text { OXYGEN, } \\
\text { DIS- } \\
\text { SOLVED } \\
\text { (MG/L) }\end{array}$ & $\begin{array}{c}\text { OXYGEN, } \\
\text { DIS- } \\
\text { SOLVED } \\
\text { (PER- } \\
\text { CENT } \\
\text { SATUR- } \\
\text { ATION) }\end{array}$ & \\
\hline $\begin{array}{c}\text { DEC } \\
01 \ldots \\
01 \ldots \\
01 \ldots \\
01 \ldots \\
\text { MAR }\end{array}$ & $\begin{array}{l}1035 \\
1036 \\
1037 \\
1038\end{array}$ & $\begin{array}{l}5.00 \\
10.0 \\
13.0\end{array}$ & $\begin{array}{l}149 \\
152 \\
157 \\
154\end{array}$ & $\begin{array}{l}7.3 \\
7.2 \\
7.3 \\
7.2\end{array}$ & $\begin{array}{l}11.5 \\
11.0 \\
11.0 \\
11.0\end{array}$ & $\begin{array}{l}9.2 \\
8.9 \\
8.8 \\
8.5\end{array}$ & $\begin{array}{l}= \\
= \\
--\end{array}$ & \\
\hline $\begin{array}{c}16 \ldots \\
16 \ldots \\
16 \ldots \\
16 \ldots \\
\text { JUN }\end{array}$ & $\begin{array}{l}0931 \\
0932 \\
0933 \\
0934\end{array}$ & $\begin{array}{l}5.00 \\
10.0 \\
15.0\end{array}$ & $\begin{array}{l}140 \\
140 \\
140 \\
140\end{array}$ & $\begin{array}{l}7.4 \\
7.4 \\
7.4 \\
7.4\end{array}$ & $\begin{array}{l}16.0 \\
16.0 \\
16.5 \\
16.5\end{array}$ & $\begin{array}{l}9.4 \\
9.4 \\
9.3 \\
9.3\end{array}$ & $\begin{array}{l}96 \\
96 \\
95 \\
95\end{array}$ & \\
\hline $\begin{array}{c}05 \ldots \\
05 \ldots \\
05 \ldots \\
05 \ldots \\
\text { AUG }\end{array}$ & $\begin{array}{l}1255 \\
1256 \\
1257 \\
1258\end{array}$ & $\begin{array}{l}5.00 \\
10.0 \\
15.0\end{array}$ & $\begin{array}{l}138 \\
141 \\
142 \\
144\end{array}$ & $\begin{array}{l}7.4 \\
7.1 \\
7.1 \\
7.1\end{array}$ & $\begin{array}{l}27.5 \\
23.5 \\
23.5 \\
23.5\end{array}$ & $\begin{array}{l}8.6 \\
6.8 \\
6.7 \\
6.3\end{array}$ & $\begin{array}{l}110 \\
-- \\
--\end{array}$ & \\
\hline $\begin{array}{l}10 \ldots \\
10 \ldots \\
10 \ldots \\
10 \ldots\end{array}$ & $\begin{array}{l}0845 \\
0846 \\
0847 \\
0848\end{array}$ & $\begin{array}{l}5.00 \\
10.0 \\
15.0\end{array}$ & $\begin{array}{l}192 \\
191 \\
190 \\
188\end{array}$ & $\begin{array}{l}7.0 \\
7.1 \\
7.1 \\
6.7\end{array}$ & $\begin{array}{l}28.0 \\
28.0 \\
28.0 \\
27.5\end{array}$ & $\begin{array}{l}4.3 \\
4.3 \\
3.8 \\
1.4\end{array}$ & $\begin{array}{l}55 \\
=- \\
--\end{array}$ & \\
\hline TIME & $\begin{array}{l}\text { COLOR } \\
\text { (PLAT- } \\
\text { INUM- } \\
\text { COBALT } \\
\text { UNITS) }\end{array}$ & $\begin{array}{l}\text { TUR- } \\
\text { BID- } \\
\text { ITY } \\
\text { (NTU) }\end{array}$ & $\begin{array}{c}\text { TRANS- } \\
\text { PAR- } \\
\text { ENCY } \\
\text { (SECCHI } \\
\text { DI SK) } \\
\text { (IN) }\end{array}$ & $\begin{array}{l}\text { OXYGEN } \\
\text { DEMAND, } \\
\text { BIO- } \\
\text { CHEM- } \\
\text { ICAL, } \\
5 \text { DAY } \\
\text { (MG/L) }\end{array}$ & $\begin{array}{l}\text { COLI- } \\
\text { FORM, } \\
\text { FECAL, } \\
0.7 \\
\text { UM-ME } \\
\text { (COLS./ } \\
100 \mathrm{ML} \text { ) }\end{array}$ & $\begin{array}{l}\text { CALCIUM } \\
\text { DIS- } \\
\text { SOLVED } \\
\text { (MG/I } \\
\text { AS CA) }\end{array}$ & $\begin{array}{l}\text { MAGNE- } \\
\text { SIUM, } \\
\text { DIS- } \\
\text { SOIVED } \\
\text { (MG/L } \\
\text { AS MG) }\end{array}$ & $\begin{array}{c}\text { ALKA- } \\
\text { LINITY } \\
\text { LAB } \\
\text { (MG/I } \\
\text { AS } \\
\text { CACO3) }\end{array}$ \\
\hline
\end{tabular}

$\begin{array}{llll}\text { DEC } & & \\ \text { O1... } & 1035 & 50 & 26 \\ \text { MAR } & & & \\ \text { I6... } & 0930 & 40 & 72 \\ \underset{\text { OSN }}{\text { O5... }} & 1255 & 20 & 32 \\ \begin{array}{c}\text { AUG } \\ 10 \ldots\end{array} & 0845 & 10 & 16\end{array}$

$\begin{array}{llrlll}14.5 & -- & 230 & 22 & 1.3 & 45 \\ 10.5 & -- & >600 & 22 & 1.0 & 47 \\ 8.75 & 2.0 & 200 & 22 & 1.0 & 46 \\ 20.3 & -- & \text { K5 } & 22 & 1.5 & 47\end{array}$


SURFACE-WATER SITES--Cont1nued

02443610 TOMBIGBEE RIVER PRATT CAMP SR 5HB BELON COLUMBUS, MS--Continued

\begin{tabular}{|c|c|c|c|c|c|c|c|c|}
\hline DATE & $\begin{array}{l}\text { SULFATE } \\
\text { DIS- } \\
\text { SOLVED } \\
\text { (MG/L } \\
\text { AS SO4) }\end{array}$ & $\begin{array}{l}\text { CHLO- } \\
\text { RIDE, } \\
\text { DIS- } \\
\text { SOLVED } \\
\text { (MG/I } \\
\text { AS CI) }\end{array}$ & $\begin{array}{l}\text { FLUO- } \\
\text { RIDE, } \\
\text { DIS- } \\
\text { SOLVED } \\
\text { (MG/L } \\
\text { AS F) }\end{array}$ & $\begin{array}{c}\text { SOLIDS, } \\
\text { RESIDUE } \\
\text { AT } 180 \\
\text { DEG. C } \\
\text { DIS- } \\
\text { SOLVED } \\
(M G / L)\end{array}$ & $\begin{array}{l}\text { RESIDUE } \\
\text { TOTAL } \\
\text { AT 105 } \\
\text { DEG. C, } \\
\text { SUS- } \\
\text { PENDED } \\
\text { (MG/L) }\end{array}$ & $\begin{array}{c}\text { NITRQ- } \\
\text { GEN, } \\
\text { NO2+NO3 } \\
\text { DIS- } \\
\text { SOLVED } \\
\text { (MG/L } \\
\text { AS N) }\end{array}$ & $\begin{array}{c}\text { NITRO- } \\
\text { GEN, } \\
\text { AMMONIA } \\
\text { DIS- } \\
\text { SOLVED } \\
\text { (MG/I } \\
\text { AS N) }\end{array}$ & $\begin{array}{l}\text { NITRO- } \\
\text { GEN,AM- } \\
\text { MONIA + } \\
\text { ORGANIC } \\
\text { DIS. } \\
\text { (MG/L } \\
\text { AS N) }\end{array}$ \\
\hline $\begin{array}{l}\mathrm{DEC} \\
01 \ldots \\
\mathrm{MAR}\end{array}$ & 14 & 7.2 & 0.20 & 93 & 31 & 0.110 & 0.060 & 0.36 \\
\hline JUN $16 \ldots$ & 8.9 & 3.9 & 0.09 & 84 & 92 & 0.230 & 0.040 & 0.23 \\
\hline$\underset{A U G}{05 \ldots}$ & 9.2 & 4.8 & 0.20 & 92 & 52 & 0.710 & 0.040 & 0.49 \\
\hline $10 \ldots$ & 14 & 18 & $<0.10$ & 110 & 27 & $<0.020$ & 0.010 & 0.38 \\
\hline DATE & $\begin{array}{l}\text { PHOS- } \\
\text { PHORUS } \\
\text { TOTAL } \\
\text { (MG/L } \\
\text { AS P) }\end{array}$ & $\begin{array}{c}\text { PHOS- } \\
\text { PHORUS } \\
\text { DIS- } \\
\text { SOLVED } \\
\text { (MG/L } \\
\text { AS P) }\end{array}$ & $\begin{array}{l}\text { IRON, } \\
\text { TOTAL } \\
\text { RECOV- } \\
\text { ERABLE } \\
\text { (UG/I } \\
\text { AS FE) }\end{array}$ & $\begin{array}{l}\text { MANGA- } \\
\text { NESE, } \\
\text { TOTAL } \\
\text { RECOV- } \\
\text { ERABLE } \\
\text { (UG/L } \\
\text { AS MN) }\end{array}$ & $\begin{array}{l}\text { P HENOLS } \\
\text { TOTAL } \\
\text { (UG/L) }\end{array}$ & $\begin{array}{l}\text { METHY- } \\
\text { LENE } \\
\text { BLUE } \\
\text { ACTIVE } \\
\text { SUB- } \\
\text { STANCE } \\
\text { (MG/L) }\end{array}$ & $\begin{array}{c}\text { CHLOR-A } \\
\text { PHYTO- } \\
\text { PLANK- } \\
\text { TON } \\
\text { CHROMO } \\
\text { FIUOROM } \\
\text { (UG/L) }\end{array}$ & $\begin{array}{c}\text { CHLOR-B } \\
\text { P HYTO- } \\
\text { PLANK- } \\
\text { TON } \\
\text { CHROMO } \\
\text { FLUOROM } \\
\text { (UG/L) }\end{array}$ \\
\hline $\begin{array}{l}\text { DEC } \\
01 \ldots \\
\text { MAR }\end{array}$ & 0.100 & 0.040 & 1500 & 160 & $<1$ & -- & 1.70 & $<0.100$ \\
\hline $16 .$. & 0.110 & 0.020 & 4300 & 100 & $<1$ & -- & 2.20 & 0.200 \\
\hline $\begin{array}{l}05 \ldots \\
\text { AUG }\end{array}$ & 0.140 & 0.040 & 1600 & 80 & $<1$ & -- & 3.20 & 0.300 \\
\hline $10 \ldots$ & 0.080 & 0.030 & 920 & 270 & $<1$ & 0.07 & 2.70 & 0.500 \\
\hline
\end{tabular}

02444157 TOMBIGBEE RIVER AT ST HWY 86 NEAR PICKENSVILIE, AL

\begin{tabular}{|c|c|c|c|c|c|}
\hline DATE & TIME & $\begin{array}{l}\text { SAMPLE } \\
\text { IOCAT. } \\
\text { X-SECT. } \\
\text { LOOKING } \\
\text { UPSTRM. } \\
\text { (4 FROM } \\
\text { R BANK) }\end{array}$ & $\begin{array}{c}\text { DIS- } \\
\text { CHARGE, } \\
\text { INST. } \\
\text { CUBIC } \\
\text { FEET } \\
\text { PER } \\
\text { SECOND }\end{array}$ & $\begin{array}{l}\text { SEDI- } \\
\text { MENT, } \\
\text { SUS- } \\
\text { PENDED } \\
\text { (MG/L) }\end{array}$ & $\begin{array}{r}\text { SEI } \\
\mathrm{MEI} \\
\mathrm{D} \\
\mathrm{CHA} \\
\mathrm{S} \\
\mathrm{PE} \\
\langle\mathrm{T} / \mathrm{I}\end{array}$ \\
\hline \multicolumn{6}{|l|}{ OCT } \\
\hline $\begin{array}{l}11 \ldots \\
11 \ldots \\
11 \ldots\end{array}$ & $\begin{array}{l}0903 \\
0910 \\
0915\end{array}$ & $\begin{array}{l}25.0 \\
50.0 \\
75.0\end{array}$ & $\begin{array}{l}570 \\
570 \\
570\end{array}$ & $\begin{array}{r}11 \\
7 \\
8\end{array}$ & $\begin{array}{l}17 \\
11\end{array}$ \\
\hline \multicolumn{6}{|l|}{ Nov } \\
\hline $\begin{array}{r}20 \ldots \\
20 \ldots \\
20 \ldots \\
\text { JAN }\end{array}$ & $\begin{array}{l}0950 \\
0955 \\
1000\end{array}$ & $\begin{array}{l}25.0 \\
50.0 \\
75.0\end{array}$ & $\begin{array}{l}9960 \\
9960 \\
9960\end{array}$ & $\begin{array}{l}24 \\
27 \\
28\end{array}$ & $\begin{array}{l}645 \\
726 \\
753\end{array}$ \\
\hline $\begin{array}{l}03 \ldots \\
03 \ldots \\
03 \ldots\end{array}$ & $\begin{array}{l}1000 \\
1010 \\
1015\end{array}$ & $\begin{array}{l}25.0 \\
50.0 \\
75.0\end{array}$ & $\begin{array}{l}19300 \\
19300 \\
19300\end{array}$ & $\begin{array}{r}100 \\
96 \\
108\end{array}$ & $\begin{array}{l}5210 \\
5000 \\
5630\end{array}$ \\
\hline \multicolumn{6}{|l|}{ FEB } \\
\hline $\begin{array}{r}23 \ldots \\
23 \ldots \\
23 \ldots \\
\text { APR }\end{array}$ & $\begin{array}{l}1215 \\
1220 \\
1225\end{array}$ & $\begin{array}{l}25.0 \\
50.0 \\
75.0\end{array}$ & $\begin{array}{l}37600 \\
37600 \\
37600\end{array}$ & $\begin{array}{l}117 \\
112 \\
118\end{array}$ & $\begin{array}{l}11900 \\
11400 \\
12000\end{array}$ \\
\hline $\begin{array}{r}04 \ldots \\
04 \ldots \\
04 \ldots \\
\text { MAY }\end{array}$ & $\begin{array}{l}1050 \\
1055 \\
1100\end{array}$ & $\begin{array}{l}25.0 \\
50.0 \\
75.0\end{array}$ & $\begin{array}{l}4120 \\
4120 \\
4120\end{array}$ & $\begin{array}{l}18 \\
12 \\
11\end{array}$ & $\begin{array}{l}200 \\
133 \\
122\end{array}$ \\
\hline $\begin{array}{l}14 \ldots \\
14 \ldots \\
14 \ldots\end{array}$ & $\begin{array}{l}1035 \\
1040 \\
1045\end{array}$ & $\begin{array}{l}25.0 \\
50.0 \\
75.0\end{array}$ & $\begin{array}{l}4070 \\
4070 \\
4070\end{array}$ & $\begin{array}{l}20 \\
14 \\
11\end{array}$ & $\begin{array}{l}220 \\
154 \\
121\end{array}$ \\
\hline \multicolumn{6}{|l|}{ JUN } \\
\hline $\begin{array}{r}25 \ldots \\
25 \ldots \\
25 \ldots \\
\text { AUG }\end{array}$ & $\begin{array}{l}1030 \\
1035 \\
1040\end{array}$ & $\begin{array}{l}25.0 \\
50.0 \\
75.0\end{array}$ & $\begin{array}{l}4980 \\
4980 \\
4980\end{array}$ & $\begin{array}{l}27 \\
30 \\
36\end{array}$ & $\begin{array}{l}36 \\
40 \\
48\end{array}$ \\
\hline $06 \ldots$ & 0940 & $\begin{array}{l}25.0 \\
50.0\end{array}$ & $\begin{array}{l}1300 \\
1300\end{array}$ & $\begin{array}{l}21 \\
22\end{array}$ & \\
\hline \multicolumn{6}{|l|}{ SEP } \\
\hline $\begin{array}{l}17 \ldots \\
17 \ldots \\
17 \ldots\end{array}$ & $\begin{array}{l}0930 \\
0935 \\
0940\end{array}$ & $\begin{array}{l}25.0 \\
50.0 \\
75.0\end{array}$ & $\begin{array}{l}366 \\
366 \\
366\end{array}$ & $\begin{array}{l}12 \\
12 \\
13\end{array}$ & \\
\hline
\end{tabular}


SURFACE-WATER SITES--Cont1nued

02444210 TOMBIGBEE RIVER BIG CREEK BEND NEAR PICKENSVIIIE, AL

\begin{tabular}{|c|c|c|c|c|c|c|c|}
\hline DATE & TIME & $\begin{array}{l}\text { SAM- } \\
\text { PIING } \\
\text { DEPTH } \\
\text { (FEET) }\end{array}$ & $\begin{array}{l}\text { SPE- } \\
\text { CIFIC } \\
\text { CON- } \\
\text { DUCT- } \\
\text { ANCE } \\
\text { (US/CM) }\end{array}$ & $\begin{array}{c}\text { PH } \\
\text { (STAND- } \\
\text { ARD } \\
\text { UNITS) }\end{array}$ & $\begin{array}{c}\text { TEMPER- } \\
\text { ATURE } \\
\text { WATER } \\
\text { (DEG C) }\end{array}$ & $\begin{array}{c}\text { OXYGEN, } \\
\text { DIS- } \\
\text { SOLVED } \\
\text { (MG/I) }\end{array}$ & $\begin{array}{c}\text { OXYGEN, } \\
\text { DIS- } \\
\text { SOLVED } \\
\text { (PER- } \\
\text { CENT } \\
\text { SATUR- } \\
\text { ATION) }\end{array}$ \\
\hline
\end{tabular}

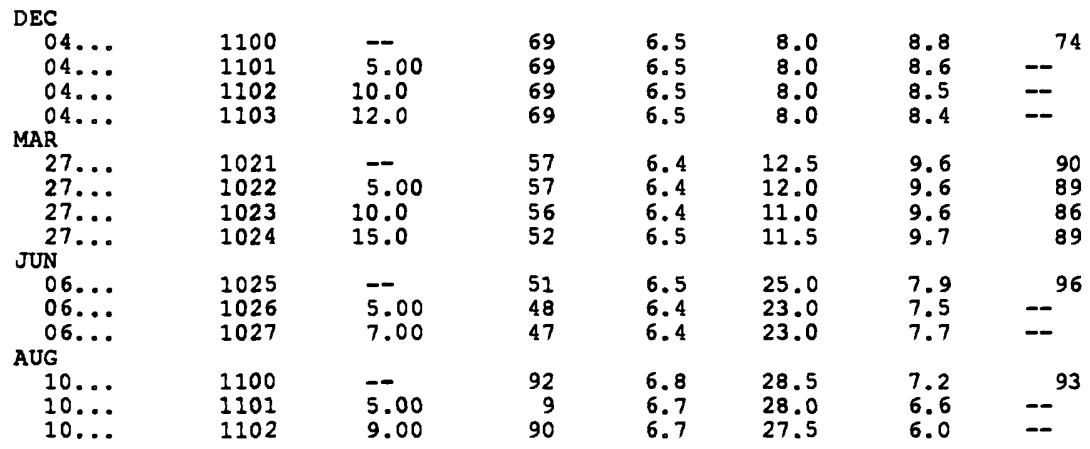

\begin{tabular}{|c|c|c|c|c|c|c|c|c|c|}
\hline DATE & TIME & $\begin{array}{l}\text { COLOR } \\
\text { (PLAT- } \\
\text { INUM- } \\
\text { COBALT } \\
\text { UNITS) }\end{array}$ & $\begin{array}{l}\text { TUR- } \\
\text { BID- } \\
\text { ITY } \\
\text { (NTU) }\end{array}$ & $\begin{array}{c}\text { TRANS- } \\
\text { PAR- } \\
\text { ENCY } \\
\text { (SECCHI } \\
\text { DISK) } \\
\text { (IN) }\end{array}$ & $\begin{array}{l}\text { OXYGEN } \\
\text { DEMAND, } \\
\text { BIO- } \\
\text { CHEM- } \\
\text { ICAL, } \\
5 \text { DAY } \\
\text { (MG/L) }\end{array}$ & $\begin{array}{l}\text { COLI- } \\
\text { FORM, } \\
\text { FECAL, } \\
0.7 \\
\text { UM-MF } \\
\text { (COLS.', } \\
\text { 100 ML) }\end{array}$ & $\begin{array}{l}\text { CALCIUM } \\
\text { DIS- } \\
\text { SOLVED } \\
\text { (MG/L } \\
\text { AS CA) }\end{array}$ & $\begin{array}{l}\text { MAGNE- } \\
\text { SIUM, } \\
\text { DIS- } \\
\text { SOLVED } \\
\text { (MG/L } \\
\text { AS MG) }\end{array}$ & $\begin{array}{c}\text { ALKA- } \\
\text { LINITY } \\
\text { LAB } \\
\text { (MG/L } \\
\text { AS } \\
\text { CACO3) }\end{array}$ \\
\hline
\end{tabular}

\begin{tabular}{|c|c|c|}
\hline $\begin{array}{l}\mathrm{DEC} \\
04 \ldots\end{array}$ & 1100 & 10 \\
\hline $27 \ldots$ & 1020 & 20 \\
\hline $\begin{array}{l}06 \ldots \\
\text { AUG }\end{array}$ & 1025 & 20 \\
\hline & 1100 & $<5$ \\
\hline
\end{tabular}

$\begin{array}{llllll}4.50 & -- & -- & 5.8 & 1.1 & 9.4 \\ 19.5 & -- & 280 & 4.6 & 1.1 & 11 \\ 15.5 & 1.2 & 230 & 4.9 & 1.1 & 12 \\ 16.5 & -- & 26 & 10 & 2.1 & 28\end{array}$

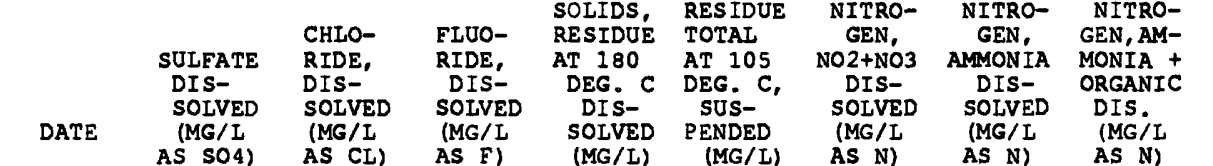

\begin{tabular}{|c|c|c|c|c|c|c|c|c|}
\hline $\begin{array}{c}\mathrm{DEC} \\
\mathrm{O}_{4} \ldots\end{array}$ & 7.1 & 3.0 & 0.10 & 41 & 200 & 0.080 & 0.480 & 0.72 \\
\hline $27 \ldots$ & 5.8 & 2.2 & $<0.10$ & 34 & 66 & 0.110 & 0.030 & $<0.20$ \\
\hline $\begin{array}{l}06 \ldots \\
A \cup G\end{array}$ & 4.8 & 2.3 & 0.10 & 43 & 25 & 0.080 & $<0.010$ & 0.22 \\
\hline $10 \ldots$ & 7.6 & 4.7 & $<0.10$ & 54 & 21 & $<0.020$ & 0.010 & 0.23 \\
\hline DATE & $\begin{array}{l}\text { PHOS- } \\
\text { PHORUS } \\
\text { TOTAI } \\
\text { (MG/I } \\
\text { AS P) }\end{array}$ & $\begin{array}{l}\text { PHOS- } \\
\text { PHORUS } \\
\text { DIS- } \\
\text { SOIVED } \\
\text { (MG/L } \\
\text { AS P) }\end{array}$ & $\begin{array}{l}\text { IRON, } \\
\text { TOTAL } \\
\text { RECOV- } \\
\text { ERABLE } \\
\text { (UG/L } \\
\text { AS FE) }\end{array}$ & $\begin{array}{l}\text { MANGA- } \\
\text { NESE, } \\
\text { TOTAL } \\
\text { RECOV- } \\
\text { ERABLE } \\
\text { (UG/I } \\
\text { AS MN) }\end{array}$ & $\begin{array}{l}\text { PHENOLS } \\
\text { TOTAL } \\
\text { (UG/L) }\end{array}$ & $\begin{array}{l}\text { METHY- } \\
\text { IENE } \\
\text { BLUE } \\
\text { ACTIVE } \\
\text { SUB- } \\
\text { STANCE } \\
\text { (MG/L) }\end{array}$ & $\begin{array}{c}\text { CHLOR-A } \\
\text { PHYTO- } \\
\text { PIANK- } \\
\text { TON } \\
\text { CHROMO } \\
\text { FLUOROM } \\
\text { (UG/L) }\end{array}$ & $\begin{array}{c}\text { CHLOR-B } \\
\text { PHYTO- } \\
\text { PIANK- } \\
\text { TON } \\
\text { CHROMO } \\
\text { FLUOROM } \\
\text { (UG/I) }\end{array}$ \\
\hline $\begin{array}{l}\text { DEC } \\
04 \ldots \\
\text { MAR }\end{array}$ & 0.240 & 0.020 & 9000 & 730 & -- & -- & 1.20 & 0.100 \\
\hline $27 \ldots$ & 0.060 & 0.040 & 2900 & 450 & $<1$ & -- & 2.00 & $<0.100$ \\
\hline$\underset{A \cup G}{06 \ldots}$ & 0.080 & $<0.020$ & 1400 & 270 & $<1$ & -- & 8.00 & 0.900 \\
\hline $10 \ldots$ & 0.070 & 0.040 & 1400 & 540 & $<1$ & 0.03 & 2.80 & 0.700 \\
\hline
\end{tabular}


SURFACE-WATER SITES--Cont1nued

02444500 TOMBIGBEE RIVER NEAR COCHRANE, AL

\begin{tabular}{|c|c|c|c|c|}
\hline DATE & TIME & $\begin{array}{l}\text { SAMP LE } \\
\text { LOCAT. } \\
\text { X-SECT. } \\
\text { LOOKING } \\
\text { UP STRM. } \\
\text { (* FROM } \\
\text { R BANK) }\end{array}$ & $\begin{array}{c}\text { DIS- } \\
\text { CHARGE, } \\
\text { INST. } \\
\text { CUBIC } \\
\text { FEET } \\
\text { PER } \\
\text { SECOND }\end{array}$ & $\begin{array}{l}\text { SEDI- } \\
\text { MENT, } \\
\text { SUS- } \\
\text { PENDED } \\
\text { (MG/L) }\end{array}$ \\
\hline $\begin{array}{l}T \\
11 \ldots \\
11 \ldots \\
11 \ldots\end{array}$ & $\begin{array}{l}1030 \\
1035 \\
1040\end{array}$ & $\begin{array}{l}25.0 \\
50.0 \\
75.0\end{array}$ & $\begin{array}{l}E 603 \\
E 603 \\
E 603\end{array}$ & $\begin{array}{l}27 \\
13 \\
14\end{array}$ \\
\hline $\begin{array}{l}20 \ldots \\
20 \ldots \\
20 \ldots \\
N\end{array}$ & $\begin{array}{l}1130 \\
1140 \\
1145\end{array}$ & $\begin{array}{l}25.0 \\
50.0 \\
75.0\end{array}$ & $\begin{array}{l}\text { E7860 } \\
\text { E7860 } \\
\text { E7860 }\end{array}$ & $\begin{array}{l}30 \\
30 \\
30\end{array}$ \\
\hline $\begin{array}{l}03 \ldots \\
03 \ldots \\
03 \ldots \\
B\end{array}$ & $\begin{array}{l}1130 \\
1135 \\
1140\end{array}$ & $\begin{array}{l}25.0 \\
50.0 \\
75.0\end{array}$ & $\begin{array}{l}\text { E17700 } \\
\text { E17700 } \\
\text { E17700 }\end{array}$ & $\begin{array}{l}112 \\
111 \\
107\end{array}$ \\
\hline $\begin{array}{l}23 \ldots \\
23 \ldots \\
23 \ldots \\
R\end{array}$ & $\begin{array}{l}1045 \\
1050 \\
1055\end{array}$ & $\begin{array}{l}25.0 \\
50.0 \\
75.0\end{array}$ & $\begin{array}{l}\text { E38600 } \\
\text { E38600 } \\
\text { E38600 }\end{array}$ & $\begin{array}{r}89 \\
91 \\
102\end{array}$ \\
\hline $\begin{array}{l}04 \ldots \\
04 \ldots \\
04 \ldots \\
Y\end{array}$ & $\begin{array}{l}1225 \\
1230 \\
1235\end{array}$ & $\begin{array}{l}25.0 \\
50.0 \\
75.0\end{array}$ & $\begin{array}{l}E 7800 \\
E 7800 \\
E 7800\end{array}$ & $\begin{array}{l}10 \\
14 \\
12\end{array}$ \\
\hline $\begin{array}{l}14 \ldots \\
14 \ldots \\
14 \ldots \\
N\end{array}$ & $\begin{array}{l}1145 \\
1150 \\
1155\end{array}$ & $\begin{array}{l}25.0 \\
50.0 \\
75.0\end{array}$ & $\begin{array}{l}E 7780 \\
E 7780 \\
E 7780\end{array}$ & $\begin{array}{l}17 \\
14 \\
15\end{array}$ \\
\hline $\begin{array}{l}25 \ldots \\
25 \ldots \\
25 \ldots\end{array}$ & $\begin{array}{l}1140 \\
1145 \\
1150\end{array}$ & $\begin{array}{l}25.0 \\
50.0 \\
75.0\end{array}$ & $\begin{array}{l}\text { E4170 } \\
\text { E4170 } \\
\text { E4170 }\end{array}$ & $\begin{array}{l}27 \\
42 \\
43\end{array}$ \\
\hline $\begin{array}{l}06 \ldots \\
06 \ldots \\
06 \ldots \\
0\end{array}$ & $\begin{array}{l}1045 \\
1050 \\
1055\end{array}$ & $\begin{array}{l}25.0 \\
50.0 \\
75.0\end{array}$ & $\begin{array}{l}E 2340 \\
E 2340 \\
E 2340\end{array}$ & $\begin{array}{l}24 \\
24 \\
68\end{array}$ \\
\hline $\begin{array}{l}17 \ldots \\
17 \ldots \\
17 \ldots\end{array}$ & $\begin{array}{l}1055 \\
1100 \\
1105\end{array}$ & $\begin{array}{l}25.0 \\
50.0 \\
75.0\end{array}$ & $\begin{array}{l}E 1300 \\
E 1300 \\
E 1300\end{array}$ & $\begin{array}{l}14 \\
15 \\
15\end{array}$ \\
\hline
\end{tabular}

02446500 SIPSEY RIVER NEAR ELROD, AL

\begin{tabular}{|c|c|c|c|c|c|c|c|c|c|}
\hline & DATE & TIME & $\begin{array}{l}\text { SAM- } \\
\text { PLING } \\
\text { DEPTH } \\
\text { (FEET) }\end{array}$ & $\begin{array}{l}\text { SPE- } \\
\text { CIFIC } \\
\text { CON- } \\
\text { DUCT- } \\
\text { ANCE } \\
\text { (US/CM) }\end{array}$ & $\begin{array}{c}\text { PH } \\
\text { (STAND- } \\
\text { ARD } \\
\text { UNITS) }\end{array}$ & $\begin{array}{c}\text { TEMPER- } \\
\text { ATURE } \\
\text { WATER } \\
\text { (DEG C) }\end{array}$ & $\begin{array}{c}\text { OXYGEN, } \\
\text { DIS- } \\
\text { SOLVED } \\
\text { (MG/L) }\end{array}$ & $\begin{array}{c}\text { OXYGEN, } \\
\text { DIS- } \\
\text { SOLVED } \\
\text { (PER- } \\
\text { CENT } \\
\text { SATUR- } \\
\text { ATION) }\end{array}$ & \\
\hline & $\begin{array}{r}\text { NOV } \\
27 \ldots \\
27 \ldots \\
27 \ldots \\
\text { MAR }\end{array}$ & $\begin{array}{l}0900 \\
0901 \\
0902\end{array}$ & $\begin{array}{l}-- \\
5.00 \\
7.00\end{array}$ & $\begin{array}{l}42 \\
43 \\
43\end{array}$ & $\begin{array}{l}6.0 \\
6.0 \\
6.0\end{array}$ & $\begin{array}{l}11.5 \\
11.5 \\
11.5\end{array}$ & $\begin{array}{l}7.7 \\
7.7 \\
7.7\end{array}$ & $-{ }^{72}$ & \\
\hline & $\begin{array}{r}12 \ldots \\
12 \ldots \\
12 \ldots \\
M A Y\end{array}$ & $\begin{array}{l}0941 \\
0942 \\
0943\end{array}$ & $\begin{array}{l}\overline{5.00} \\
10.0\end{array}$ & $\begin{array}{l}67 \\
69 \\
69\end{array}$ & $\begin{array}{l}6.4 \\
6.4 \\
6.4\end{array}$ & $\begin{array}{l}18.0 \\
18.0 \\
17.5\end{array}$ & $\begin{array}{l}7.3 \\
7.2 \\
7.2\end{array}$ & $\begin{array}{l}77 \\
76 \\
76\end{array}$ & \\
\hline & $\begin{array}{l}29 \ldots \\
29 . \ldots \\
\text { AUG }\end{array}$ & $\begin{array}{l}1000 \\
1001\end{array}$ & 4.00 & $\begin{array}{l}63 \\
64\end{array}$ & $\begin{array}{l}6.4 \\
6.4\end{array}$ & $\begin{array}{l}20.5 \\
20.5\end{array}$ & $\begin{array}{l}6.6 \\
6.5\end{array}$ & $--^{74}$ & \\
\hline & $06 \ldots$ & $\begin{array}{l}0930 \\
0931\end{array}$ & 2.00 & $\begin{array}{l}93 \\
93\end{array}$ & $\begin{array}{l}6.7 \\
6.7\end{array}$ & $\begin{array}{l}26.0 \\
26.0\end{array}$ & $\begin{array}{l}6.8 \\
6.8\end{array}$ & $--^{84}$ & \\
\hline DATE & TIME & $\begin{array}{l}\text { DIS- } \\
\text { CHARGE, } \\
\text { INST. } \\
\text { CUBIC } \\
\text { FEET } \\
\text { PER } \\
\text { SECOND }\end{array}$ & $\begin{array}{l}\text { COLOR } \\
\text { (PLAT- } \\
\text { INUM- } \\
\text { COBALT } \\
\text { UNITS) }\end{array}$ & $\begin{array}{l}\text { TUR- } \\
\text { BID- } \\
\text { ITY } \\
\text { (NTU) }\end{array}$ & $\begin{array}{l}\text { TRANS- } \\
\text { PAR- } \\
\text { ENCY } \\
\text { (SECCHI } \\
\text { DISK) } \\
\text { (IN) }\end{array}$ & $\begin{array}{l}\text { OXYGEN } \\
\text { DEMAND, } \\
\text { BIO- } \\
\text { CHEM- } \\
\text { ICAL, } \\
5 \text { DAY } \\
\text { (MG/L) }\end{array}$ & $\begin{array}{l}\text { COLI- } \\
\text { FORM, } \\
\text { FECAL, } \\
0.7 \\
\text { UM-MF } \\
\text { (COLS.' } \\
100 \mathrm{ML} \text { ) }\end{array}$ & $\begin{array}{l}\text { CALCIUM } \\
\text { DIS- } \\
\text { SOLVED } \\
\text { (MG/L } \\
\text { AS CA) }\end{array}$ & $\begin{array}{l}\text { MAGNE- } \\
\text { SIUM, } \\
\text { DIS- } \\
\text { SOLVED } \\
\text { (MG/L } \\
\text { AS MG) }\end{array}$ \\
\hline \multicolumn{10}{|l|}{ NOV } \\
\hline $\operatorname{MAR}^{27 \ldots}$ & 0900 & 2490 & 80 & 16 & 23.0 & -- & 480 & 2.4 & 1.5 \\
\hline${ }_{M A Y}^{12} \cdots$ & 0940 & 1620 & 30 & 5.9 & 43.5 & - & 140 & 4.7 & 3.0 \\
\hline${ }_{A \cup G}^{29} \ldots$ & 1000 & 1190 & 50 & 6.1 & 30.5 & 0.6 & 180 & 4.6 & 3.1 \\
\hline $06 \ldots$ & 0930 & 140 & 20 & 3.4 & 24.5 & - & 42 & - & - \\
\hline
\end{tabular}


SURFACE-WATER SITES--Cont Inued

02446500 SIRSEY RIVER NEAR ELROD, AI--Cont Inued

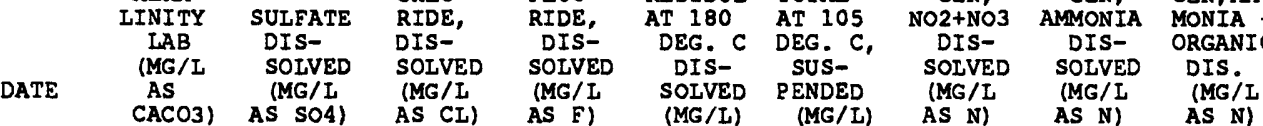

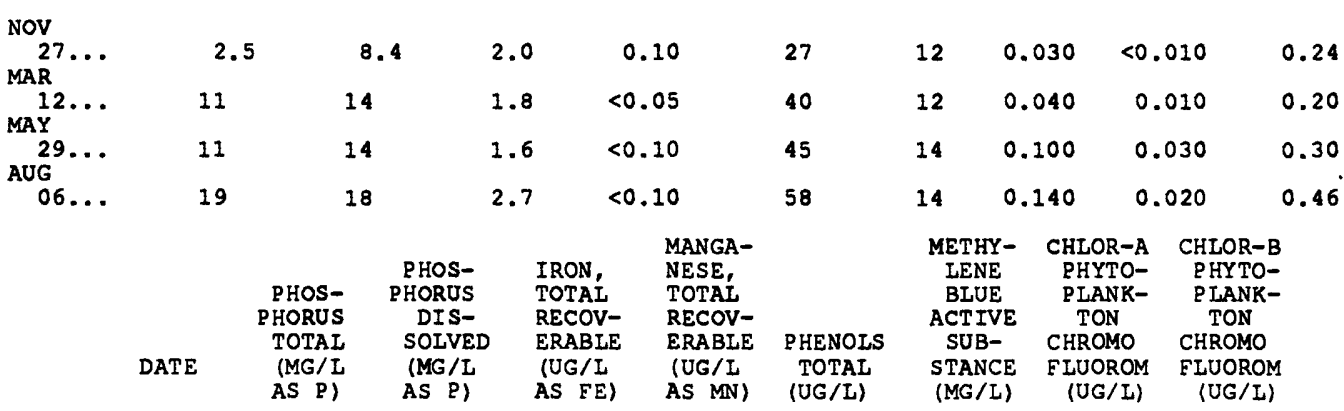

\begin{tabular}{|c|c|c|c|c|c|c|c|c|}
\hline $\begin{array}{l}\text { NOV } \\
27 \ldots \\
\text { MAR }\end{array}$ & 0.060 & 0.020 & 1700 & 120 & $<1$ & -- & 0.400 & 0.100 \\
\hline$\frac{12}{M A Y}$ & 0.020 & $<0.020$ & 1000 & 90 & $<1$ & - & 0.700 & $<0.100$ \\
\hline AUG $29 .$. & 0.020 & $<0.020$ & 1400 & 200 & $<1$ & -- & 0.300 & $<0.100$ \\
\hline $06 \ldots$ & 0.050 & 0.040 & 1100 & 300 & $<1$ & 0.06 & 2.00 & 0.200 \\
\hline
\end{tabular}

02447010 TOMBIGBEE RIVER COOKS BEND NEAR WARSAW, AL

\begin{tabular}{|c|c|c|c|c|c|c|c|}
\hline DATE & TIME & $\begin{array}{l}\text { SAM- } \\
\text { PIING } \\
\text { DEPTH } \\
\text { (EEET) }\end{array}$ & $\begin{array}{l}\text { SPE- } \\
\text { CIFIC } \\
\text { CON- } \\
\text { DUCT- } \\
\text { ANCE } \\
\text { (US/CM) }\end{array}$ & $\begin{array}{c}\text { PH } \\
\text { (STAND- } \\
\text { ARD } \\
\text { UNITS) }\end{array}$ & $\begin{array}{c}\text { TEMPER- } \\
\text { ATURE } \\
\text { WATER } \\
\text { (DEG C) }\end{array}$ & $\begin{array}{c}\text { OXYGEN, } \\
\text { DIS- } \\
\text { SOLVED } \\
\text { (MG/I) }\end{array}$ & $\begin{array}{c}\text { OXYGEN, } \\
\text { DIS- } \\
\text { SOLVED } \\
\text { (PER- } \\
\text { CENT } \\
\text { SATUR- } \\
\text { ATION) }\end{array}$ \\
\hline \multicolumn{8}{|l|}{ DEC } \\
\hline $\begin{array}{l}05 \ldots \\
05 \ldots \\
05 \ldots \\
05 \ldots \\
05 \ldots \\
05 \ldots \\
05 \ldots \\
05 \ldots \\
05 \ldots \\
\text { MAR }\end{array}$ & $\begin{array}{l}0915 \\
0916 \\
0917 \\
0918 \\
0919 \\
0920 \\
0921 \\
0922 \\
0923\end{array}$ & $\begin{array}{c}-- \\
5.00 \\
10.0 \\
15.0 \\
20.0 \\
25.0 \\
30.0 \\
35.0 \\
38.0\end{array}$ & $\begin{array}{l}90 \\
90 \\
91 \\
91 \\
89 \\
89 \\
89 \\
89 \\
89\end{array}$ & $\begin{array}{l}7.1 \\
7.1 \\
7.1 \\
7.1 \\
7.1 \\
7.1 \\
7.1 \\
7.1 \\
7.1\end{array}$ & $\begin{array}{l}9.5 \\
9.5 \\
9.5 \\
9.5 \\
9.5 \\
9.5 \\
9.5 \\
9.5 \\
9.5\end{array}$ & $\begin{array}{l}10.9 \\
10.8 \\
10.8 \\
10.8 \\
10.7 \\
10.7 \\
10.7 \\
10.6 \\
10.6\end{array}$ & $\begin{array}{l}96 \\
95 \\
95 \\
95 \\
94 \\
94 \\
94 \\
93 \\
93\end{array}$ \\
\hline $\begin{array}{l}27 \ldots \\
27 \ldots \\
27 \ldots \\
27 \ldots \\
27 \ldots \\
27 \ldots \\
27 \ldots \\
\text { JUN }\end{array}$ & $\begin{array}{l}1326 \\
1327 \\
1328 \\
1329 \\
1330 \\
1331 \\
1332\end{array}$ & $\begin{array}{l}5.00 \\
10.0 \\
15.0 \\
20.0 \\
25.0 \\
27.0\end{array}$ & $\begin{array}{l}65 \\
65 \\
65 \\
65 \\
65 \\
65 \\
65\end{array}$ & $\begin{array}{l}6.8 \\
6.8 \\
6.8 \\
6.8 \\
6.8 \\
6.8 \\
6.8\end{array}$ & $\begin{array}{l}16.0 \\
15.5 \\
15.5 \\
15.5 \\
15.5 \\
15.5 \\
15.5\end{array}$ & $\begin{array}{l}10.5 \\
10.5 \\
10.4 \\
10.4 \\
10.3 \\
10.3 \\
10.3\end{array}$ & $\begin{array}{l}106 \\
-- \\
-- \\
-- \\
--\end{array}$ \\
\hline $\begin{array}{l}06 \ldots \\
06 \ldots \\
06 \ldots \\
06 \ldots \\
06 \ldots \\
06 \ldots \\
06 \ldots \\
06 \ldots \\
06 \ldots \\
06 \ldots \\
06 \ldots \\
\text { AUG }\end{array}$ & $\begin{array}{l}1410 \\
1411 \\
1412 \\
1413 \\
1414 \\
1415 \\
1416 \\
1417 \\
1418 \\
1419 \\
1420\end{array}$ & $\begin{array}{c}-- \\
5.00 \\
10.0 \\
15.0 \\
20.0 \\
25.0 \\
30.0 \\
35.0 \\
40.0 \\
45.0 \\
48.0\end{array}$ & $\begin{array}{l}109 \\
110 \\
109 \\
110 \\
109 \\
109 \\
109 \\
109 \\
109 \\
109 \\
109\end{array}$ & $\begin{array}{l}7.0 \\
7.0 \\
7.0 \\
7.0 \\
7.0 \\
7.0 \\
7.0 \\
7.0 \\
7.0 \\
7.0 \\
7.0\end{array}$ & $\begin{array}{l}25.5 \\
25.0 \\
25.0 \\
25.0 \\
25.0 \\
25.0 \\
25.0 \\
25.0 \\
25.0 \\
25.0 \\
25.0\end{array}$ & $\begin{array}{l}8.2 \\
8.4 \\
8.4 \\
8.4 \\
8.4 \\
8.4 \\
8.4 \\
8.3 \\
8.2 \\
8.2 \\
8.2\end{array}$ & $\begin{array}{l}100 \\
= \\
= \\
= \\
= \\
= \\
= \\
= \\
=\end{array}$ \\
\hline $\begin{array}{l}13 \ldots \\
13 \ldots \\
13 \ldots \\
13 \ldots \\
13 \ldots \\
13 \ldots \\
13 \ldots\end{array}$ & $\begin{array}{l}1215 \\
1216 \\
1217 \\
1218 \\
1219 \\
1220 \\
1221\end{array}$ & $\begin{array}{c}-- \\
5.00 \\
10.0 \\
15.0 \\
20.0 \\
25.0 \\
30.0\end{array}$ & $\begin{array}{l}159 \\
162 \\
162 \\
162 \\
162 \\
162 \\
163\end{array}$ & $\begin{array}{l}7.2 \\
6.9 \\
6.9 \\
6.9 \\
6.8 \\
6.8 \\
6.7\end{array}$ & $\begin{array}{l}30.0 \\
29.5 \\
29.5 \\
29.5 \\
29.0 \\
29.0 \\
29.0\end{array}$ & $\begin{array}{l}6.9 \\
5.2 \\
4.8 \\
4.8 \\
4.6 \\
4.3 \\
2.5\end{array}$ & $\begin{array}{l}92 \\
-- \\
-- \\
-- \\
--\end{array}$ \\
\hline
\end{tabular}


02447010 TOMBIGBEE RIVER COOKS BEND NEAR WARSAW, AL--Cont Inued

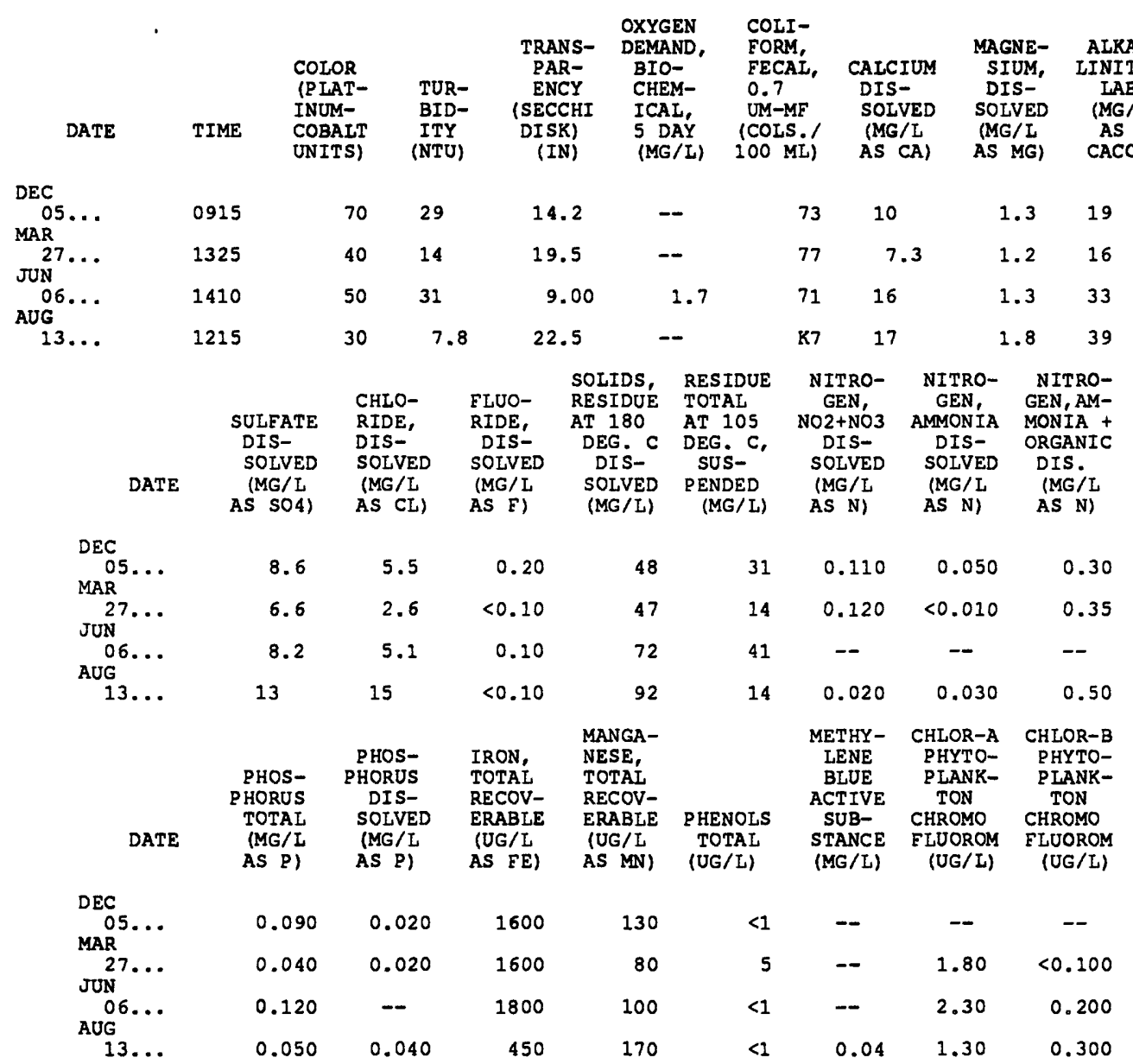

02448000 NOXUBEE RIVER AT MACON, MS

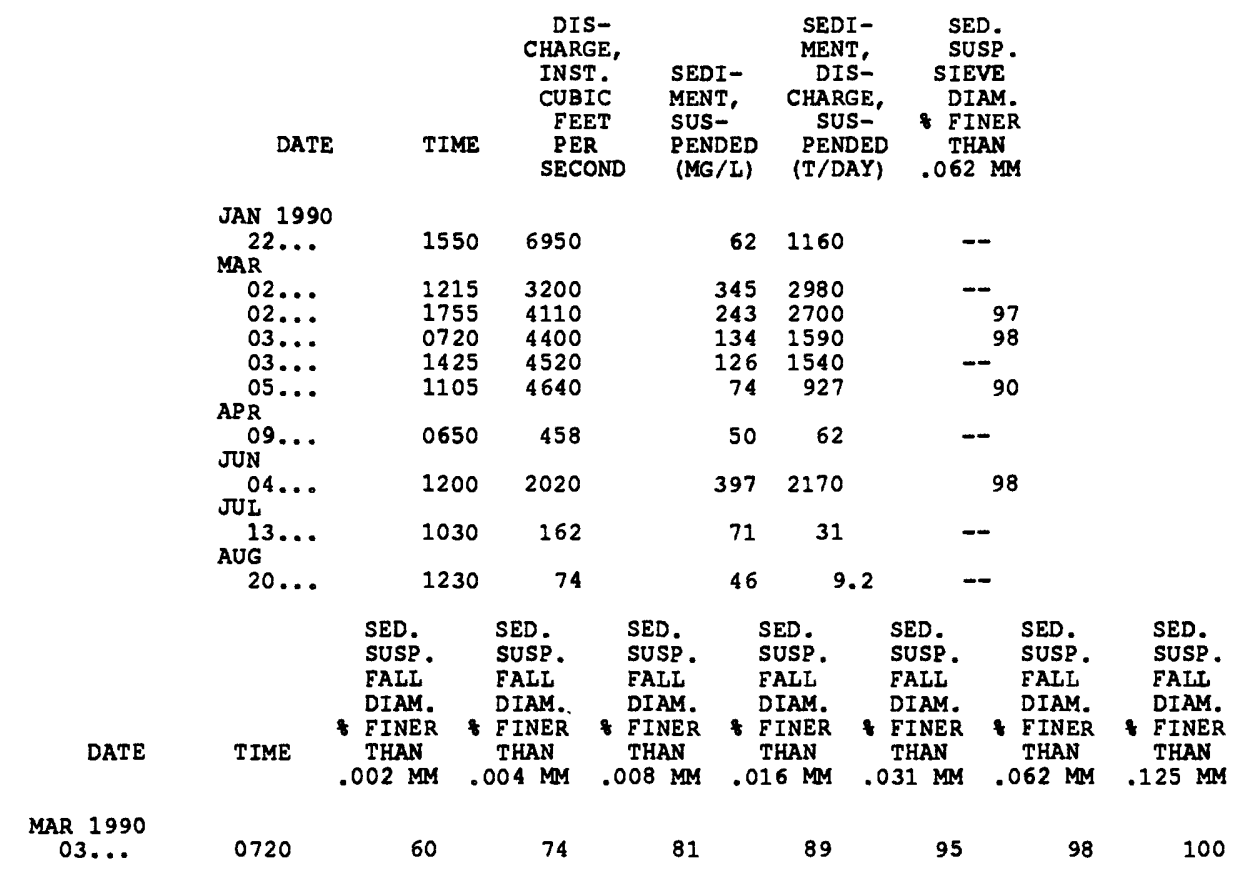


SURFACE-WATER SITES--Cont I nued

02448000 NOXUBEE RIVER AT MACON, MS--Cont1nued

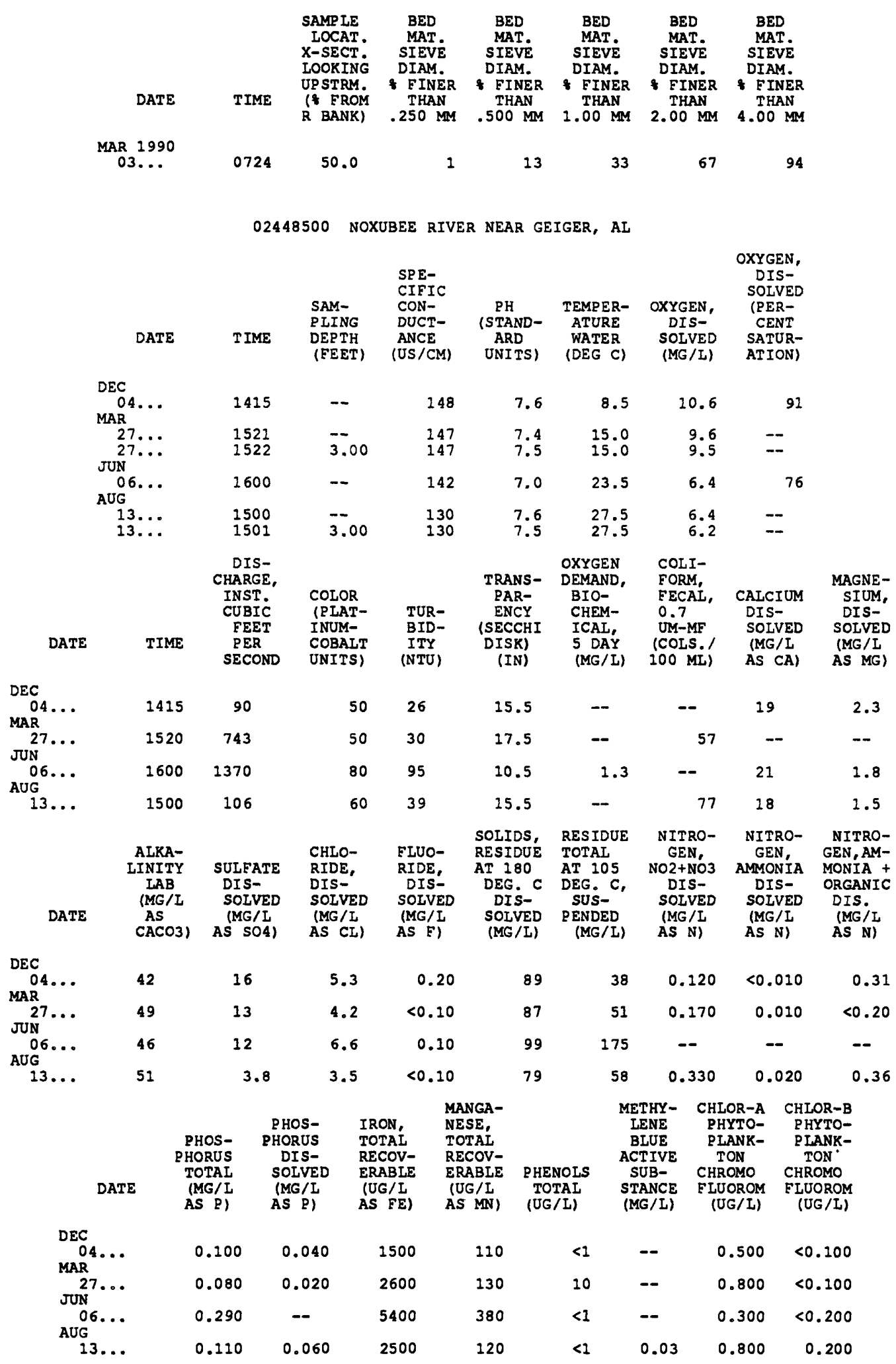


SURFACE-WATER SITES--Cont 1 nued

02449000 TOMBIGBEE RIVER AT GAINESVILLE, AL

\begin{tabular}{|c|c|c|c|c|c|c|c|c|c|c|c|}
\hline DATE & TIME & $\begin{array}{c}\text { DIS- } \\
\text { CHARGE, } \\
\text { INST. } \\
\text { CUBIC } \\
\text { FEET } \\
\text { PER } \\
\text { SECOND }\end{array}$ & $\begin{array}{l}\text { SPE- } \\
\text { CIFIC } \\
\text { CON- } \\
\text { DUCT- } \\
\text { ANCE } \\
\text { (US/CM) }\end{array}$ & $\begin{array}{c}\text { PH } \\
\text { (STAND- } \\
\text { ARD } \\
\text { UNITS) }\end{array}$ & $\begin{array}{c}\text { TEMPER- } \\
\text { ATURE } \\
\text { WATER } \\
\text { (DEG C) }\end{array}$ & $\begin{array}{l}\text { TUR- } \\
\text { BID- } \\
\text { ITY } \\
\text { (NTU) }\end{array}$ & $\begin{array}{c}\text { OXYGEN, } \\
\text { DIS- } \\
\text { SOLVED } \\
(M G / L)\end{array}$ & $\begin{array}{c}\text { OXYGEN, } \\
\text { DIS- } \\
\text { SOLVED } \\
\text { (PER- } \\
\text { CENT } \\
\text { SATUR- } \\
\text { ATION) }\end{array}$ & $\begin{array}{l}\text { COLI- } \\
\text { FORM, } \\
\text { FECAL, } \\
0.7 \\
\text { UM-MF } \\
\text { (COLS./ } \\
100 \mathrm{ML} \text { ) }\end{array}$ & $\begin{array}{l}\text { STREP - } \\
\text { TOCOCCI } \\
\text { FECAL, } \\
\text { KF AGAR } \\
\text { (COLS. } \\
\text { PER } \\
100 \mathrm{ML} \text { ) }\end{array}$ & $\begin{array}{l}\text { HARD- } \\
\text { NESS } \\
\text { TOTAL } \\
\text { (MG/I } \\
\text { AS } \\
\text { CACO3) }\end{array}$ \\
\hline $\begin{array}{l}1 \\
11 \\
v\end{array}$ & 0930 & 6300 & 98 & 7.3 & 21.5 & 21 & 8.6 & 97 & 42 & 85 & 38 \\
\hline $07 \ldots$ & 1000 & 17300 & 114 & 7.6 & 17.0 & 33 & 9.9 & 103 & $>600$ & $>1000$ & 46 \\
\hline $05 \ldots$ & 1215 & 6000 & 86 & 7.2 & 10.0 & 20 & 11.8 & 105 & 85 & 59 & 30 \\
\hline $10 \ldots$ & 1100 & 58000 & 95 & 6.8 & 9.0 & 28 & 11.2 & 96 & 310 & 410 & 37 \\
\hline $20 \ldots$ & 1400 & 128000 & 64 & 7.0 & 13.0 & 37 & 8.8 & 83 & $>600$ & 250 & 27 \\
\hline $28 \ldots$ & 1000 & 12800 & 79 & 7.0 & 15.5 & 15 & 10.9 & 108 & 27 & K15 & 28 \\
\hline $\begin{array}{l}02 \ldots \\
24 \ldots \\
N\end{array}$ & $\begin{array}{l}1000 \\
1025\end{array}$ & $\begin{array}{r}7690 \\
25500\end{array}$ & $\begin{array}{l}131 \\
--\end{array}$ & -2.6 & 25.5 & 28.3 & --1 & -- & $--^{K 9}$ & $--\quad 27$ & $\begin{array}{l}39 \\
46\end{array}$ \\
\hline L 7 . . & 0955 & 11800 & 115 & 7.1 & 25.0 & 26 & 8.4 & 102 & K97 & 58 & 45 \\
\hline $18 \ldots$ & 1000 & 3500 & 122 & -- & 29.0 & 11 & -- & -- & 34 & 30 & 38 \\
\hline $14 \ldots$ & 0900 & 220 & 149 & 7.3 & 29.0 & 7.4 & 7.3 & -- & K3 & K6 & 49 \\
\hline $11 \ldots$ & 1100 & 385 & 173 & 7.2 & 30.0 & 3.0 & 6.7 & 89 & K7 & K2 & 55 \\
\hline DATE & $\begin{array}{l}\text { CALCIUM } \\
\text { DIS- } \\
\text { SOLVED } \\
\text { (MG/I } \\
\text { AS CA) }\end{array}$ & $\begin{array}{l}\text { MAGNE- } \\
\text { SIUM, } \\
\text { DIS- } \\
\text { SOIVED } \\
\text { (MG/L } \\
\text { AS MG) }\end{array}$ & $\begin{array}{l}\text { SODIUM, } \\
\text { DIS- } \\
\text { SOIVED } \\
\text { (MG/L } \\
\text { AS NA) }\end{array}$ & $\begin{array}{l}\text { SODIUM } \\
\text { PERCENT }\end{array}$ & $\begin{array}{c}\text { SODIUM } \\
\text { AD- } \\
\text { SORP- } \\
\text { TION } \\
\text { RATIO }\end{array}$ & $\begin{array}{l}\text { POTAS- } \\
\text { SIUM, } \\
\text { DIS- } \\
\text { SOIVED } \\
\text { (MG/I } \\
\text { AS K) }\end{array}$ & $\begin{array}{l}\text { BICAR- } \\
\text { BONATE } \\
\text { WATER } \\
\text { DIS IT } \\
\text { FIELD } \\
\text { MG/I AS } \\
\text { HCO3 }\end{array}$ & $\begin{array}{l}\text { ALKA- } \\
\text { IINITY } \\
\text { WAT DIS } \\
\text { TOT IT } \\
\text { FIELD } \\
\text { MG/L AS } \\
\text { CACO3 }\end{array}$ & $\begin{array}{l}\text { SULFATE } \\
\text { DIS- } \\
\text { SOLVED } \\
\text { (MG/I } \\
\text { AS SO4) }\end{array}$ & $\begin{array}{l}\text { CHLO- } \\
\text { RIDE, } \\
\text { DIS- } \\
\text { SOLVED } \\
\text { (MG/I } \\
\text { AS CL) }\end{array}$ & $\begin{array}{l}\text { FLUO- } \\
\text { RIDE, } \\
\text { DIS- } \\
\text { SOIVED } \\
\text { (MG/I } \\
\text { AS F) }\end{array}$ \\
\hline
\end{tabular}

\begin{tabular}{|c|c|c|c|c|c|c|c|c|c|c|c|}
\hline $\begin{array}{l}\text { OCT } \\
11 \ldots \\
\text { NOV }\end{array}$ & 13 & 1.4 & 3.0 & 14 & 0.2 & 2.0 & 22 & 22 & 11 & 4.6 & 0.10 \\
\hline DEC & 16 & 1.5 & 4.3 & 16 & 0.3 & 2.0 & 44 & 36 & 11 & 7.6 & 0.10 \\
\hline $\begin{array}{c}05 \ldots \\
\text { JAN }\end{array}$ & 9.5 & 1.5 & 2.9 & 16 & 0.2 & 2.1 & 24 & 19 & 10 & 4.9 & $<0.10$ \\
\hline FEB $10 \ldots$ & 13 & 0.98 & 2.5 & 12 & 0.2 & 2.0 & 33 & 27 & 9.0 & 3.9 & 0.10 \\
\hline${ }_{\text {MAR }}^{20 \ldots}$ & 9.2 & 0.87 & 1.7 & 12 & 0.1 & 1.4 & 18 & 14 & 6.0 & 2.4 & 0.10 \\
\hline $\begin{array}{l}28 \ldots \\
\text { MAY }\end{array}$ & 9.2 & 1.3 & 2.2 & 14 & 0.2 & 1.1 & 18 & 15 & 8.3 & 3.3 & 0.10 \\
\hline $\begin{array}{l}02 \ldots \\
24 \ldots \\
\text { JUN }\end{array}$ & $\begin{array}{l}13 \\
16\end{array}$ & $\begin{array}{l}1.6 \\
1.5\end{array}$ & $\begin{array}{l}4.9 \\
4.4\end{array}$ & $\begin{array}{l}21 \\
17\end{array}$ & $\begin{array}{l}0.3 \\
0.3\end{array}$ & $\begin{array}{l}1.3 \\
1.5\end{array}$ & $--^{29}$ & $--^{24}$ & $\begin{array}{l}11 \\
8.9\end{array}$ & $\begin{array}{l}9.3 \\
8.3\end{array}$ & $\begin{array}{r}0.10 \\
<0.10\end{array}$ \\
\hline${ }_{\text {JUL }}^{07 \ldots}$ & 16 & 1.3 & 3.5 & 14 & 0.2 & 1.7 & 31 & 25 & 8.4 & 5.9 & $<0.10$ \\
\hline AUG & 13 & 1.4 & 5.7 & 24 & 0.4 & 1.6 & 35 & 29 & 8.3 & 8.4 & $<0.10$ \\
\hline $\operatorname{SEP}^{14 \ldots}$ & 17 & 1.6 & 8.5 & 26 & 0.5 & 1.8 & 33 & 27 & 10 & 15 & $<0.10$ \\
\hline $11 \ldots$ & 19 & 1.9 & 12 & 31 & 0.7 & 2.0 & 50 & 41 & 14 & 19 & $<0.10$ \\
\hline
\end{tabular}


02449000 TOMBIGBEE RIVER AT GAINESVILLE, AL--Cont1nued

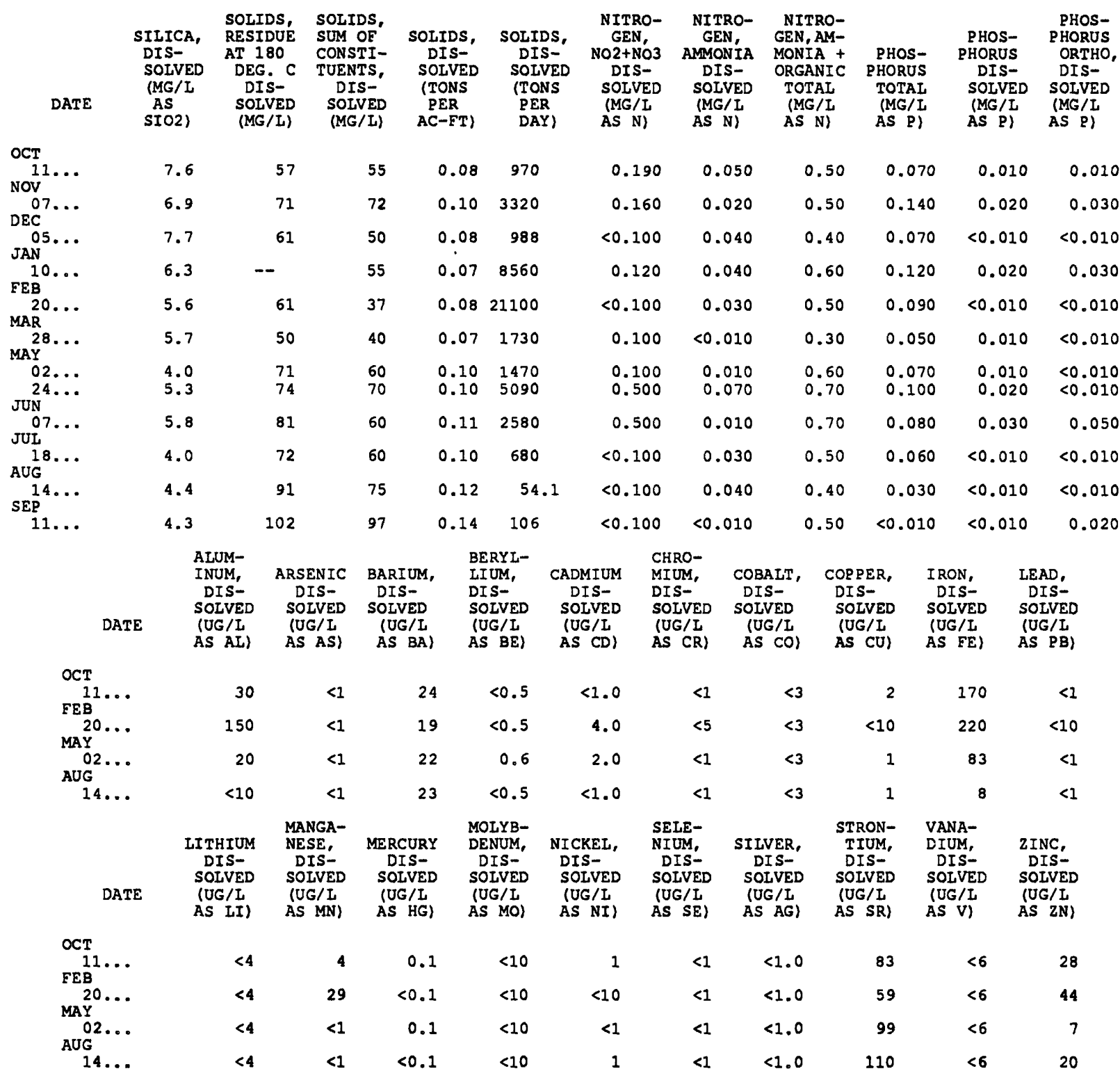


SURFACE-WATER SITES--Continued

02449000 TOMBIGBEE RIVER AT GAINESVILIE, AL--Cont1 nued

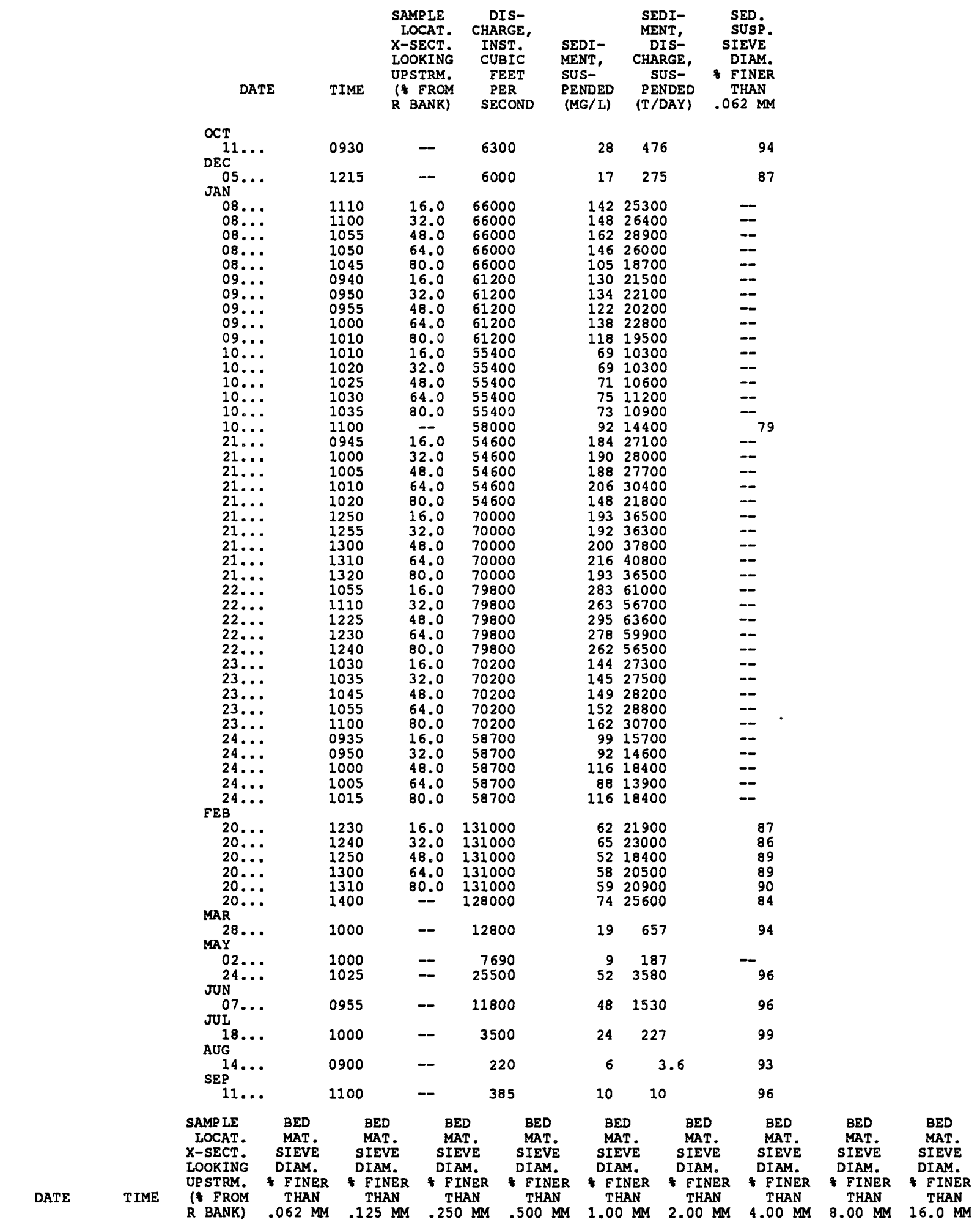

\begin{tabular}{|c|c|c|c|c|c|c|c|c|c|c|c|}
\hline JAN & & & & & & & & & & & \\
\hline $\begin{array}{l}23 . \ldots \\
23 . \ldots \\
23 . . .\end{array}$ & $\begin{array}{l}1115 \\
1125 \\
1135\end{array}$ & $\begin{array}{l}25.0 \\
50.0 \\
75.0\end{array}$ & $\begin{array}{l}0 \\
0 \\
0\end{array}$ & $\begin{array}{l}0 \\
0 \\
1\end{array}$ & $\begin{array}{r}1 \\
1 \\
17\end{array}$ & $\begin{array}{l}11 \\
19 \\
99\end{array}$ & $\begin{array}{r}13 \\
35 \\
100\end{array}$ & $\begin{array}{r}15 \\
39 \\
100\end{array}$ & $\begin{array}{r}18 \\
46 \\
100\end{array}$ & $\begin{array}{r}30 \\
65 \\
100\end{array}$ & $\begin{array}{r}72 \\
96 \\
100\end{array}$ \\
\hline $\begin{array}{l}\text { AUG } \\
14 \ldots \\
14 \ldots \\
14 \ldots\end{array}$ & $\begin{array}{l}0910 \\
0920 \\
0930\end{array}$ & $\begin{array}{l}25.0 \\
50.0 \\
75.0\end{array}$ & $\begin{array}{r}2 \\
0 \\
<1\end{array}$ & $\begin{array}{l}7 \\
0 \\
1\end{array}$ & $\begin{array}{r}20 \\
5 \\
11\end{array}$ & $\begin{array}{l}32 \\
12 \\
91\end{array}$ & $\begin{array}{l}34 \\
18 \\
97\end{array}$ & $\begin{array}{l}37 \\
24 \\
98\end{array}$ & $\begin{array}{l}43 \\
33 \\
98\end{array}$ & $\begin{array}{l}60 \\
61 \\
99\end{array}$ & $\begin{array}{r}96 \\
93 \\
100\end{array}$ \\
\hline
\end{tabular}


02467001 TOMBIGBEE RIVER BELOW DEMOPOLIS LOCK AND DAM, AL

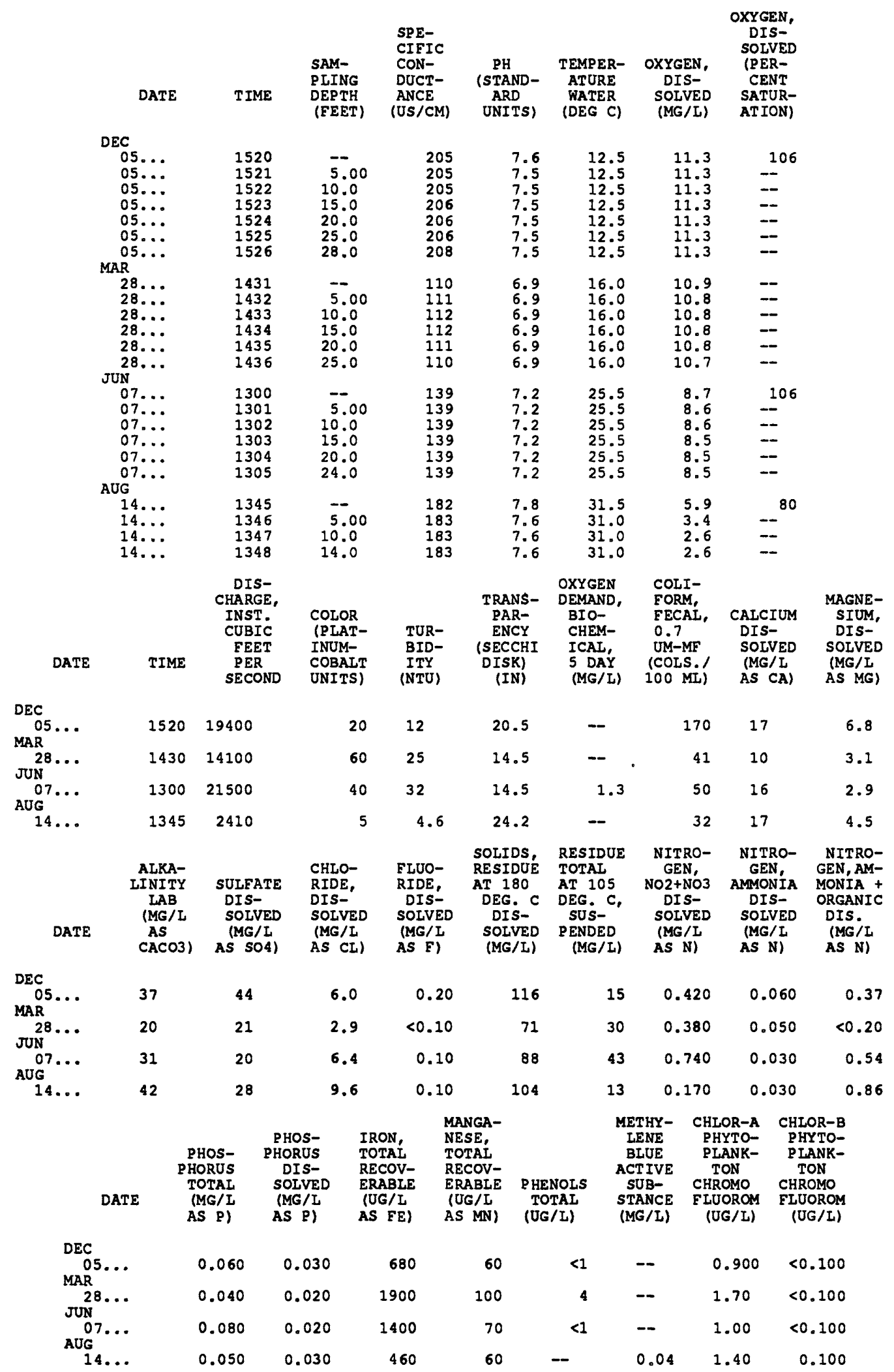


SURFACE-WATER SITES--Continued

02469525 TOMBIGBEE RIVER NEAR NANAFALIA, AL

\begin{tabular}{|c|c|c|c|c|}
\hline DATE & TIME & $\begin{array}{l}\text { SAMP LE } \\
\text { LOCAT. } \\
\text { X-SECT. } \\
\text { LOOKING } \\
\text { UP STRM. } \\
\text { (8 FROM } \\
\text { R BANK) }\end{array}$ & $\begin{array}{c}\text { DIS- } \\
\text { CHARGE, } \\
\text { INST. } \\
\text { CUBIC } \\
\text { FEET } \\
\text { PER } \\
\text { SECOND }\end{array}$ & $\begin{array}{l}\text { SEDI- } \\
\text { MENT, } \\
\text { SUS- } \\
\text { PENDED } \\
\text { (MG / L) }\end{array}$ \\
\hline \multicolumn{5}{|l|}{ NOV } \\
\hline $\begin{array}{r}07 \ldots \\
07 \ldots \\
07 \ldots \\
\text { DEC }\end{array}$ & $\begin{array}{l}1030 \\
1035 \\
1040\end{array}$ & $\begin{array}{l}25.0 \\
50.0 \\
75.0\end{array}$ & $\begin{array}{l}E 20000 \\
E 20000 \\
E 20000\end{array}$ & $\begin{array}{l}27 \\
32 \\
30\end{array}$ \\
\hline $\begin{array}{r}21 \ldots \\
21 \ldots \\
21 \ldots \\
\end{array}$ & $\begin{array}{l}1010 \\
1015 \\
1020\end{array}$ & $\begin{array}{l}25.0 \\
50.0 \\
75.0\end{array}$ & $\begin{array}{l}E 24000 \\
E 24000 \\
E 24000\end{array}$ & $\begin{array}{l}63 \\
83 \\
72\end{array}$ \\
\hline $\begin{array}{r}02 \ldots \\
02 \ldots \\
02 \ldots \\
\text { ARR }\end{array}$ & $\begin{array}{l}1225 \\
1230 \\
1235\end{array}$ & $\begin{array}{l}25.0 \\
50.0 \\
75.0\end{array}$ & $\begin{array}{l}E 80000 \\
E 80000 \\
E 80000\end{array}$ & $\begin{array}{r}91 \\
167 \\
172\end{array}$ \\
\hline $\begin{array}{r}26 \ldots \\
26 \ldots \\
26 \ldots \\
\text { JUN }\end{array}$ & $\begin{array}{l}1340 \\
1345 \\
1350\end{array}$ & $\begin{array}{l}25.0 \\
50.0 \\
75.0\end{array}$ & $\begin{array}{l}\text { E15000 } \\
\text { E15000 } \\
\text { E15000 }\end{array}$ & $\begin{array}{l}32 \\
30 \\
31\end{array}$ \\
\hline $\begin{array}{l}07 \ldots \\
07 \ldots \\
07 \ldots \\
\text { JUL }\end{array}$ & $\begin{array}{l}0800 \\
0755 \\
0750\end{array}$ & $\begin{array}{l}25.0 \\
50.0 \\
75.0\end{array}$ & $\begin{array}{l}E 25000 \\
E 25000 \\
E 25000\end{array}$ & $\begin{array}{l}60 \\
71 \\
84\end{array}$ \\
\hline $\begin{array}{l}18 \ldots \\
18 \ldots \\
18 \ldots\end{array}$ & $\begin{array}{l}1650 \\
1655 \\
1700\end{array}$ & $\begin{array}{l}25.0 \\
50.0 \\
75.0\end{array}$ & $\begin{array}{l}\text { E7000 } \\
\text { E7000 } \\
\text { E7000 }\end{array}$ & $\begin{array}{l}16 \\
15 \\
17\end{array}$ \\
\hline
\end{tabular}

02469762 TOMBIGBEE RIVER BELOW COFFEEVILLE LOCK AND DAM, AL

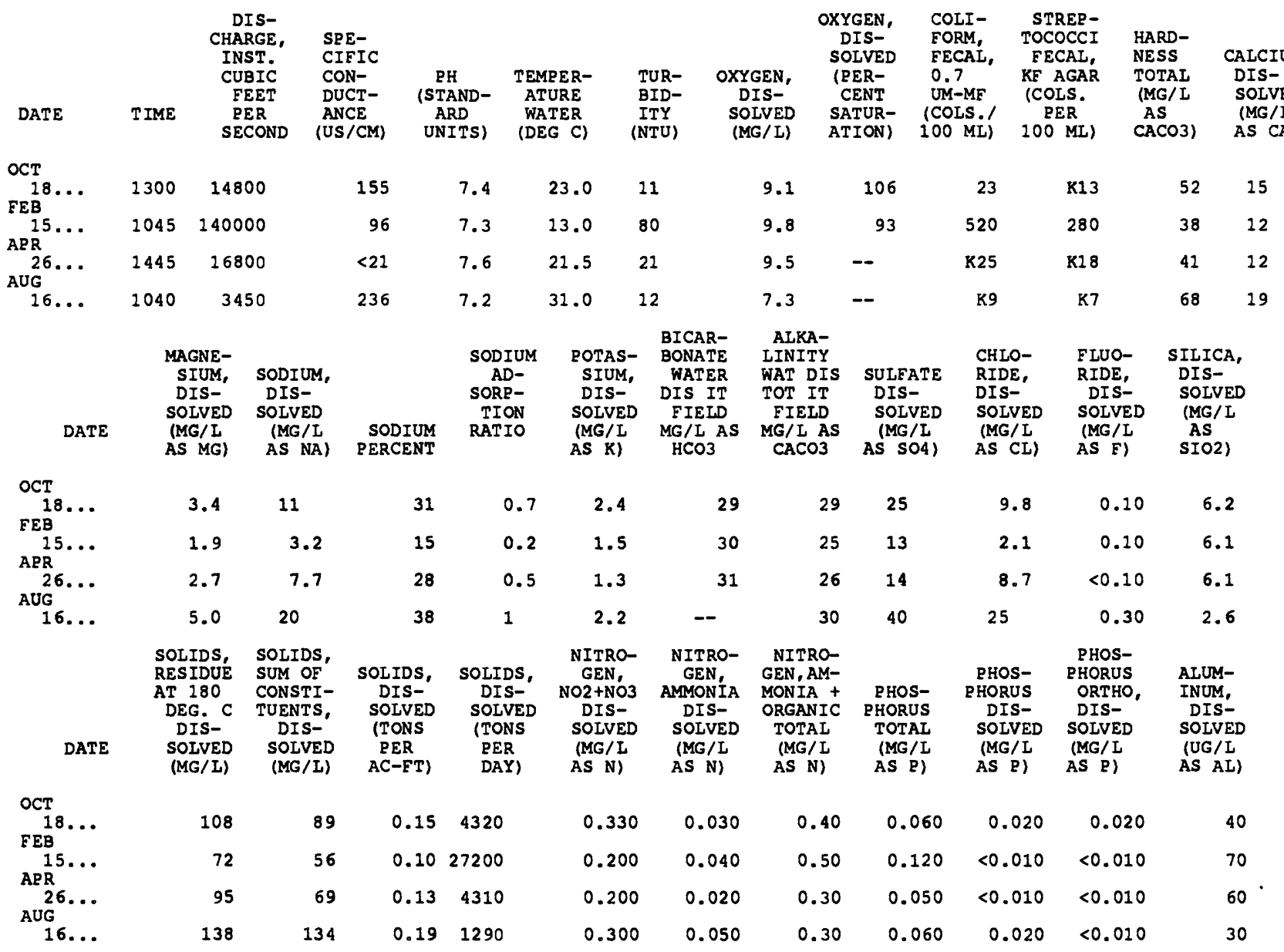


SURFACE-WATER SITES--Cont1nued

02469762 TOMBIGBEE RIVER BELOW COFFEEVIILE LOCK AND DAM, AL--Continued

$\begin{array}{cclc}\text { ARSENIC } & \text { BARIUM, } & \begin{array}{l}\text { BERYL- } \\ \text { LIUM, }\end{array} & \text { CADMIUM } \\ \text { DIS- } & \text { DIS- } & \text { DIS- } & \text { DIS- } \\ \text { SOLVED } & \text { SOLVED } & \text { SOLVED } & \text { SOLVED } \\ \text { (UG/L } & \text { (UG/L } & \text { (UG/L } & \text { (UG/L } \\ \text { AS AS) } & \text { AS BA) } & \text { AS BE) } & \text { AS CD) }\end{array}$

$\begin{array}{lcc}\text { CHRO- } & & \\ \text { MIUM, } & \text { COBALT, } & \text { COPPER, } \\ \text { DIS- } & \text { DIS- } & \text { DIS- } \\ \text { SOLVED } & \text { SOIVED } & \text { SOLVED } \\ \text { (UG/L } & \text { (UG/L } & \text { (UG/L } \\ \text { AS CR) } & \text { AS CO) } & \text { AS CU) }\end{array}$

\begin{tabular}{|c|c|c|}
\hline $\begin{array}{l}\text { IRON, } \\
\text { DIS- } \\
\text { SOLVED } \\
\text { (UG/L } \\
\text { AS FE) }\end{array}$ & $\begin{array}{l}\text { IEAD, } \\
\text { DIS- } \\
\text { SOLVED } \\
\text { (UG/I } \\
\text { AS PB) }\end{array}$ & $\begin{array}{l}\text { IITHIUM } \\
\text { DIS- } \\
\text { SOLVED } \\
\text { (UG/L } \\
\text { AS LI) }\end{array}$ \\
\hline
\end{tabular}

OCT
$18 \ldots$
FEB
$15 \ldots$
APR
$26 \ldots$
AUG
$16 \ldots$

\begin{tabular}{|c|c|c|c|c|}
\hline$<1$ & 28 & $<0.5$ & $<1.0$ & 2 \\
\hline$<1$ & 19 & $<0.5$ & 1.0 & $<5$ \\
\hline$<1$ & 29 & $<0.5$ & $<1.0$ & $<1$ \\
\hline$<1$ & 35 & $<0.5$ & $<1.0$ & $<1$ \\
\hline 0 & $\begin{array}{l}\text { MOLYB- } \\
\text { DENUM, } \\
\text { DIS- } \\
\text { SOLVED } \\
\text { (UG/L } \\
\text { AS MO) }\end{array}$ & $\begin{array}{l}\text { NICKEL, } \\
\text { DIS- } \\
\text { SOLVED } \\
\text { (UG/L } \\
\text { AS NI) }\end{array}$ & $\begin{array}{l}\text { SELE- } \\
\text { NIUM, } \\
\text { DIS- } \\
\text { SOLVED } \\
\text { (UG/L } \\
\text { AS SE) }\end{array}$ & $\begin{array}{c}\text { SILVER, } \\
\text { DIS- } \\
\text { SOLVED } \\
\text { (UG/L } \\
\text { AS AG) }\end{array}$ \\
\hline
\end{tabular}

$\begin{array}{rr}<3 & 2 \\ <3 & <10 \\ <3 & 2 \\ <3 & 2 \\ & \\ \text { VRON- } & \begin{array}{c}\text { VANA- } \\ \text { DIUM, } \\ \text { DIS- } \\ \text { DIS- } \\ \text { SOLVED } \\ \text { (UG/L }\end{array} \\ \text { UG /L } & \text { AS V) }\end{array}$

130
96
250
110

$\begin{array}{rrr}<1 & <4 & 7 \\ <10 & <4 & 15 \\ 2 & <4 & 4 \\ 1 & 5 & 5\end{array}$

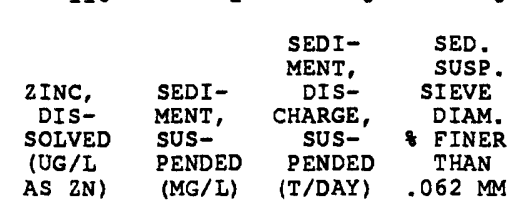

OCT
$18 \ldots$
FEB
$15 \ldots$
ARR
$26 \ldots$
AUG
$16 \ldots$

\begin{tabular}{|c|c|c|c|c|c|c|c|c|c|c|}
\hline $18 \ldots$ & $<0.1$ & $<10$ & 1 & $<1$ & $<1.0$ & 93 & $<6$ & 110 & 599 & 9 \\
\hline & $<0.1$ & $<10$ & $<10$ & $<1$ & $<1.0$ & 68 & $<6$ & 22 & 25194900 & 6 \\
\hline $26 \ldots$ & 0.2 & $<10$ & 1 & $<1$ & $<1.0$ & 96 & $<6$ & $<3$ & 635 & 9 \\
\hline $16 \ldots$ & $<0.1$ & $<10$ & 1 & $<1$ & $<1.0$ & 110 & $<6$ & 78 & 121 & 9 \\
\hline & DATE & $\begin{array}{l}\text { GROSS } \\
\text { ALPHA, } \\
\text { DIS- } \\
\text { SOLVED } \\
\text { (UG/L } \\
\text { AS } \\
\text { U-NAT) }\end{array}$ & $\begin{array}{l}\text { GROSS } \\
\text { ALP HA, } \\
\text { SUSP. } \\
\text { TOTAL } \\
\text { (UG/I } \\
\text { AS } \\
\text { U-NAT) }\end{array}$ & $\begin{array}{c}\text { GROSS } \\
\text { BETA, } \\
\text { DIS- } \\
\text { SOIVED } \\
\text { (PCI/L } \\
\text { AS } \\
\text { CS-137) }\end{array}$ & $\begin{array}{c}\text { GROSS } \\
\text { BETA, } \\
\text { SUSP } \\
\text { TOTAI } \\
\text { (PCI/L } \\
\text { AS } \\
\text { CS-137) }\end{array}$ & $\begin{array}{l}\text { GROSS } \\
\text { BETA, } \\
\text { DIS- } \\
\text { SOLVED } \\
\text { (PCI/L } \\
\text { AS SR/ } \\
\text { YT-90) }\end{array}$ & $\begin{array}{l}\text { GROSS } \\
\text { BETA, } \\
\text { SUSP. } \\
\text { TOTAI } \\
\text { (PCI/L } \\
\text { AS SR/ } \\
\text { YT-9O) }\end{array}$ & $\begin{array}{c}\text { RADIUM } \\
226, \\
\text { DIS- } \\
\text { SOLVED, } \\
\text { RADON } \\
\text { METHOD } \\
(\mathrm{PCI} / \mathrm{L})\end{array}$ & $\begin{array}{l}\text { URANIUM } \\
\text { NATURAL } \\
\text { DIS- } \\
\text { SOLVED } \\
\text { (UG/L } \\
\text { AS U) }\end{array}$ & \\
\hline & \multirow{2}{*}{$\begin{array}{c}\text { FEB } \\
15 \ldots \\
\text { AUG } \\
16 \ldots\end{array}$} & 0.6 & 0.5 & 2.1 & 0.7 & 1.8 & 0.6 & 0.07 & \multirow[t]{2}{*}{$<0.01$} & \\
\hline & & $<0.4$ & 0.7 & 2.5 & 0.7 & 2.0 & 0.6 & $-\infty$ & & \\
\hline
\end{tabular}

$\begin{array}{lll}(U G / L & (U G / I \\ \text { AG) } & \text { AS SR) }\end{array}$

AS $2 N$ )

MG/L)

(T/DAY) $\quad .062 \mathrm{MM}$

SPECIFIC CONDUCTANCE, MICROSIEMENS PER CENTIMETER AT 25 DEG. C, WATER YEAR OCTOBER 1989 TO SEPTEMBER 1990

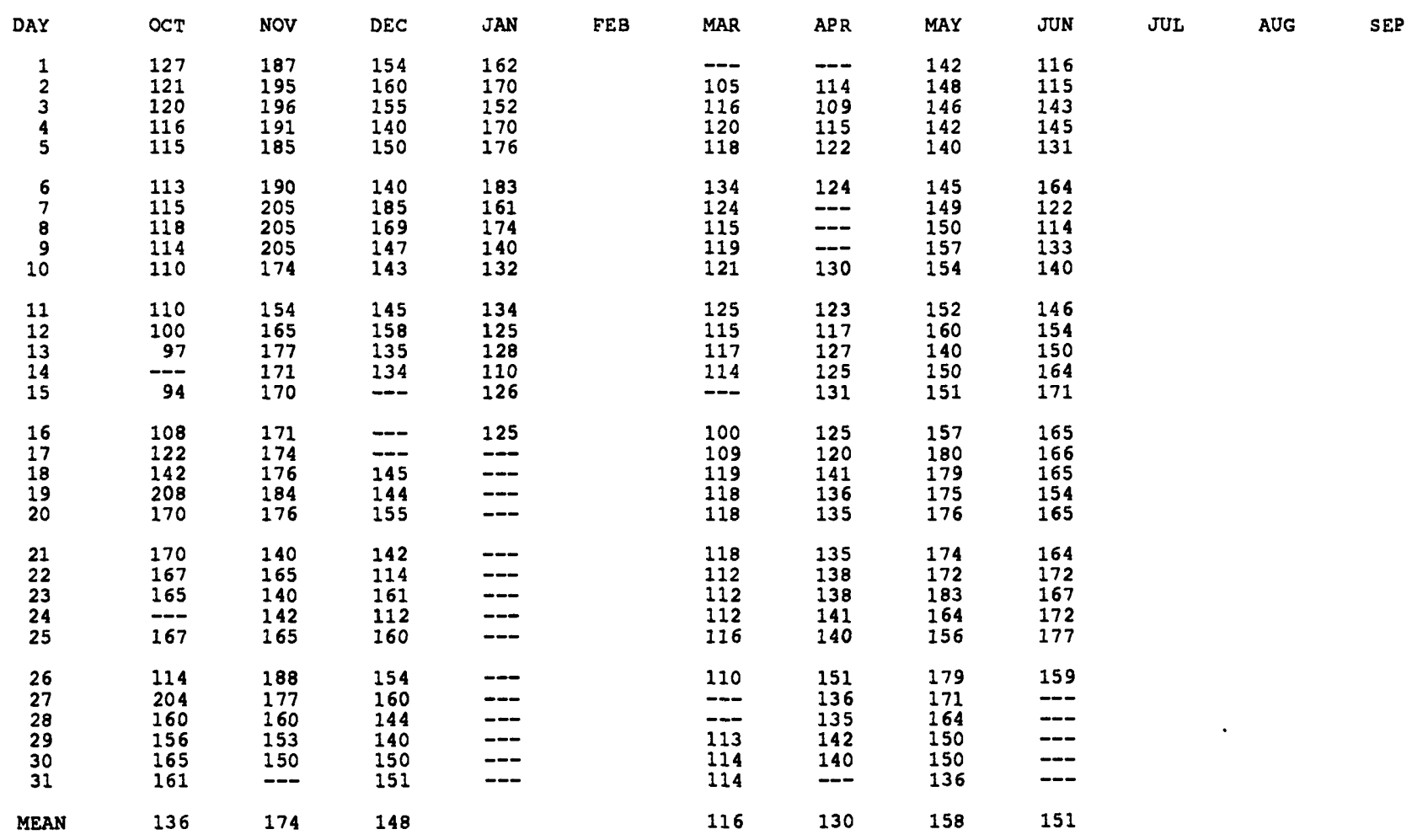


SURFACE-WATER SITES--Cont1nued

02469762 TOMBIGBEE RIVER BELOW COFFEEVILLE LOCK AND DAM, AL--Continued TEMPERATURE, WATER (DEG. C), WATER YEAR OCTOBER 1989 TO SEPTEMBER 1990

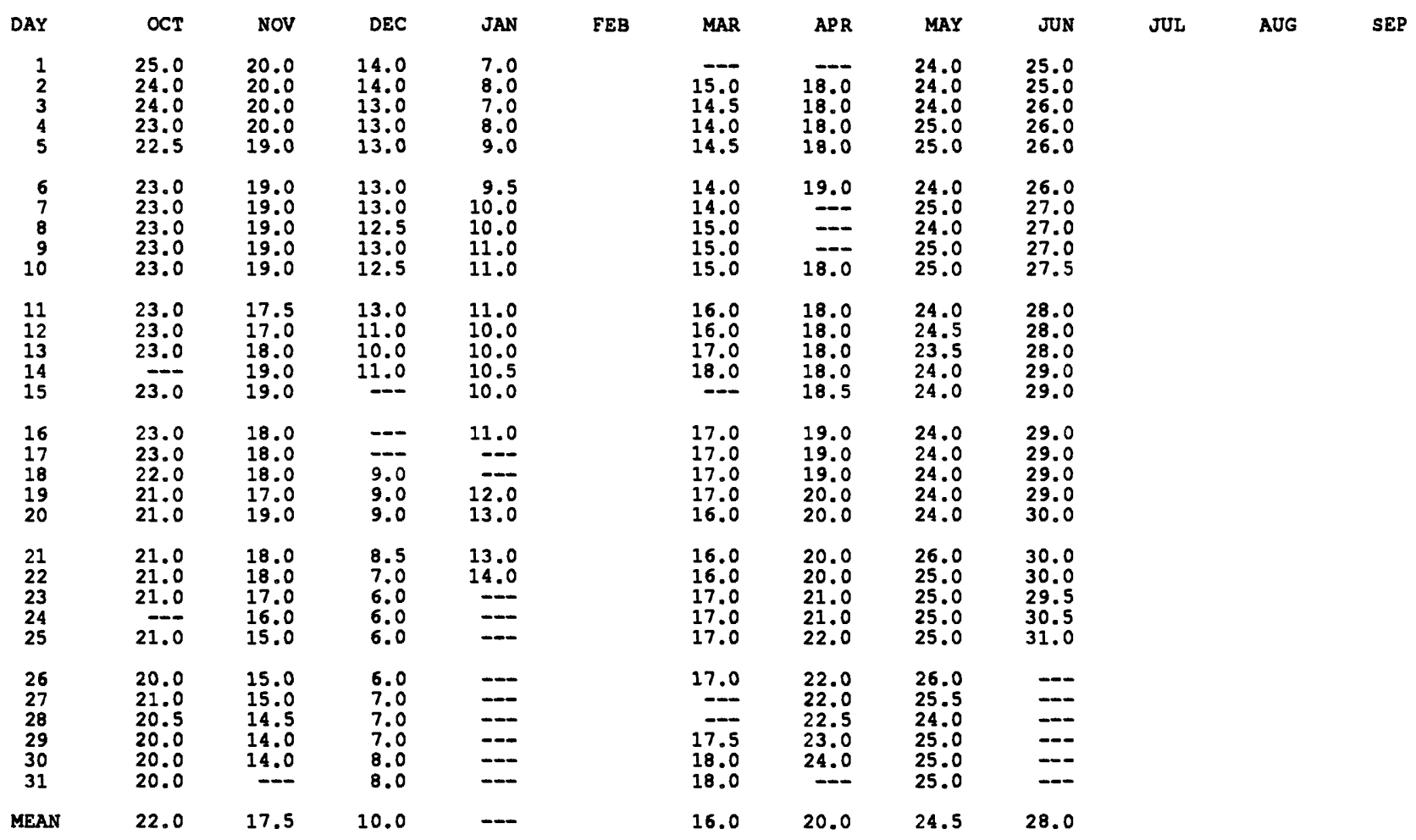

